\title{
Vehicle Inertia Impact on Fuel Consumption of Conventional and Hybrid Electric Vehicles Using Acceleration and Coast Driving Strategy
}

\section{Jeongwoo Lee}

\author{
Dissertation submitted to the faculty of the \\ Virginia Polytechnic Institute and State University \\ in partial fulfillment of the requirements of the degree of
}

\section{DOCTOR OF PHILLOSOPHY \\ in}

Mechanical Engineering

\author{
Douglas J. Nelson, Committee Chair \\ Michael W. Ellis \\ Dennis W. Hong \\ Kevin Kochersberger \\ Jason Lai
}

September 4, 2009

Blacksburg, Virginia, USA

Key words: Acceleration, Coasting, Kinetic Energy, Vehicle Inertia, Hybrid Electric Vehicle,

Control Strategy, Fuel Consumption

(C) Copyright 2009 by Jeongwoo Lee

All Rights Reserved 


\title{
Vehicle Inertia Impact on Fuel Consumption of Conventional and Hybrid Electric Vehicles Using Acceleration and Coast Driving Strategy
}

\author{
Jeongwoo Lee
}

\begin{abstract}
In the past few years, the price of petroleum based fuels, especially vehicle fuels such as gasoline and diesel, has been increasing at a significant rate. Consequently, there is much more consumer interest related to reducing fuel consumption for conventional vehicles and hybrid electric vehicles (HEVs) than in the past.

The goal of many competitions and challenges held in North America and Europe is to achieve extremely low fuel consumption. A possible strategy to reduce fuel consumption is to use the vehicle's fuel converter such as an engine to accelerate the vehicle to a high speed and coast to a lower speed with the engine off. This method will reduce fuel flow to zero during the coast phase. Also, the vehicle uses higher power engine load to accelerate to the upper vehicle speed in a limited time, thus increasing the engine brake thermal efficiency. This strategy is known as "pulse and glide" or "burn and coast" in some references.

In this study, the "pulse and glide" (PnG) method is first applied to a conventional vehicle to quantify the fuel consumption benefits when compared to steady speed conditions over the same distance. After that, an HEV is used as well to investigate if a hybrid system can further reduce fuel consumption with the proposed strategy. Note that the HEV used in this study has the advantage that the engine can be automatically shut off below a certain speed ( $\sim 40 \mathrm{mph}$ ) at low loads, however a driver must shut off the engine manually in a conventional vehicle to apply this driving strategy.
\end{abstract}


In this document, three preliminary results of the PnG driving strategy are presented; (1) improved fuel economy for a conventional vehicle from a simple spread sheet model, (2) improved fuel economy for an HEV from a dynamic vehicle simulation model (the Powertrain Analysis Toolkit (PSAT)) and (3) improved fuel economy for an HEV from vehicle testing at Argonne National Laboratory (ANL), all compared to steady speed conditions. The preliminary results show that the impact of engine load and kinetic energy stored in vehicle inertia is significant for fuel consumption using a PnG driving strategy compared to steady speed driving at the same average speed case. Especially, fuel economy can be improved at low speed range and higher acceleration because the aerodynamic drag force is smaller at low speed and the engine is running in a more efficient region for a short period of time respectively. In the last section, proposed directions of research are addressed based on the preliminary results. 


\section{Acknowledgements}

This research is funded by the Graduate Automotive Technology Education (GATE) program of Department of Energy (DoE).

First of all, I would like to thank of all my committee members, Drs. Douglas Nelson, Michael Ellis, Dennis Hong, Kevin Kochersberger and Jason Lai for giving valuable input and guidance to complete this dissertation. A special thanks to Dr. Douglas Nelson for his continuous guidance, advice, patience and kindness as my advisor since master's program and for giving me an opportunity to achieve my goals at Virginia Tech. I could see and learn his constant passion for vehicle research for the last 6 years. I also want to thank Henning Lohse-Busch for testing vehicles at Argonne National Laboratory (ANL) and sharing his valuable opinions.

Last but not least, I want to thank my parents, family and friends, especially, my wife, Soyoun, and my daughter, Gyueun. Without their endless support and encouragement, I could not complete my Ph.D. program at Virginia Tech. I always love you all. 


\section{Table of Contents}

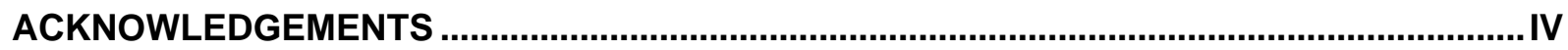

TABLE OF CONTENTS

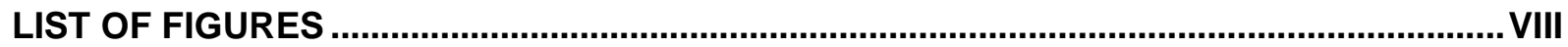

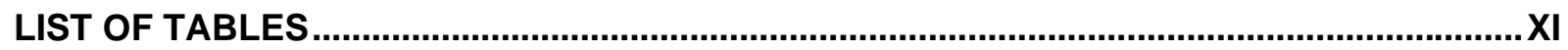

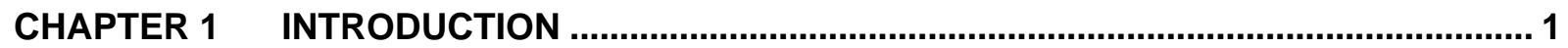

$1.1 \quad$ Increase of Petroleum Based Fuel Use................................................................................ 1

1.2 Introduction of Eco-Driving Strategies ........................................................................... 1

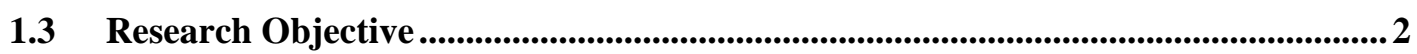

CHAPTER 2 LITERATURE REVIEW

2.1 Overview of Pulse and Glide (PnG) Driving Strategy .................................................... 4

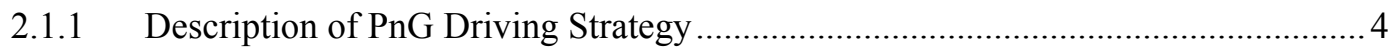

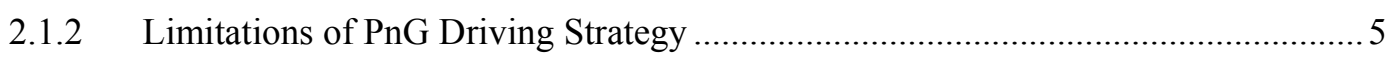

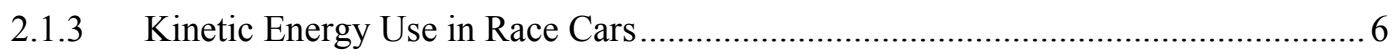

2.2 Impact Factors on Fuel Consumption of Vehicles............................................................. 7

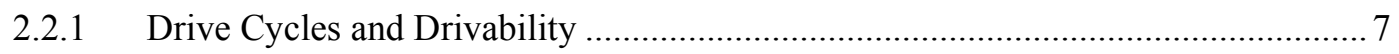

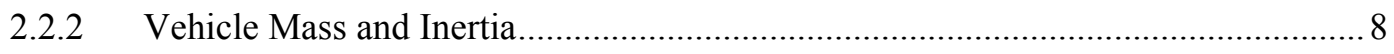

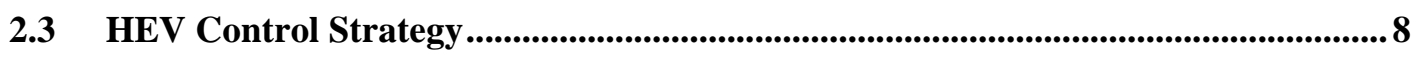

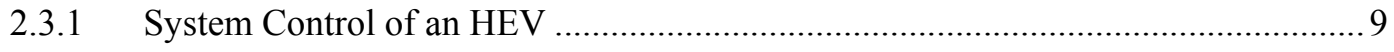

2.3.2 Control Strategy Based on Power Loss Calculation ……......................................... 10

2.3.3 Control Strategy Using Driving Pattern Recognition ............................................ 10

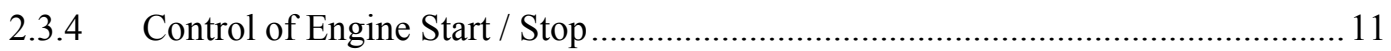

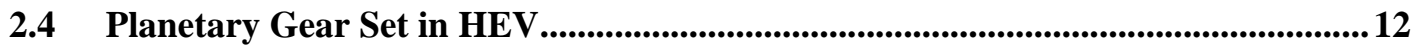

2.5 Concerns for Testing Hybrid Electric Vehicles (HEVs)...............................................14

2.6 Research Contribution .......................................................................................... 15

CHAPTER 3 SIMPLE VEHICLE MODELING AND PARAMETRIC STUDY FOR A CONVENTIONAL VEHICLE ..................................................................... 16

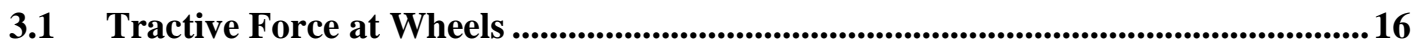

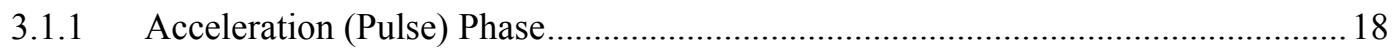

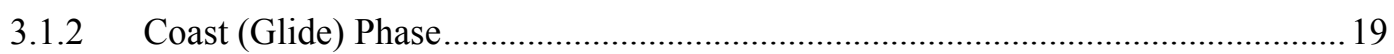

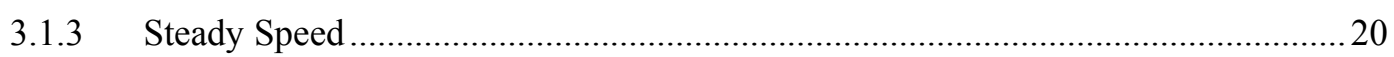


3.2 Simple Vehicle Modeling for a Conventional Vehicle ................................................ 20

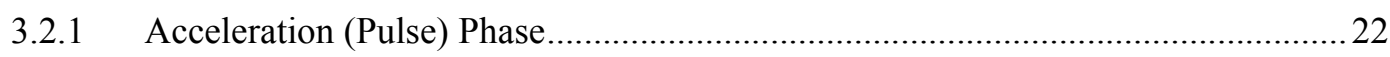

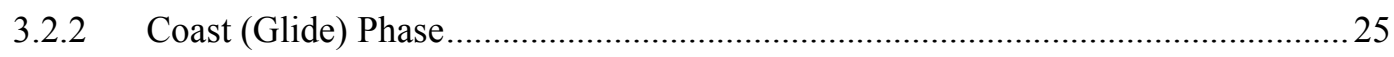

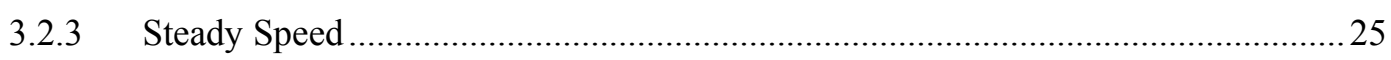

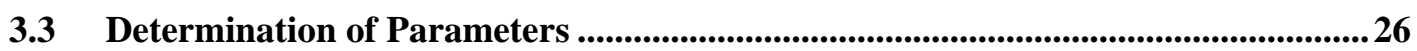

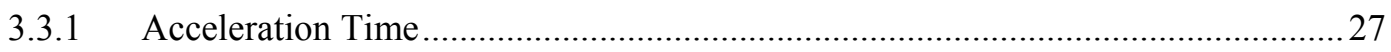

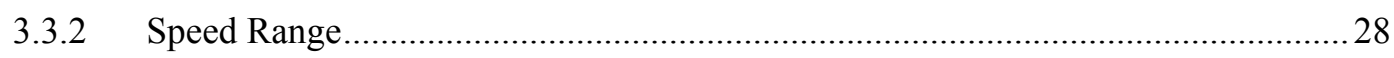

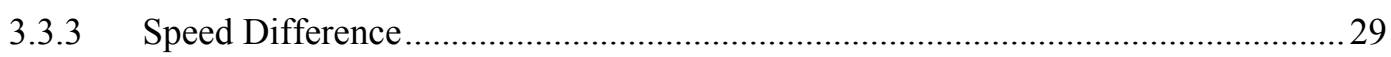

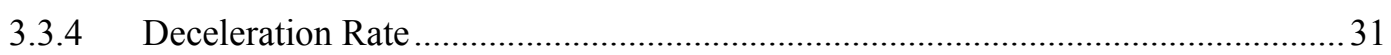

3.4 Efficiency of Kinetic Energy Storage and Net Benefit of PnG ..................................34

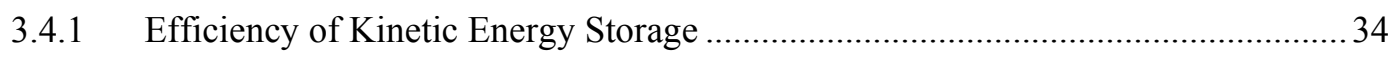

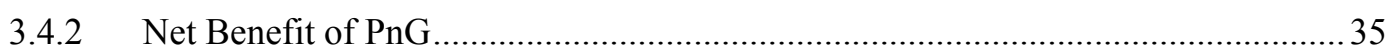

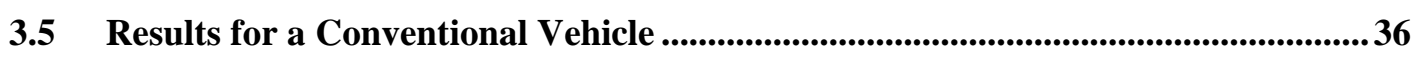

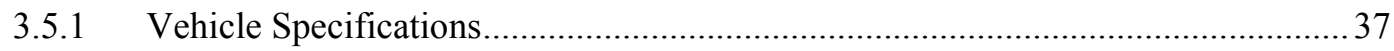

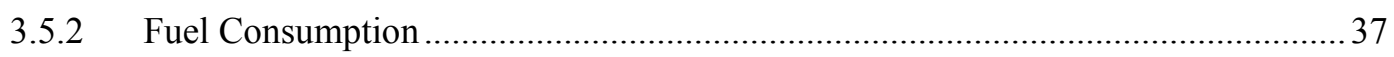

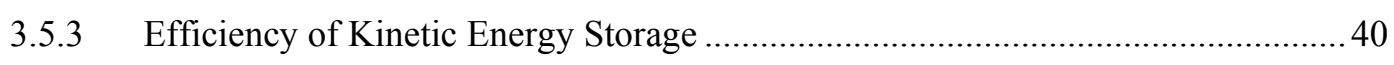

CHAPTER 4 IN-DEPTH VEHICLE SIMULATION FOR CONVENTIONAL AND HYBRID ELECTRIC VEHICLES USING POWERTRAIN SYSTEM ANALYSIS TOOLKIT (PSAT) ...................................................................................... 42

4.1 Simulation of PnG and Steady Speed using PSAT ........................................................ 42

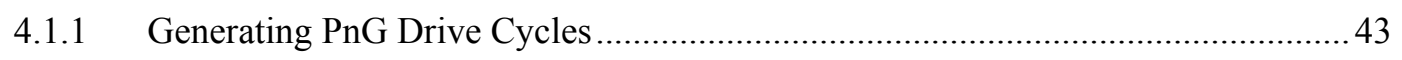

4.1.2 Method of Data Extraction from Simulation Results............................................. 44

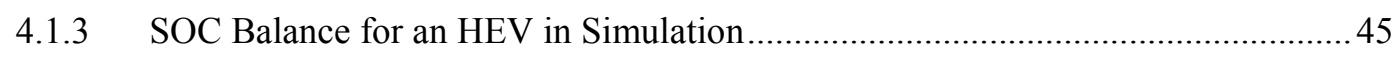

4.1.4 Selection of PnG Cases for In-Depth Simulation................................................. 46

4.2 Conventional Vehicle: 2007 Ford Focus ..................................................................4

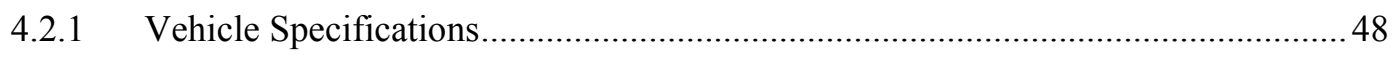

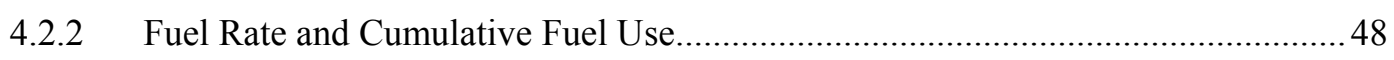

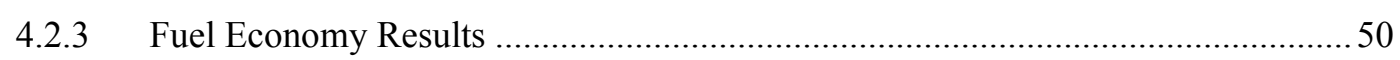

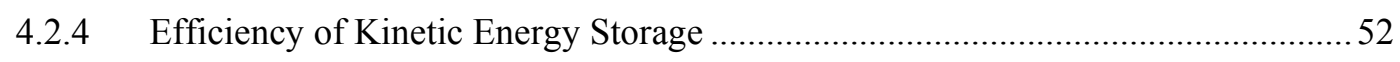

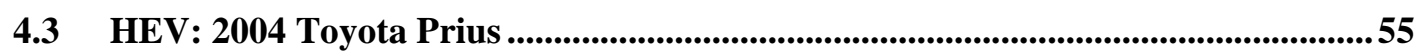

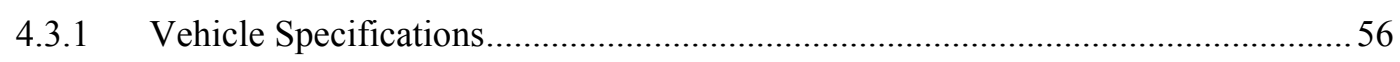

4.3.2 Fuel Rate, SOC of Battery and Cumulative Fuel Use..............................................56

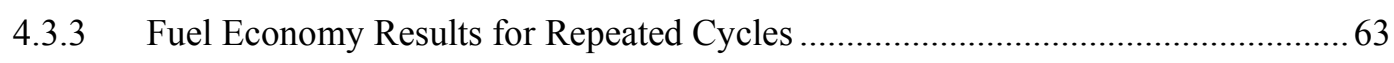

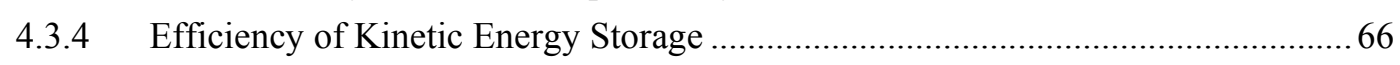

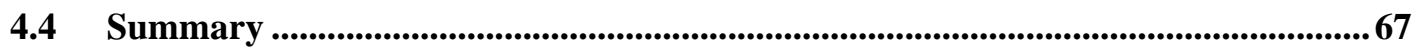


CHAPTER 5 EXPERIMENTAL SETUP AND VEHICLE TESTING FOR AN HEV ...............69

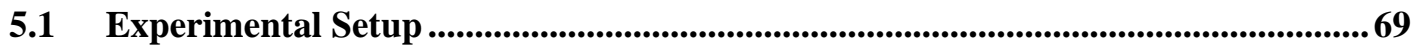

5.2 Experimental Design: Selected Cases ........................................................................ 72

CHAPTER 6 EXPERIMENTAL RESULTS AND DISCUSSION ......................................... 76

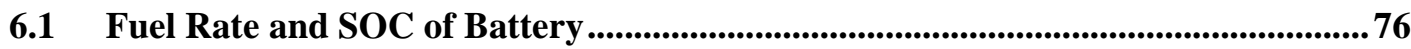

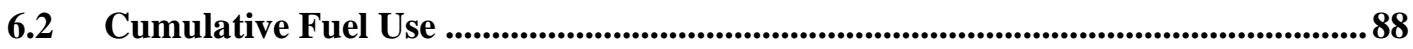

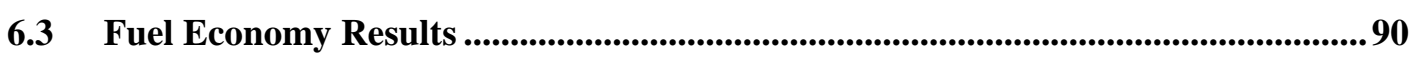

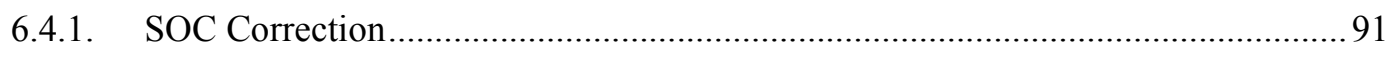

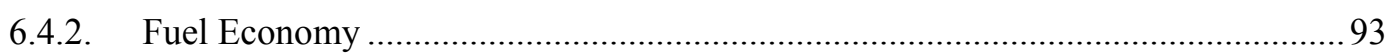

6.4.3. Comparison with Simulation Results ....................................................................96

6.5. Efficiency of Kinetic Energy Storage..................................................................................99

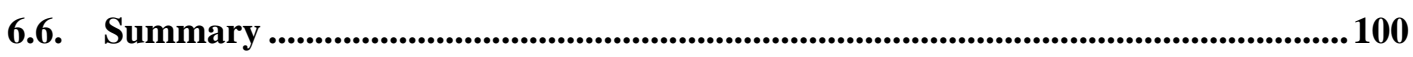

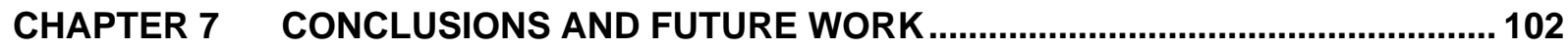

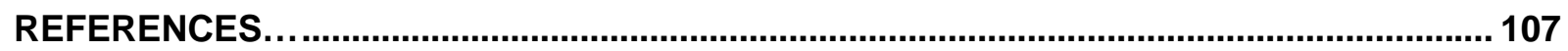

APPENDIX A SPEED, TORQUE AND POWER OF A PLANETARY GEAR SET................ 112

APPENDIX B RESULT DATA FROM SIMULATIONS AND VEHICLE TESTING............... 115

APPENDIXC SOC CORRECTION FOR FUEL ECONOMY OF HEVS USING LINEAR

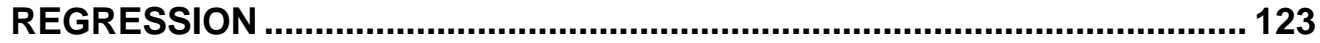




\section{List of Figures}

Figure 2.1 PnG with engine on (a) and engine off (b) during coasting .................................................. 5

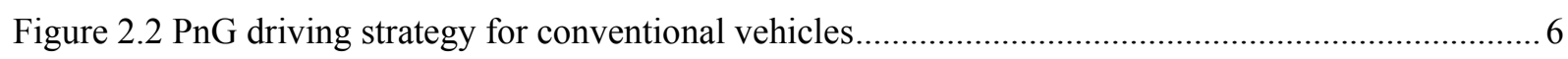

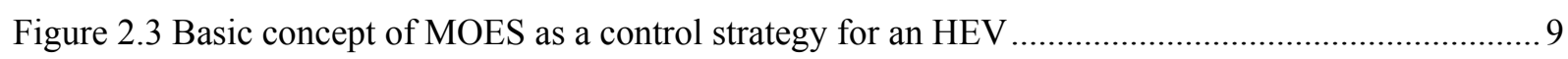

Figure 2.4 Schematic of a HEV control strategy using driving pattern recognition (DPR) method........... 11

Figure 2.5 Driveline with the power split device (PSD) in 2004 Toyota Prius ......................................... 13

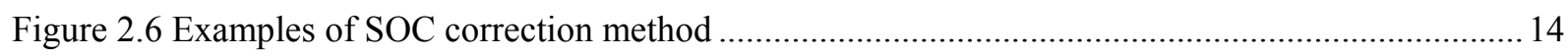

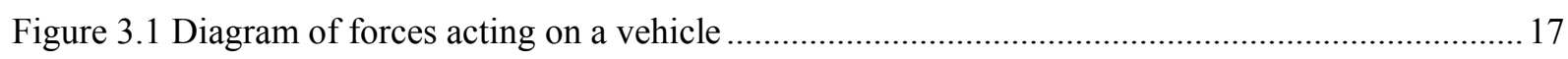

Figure 3.2 Schematic of the simple vehicle modeling for a conventional vehicle...................................... 21

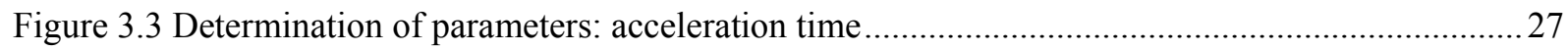

Figure 3.4 Engine operating region for different acceleration rates, fixed gear condition ........................28

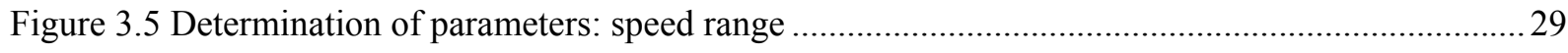

Figure 3.6 Engine operating region for different speed ranges, fixed gear condition...............................2

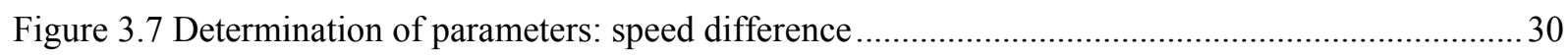

Figure 3.8 Engine operating for two speed differences, fixed gear condition .......................................... 30

Figure 3.9 Vehicle speed comparison between coast down and engine compression braking for a

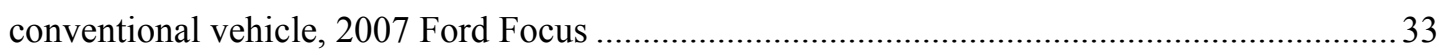

Figure 3.10 Deceleration rate comparison between coast down and engine compression braking for a

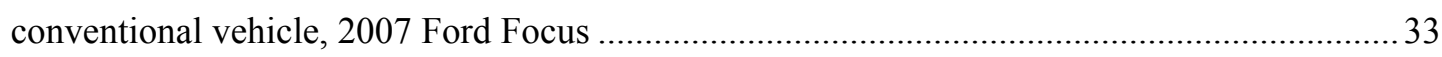

Figure $3.11 \mathrm{PnG}$ and steady speed cases with two separated intervals .................................................... 35

Figure 3.12 Contour map of fuel economy results of PnG from the simple vehicle model along with different acceleration times and speed ranges, 2007 Ford Focus............................................... 38

Figure 3.13 Fuel economy results from simple vehicle model in different speed ranges and acceleration times, 2007 Ford Focus

Figure 3.14 Fuel economy results from simple vehicle model between $25 \mathrm{mph}$ and $45 \mathrm{mph}$ in different acceleration times, 2007 Ford Focus 40

Figure 3.15 Efficiency of KE storage during acceleration in PnG, 2007 Ford Focus................................. 41

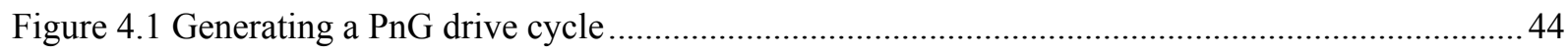

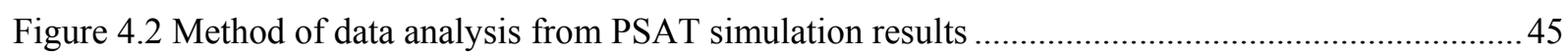

Figure 4.3 Example of data extraction for SOC balance from an HEV simulation .....................................46

Figure 4.4 Selected PnG cases for in-depth simulation using PSAT for the conventional vehicle and the HEV 
Figure 4.5 Fuel rate comparison between steady speed at $25 \mathrm{mph}$ and PnG in $20-30 \mathrm{mph}$ with 10 seconds of acceleration in gear 3, 2007 Ford Focus

Figure 4.6 Fuel rate comparison between steady speed at $25 \mathrm{mph}$ and PnG in $20-30 \mathrm{mph}$ with 10 seconds of acceleration in gear 3, 2007 Ford Focus ................................................................5 50

Figure 4.7 Fuel economy comparison for different acceleration time, 2007 Ford Focus .............................52

Figure 4.8 Efficiency of KE storage for different acceleration time from PSAT, 2007 Ford Focus .......... 53

Figure 4.9 Engine efficiency of PnG during acceleration in 20 - $30 \mathrm{mph}$ from PSAT, 2007 Ford Focus .53

Figure 4.10 Engine efficiency of PnG from PSAT and simple vehicle model, 2007 Ford Focus .54

Figure 4.11 Fuel rate comparison between steady speed at $25 \mathrm{mph}$ and PnG in 20 - $30 \mathrm{mph}$ with 10 seconds of acceleration, 2004 Toyota Prius

Figure 4.12 SOC and battery output power of PnG in 20 - $30 \mathrm{mph}$ with 10 seconds of acceleration, 2004

Toyota Prius .58

Figure 4.13 Fuel rate, SOC and battery output power of PnG in $20-30 \mathrm{mph}$ with 5 seconds of acceleration, 2004 Toyota Prius

Figure 4.14 Fuel rate, SOC and battery output power of PnG in $20-30$ mph with 30 seconds of acceleration, 2004 Toyota Prius.

Figure 4.15 Cumulative fuel use comparison between steady speed at $25 \mathrm{mph}$ and PnG in $20-30 \mathrm{mph}$

with 10 seconds of acceleration in gear 3, 2004 Toyota Prius

Figure 4.16 Fuel economy comparison for different acceleration time, 2004 Toyota Prius.........................64

Figure 4.17 Fuel use comparison during acceleration and coasting, 2004 Toyota Prius ..............................65

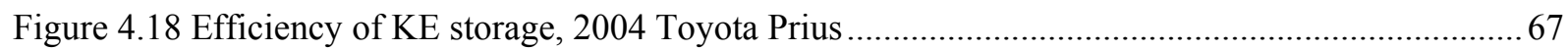

Figure 5.1 Test vehicle, 2004 Toyota Prius, on a dynamometer in ANL ……........................................ 70

Figure 5.2 Schematic of vehicle testing facility in ANL ................................................................... 71

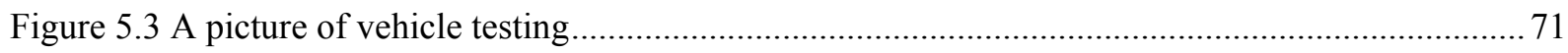

Figure 5.4 Different deceleration rates for vehicle testing, 20 - $30 \mathrm{mph}$ of PnG driving .......................... 74

Figure 6.1 Fuel rate and SOC of battery in the steady speed driving from vehicle testing, 2004 Toyota

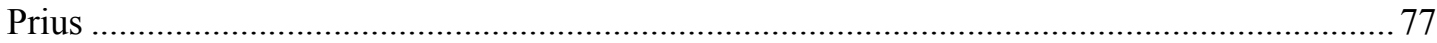

Figure 6.2 Engine efficiency and engine power for optimized engine operating line of 2004 Toyota Prius

Figure 6.3 Fuel rate and SOC of battery in the PnG driving in true coast down with gear in N position from vehicle testing, 2004 Toyota Prius

Figure 6.4 Engine fuel rate and battery power of the PnG driving in $30-40 \mathrm{mph}$ from vehicle testing (test

5), 2004 Toyota Prius 82 
Figure 6.5 Output power in planetary gear of the PnG driving in $30-40 \mathrm{mph}$ from vehicle testing (test 5), 2004 Toyota Prius

Figure 6.6 Fuel rate and SOC of battery in the PnG driving with the same drive cycle as the PnG with true coast down and gear in D position from vehicle testing, 2004 Toyota Prius

Figure 6.7 Fuel rate and SOC of battery in the PnG driving with no driver's demand and gear in D position from vehicle testing, 2004 Toyota Prius

Figure 6.8 Fuel rate and SOC of battery in PnG driving with the same drive cycle as the PnG with slower deceleration and gear in D position from vehicle testing, 2004 Toyota Prius

Figure 6.9 Cumulative fuel use comparison for PnG in $20-30 \mathrm{mph}$ and steady speed at $25 \mathrm{mph}, 2004$

Toyota Prius

Figure 6.10 Cumulative fuel use comparison for PnG in 30-40 mph and steady speed at $35 \mathrm{mph}, 2004$

Toyota Prius

Figure 6.11 Cumulative fuel use comparison for PnG in $40-50 \mathrm{mph}$ and steady speed at $45 \mathrm{mph}, 2004$

Toyota Prius

Figure 6.12 Example of SOC correction for fuel economy of PnG (test 4), 2004 Toyota Prius

Figure 6.13 SOC corrected fuel economy results for PnG in $20-30 \mathrm{mph}$ and steady speed at $25 \mathrm{mph}$ from vehicle testing, 2004 Toyota Prius

Figure 6.14 SOC corrected fuel economy results for PnG in 30-40 mph and steady speed at $35 \mathrm{mph}$ from vehicle testing, 2004 Toyota Prius

Figure 6.15 SOC corrected fuel economy results for PnG in $40-50 \mathrm{mph}$ and steady speed at $45 \mathrm{mph}$ from vehicle testing, 2004 Toyota Prius

Figure 6.16 Fuel economy comparison between simulation and vehicle testing results for PnG with 10 seconds of acceleration and steady speed cases, 2004 Toyota Prius

Figure 6.17 Fuel use comparison of PnG during acceleration and coasting between simulation and vehicle testing (10 seconds of acceleration), 2004 Toyota Prius.

Figure 6.18 Efficiency of KE storage during acceleration for PnG (10 seconds of acceleration), 2004

Toyota Prius

Figure C.1 Example of SOC correction using linear regression from test 7.

Figure C.2 Example of SOC correction using linear regression from test 5 


\section{List of Tables}

Table 3.1 Vehicle specifications of 2007 Ford Focus used in the simple vehicle model

Table 3.2 Shortest acceleration time and fuel economy of PnG that the vehicle can achieve in the simple vehicle model (2007 Ford Focus)

Table 3.3 Selected cases to compare the fuel economy improvement of PnG and steady speed driving strategies in the simple vehicle model

Table 4.1 Vehicle specifications of 2007 Ford Focus used in PSAT.

Table 4.2 Fuel economy results for 2007 Ford Focus from PSAT simulation

Table 4.3 Selected cases to estimate the fuel economy improvement of PnG and steady speed driving strategies for 2004 Toyota Prius in PSAT

Table 4.4 Vehicle specifications of 2004 Toyota Prius used in PSAT ………........................................56

Table 4.5 Fuel economy results for 2004 Toyota Prius from PSAT simulation...........................................63

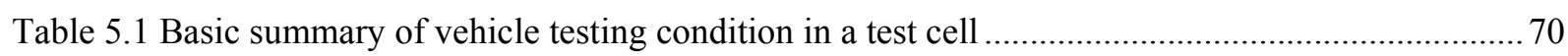

Table 5.2 Selected vehicle testing cases for the HEV, 2004 Toyota Prius ................................................ 73

Table 6.1 Fuel economy results for the HEV from vehicle testing, 2004 Toyota Prius .............................. 93

Table B.1 Results from the simple vehicle model for finding optimized fuel economy of PnG driving (2007 Ford Focus).

Table B.2 Results from the simple vehicle model for PnG in 20 - $30 \mathrm{mph}$ and steady speed at $25 \mathrm{mph}$ (2007 Ford Focus).

Table B.3 Results from the simple vehicle model for PnG in $30-40 \mathrm{mph}$ and steady speed at $35 \mathrm{mph}$ (2007 Ford Focus).

Table B.4 Results from the simple vehicle model for PnG in 40 - $50 \mathrm{mph}$ and steady speed sped at 45 mph (2007 Ford Focus)

Table B.5 Results from PSAT for PnG in 20 - $30 \mathrm{mph}$ and steady speed at $25 \mathrm{mph}$ (2007 Ford Focus) 117

Table B.6 Results from PSAT for PnG in $30-40 \mathrm{mph}$ and steady speed at $35 \mathrm{mph}$ (2007 Ford Focus) 118

Table B.7 Results from PSAT for PnG in $40-50 \mathrm{mph}$ and steady speed at $45 \mathrm{mph}$ (2007 Ford Focus) 118

Table B.8 Results from PSAT for PnG in 20 - $30 \mathrm{mph}$ and steady speed at $25 \mathrm{mph}$ (2004 Toyota Prius)

Table B.9 Results from PSAT for PnG in 30 - $40 \mathrm{mph}$ and steady speed at $35 \mathrm{mph}$ (2004 Toyota Prius)

Table B.10 Results from PSAT for PnG in 40 - $50 \mathrm{mph}$ and steady speed at $45 \mathrm{mph}$ (2004 Toyota Prius) 
Table B.11 Results from vehicle testing for PnG in $20-30 \mathrm{mph}$ and steady speed at $25 \mathrm{mph}$ (2004 Toyota Prius) 122

Table B.12 Results from vehicle testing for PnG in $30-40 \mathrm{mph}$ and steady speed at $35 \mathrm{mph}$ (2004 Toyota Prius)

Table B.13 Results from vehicle testing for PnG in $40-50 \mathrm{mph}$ and steady speed at $45 \mathrm{mph}$ (2004 Toyota Prius) 


\section{Chapter 1 Introduction}

\subsection{Increase of Petroleum Based Fuel Use}

The petroleum based fuel consumption has increased for the last few decades in the U.S. Especially, the consumption of fuels such as gasoline or diesel have increased because more vehicles have been produced and vehicle miles traveled (VMT) has significantly increased as well. The increase of petroleum based fuel consumption can increase dependence on petroleum imports from foreign countries and also can increase air pollution from tailpipe emissions [1]. In addition, the price of vehicle fuels has been increasing at a significant rate in the U.S. and the environmental concerns from vehicle emissions (i.e. greenhouse gas emissions) have been increased in the past few years. Consequently, consumers have much more interest related to reducing fuel consumption of their vehicles and purchasing more fuel efficient and environmental friendly vehicles, for example, hybrid electric vehicles (HEVs). They are also interested in reducing fuel consumption in their own vehicles by fuel efficient driving techniques.

\subsection{Introduction of Eco-Driving Strategies}

In the last few years, vehicle drivers have been more interested in eco-driving due to increased petroleum based fuel price. In addition, the goal of many competitions and challenges held in North America and Europe is to achieve extremely low fuel consumption [2][3] by eco-driving strategies. There are many online sources $[4][5][6]$ which explain what eco-driving is and how 
to save more fuel on your own vehicle. Some common eco-driving rules on the real road are as follows;

- Anticipate traffic patterns

- Decelerate smoothly

- Minimize accessory loads such as air conditioning and heated seats

- Use kinetic energy (KE) for up and down hills

- Check tire pressure

- Close windows at high speed to reduce aero drag

Another possible strategy to reduce fuel consumption is to use the vehicle's fuel converter such as an engine to accelerate the vehicle to a certain high speed and then coast to a certain low speed with the engine off (or in neutral gear). This method will reduce fuel flow to zero (or idle fuel flow) during the coast phase. Also, the vehicle uses higher power engine load to accelerate to the upper vehicle speed in a limited time, thus increasing the engine brake thermal efficiency. This strategy is known as "pulse and glide" or "burn and coast" in some references [7][8].

\subsection{Research Objective}

The main objective of this study is to investigate and quantify vehicle inertia impact on fuel consumption for a conventional gasoline vehicle and an HEV using pulse and glide (PnG) driving strategy compared to steady speed driving case.

The specific objectives of this study are;

1. to estimate fuel consumption of two difference cases; the PnG and the steady speed cases at the same average speed for a conventional vehicle and an HEV

2. to quantify energy loss in a vehicle system (efficiency of energy storage in vehicle inertia) during acceleration (pulse) period

3. to identify effects of speed range, acceleration time and engine load on fuel consumption in the PnG driving strategy 
4. to identify interactions between PnG (vehicle inertia), HEV control strategies and battery energy storage

The following chapters will describe a literature review, preliminary study for the proposed driving strategy, simulation results and discuss the results from vehicle testing at the end of this dissertation. 


\section{Chapter 2 Literature Review}

\subsection{Overview of Pulse and Glide (PnG) Driving Strategy}

The basic idea of the PnG method is to put more kinetic energy (KE) into vehicle inertia (inertial energy) during acceleration phase and use that energy stored in vehicle inertia without additional energy input during coast phase. In acceleration of the PnG driving, the engine has to be operating in a higher load region and obviously consume more fuel than steady speed driving. However, the engine efficiency during acceleration becomes higher compared to the steady speed driving. Thus the overall principle of the PnG driving strategy is increasing engine load to accelerate the vehicle with higher engine efficiency in a relatively short period of time and using the stored KE during coast phase without further fuel consumption in the engine. The next two sections describe more details about the PnG driving strategy compared with steady speed driving.

\subsubsection{Description of PnG Driving Strategy}

The important point for the PnG driving strategy is that an engine must be off during coast phase. If an engine is on in coasting, the engine idles and produces minimum power to run accessories as shown in Figure 2.1-(a). It means that the engine still consumes fuel. In this case, it is very difficult to obtain the fuel consumption benefits from the PnG driving strategy. However, if engine is off in coasting, no more fuel is consumed until the vehicle slows down to a certain low speed as described in Figure 2.1-(b). This strategy is used in many vehicle competitions, for example, Society of Automotive Engineers (SAE) Supermileage [2]. Jawad, et al. estimated 
initial $(\sim 40 \mathrm{~km} / \mathrm{h})$ and final $(\sim 16 \mathrm{~km} / \mathrm{h})$ speeds during coasting, and 3.3 seconds of acceleration time for PnG driving strategy using relatively simple analysis based on the specifications of their small competition vehicle; $113 \mathrm{~kg}$ of vehicle mass [9]. In this analysis the engine operating time is for $5-10$ seconds which includes delay time of engine turning on manually. Note that the PnG cycle is very different from standard drive cycles where braking is much more common than coasting.

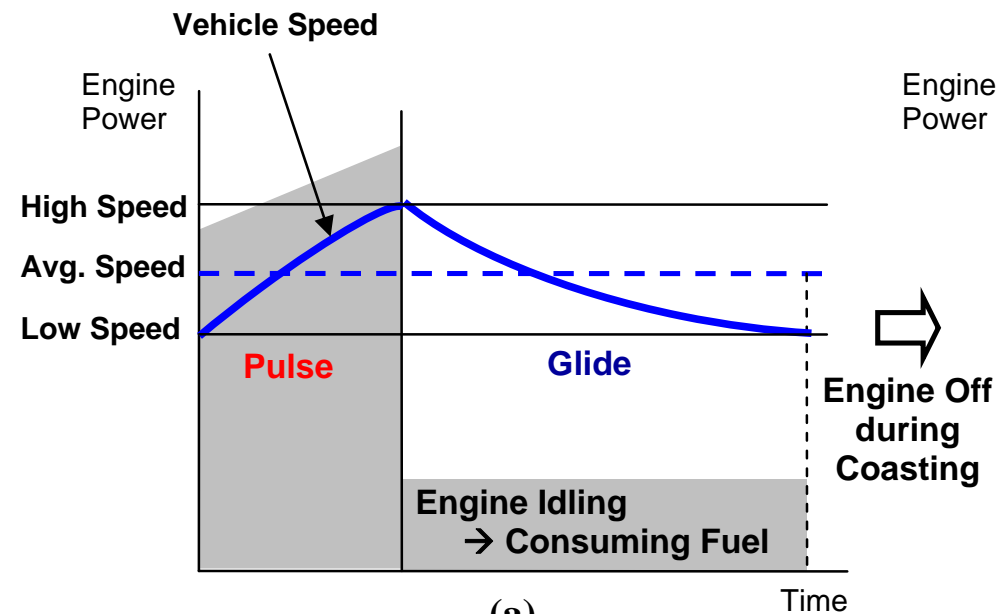

(a)

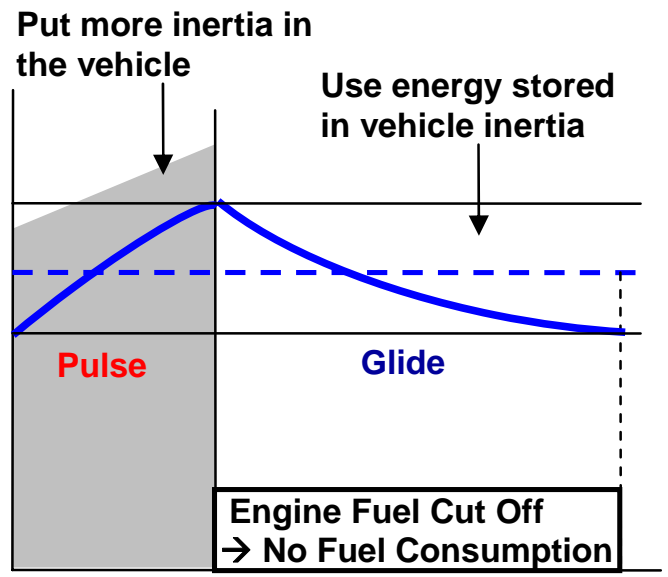

(b) Time

Figure 2.1 PnG with engine on (a) and engine off (b) during coasting

\subsubsection{Limitations of PnG Driving Strategy}

The PnG driving strategy can not be applied effectively to modern automatic transmission vehicles because of the torque converter lock up issue [10]. In general, an automatic transmission locks up the torque converter so the engine and the transmission are physically connected to each other to reduce power losses when the torque demand is low. However, the transmission unlocks the torque converter when the vehicle needs higher torque demand like higher acceleration. In this condition, power losses occur in the torque converter and it can possibly decreases the benefit of $\mathrm{PnG}$ driving strategy. In the preliminary testing for a conventional vehicle with a 5 speed automatic transmission, the results do not show fuel economy improvement by $\mathrm{PnG}$ driving compared to steady speed driving. However, conventional vehicles with a manual transmission do not have torque converter lock up so it is possible to apply the PnG driving strategy. 
Figure 2.2 shows how to operate a conventional vehicle with a manual transmission using the PnG strategy. In the beginning, the engine is on and the vehicle is in gear, so a driver should accelerate the vehicle to a certain high speed, turn off the engine to cut off fuel and disengage the gear for coasting. When the vehicle speed is close to a certain low speed, then the driver should turn on the engine again and put the vehicle in gear to accelerate the vehicle. In manual transmission vehicles, there are still a few limitations; turning engine on and off and gear shifting should be operated manually so it might cause a time delay. Also, lower or higher gear selection than a proper one along with the vehicle speed can cause engine jerk or engine stalling. Furthermore, there are known safety issues for manual transmission vehicles using the PnG driving strategy on the road; traffic consideration, unstable braking performance, and unstable power steering system if the engine is off.

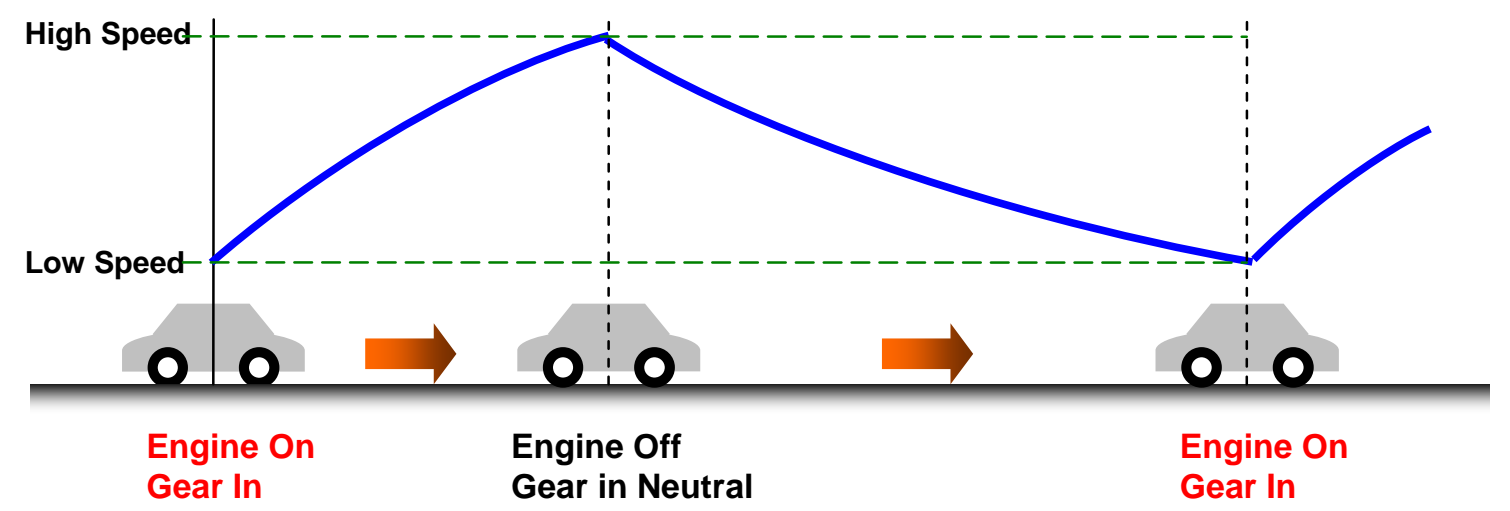

Figure 2.2 PnG driving strategy for conventional vehicles

\subsubsection{Kinetic Energy Use in Race Cars}

There are a few ways to recover $\mathrm{KE}$ of a vehicle and use it later. One example is a regenerative brake system commonly used in current HEVs. The other example could be the KE recovery systems (KERS) in race cars. A KERS has a flywheel to recover the KE as either electrical energy or mechanical energy during braking but it is of very limited use for racing cars because of its safety and instability. Recently, Formula One (F1) allowed use of a KERS with $60 \mathrm{~kW}$ maximum power and energy recovery per lap of $400 \mathrm{~kJ}$ in F1 racing cars for the 2009 season [11]. The KERS will be allowed for the 2010 season as well [12]. 
Cross simulated the KERS to optimize its performance for different racing tracks [13]. The results show that the lap time is improved between 0.27 and 0.46 seconds but he concludes that the benefit of the KERS is doubtful because the cars must carry the extra weight ( $24 \mathrm{~kg})$ of the flywheel. In addition, minor incidents (fire and electric shock) were reported recently due to the KERS.

\subsection{Impact Factors on Fuel Consumption of Vehicles}

There are many impact factors which affect fuel consumption of vehicles, for example, vehicle mass, aerodynamic drag, rolling resistance, driving patterns, fuel properties, road conditions, and so on. Some of them have very significant impact and others have minor impact. In this study, significant impact factors such as driving patterns (drive cycles and drivability) and vehicle mass are focused and studies related to those factors are reviewed.

\subsubsection{Drive Cycles and Drivability}

An interesting study was conducted by Sharer, et al. [14]. They scaled up and down the vehicle speed $(0.8,1$ and 1.2 times of the vehicle speed) in city and highway drive cycles and simulated a conventional vehicle (2004 Ford Focus) and an HEV (2004 Toyota Prius) to demonstrate the impact of drive cycle aggressiveness on fuel consumption. Scaling up the speed gives more aggressive acceleration and it directly increases inertial force which is mass times acceleration (see equation (3.7) in section 3.1). Therefore, the vehicle consumes more fuel than standard cases. The results show that the Prius is more sensitive in fuel economy than the Focus on city driving (UDDS: Urban Dynamometer Driving Schedule) when the vehicle speed is scaled up and down proportionally. It means that the increase of fuel consumption of the Prius is larger than that of the Focus as the drive cycle becomes more aggressive. Duoba, Lohse-Busch and Bohn completed another similar study for investigating the sensitivity of conventional and hybrid electric vehicles to driving patterns with less and more aggressive drive cycles prior to the previous study [15]. They also multiplied the vehicle speed of city and highway drive cycles by $0.8,1.0,1.2$ and 1.4 to make less and more aggressive driving conditions. They tested four 
different conventional vehicles and two different HEVs. Their results also show that the fuel economy of HEVs drops more than that of conventional vehicles, especially, on the city drive cycle (UDDS).

\subsubsection{Vehicle Mass and Inertia}

Research related to impact of vehicle mass or inertia on fuel consumption is not particularly for PnG but for other drive cycles. For example, An and Santini investigated mass impacts on fuel economy of conventional vehicles and HEVs for city and highway drive cycles [16]. They defined the mass-impact term which includes inertia to examine its effect on fuel consumption. According to the study, the mass-impact term on fuel consumption is very dominant for both drive cycles. Also, the study shows that the fuel economy change of HEVs due to vehicle mass is a little less sensitive than that of conventional vehicles. A similar study was completed by Reynolds and Kandlikar [17]. They compared HEVs to conventional vehicles to statistically determine how vehicle weight and system power can affect fuel consumption. In this study, it is found that the increase of fuel consumption due to increased vehicle weight in HEVs is lower than that in equivalent conventional vehicles, especially in city driving because HEVs can recover regenerative brake energy during frequent stop situations and save more fuel. However, the difference of fuel consumption penalty due to increased vehicle weight is relatively smaller in a highway driving condition because both vehicle types mostly use their internal combustion engines.

\subsection{HEV Control Strategy}

There are many studies for finding optimum HEV control strategies to minimize energy consumption among engine, motor and battery for HEVs including the vehicle used in this study, 2004 Toyota Prius. HEV control strategies are very broad, hence the literature of few selected areas are reviewed and summarized in this section. 


\subsubsection{System Control of an HEV}

Four power-split HEVs were tested on a chassis dynamometer for UDDS and US06 (more aggressive driving pattern) cycles to analyze their control strategies by researchers at Argonne National Laboratory (ANL) [18]. The tested vehicles include a 2004 Toyota Prius. The results describe that the 2004 Toyota Prius has a well sized engine for UDDS driving and the engine operation is distinctively different from other HEVs to charge the battery at low engine-power conditions. In other words, the engine in a 2004 Toyota Prius does engine load leveling in high efficiency region with the battery instead of operating in low efficiency region.

Sezer, et al. introduced a control strategy, "maximizing overall efficiency strategy (MOES)," for an HEV [19]. As illustrated in Figure 2.3, the consumed fuel power represents input and the power transferred to road represents output. The MOES searches for an optimum point to maximize the overall system efficiency of the vehicle, $\eta_{S Y S}=\frac{P_{\text {road }}}{P_{\text {fuel }}}$, with a given driving condition. In the vehicle system, they considered component efficiencies and separated MOES to two modes; charge and discharge conditions. The developed MOES was applied to an HEV in the ECE 15 cycle (a European urban drive cycle) to achieve approximately $16 \%$ of reduction of fuel consumption.

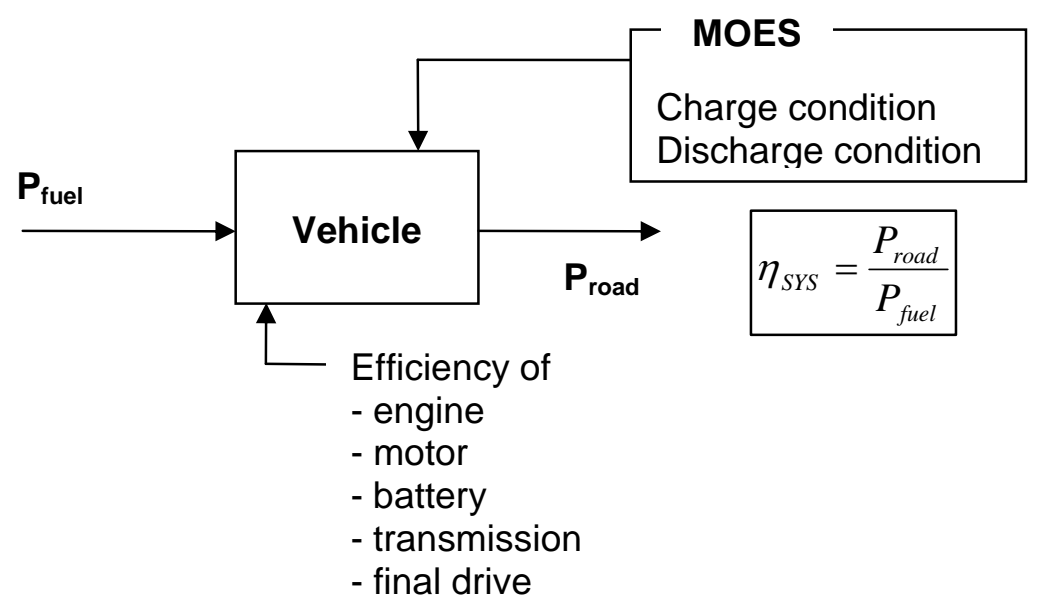

Figure 2.3 Basic concept of MOES as a control strategy for an HEV 
Liu and Peng developed an algorithm, equivalent consumption minimization strategy (ECMS) to improve fuel economy of a power-split HEV which is the same vehicle configuration as the Toyota Hybrid System (THS) [20]. The ECMS is based on the concept of instantaneous equivalent fuel consumption which is related to the SOC of the battery. Hence, they defined the SOC weighting factor and adopted a dynamic programming technique for improving the ECMS algorithm. The basic idea of this approach is to find the optimum point to minimize fuel consumption by controlling available power between engine and battery along with SOC.

In other papers, the instantaneous system efficiency based on the charging and discharging states of the battery was defined and employed to reduce overall fuel consumption of a series-parallel HEV [21]. The load leveling map at the ring gear (output shaft) and the e-CVT (electronic continuous variable transmission) control map are also made to improve HEV control strategy.

\subsubsection{Control Strategy Based on Power Loss Calculation}

Boyd and Nelson used a little different approach which is power loss calculation method to find an operational control strategy for HEVs [22][23]. They calculated individual powertrain component losses for engine, transmission, final drive, motors and battery, and compared them with possible operational cases. A similar method was used by Johnson for calculating losses in different hybrid operational modes in a plug-in hybrid electric vehicle (PHEV) [24]. Baglione, et. al. developed a tool to analyze energy losses in vehicle systems so they can investigate vehicle system energy requirements for various drive cycles [25]. In this study, accessory loads are considered by a more accurate approach in their tool as well.

\subsubsection{Control Strategy Using Driving Pattern Recognition}

The other interesting approach to control of a HEV system is the driving pattern recognition (DPR) method proposed in few references [26][27]. In the two studies, six representative driving patterns were selected; three urban, one expressway and two suburban driving patterns. Those driving patterns were analyzed in terms of 24 parameters such as vehicle speed, acceleration, grade, time and so on. Once the current driving pattern is analyzed by either neural network [26] or the dynamic programming for their DPR method [27] and recognized as one of six driving 
patterns, the vehicle switches the control strategy to an optimal one to reduce fuel consumption. It is briefly illustrated in Figure 2.4.

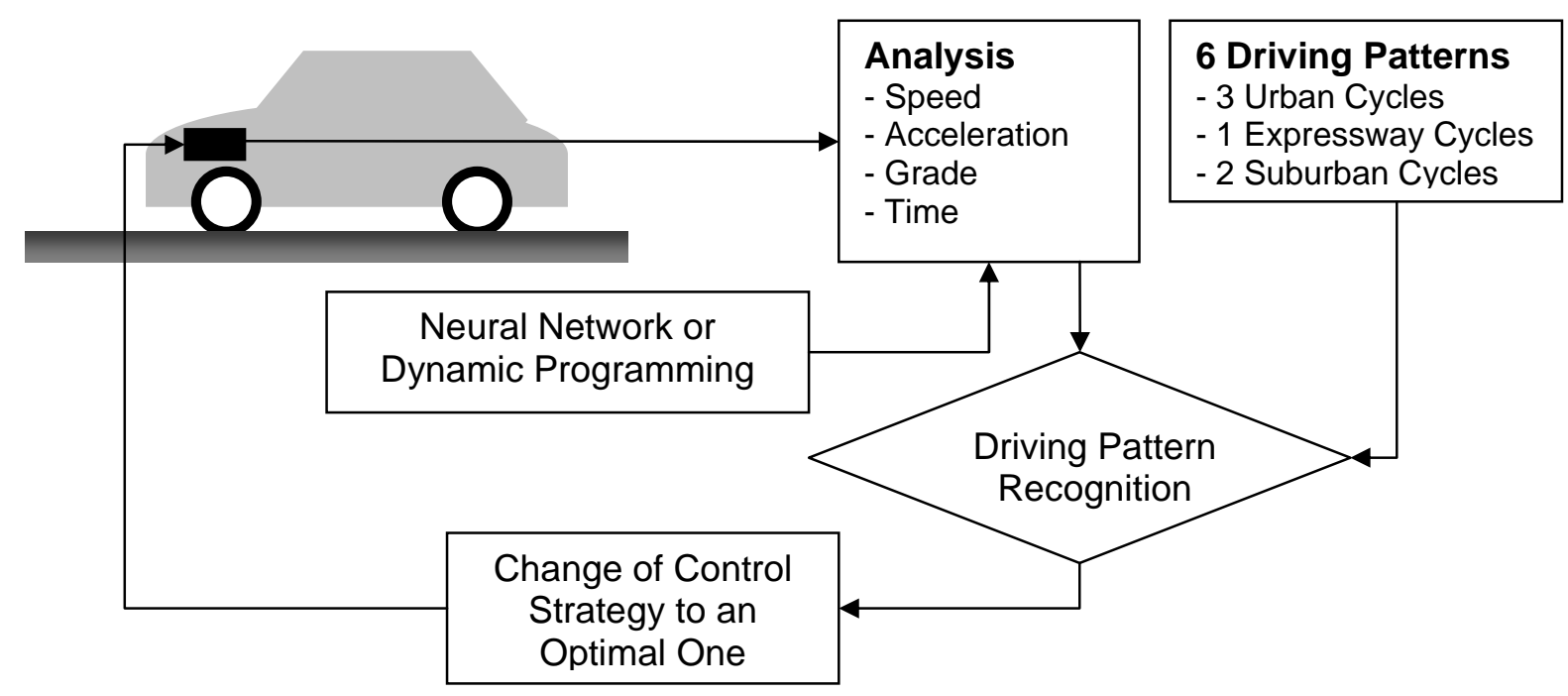

Figure 2.4 Schematic of a HEV control strategy using driving pattern recognition (DPR) method

\subsubsection{Control of Engine Start / Stop}

One of key technologies used to improve fuel economy of HEVs is the engine start / stop system. This engine control strategy has been used in few HEVs currently available on the market [28]. The main purpose of controlling the engine start / stop is to cut off unnecessary fueling and save fuel when the vehicle is stopped or in idling. The benefit of the system is most significant for a vehicle which has frequent stops, for example, urban buses. Bass and Alfermann investigated the influence of idling in a few drive cycles on fuel economy of urban hybrid electric buses and tested them on a dynamometer [29]. It is clearly found that the benefit of fuel economy (percent of fuel economy improvement) by the engine start / stop system is increased as the stopping frequency is increased. A similar engine start / stop system was integrated in a mild HEV and tested [30]. Bishop, et al. integrated it with a starter / alternator in the vehicle and tested it on a chassis dynamometer for city and highway drive cycles. The results indicate $5.3 \%$ and $4 \%$ of fuel economy improvement for the city and highway drive cycles respectively. 
The experiment of idling stop was even conducted on a conventional gasoline vehicle for studying its effect on fuel consumption by Matsuura, Korematsu and Tanaka [31]. They manually stopped the engine when the vehicle was stopped and restarted it when the vehicle was launched. Their results show that the fuel consumption by idling stop is reduced by $4-8.7 \%$ depending on frequency and time of idling stop. As a matter of fact, most states in the U.S. have regulations to reduce unnecessary engine idling of vehicles due to environmental concerns [32].

As summarized in this section, most studies about HEV control strategy used standard drive cycles which have braking event. However, the PnG driving strategy does not include braking part so one can closely investigate the interaction between the engine and the battery energy storage, for example, engine load leveling with the battery in an HEV.

\subsection{Planetary Gear Set in HEV}

A general planetary gear set consists of a ring gear, a carrier, a sun gear and pinions gears. This gear set has been used in some of HEVs, especially Toyota Prius [33][34]. The 2004 Toyota Prius uses a planetary gear set as a transmission which is referred as a power split device (PSD) or called an e-CVT (electric continuously variable transmission). The PSD used in 2004 Toyota Prius is schematically demonstrated with an engine and motor/generators and the driveline in Figure 2.5. The torque and power of the engine and the two motor/generators is combined and split by the PSD. The carrier is connected to the engine so it transmits the engine torque/power to the planetary gear set. The sun gear is connected to the MG1 (mostly a generator) and it generally controls the speed of other components in the planetary gear set. The ring gear is connected to the MG2 (mostly a traction motor) and the torque/power of the engine and the MG2 is combined here to be transmitted to driveline. Also the speed of the ring gear is directly related to the vehicle speed with a certain speed ratio (reduction gear and final drive). More details about speed, torque and power of a planetary gear set are summarized in Appendix A.

Miesel did an analytical study for the single-mode hybrid electric powertrain of 2004 Toyota Prius [35] and the two-mode hybrid electric powertrain [36]. He derived equations for speed, power and torque of the planetary gear set and introduced a dimensionless parameter, called a 
separation factor, which plays a key role in the PSD of the vehicle. However, the efficiency of the planetary gear set was not investigated. Cho, Ahn and Lee also derived equations of the PSD analytically and defined a key parameter, a speed ratio, which is similar to the separation factor [37]. In their paper, they carried out the transmission efficiency of the planetary gear in an HEV powertrain. In addition, they used their efficiency model of the powertrain to obtain the system optimal point with respect to fuel consumption including engine operation [38].

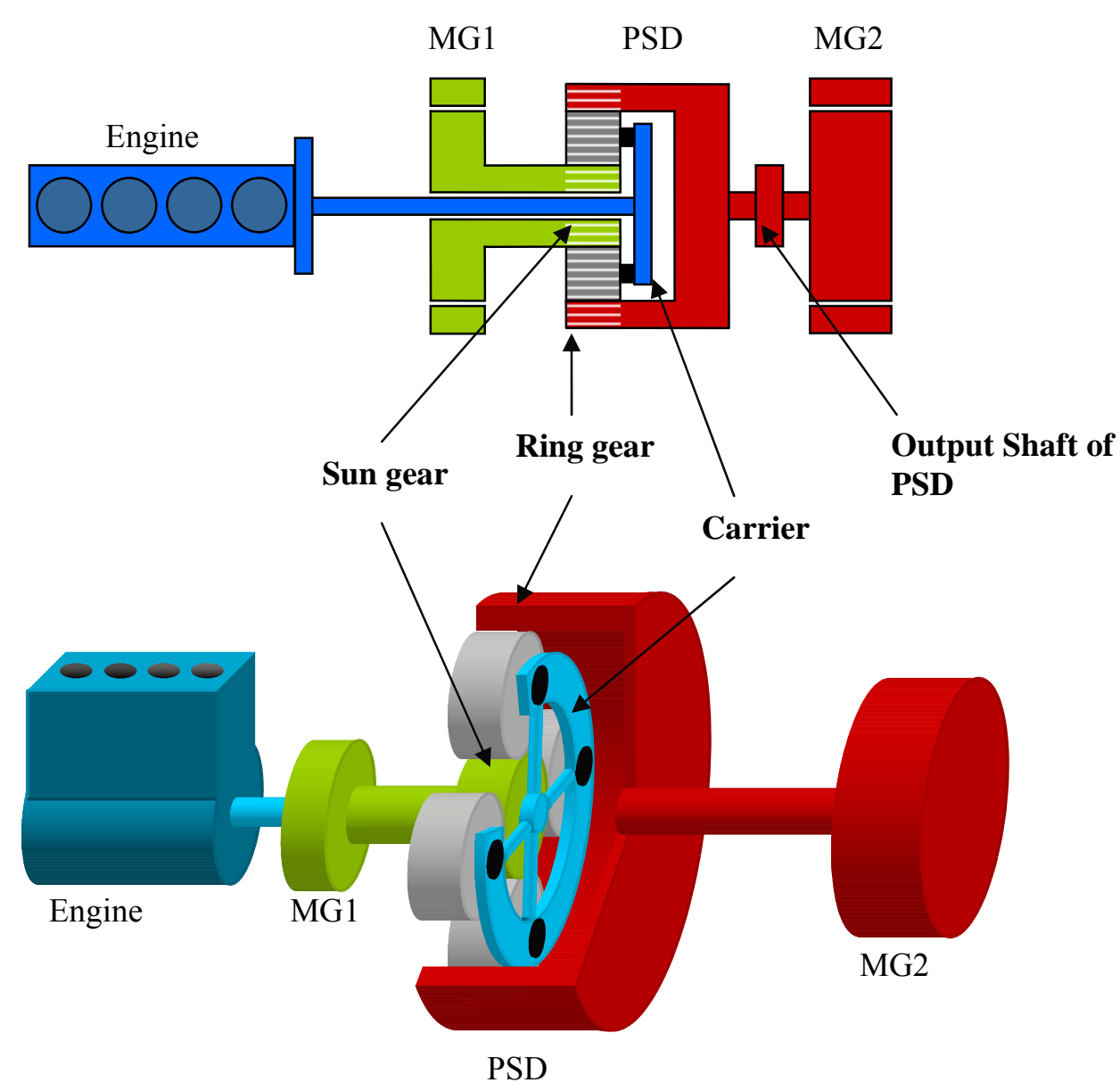

Figure 2.5 Driveline with the power split device (PSD) in 2004 Toyota Prius

Note that the PSD used in 2004 Toyota Prius has speed limits for each gear by its own control strategy ; MG1: $\pm 10,000 \mathrm{rpm}, \mathrm{MG} 2: 0 \sim 6,500 \mathrm{rpm}$, and engine: $0 \sim 5,000 \mathrm{rpm}$ respectively [33]. The speed of one gear is constrained by equation (A.1) in Appendix A if the speeds of other two gears are specified. 


\subsection{Concerns for Testing Hybrid Electric Vehicles (HEVs)}

In general, HEVs charge the battery by engine or regenerative brake system and discharge the battery whenever they need additional power to propel. In this process, the SOC can vary and it affects fuel consumption. For example, if a final SOC is larger than an initial SOC for a given drive cycle, it means that the vehicle probably consumed more fuel than for a charge balanced condition. Therefore, correction of SOC after a simulation or test of HEVs has been an issue to obtain accurate fuel consumption. The SOC correction method was developed by Duoba and Larsen [39] for an advanced vehicle competition, the 1995 HEV challenge, over a decade ago. They plotted the data as fuel used vs. normalized charge to obtain SOC corrected fuel consumption as schematically illustrated in Figure 2.6-(a). In addition, the SOC correction is documented in the Society of Automotive Engineers (SAE) as a recommend procedure for testing HEVs [40]. According to the document, the change of initial and final SOC over a drive cycle should be within $\pm 1 \%$.

A similar approach was used in the study for hybrid electric and conventional transit buses [41]. The fuel economy from vehicle testing is plotted over the change in battery SOC to get accurate results. Also, Lohse-Busch, et al. plotted the fuel consumption (L/100 km) data over the energy consumption (Wh/mile) in their vehicle testing for a modular automotive hybrid test bed [42] as schematically described in Figure 2.6-(b).

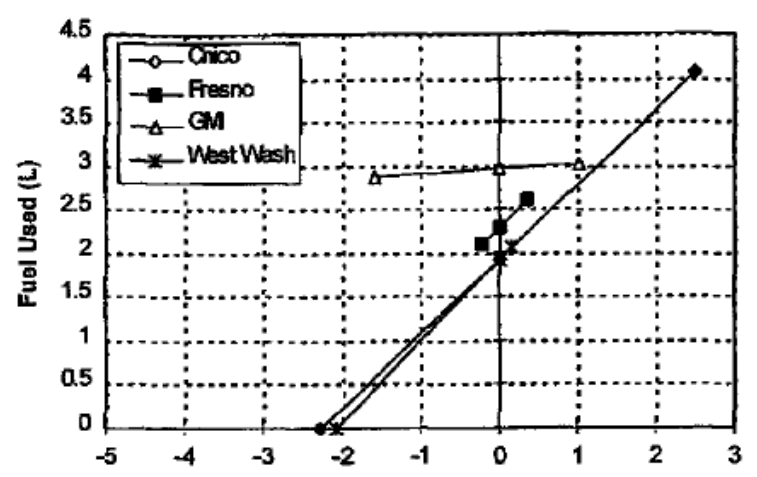

(a) SOC corrections for Saturn and Neon class HEVs [39]

(Reprinted with permission from SAE paper number 960740 (c) 1996 SAE International)

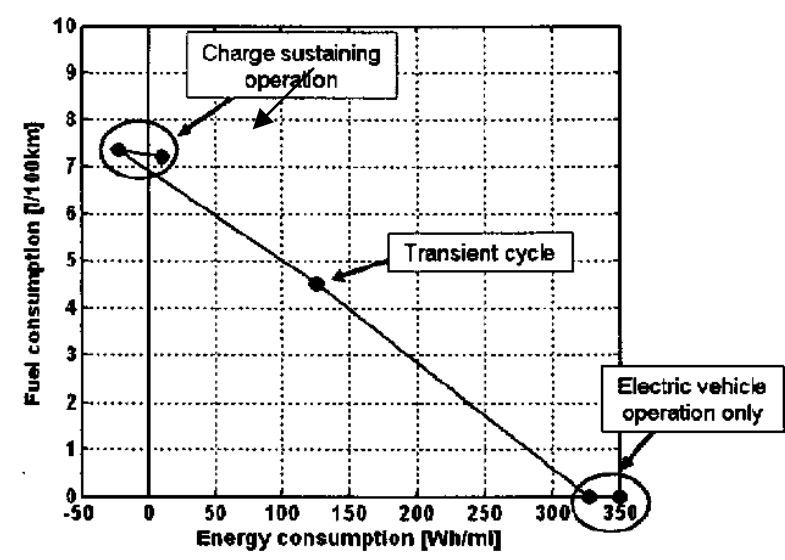

(b) Energy and fuel consumption graph for the engine optimum plug-in hybrid test [42] (Reprinted with permission from SAE paper number 2009-01-1315 (c) 2009 SAE International)

Figure 2.6 Examples of SOC correction method 
As described in this section, the fuel consumption of HEVs should be carefully calculated with SOC correction. Otherwise, the results can vary for the same vehicle in the same drive cycle.

\subsection{Research Contribution}

In previous sections of Chapter 2, many references are reviewed and summarized. There are a few articles about reducing fuel consumption using the PnG driving strategy, but deep analysis for the PnG driving strategy had been barely done until now. Most of those articles are documented by vehicle owners with their own real life experiments. Therefore, this study will primarily focus on investigating and quantifying the impact of kinetic energy stored in vehicle inertia on fuel consumption of conventional vehicles and HEVs using the PnG driving strategy compared to a steady speed case. Furthermore, this study will identify interactions of storing energy between vehicle inertia and battery by HEV control strategies through the PnG drive cycles and document which is the more efficient way to store energy and use it later. This approach is unique because it uses a particular drive cycle, the $\mathrm{PnG}$, which is very different from standard drive cycles where braking is much more common than coasting. Most of the research for improving HEV control strategies is conducted with standard drive cycles so they cannot show the impact of KE on fuel consumption during coasting.

The results from this study can also possibly contribute to improved HEV control strategies for better fuel economy when an HEV is driven by an adaptive cruise control system with a global positioning system (GPS) or the vehicle is running on repeated up and down hills. 


\section{Chapter 3 Simple Vehicle Modeling and Parametric Study for a Conventional Vehicle}

In the beginning of this study, a simple spread sheet model is developed to simulate a conventional vehicle with a five speed manual transmission for both PnG and steady speed cases. A few assumptions are made for this model to clearly understand how PnG driving strategy works and what kind of parameters can possibly affect fuel consumption of a conventional vehicle compared to steady speed driving. In this chapter, the equations and the assumptions used in the simple vehicle model are described in detail. In addition, the efficiency of KE storage is defined to find how efficient it is to store $\mathrm{KE}$ and use it later by $\mathrm{PnG}$ driving strategy. Finally, the results of a parametric study for a conventional vehicle are fully discussed in later sections of this chapter.

\subsection{Tractive Force at Wheels}

The fundamental knowledge about vehicle performance analysis is well described in many text books and some references [43][44][45][46]. In this section, forces acting on a vehicle in the direction of acceleration are defined and each term is explained briefly. 


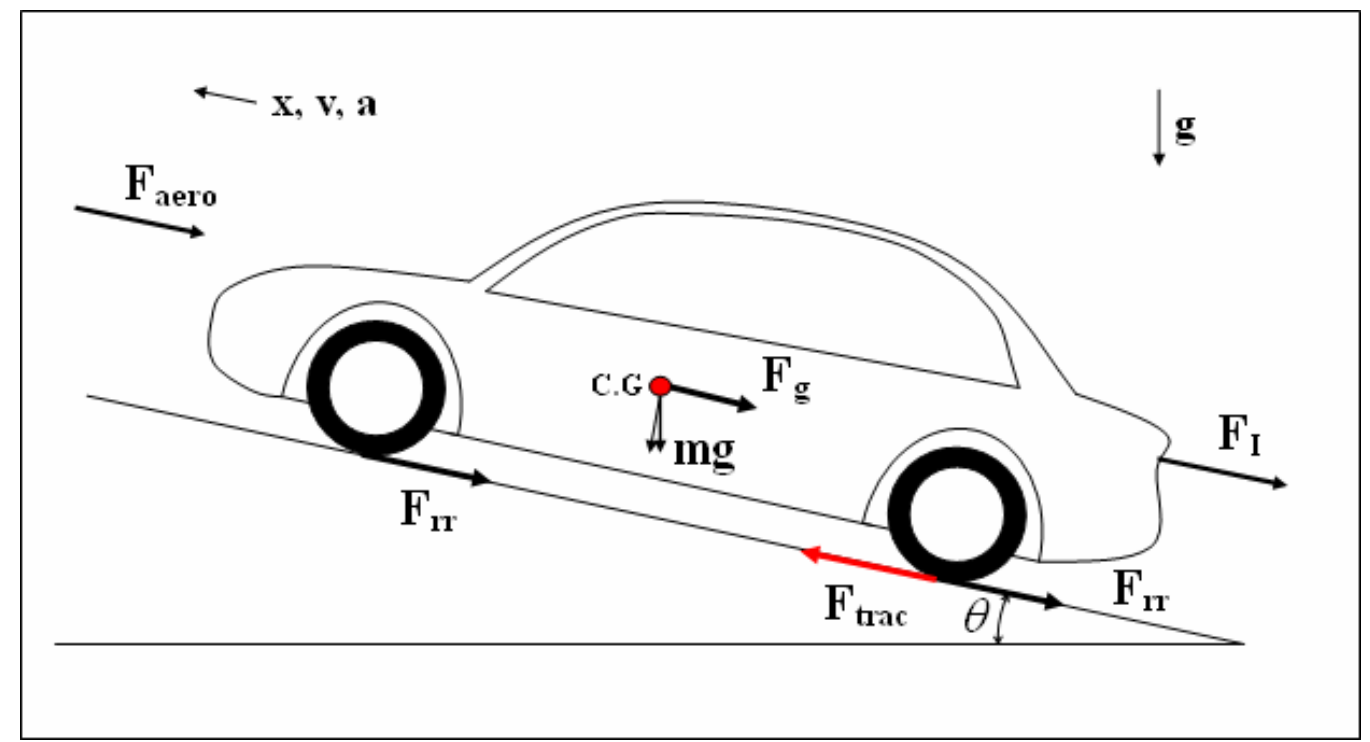

Figure 3.1 Diagram of forces acting on a vehicle

Equation (3.1) represents the aerodynamic drag force which is proportional to the air drag coefficient, the frontal area of the vehicle and the square of the vehicle speed as well. A larger frontal area and higher vehicle speed increase the aerodynamic drag resistance.

$$
F_{\text {aero }}=\frac{1}{2} \rho C_{D} A V^{2}
$$

Where: $F_{\text {aero }}$ : Aerodynamic drag force $(\mathrm{N})$

$\rho$ : Density of air $\left(\sim 1.2 \mathrm{~kg} / \mathrm{m}^{3}\right)$

$C_{D}$ : Aerodynamic drag coefficient

$A_{f}$ : Frontal area of a vehicle $\left(\mathrm{m}^{2}\right)$

$V:$ Vehicle speed $(\mathrm{m} / \mathrm{s})$

The rolling resistance can be simply expressed as equation (3.2).

$$
F_{r r}=C_{r r} m g \cos \theta
$$

Where: $F_{r r}$ : Rolling resistance $(\mathrm{N})$

$C_{r r}$ : Rolling resistance coefficient

$m$ : Total mass of test vehicle $(\mathrm{kg})$

$g:$ Acceleration of gravity $\left(\mathrm{m} / \mathrm{s}^{2}\right)$

$\theta:$ Angle of the road from horizontal (rad)

Equation (3.3) is the grade force due to the angle of the road. 


$$
F_{\text {grade }}=m g \sin \theta
$$

Where: $F_{\text {grade }}:$ Grade force due to the angle of the road $(\mathrm{N})$

If the vehicle is in a steady speed condition (no acceleration), the forces acting on the vehicle are simply the sum of equations (3.1), (3.2) and (3.3). If there is no grade on the road, the road load can be expressed as shown in equation (3.4)

$$
F_{\text {Road Load }}=F_{\text {aero }}+F_{r r}
$$

If road load coefficients of a vehicle are known, equation (3.4) for road load above can be also expressed as shown below. The road load coefficients in equation (3.5) can be obtained by curve fitting of road load forces from a coast down (no propelling or braking forces) testing on a flat road. Each term in equation (3.5) cannot be directly equated to the terms in equation (3.4) because equation (3.5) is derived from curve fitting of coast down data. However, the $C_{D} A_{f}$ and $C_{r r}$ terms in equation (3.1) corresponds to the coefficients $C$ and $A$ in equation (3.5) respectively. Note that the coefficient B cannot be directly related to the terms in equation (3.4) because there is no term in that equation which is proportional to vehicle speed.

$$
F_{\text {Road Load }}=A+B V+C V^{2}
$$

Where: $A$ : Road load coefficient $(\mathrm{N})$

$B$ : Road load coefficient $(\mathrm{N} /(\mathrm{m} / \mathrm{s}))$

$C:$ Road load coefficient $\left(\mathrm{N} /(\mathrm{m} / \mathrm{s})^{2}\right)$

For PnG cases, it is necessary to separate one cycle into two parts; acceleration and coast phases because the tractive forces at wheels for each part are different. The next sections briefly explain about separated PnG and steady speed cases.

\subsubsection{Acceleration (Pulse) Phase}

In general, the equation of motion of a vehicle with acceleration along the $\mathrm{x}$-axis (longitudinal direction) is given by 


$$
\begin{aligned}
& m_{I} a=F_{\text {trac, wheel }}-F_{\text {Road Load }} \\
& \Rightarrow m_{I} a=F_{\text {trac, wheel }}-F_{\text {aero }}-F_{r r}-F_{\text {grade }}
\end{aligned}
$$

Where: $F_{\text {trac,wheel }}$ : Tractive force at driven wheels $(\mathrm{N})$

$m_{I}$ : Effective mass of a vehicle $(\sim 1.03 \times \boldsymbol{m})(\mathrm{kg})$

$a:$ Longitudinal acceleration $\left(\mathrm{m} / \mathrm{s}^{2}\right)$

The left term in equation (3.6) represents inertial force due to acceleration as shown in equation (3.7)

$$
F_{I}=m_{I} a
$$

Where: $F_{I}:$ Inertial force due to acceleration $(\mathrm{N})$

In other words, equation (3.6) could be rearranged in terms of the tractive force which can propel the vehicle. Also if it is assumed that a vehicle is on a level road, equation (3.6) can be simplified as shown below due to zero grade force.

$$
F_{\text {trac, wheel }}=F_{\text {aero }}+F_{r r}+m_{I} a
$$

The required tractive power at driven wheels can be simply calculated by multiplying vehicle speed to equation (3.8).

$$
\begin{aligned}
P_{\text {trac,wheel }} & =F_{\text {trac,wheel }} V \\
& =\left(F_{\text {aero }}+F_{r r}+m_{I} a\right) V
\end{aligned}
$$

Where: $P_{\text {trac, wheel }}$ : Tractive power at driven wheels (W)

\subsubsection{Coast (Glide) Phase}

In coast phase, the engine power is disconnected from transmission, thus there is no tractive force at wheels, $F_{\text {trac,wheel }}=0$ and equation (3.8) becomes

$$
\begin{aligned}
& 0=F_{\text {aero }}+F_{r r}+F_{I} \\
& \Rightarrow F_{I}=-\left(F_{\text {aero }}+F_{r r}\right)
\end{aligned}
$$


Equation (3.10) represents the inertial force during deceleration as vehicle $\mathrm{KE}$ is discharged to overcome the road load during coasting.

\subsubsection{Steady Speed}

If the vehicle is at a steady speed, there is no inertial force due to acceleration, so equation (3.9) becomes

$$
P_{\text {trac, wheel }}=\left(F_{\text {aero }}+F_{r r}\right) V
$$

\subsection{Simple Vehicle Modeling for a Conventional Vehicle}

A simple backward tracking model which can generate fuel economy of a conventional vehicle is made to determine speed ranges of $\mathrm{PnG}$ drive cycles using equations described in previous section and some references [43][46][47][48]. Figure 3.2 illustrates a schematic of the simple vehicle model for a conventional vehicle. In Figure 3.2, the model mainly consists of two parts; the vehicle model and the wheel model. First, the vehicle model calculates engine fuel consumption with engine speed, engine torque and power, and engine overall efficiency. It determines the required tractive power through the driveline for the wheel model as well. Secondly, the wheel model calculates the acceleration and updates the vehicle speed. In addition, the wheel model can be independently used to generate a speed profile of a PnG case without calculating fuel consumption.

In this simple vehicle model, constant values of engine torque are used for each case to represent a constant driver's demand conveniently and to simplify the model so it can make effects of other parameters straightforward. From the given constant engine torque and initial vehicle speed, the model selects a proper gear and calculates required values to update vehicle speed. After one case is run, the model compares the calculated acceleration time to a given acceleration time. If they are not within a small error $(\sim 0.05 \mathrm{mph})$, the given constant engine torque should be changed and the model runs again until the vehicle can achieve the given acceleration time. More details are described with equations in the following sections. 


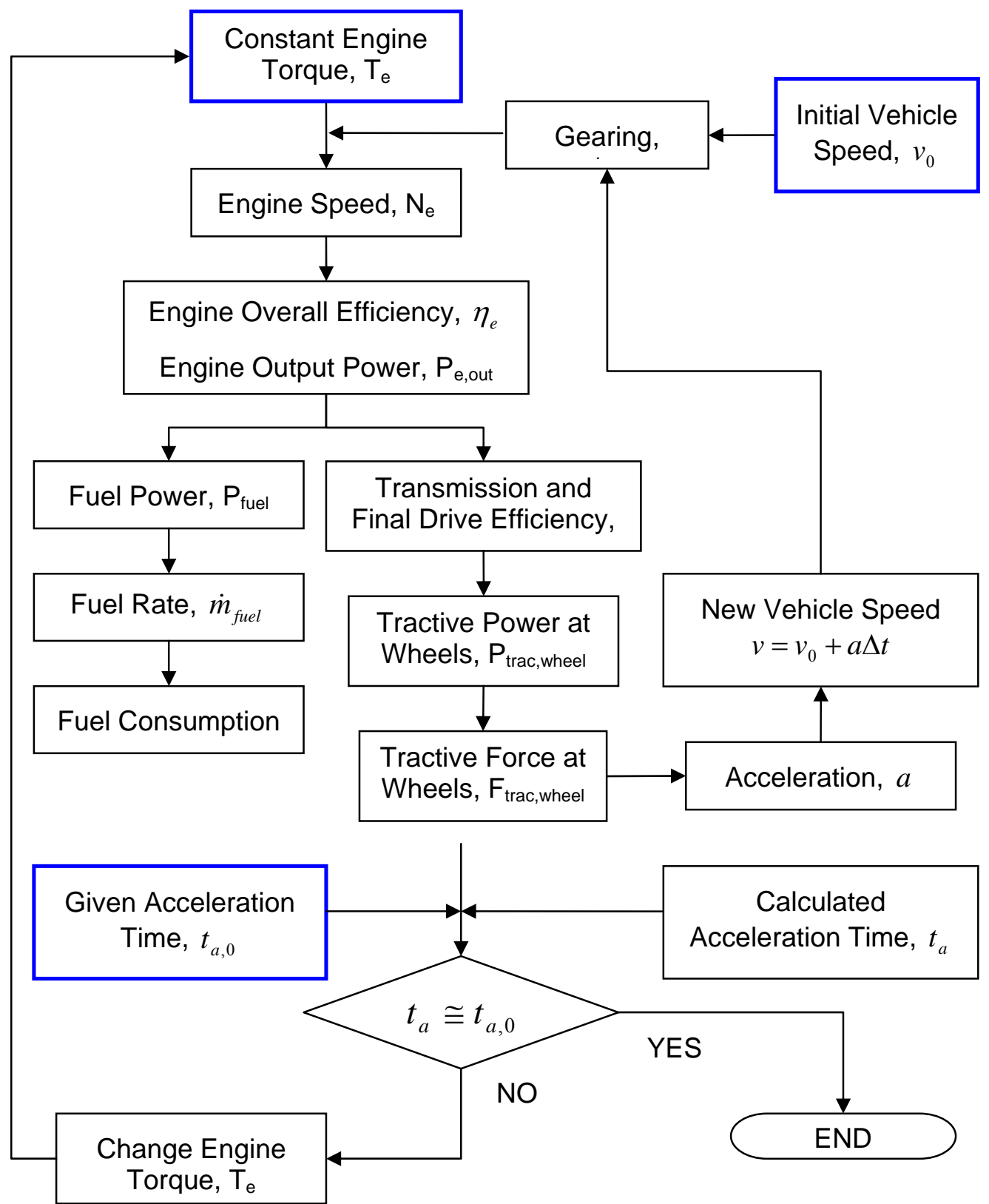

Figure 3.2 Schematic of the simple vehicle modeling for a conventional vehicle 


\subsubsection{Acceleration (Pulse) Phase}

Let's assume that the engine produces constant torque, $T_{e, \text { out }}$, in acceleration. Engine output power, $P_{e, \text { out }}$, is simply defined with the given $T_{e, \text { out }}$ below.

$$
P_{e, \text { out }}=2 \pi \mathrm{N}_{e} T_{e, \text { out }}
$$

Where: $P_{e, \text { out }}$ : Engine output power (W)

$T_{e, \text { out }}:$ Engine output torque $(\mathrm{Nm})$

$N_{e}$ : Engine speed (rev/s)

The gear should be properly selected based on vehicle speed and limit of engine torque and power, otherwise either the vehicle cannot follow the speed or the engine is operating at higher speed than required which can result in higher fuel consumption. Once the proper gear is selected, the engine speed in equation (3.12) can be determined by a given vehicle speed, $V$.

$$
N_{e}=\left(\frac{N}{V}\right)_{\text {top }}\left(\frac{g}{g_{\text {top }}}\right) V_{m p h}
$$

Where: $\left(\frac{N}{V}\right)_{\text {top }}$ : Engine speed (rpm) to vehicle speed (mph) ratio in top gear (rpm/mph)

$$
\begin{aligned}
& \left(\frac{g}{g_{\text {top }}}\right): \text { Normalized gear ratios at each gear by top gear ratio } \\
& V_{m p h}: \text { Vehicle speed }(\mathrm{mph})
\end{aligned}
$$

The general value of a small size car for $\left(\frac{N}{V}\right)_{\text {top }}$ is 35.6 [47]. Equation (3.14) below shows tractive power at wheels which is transmitted from the engine through the transmission and final drive in acceleration.

$$
P_{\text {trac, wheel }}=\eta_{t f} P_{e, \text { out }}=\left(F_{\text {aero }}+F_{r r}+m_{I} a\right) V
$$

Where: $\eta_{t f}$ : Combined efficiency of transmission and final drive 
The value of $P_{\text {trac,wheel }}$ should be the same as the value that calculated from equation (3.8) for a given vehicle speed. The power loss in driveline can be expressed by using equations (3.8) and (3.14).

$$
\begin{aligned}
P_{t f, \text { loss }} & =\left(1-\eta_{t f}\right) P_{e, \text { out }}=P_{e, \text { out }}-P_{\text {trac }, \text { wheel }} \\
& =P_{e, \text { out }}-\left(F_{\text {aero }}+F_{r r}+F_{I}\right) V
\end{aligned}
$$

Where: $P_{t f, \text { loss }}:$ Power loss in driveline (W)

In order to find the fuel rate of the vehicle, engine overall efficiency and power of fuel should be obtained. The engine overall efficiency can be obtained from equation (3.16) which is simplified from the study done by Nam and Sorab [49].

$$
\eta_{e}=\eta_{\text {th }} \frac{1}{1+\left(\frac{f m e p_{0} V_{d}}{4 \pi T_{e, o u t}}\right)}
$$

Where: $\eta_{e}$ : Engine overall efficiency (\%)

$\eta_{t h}:$ Indicated thermal efficiency of engine ( $40 \%$ for gasoline engine) fmep $_{0}$ : Friction mean effective pressure at idling $(\mathrm{kPa})$

$V_{d}$ : Engine displacement (L)

The engine overall efficiency equation above does not include any friction term related to engine speed but constant friction mean effective pressure (fmep) at idling so engine efficiency remains constant at a given engine torque. This simple approach is useful for normal engine operating conditions because most of gasoline engines do not have significant change of efficiency by fmep at a given engine torque in the operating region used in this study [49].

Note that the simplified engine efficiency model here is not valid in high engine speed range because friction loss does not increase as engine speed is increased. Also, the model does not take account enrichment so it is not valid around maximum torque region. However, this simplified engine efficiency model is acceptable because the engine is operating in neither high speed range nor maximum engine torque region for both PnG and steady speed cases.

Next, the power of fuel into engine can be calculated with engine overall efficiency obtained from equation (3.16). 


$$
P_{\text {fuel }}=\frac{1}{\eta_{e}} P_{e, \text { out }}
$$

Where: $P_{\text {fuel }}$ : Power of fuel into engine $(\mathrm{W})$

The fuel rate can be calculated by using a lower heating value (LHV) of fuel and the power of fuel from equation (3.17).

$$
\begin{aligned}
\dot{m}_{\text {fuel }} & =\frac{P_{\text {fuel }}}{L H V} \\
& =\frac{P_{e, \text { out }}}{\eta_{e} L H V}=\frac{P_{\text {trac, wheel }}}{\eta_{e} \eta_{t f} L H V}=\frac{1}{\eta_{e} \eta_{t f} L H V}\left(F_{\text {aero }}+F_{r r}+F_{I}\right) V
\end{aligned}
$$

Where: $\dot{m}_{\text {fuel }}:$ Fuel rate into engine $(\mathrm{g} / \mathrm{s})$

$L H V$ : Lower heating value of fuel $(\mathrm{kJ} / \mathrm{g})$, (gasoline $\sim 42.8 \mathrm{~kJ} / \mathrm{g}$ )

For the power loss in engine due to heat and friction, equation (3.19) is used.

$$
\begin{aligned}
P_{e, \text { loss }} & =\left(1-\eta_{e}\right) P_{\text {fuel }}=P_{\text {fuel }}-P_{e, \text { out }} \\
& =P_{\text {fuel }}-\frac{1}{\eta_{t f}}\left(F_{\text {aero }}+F_{r r}+F_{i}\right) V
\end{aligned}
$$

Where: $P_{e, \text { loss }}$ : Power loss in engine (W)

In order to generate a vehicle speed profile of PnG cases, acceleration of the vehicle should be calculated. From equations (3.9) and (3.14), acceleration (>0) is derived as shown below.

$$
a=\frac{1}{m_{i}}\left(\frac{\eta_{\text {tf }} P_{e, \text { out }}}{V}-F_{\text {aero }}-F_{r r}\right)
$$

The vehicle speed can be simply obtained by using a given vehicle speed and acceleration from equation (3.20). Note that the aerodynamic drag force varies with vehicle speed, so that the acceleration and vehicle speed need to be updated at discrete time intervals.

$$
V=V_{0}+a \Delta t
$$

Where: $V_{0}:$ Initial vehicle speed $(\mathrm{m} / \mathrm{s})$

$\Delta t:$ Time difference $(\mathrm{sec})$ 


\subsubsection{Coast (Glide) Phase}

When the vehicle has reached a maximum speed limit of a PnG case, the engine power should be disconnected from the transmission for true coasting with fuel cut off. Therefore the tractive force at wheels becomes zero; $F_{\text {trac,wheel }}=0$ (neglecting any wheel bearing, axle, and final drive drag). From equation (3.8), acceleration $(<0$, deceleration) can be obtained as shown below.

$$
\begin{gathered}
0=F_{\text {aero }}+F_{r r}+F_{i} \Rightarrow F_{i}=-\left(F_{\text {aero }}+F_{r r}\right) \Rightarrow m_{i} a=-\left(F_{\text {aero }}+F_{r r}\right) \\
a=-\frac{1}{m_{i}}\left(F_{\text {aero }}+F_{r r}\right)
\end{gathered}
$$

Again, the vehicle speed can be calculated from equation (3.21) with acceleration obtained from equation (3.22). Note that the engine is off, the gear is disengaged and the vehicle slows down with its stored KE, so it is not necessary to estimate power and losses in the engine for a conventional vehicle; $P_{e, \text { out }}=P_{\text {fuel }}=P_{e, \text { loss }}=P_{\text {tf loss }}=0$.

\subsubsection{Steady Speed}

In PnG driving cases described previously, there is positive acceleration in the pulse phase and negative acceleration in the glide phase. In contrast to that, steady speed cases have zero acceleration, so the vehicle inertial force becomes zero, $F_{I}=0$. For this steady speed condition, it is simpler than PnG to derive the equations as explained below.

The engine output power and engine speed of a steady speed case can be easily calculated by using equations (3.12) and (3.13) respectively with a constant engine torque for a given vehicle speed equal to the average vehicle speed from a PnG case. The tractive power at wheels in steady speed with respect to engine output power is shown in equation (3.23). However, the value of $P_{\text {trac,wheel }}$ should be the same as the value from equation (3.14) rather than (3.8) due to zero inertial force for this case.

$$
P_{\text {trac,wheel }}=\eta_{t f} P_{e, \text { out }}=\left(F_{\text {aero }}+F_{r r}\right) V
$$


Again, the power loss in driveline is slightly different from equation (3.15) and equation (3.24) is used.

$$
\begin{aligned}
P_{t f, \text { loss }} & =\left(1-\eta_{t f}\right) P_{e, \text { out }}=P_{e, \text { out }}-P_{\text {trac }, \text { wheel }} \\
& =P_{e, \text { out }}-\left(F_{\text {aero }}+F_{r r}\right) V
\end{aligned}
$$

The engine overall efficiency and the power of fuel can be obtained with equation (3.16) and (3.17) respectively. The fuel rate and power loss in the engine for steady speed cases are also a little simpler than equations (3.18) and (3.19) of PnG case because of zero inertial force.

$$
\begin{aligned}
\dot{m}_{\text {fuel }}= & \frac{P_{\text {fuel }}}{L H V} \\
= & \frac{P_{e, \text { out }}}{\eta_{e} L H V}=\frac{P_{\text {trac, wheel }}}{\eta_{e} \eta_{t f} L H V}=\frac{1}{\eta_{e} \eta_{t f} L H V}\left(F_{\text {aero }}+F_{r r}\right) V \\
P_{e, \text { loss }} & =\left(1-\eta_{e}\right) P_{\text {fuel }}=P_{\text {fuel }}-P_{e, \text { out }} \\
& =P_{\text {fuel }}-\frac{1}{\eta_{\text {tf }}}\left(F_{\text {aero }}+F_{r r}\right) V
\end{aligned}
$$

\subsection{Determination of Parameters}

In the PnG driving strategy, there are a few important parameters that should be determined because they affect fuel consumption while the engine is operating. The important parameters are; speed range, acceleration time and speed difference between low and high speed limits of PnG drive cycles. Those parameters are directly connected to gear selection and engine torque which are dependent on each other because engine speed can be determined by an engaged gear at a certain vehicle speed. Therefore, it is assumed to use fixed gear (no gear shifting) at each PnG drive cycle to avoid complexity of analysis due to large engine efficiency changes. Note that there are no grade considerations in this study, but they could be easily included. More details are discussed in the following sections. 


\subsubsection{Acceleration Time}

In a conventional vehicle, higher vehicle acceleration requires higher engine load and it can obviously affect fuel consumption of PnG cases. Hence, different acceleration times are considered in this study for investigating the effect of higher engine load on fuel consumption. As shown in Figure 3.3, different acceleration time cases have same average vehicle speed and same vehicle speed profile for coast phase from same high vehicle speed. The average speed of the PnG drive cycles is used for comparison with steady speed. The vehicle miles traveled (VMT) of each PnG case varies due to different cycle times. Note that the basic speed difference between low and high speeds for PnG cycles is set to 10 miles per hour (mph) for example.

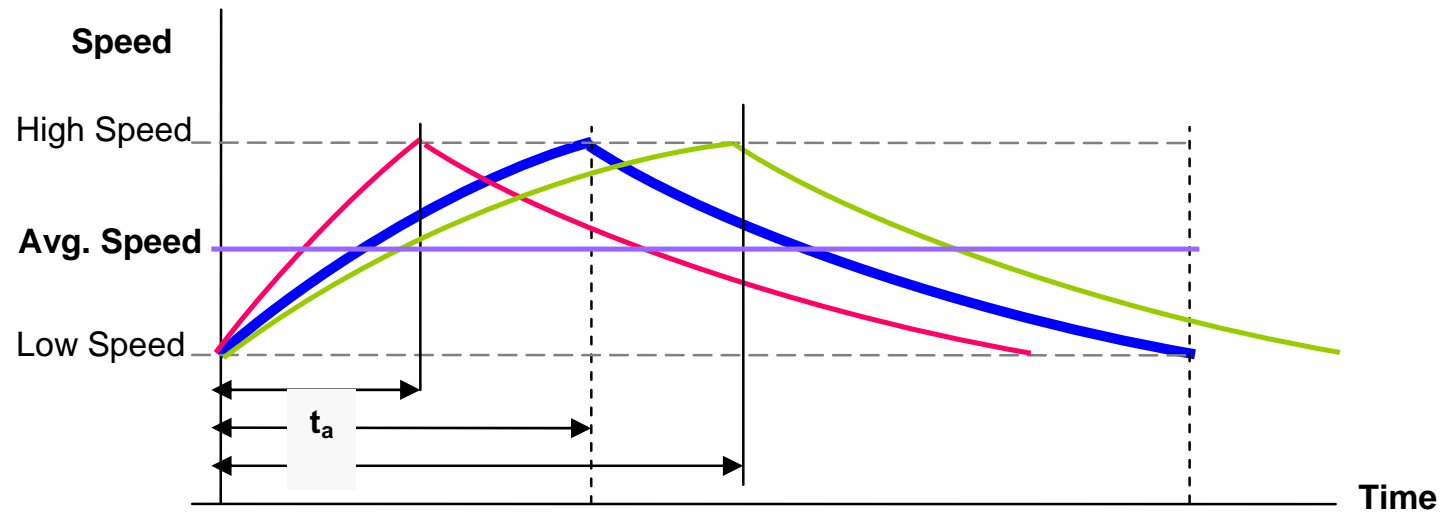

Figure 3.3 Determination of parameters: acceleration time

For the PnG cases with different acceleration times, the engine is operating in the same speed range because the gear is fixed and the vehicle speed range is same. However, engine torque increases or decreases as vehicle acceleration becomes higher or lower respectively as shown in Figure 3.4. If the engine is in a higher torque region, the engine overall efficiency can be increased. In contrast to that, lower engine torque means that the engine is operating in a low efficiency region. This engine load leveling obviously affects fuel consumption in a conventional vehicle. In this study, acceleration times between 10 and 30 seconds are considered. 


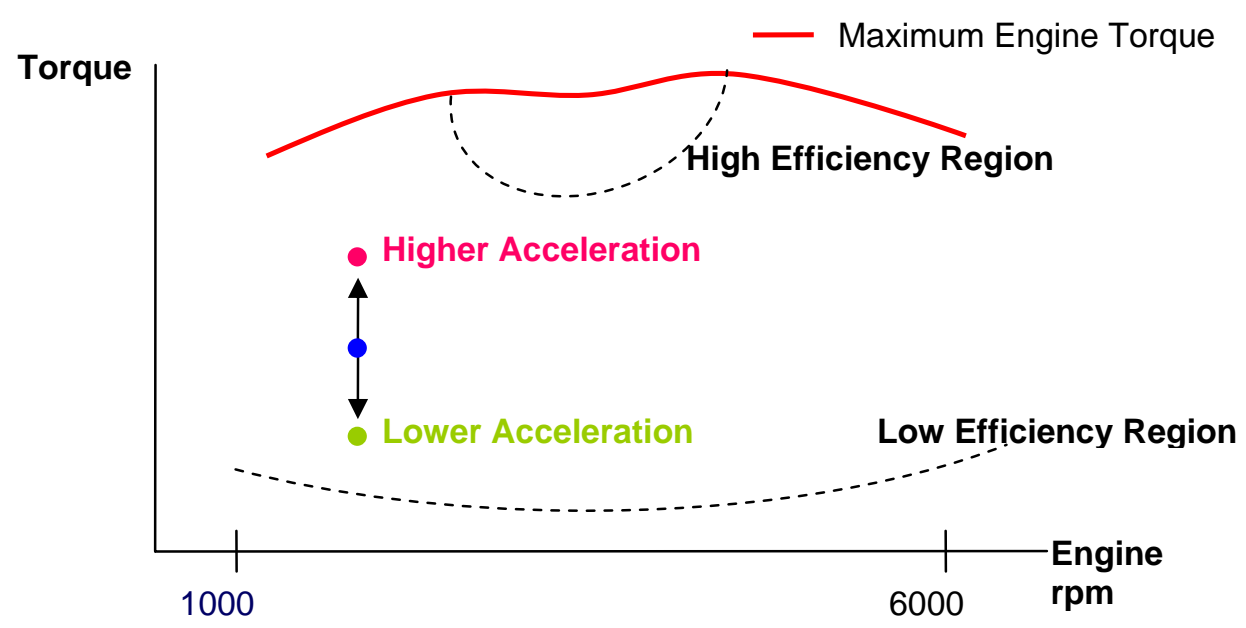

Figure 3.4 Engine operating region for different acceleration rates, fixed gear condition

\subsubsection{Speed Range}

The speed range is also one of the very important factors because the aerodynamic drag force is proportional to the square of vehicle speed as shown in equation (3.1), so it becomes more dominant at higher speed. Increased aerodynamic drag results in increase of engine load and more fuel consumption, but also higher engine efficiency for the steady speed case. Figure 3.5 shows different speed ranges of PnG cases with different average speeds. These cases show that the coast phase becomes longer as the vehicle speed range is lowered. Again, the VMT of each PnG case varies due to different speed ranges and cycle times.

For different vehicle speed ranges, the engine speed ranges of PnG cycles vary. In addition, the engine torque increases as vehicle speed range is increased because more tractive force is required by higher aero drag resistance. In contrast to that, the engine torque decreases as vehicle speed range is decreased. As shown in Figure 3.6, the engine is operating in a high efficiency region for the higher speed range, however high engine efficiency does not always mean lower fuel consumption for a given condition, so it is important to look at its impact on fuel consumption by PnG driving strategy. The investigated speed ranges are; 20 to $30 \mathrm{mph}, 30$ to $40 \mathrm{mph}$, and 40 to $50 \mathrm{mph}$ in this study. 


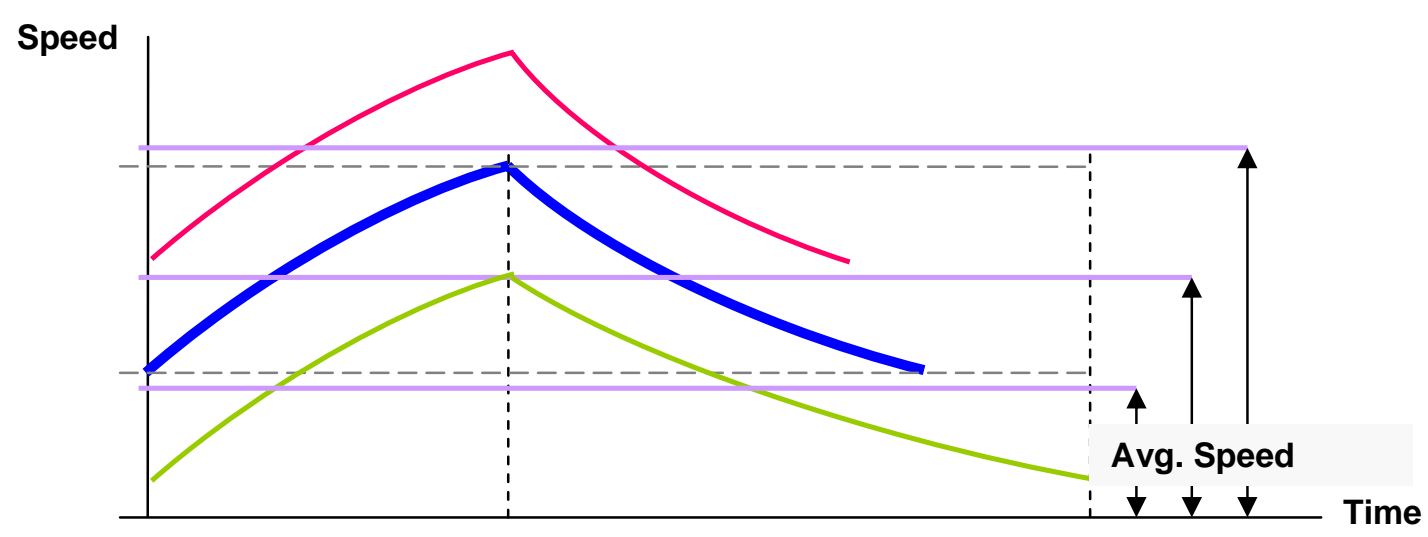

Figure 3.5 Determination of parameters: speed range

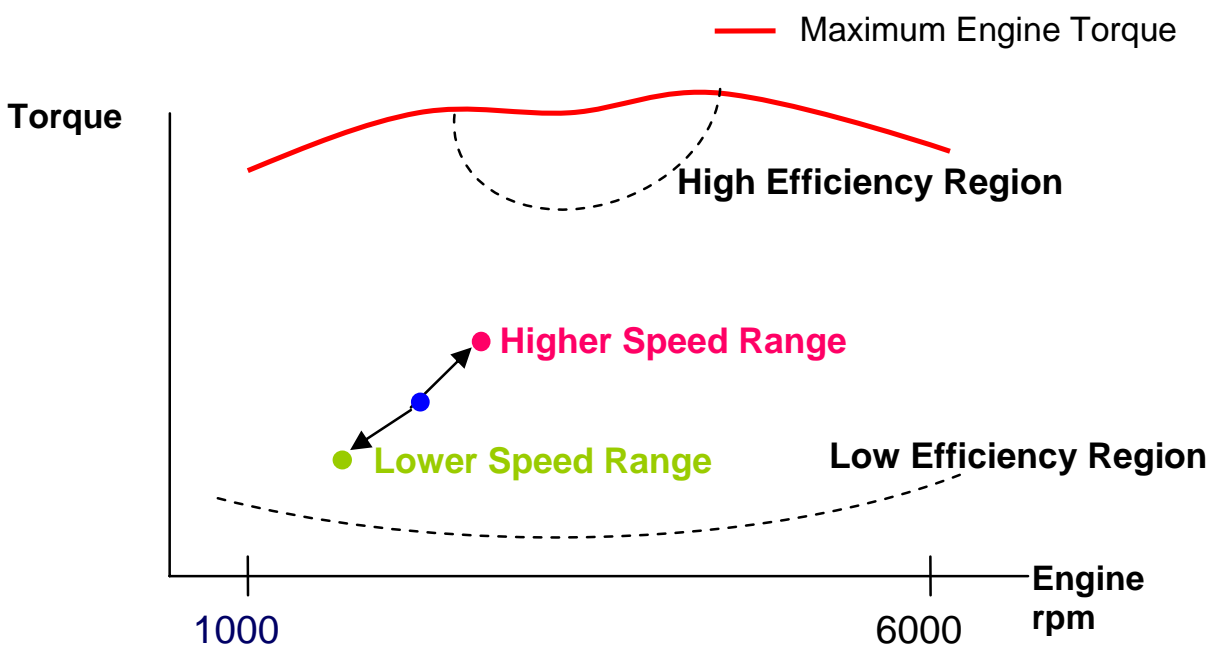

Figure 3.6 Engine operating region for different speed ranges, fixed gear condition

\subsubsection{Speed Difference}

The speed difference for each $\mathrm{PnG}$ case is $10 \mathrm{mph}$ as previously mentioned. However, larger speed difference can increase engine load and affect fuel consumption in a conventional vehicle. Hence, a larger speed difference $(20 \mathrm{mph})$ case is also considered to compare its impact to the PnG case with $10 \mathrm{mph}$ speed difference and the steady speed case. Both 10 and $20 \mathrm{mph}$ cases have the same average speed, different VMTs and different overall cycle times as shown in Figure 3.7. 
In a fixed gear condition, the engine speed and torque increase as the speed difference of PnG driving becomes larger than the basic case which has $10 \mathrm{mph}$ of speed difference. This case is shown in Figure 3.8. The smaller speed difference for example, $5 \mathrm{mph}$, is not considered in this parametric study because if the speed difference in PnG driving becomes smaller, then the fuel consumption would be closer to the steady speed case eventually. Also, the speed difference larger than $20 \mathrm{mph}$ is not considered because the gear should be shifted for that condition, so it can not only affect engine load and efficiency significantly but also make the problem more complex.

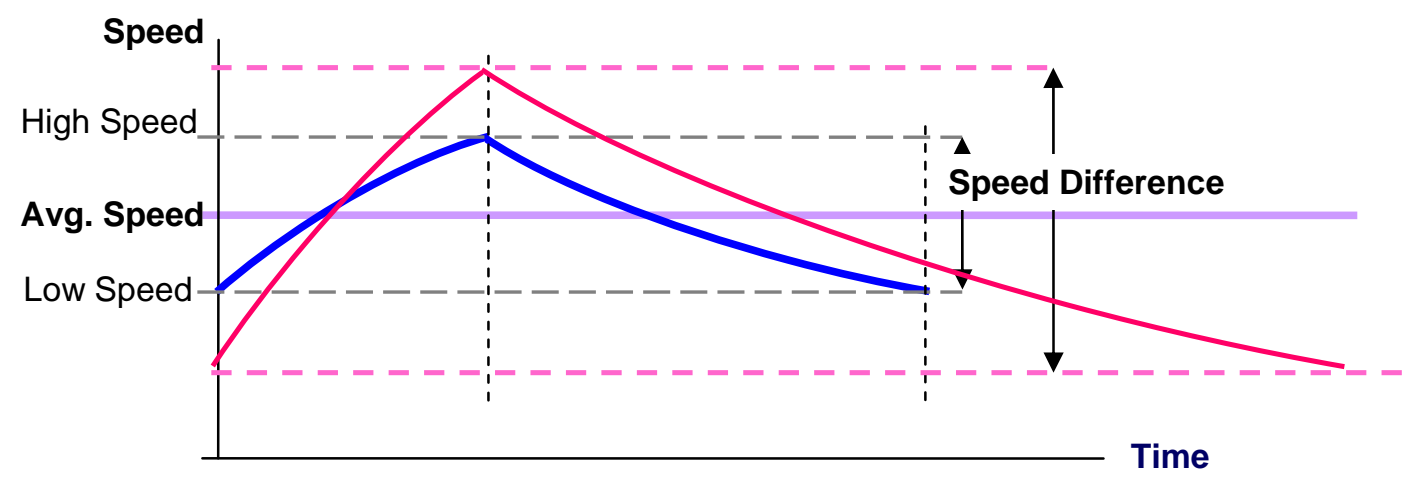

Figure 3.7 Determination of parameters: speed difference

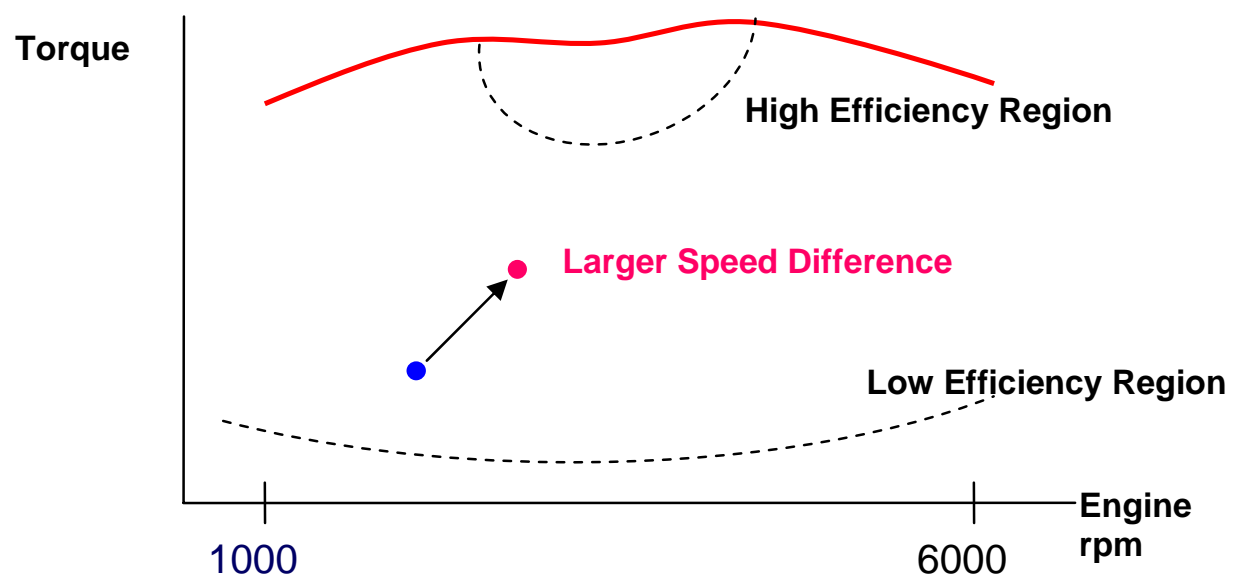

Figure 3.8 Engine operating for two speed differences, fixed gear condition 


\subsubsection{Deceleration Rate}

In contrast to the acceleration time introduced in section 3.3.1, the deceleration rate is considered as another parameter for the PnG driving. The basic assumption of the PnG driving strategy is that an engine should be off and a gear should be disengaged during coasting to maximize the benefit of storing $\mathrm{KE}$ in vehicle inertia. However, the deceleration rate obviously affects the VMT and it changes fuel economy because it is the fuel consumption divided by the VMT. Note that the deceleration rate is used instead of the deceleration time to easily apply the deceleration rate of coast down for a vehicle as a base case. Also, mechanical braking is not considered in this study because it is absolutely a frictional loss and a waste of KE. In order to investigate the effect of the deceleration rate, a simple engine compression braking model is made by few equations and estimation. The engine compression braking means that a gear and a clutch are engaged to an engine so the engine absorbs a certain amount of tractive power or wheel torque as the vehicle decelerates.

For deceleration with fuel cut off and engaged clutch/gear in engine compression braking, the engine produces negative torque. The brake mean effective pressure (bmep) for the negative torque is estimated by equation (3.27).

$$
\text { bmep }=-0.02 N_{e, r p m}-80(<0)
$$

Where: $N_{e, r p m}$ : Engine speed (rpm)

bmep : Brake mean effective pressure $(\mathrm{kPa})$

Note that equation (3.27) is developed for this work from some typical 4-cylinder, 2.0 L gasoline engine motoring data. The negative torque can be calculated using the bmep equation above.

$$
T_{e, \text { motoring }}=\frac{b m e p \times V_{d}}{4 \pi}=\frac{\left(-0.02 N_{e, r p m}-80\right) \times V_{d}}{4 \pi}(<0)
$$

Where: $T_{e, \text { motoring }}$ : Engine torque when engine is motoring (in engine compression braking) $(\mathrm{Nm})$

$V_{d}$ : engine displacement (L)

The negative torque can be simply converted to the negative power by equation (3.29)

$$
P_{e, \text { motoring }}=2 \pi N_{e} T_{e, \text { motoring }}(<0)
$$


Where: $P_{e, \text { motoring }}$ : Engine power when engine is motoring (in engine compression braking) (W)

$N_{e}:$ Engine speed (rev/s)

Substituting equations (3.28) and (3.29) to equation (3.9) gives equation (3.30) with respect to torque and equation (3.31) with respect to power respectively.

$$
\begin{aligned}
& \left(T_{e, \text { motoring }}-\frac{P_{a c c}}{2 \pi N_{e}}\right) \frac{N_{t f} \eta_{t f}}{r_{r}}=F_{\text {aero }}+F_{r r}+F_{I} \Rightarrow\left(T_{e, \text { motoring }}-\frac{P_{a c c}}{2 \pi N_{e}}\right)=\frac{r_{r}}{N_{t f} \eta_{t f}}\left(F_{a e r o}+F_{r r}+F_{I}\right) \\
& \left(P_{e, \text { motoring }}-P_{\text {acc }}\right) \eta_{t f}=\left(F_{\text {aero }}+F_{r r}+F_{I}\right) V \Rightarrow\left(P_{e, \text { motoring }}-P_{a c c}\right)=\frac{1}{\eta_{t f}}\left(F_{\text {aero }}+F_{r r}+F_{I}\right) V
\end{aligned}
$$

Equations (3.30) and (3.31) can be written in terms of acceleration in equation (3.32)

$$
\begin{aligned}
& a=\frac{1}{m_{I}}\left(\left(T_{e, \text { motoring }}-\frac{P_{a c c}}{2 \pi N_{e}}\right) \frac{N_{t f} \eta_{t f}}{r_{r}}-F_{\text {aero }}-F_{r r}\right) \text { or } \\
& a=\frac{1}{m_{I}}\left(\frac{\left(P_{e, \text { motoring }}-P_{a c c}\right) \eta_{t f}}{V}-F_{\text {aero }}-F_{r r}\right) \quad\left(\because F_{I}=m_{I} a\right)
\end{aligned}
$$

Note that the acceleration in equation (3.32) becomes negative in engine compression braking which means deceleration.

Using equation (3.32), the vehicle speed and deceleration rate of the conventional vehicle during engine compression braking from $60 \mathrm{mph}$ are calculated. The coast down case is calculated by equation (3.22). Figures 3.9 shows the vehicle speed when the vehicle is in coast down and in engine compression braking with different gears. The vehicle speed is slowly decreased in coast down but it is decreased much faster when the gear is engaged. When the gear is low, the vehicle speed decreases even faster due to a higher gear ratio. The deceleration rate is also shown in Figure 3.10 below. In coast down, the deceleration rate is about $0.20 \mathrm{~m} / \mathrm{s}^{2}$ at $80.5 \mathrm{~km} / \mathrm{h}$ $(50 \mathrm{mph})$ and $0.11 \mathrm{~m} / \mathrm{s}^{2}$ at $48.3 \mathrm{~km} / \mathrm{h}(30 \mathrm{mph})$ respectively. In contrast to that, the deceleration in engine compression braking with gear 5 is approximately $0.73 \mathrm{~m} / \mathrm{s}^{2}$ at $80.5 \mathrm{~km} / \mathrm{h}(50 \mathrm{mph})$ and $0.58 \mathrm{~m} / \mathrm{s}^{2}$ at $48.3 \mathrm{~km} / \mathrm{h}(30 \mathrm{mph})$ respectively because then engine absorbs power or torque 
through the driveline and it operates like mechanical braking. For lower gears, the deceleration rates are even much higher than gear 5.

Vehcle Speed Comparison during Deceleration for 2007 Ford Focus from Simple Vehicle Model

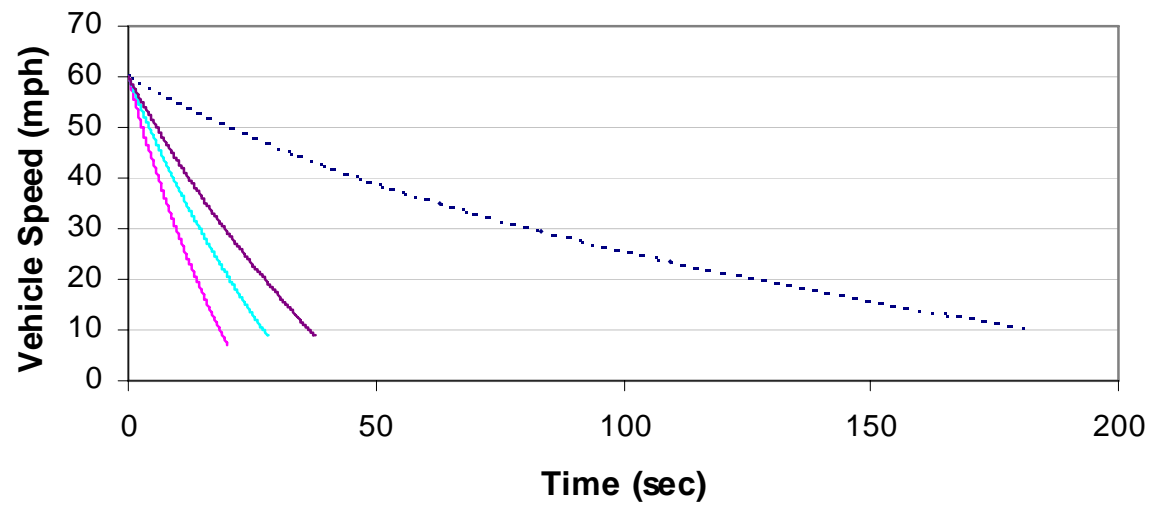

...... Coast Down — Gear 3 — Gear $4-$ Gear 5

Figure 3.9 Vehicle speed comparison between coast down and engine compression braking for a conventional vehicle, 2007 Ford Focus

\section{Deceleration Rate Comparison during Deceleration for 2007 Ford Focus from Simple Vehicle Model}

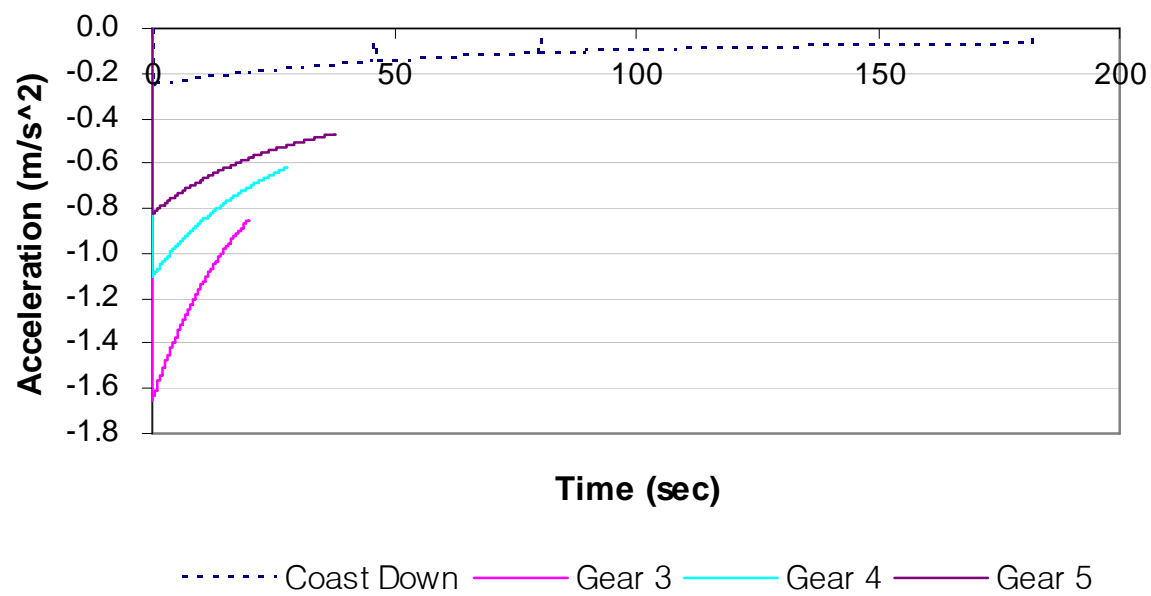

Figure 3.10 Deceleration rate comparison between coast down and engine compression braking for a conventional vehicle, 2007 Ford Focus

The higher deceleration rate in engine compression braking decreases the vehicle speed much faster than in coast down which results in shorter distance from engine friction losses. Thus, the 
case of engine compression braking is not considered for a conventional vehicle study. However, slower or faster deceleration rates possibly affect fuel consumption in HEVs because they have a regenerative brake system to recover energy during deceleration without mechanical braking or engine operation. Therefore, it is still open to study the effect of deceleration rate in the PnG driving strategy for HEVs. More details are discussed in Chapters 5 and 6.

\subsection{Efficiency of Kinetic Energy Storage and Net Benefit of PnG}

In this section, a new term; 'efficiency of KE storage,' is defined to look at the impact of PnG driving closely in comparison with that of steady speed driving. In addition, the net benefit of $\mathrm{PnG}$ is described with respect to fuel energy ratios of both cases.

\subsubsection{Efficiency of Kinetic Energy Storage}

Let's assume that the acceleration (pulse) phase is from point 1 to point 2 and the coast (glide) phase is from point 2 to point 3 as demonstrated in Figure 3.11. While accelerating a vehicle, more fuel energy is required in contrast with steady speed case. This additional fuel energy is transferred to KE and storage after losses in engine and driveline. The stored $\mathrm{KE}$ in vehicle inertia can be discharged later in coasting to overcome rolling resistance and aero drag forces.

Now, the efficiency of KE storage can be defined as

$$
\eta_{\text {inertia }}=\frac{E_{i, P n G, 1 \rightarrow 2}}{E_{\text {fuel, } P n G, 1 \rightarrow 2}-E_{\text {fuel,cruising }, 1 \rightarrow 2}}
$$

where: $\eta_{\text {inertia }}$ : Efficiency of KE storage during acceleration

$\boldsymbol{E}_{\boldsymbol{i}, P n G, 1 \rightarrow 2}$ : Energy stored in vehicle inertia during acceleration $(\mathrm{kJ})$

$E_{f u e l, P n G, 1 \rightarrow 2}$ : Fuel energy of PnG case during acceleration $(\mathrm{kJ})$

$E_{\text {fuel,cruising, } 1 \rightarrow 2}$ : Fuel energy of steady speed case during the same time interval of acceleration in $\mathrm{PnG}$ case $(\mathrm{kJ})$

The denominator in equation (3.33) represents the difference of fuel energy between PnG and steady speed cases during acceleration interval of the PnG case. In other words, it means the 
additional fuel energy due to vehicle acceleration in the PnG case compared to the steady speed case. Therefore, $\eta_{\text {inertia }}$ is defined as the KE stored in vehicle inertia during acceleration divided by the additional fuel energy due to vehicle acceleration.

(a) Pulse and Glide

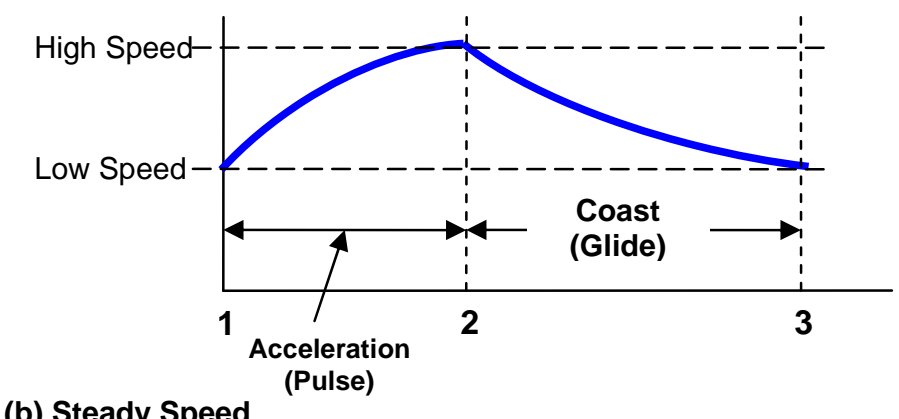

(b) Steady Speed

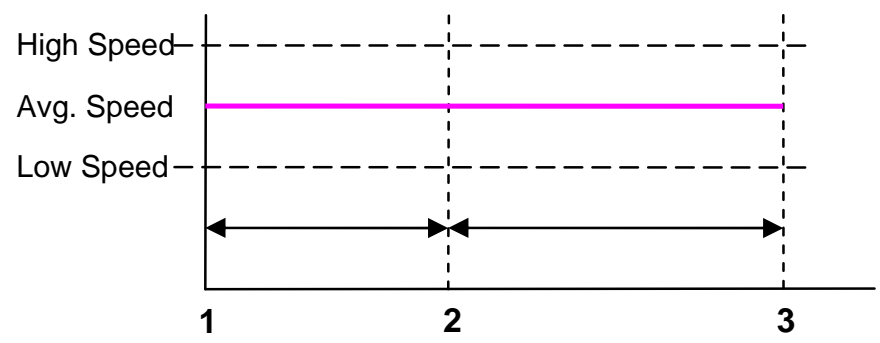

Figure 3.11 PnG and steady speed cases with two separated intervals

\subsubsection{Net Benefit of PnG}

The stored $\mathrm{KE}$ in vehicle inertia during acceleration can be discharged during coasting. In true coasting of PnG driving, the engine is turned off so there is no fuel use $\left(E_{\text {fuel, } P n G, 2 \rightarrow 3}=0\right)$. However, the engine still consumes fuel to overcome road load in steady speed driving for the same time interval of coasting in PnG (from 2 to 3). The fuel ratio of steady speed case to discharging energy stored in vehicle inertia during coasting can be described as shown below.

$$
\begin{aligned}
F R_{2 \rightarrow 3}= & \frac{E_{f u e l, \text { crui sin } g, 2 \rightarrow 3}}{\frac{E_{i, P n G, 1 \rightarrow 2}}{\eta_{\text {inertia }}}+E_{f u e l, P n G, 2 \rightarrow 3}} \\
= & \frac{E_{\text {fuel, cruisin } g, 2 \rightarrow 3}}{E_{\text {fuel, } P n G, 1 \rightarrow 2}-E_{f u e l, \text { cruising }, 1 \rightarrow 2}}
\end{aligned}
$$


where: $F R_{2 \rightarrow 3}$ : Fuel ratio of steady speed case to $\mathrm{PnG}$ case while discharging energy stored in vehicle inertia during coasting

$E_{\text {fuel,cruising,2 } \rightarrow 3}$ : Fuel energy of steady speed case during the same time interval of coasting in PnG case $(\mathrm{kJ})$

$E_{\text {fuel,PnG,2 } \rightarrow 3}$ : Fuel energy of PnG case during coasting ( $=0$ in true coasting) $(\mathrm{kJ})$

The net benefit of PnG case to steady speed case can be carried out in terms of fuel ratio from equations discussed previously by combining two different phases for both cases; $1 \rightarrow 2$ and 2 $\rightarrow 3$ phases.

$$
\begin{aligned}
F R_{\text {PnG benefit }} & =\frac{E_{\text {fuel, cruisin } g, 1 \rightarrow 2}+E_{\text {fuel, }, \text { rrui sin } g, 2 \rightarrow 3}}{E_{\text {fuel, } P n G, 1 \rightarrow 2}+E_{f u e l, P n G, 2 \rightarrow 3}} \\
& =\frac{E_{\text {fuel, cruisin } g, 1 \rightarrow 3}}{E_{\text {fuel, }, P n G, 1 \rightarrow 2}}
\end{aligned}
$$

where: $F R_{\text {PnG benefit }}$ : Fuel ratio of steady speed case to PnG case for the time of one complete PnG drive cycle - net benefit of PnG

According to results of a conventional vehicle in the following section, the fuel ratio in equation (3.29) turns out to be very similar to the inverse of the fuel economy ratio of a PnG case to a steady speed case as shown below.

$$
F R_{\text {PnG benefit }}=\frac{E_{\text {fuel,crui sin } g, 1 \rightarrow 3}}{E_{\text {fuel }, P n G, 1 \rightarrow 2}} \cong \frac{F E_{P n G}}{F E_{\text {cruising } g}}
$$

\subsection{Results for a Conventional Vehicle}

The results of PnG and steady speed cases for a conventional vehicle are described in this section. The conventional vehicle simulated in the simple vehicle model is 2007 Ford Focus with $2.3 \mathrm{~L}$ engine and 5-speed manual transmission. It is a small size sedan (compact car) and its size is relatively similar to 2004 Toyota Prius which is selected as an HEV case in this study. The vehicle is simulated for one complete PnG cycle and same period of steady speed cycle so each PnG and steady speed case shows same overall cycle time and VMT as shown in Tables B.2, B.3 
and B.4 in Appendix B. Also the average speeds of each PnG case are very similar to $25 \mathrm{mph}$, $35 \mathrm{mph}$ and $45 \mathrm{mph}$ respectively. The following sections demonstrate more details.

\subsubsection{Vehicle Specifications}

As briefly described above, a 2007 Ford Focus is used to simulate PnG and steady speed cases in the simple vehicle model. The vehicle specifications such as vehicle mass, frontal area, drag coefficient and rolling resistance coefficient are tabulated in Table 3.1 [50].

Table 3.1 Vehicle specifications of 2007 Ford Focus used in the simple vehicle model

\begin{tabular}{|l|c||l|c|}
\hline Vehicle Model & Ford Focus & Transmission & 5-speed manual \\
\hline Model Year & 2007 & Frontal Area $\left(\mathrm{m}^{2}\right)$ & 2.06 \\
\hline Vehicle Mass $(\mathrm{kg})$ & $\begin{array}{c}1417.5 \\
(3125 \mathrm{lb})\end{array}$ & Drag Coefficient & 0.31 \\
\hline Engine & 2.3 L gasoline & $\begin{array}{l}\text { Rolling Resistance } \\
\text { Coefficient }\end{array}$ & 0.007 \\
\hline
\end{tabular}

\subsubsection{Fuel Consumption}

Figure 3.12 shows a contour map of fuel economy results of PnG in different speed ranges and acceleration times from the simple simulation model for 2007 Ford Focus. In this simulation, the higher gear is used if the engine can generate enough torque to accelerate the vehicle because higher gear makes engine running at low speed/high torque, and it is generally beneficial for fuel economy. However, different gears are used for each speed range due to engine lug limit $(\sim 1300$ rpm); gear 3 for $20-30 \mathrm{mph}$, gear 4 for $30-40 \mathrm{mph}$, and gear 5 for $40-50 \mathrm{mph}$ respectively. As previously described in section 3.2, the fuel economy results shown in Figure 3.12 is generated from different values of constant engine torque for each PnG case. 


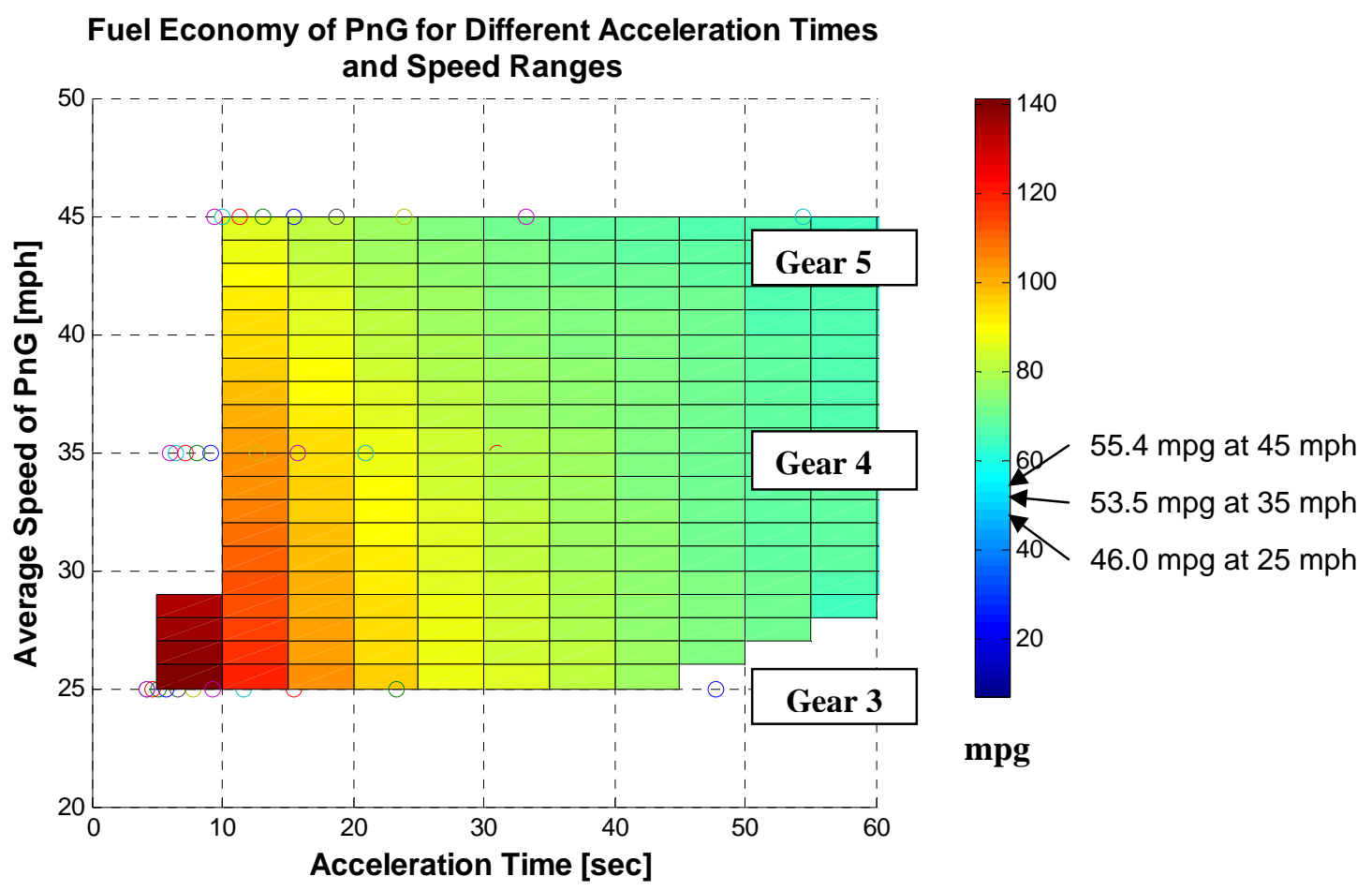

Figure 3.12 Contour map of fuel economy results of PnG from the simple vehicle model along with different acceleration times and speed ranges, 2007 Ford Focus

In Figure 3.12, the fuel economy of PnG increases as the acceleration times becomes shorter and the speed range becomes lower. The fuel economy is improved until the vehicle can achieve the shortest acceleration time (highest engine torque) for each speed range in valid engine overall efficiency region. The shortest acceleration time and fuel economy at three different speed ranges is summarized in Table 3.2 below. More data is shown in Table B.1 in Appendix B.

Table 3.2 Shortest acceleration time and fuel economy of PnG that the vehicle can achieve in the simple vehicle model (2007 Ford Focus)

\begin{tabular}{|c|c|c|c|}
\hline $\begin{array}{c}\text { Speed Range of PnG } \\
(\mathrm{mph})\end{array}$ & $\begin{array}{c}\text { Acceleration Time } \\
(\mathrm{sec})\end{array}$ & $\begin{array}{c}\text { Fuel Economy } \\
(\mathrm{mpg})\end{array}$ & Gear \\
\hline $20-30$ & 4.2 & 146.9 & 3 \\
\hline $30-40$ & 6.1 & 112.7 & 4 \\
\hline $40-50$ & 9.4 & 85.3 & 5 \\
\hline
\end{tabular}

From the fuel economy results of PnG plotted in Figure 3.13, several cases are selected for three different speed ranges to compare the results to the steady speed cases as listed in Table 3.3. For steady speed cases, the average speed for each PnG speed range is used. The 5 seconds of 
acceleration time for $30-40$ and $40-50 \mathrm{mph}$ PnG cases cannot be simulated due to engine torque limit (see Table 3.2) in the simple vehicle model.

Table 3.3 Selected cases to compare the fuel economy improvement of $\mathrm{PnG}$ and steady speed driving strategies in the simple vehicle model

\begin{tabular}{|c||c|c|c|c|c|c|c||c|}
\hline \multirow{2}{*}{$\begin{array}{c}\text { Steady Speed } \\
(\mathrm{mph})\end{array}$} & \multicolumn{6}{|c||}{ PnG } & \multirow{2}{*}{ Gear } \\
\cline { 2 - 10 } & Speed Range (mph) & \multicolumn{5}{|c|}{ Acceleration Time (sec) } & \\
\hline 25 & $20-30$ & 5 & 10 & 15 & 20 & 25 & 30 & 3 \\
\hline 35 & $30-40$ & N/A & 10 & 15 & 20 & 25 & 30 & 4 \\
\hline 45 & $40-50$ & N/A & 10 & 15 & 20 & 25 & 30 & 5 \\
\hline
\end{tabular}

Fuel Economy Comparison for Different Acceleration Time 2007 Ford Focus

(Constant engine torque at each case)

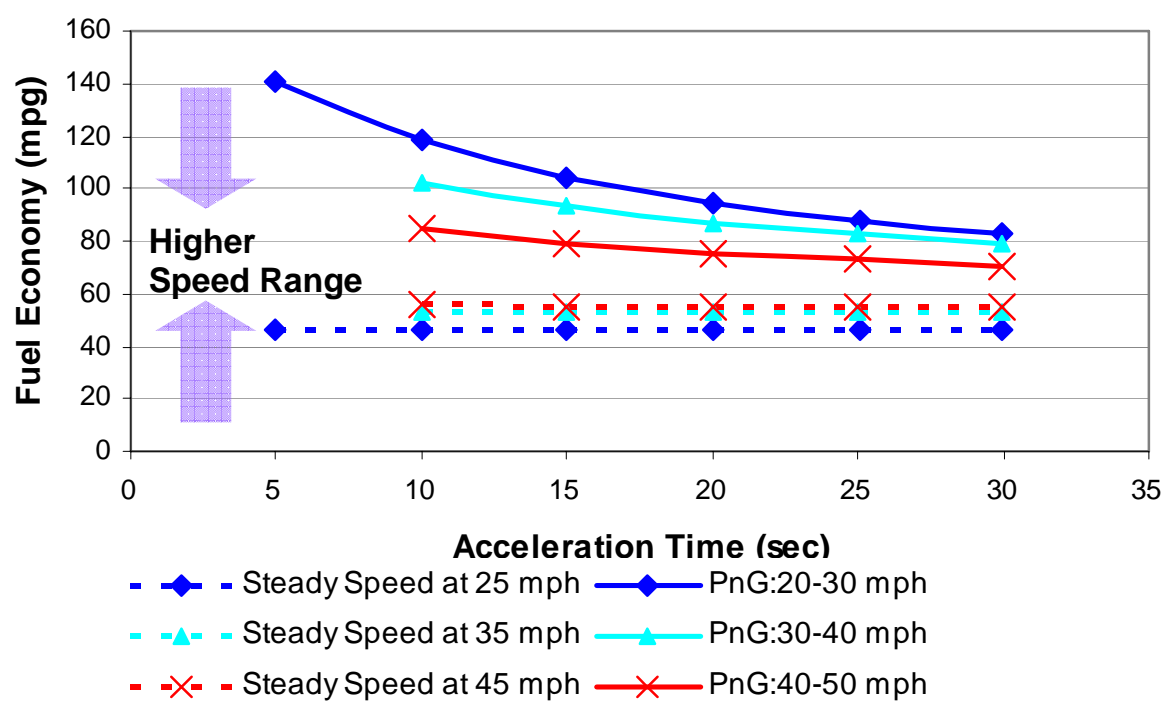

Figure 3.13 Fuel economy results from simple vehicle model in different speed ranges and acceleration times, 2007 Ford Focus

In Figure 3.14, the fuel economy results of the PnG driving case between 25 and $45 \mathrm{mph}$ with larger speed difference $(20 \mathrm{mph})$ and different acceleration times are shown. The fuel economy, in gray line, for $10 \mathrm{mph}$ of speed difference is plotted as well to be compared. The average speed of $20 \mathrm{mph}$ of speed difference should be the same as that of $10 \mathrm{mph}$ of speed difference. Hence the fuel economy of steady speed case at average speed, $35 \mathrm{mph}$, in Figure 3.14 is the same as that in Figure 3.13. Note that different gears are used for each case; gear 4 for PnG with $10 \mathrm{mph}$ of speed difference, gear 3 for PnG with $20 \mathrm{mph}$ of speed difference, and gear 4 for 
steady speed respectively. For the larger speed difference case, engine cannot generate enough torque to propel the vehicle in gear 4 .
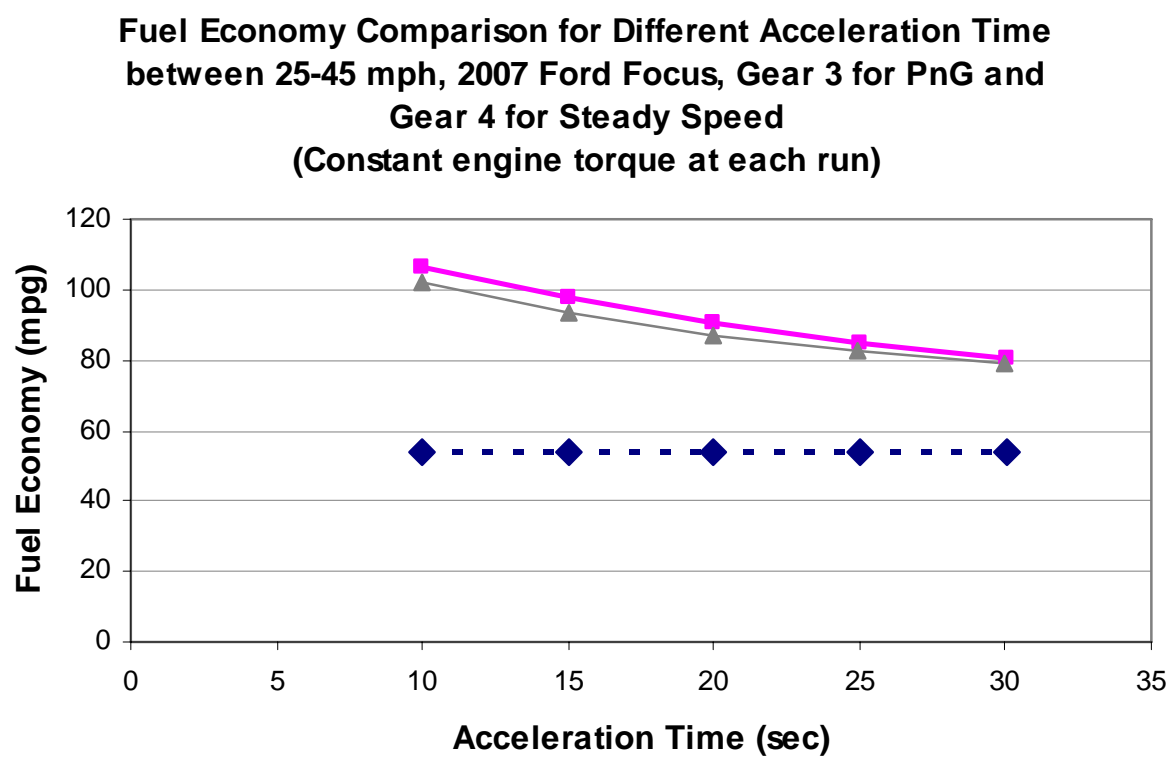

- $\downarrow$ - Steady Speed at $35 \mathrm{mph} \longrightarrow$ PnG: $25-45 \mathrm{mph} \longrightarrow$ PnG: $30-40 \mathrm{mph}$

Figure 3.14 Fuel economy results from simple vehicle model between $25 \mathrm{mph}$ and $45 \mathrm{mph}$ in different acceleration times, 2007 Ford Focus

The fuel economy of PnG with the larger speed difference is slightly higher than that of PnG with $10 \mathrm{mph}$ of speed difference because engine load is higher due to higher acceleration and engine overall efficiency is increased. However, the improvement of fuel economy is relatively smaller for the larger speed difference and it possibly needs gear shifting. Therefore, $10 \mathrm{mph}$ of speed difference will only be considered for the rest of this study. Note that larger speed difference cases for lower speed ( $35 \mathrm{mph}$ to $55 \mathrm{mph}$ ) and higher speed (15 mph to $35 \mathrm{mph}$ ) are not presented because both cases show gear shifting during acceleration.

\subsubsection{Efficiency of Kinetic Energy Storage}

The efficiency of KE storied in vehicle inertia, $\eta_{\text {inertia }}$, was introduced and defined in section 3.4. From the simulation in the simple vehicle model, $\eta_{\text {inertia }}$ for all PnG cases is also calculated and its data is plotted in Figure 3.15. 


\section{Efficiency of Kinetic Energy Storage during Acceleration \\ in PnG}

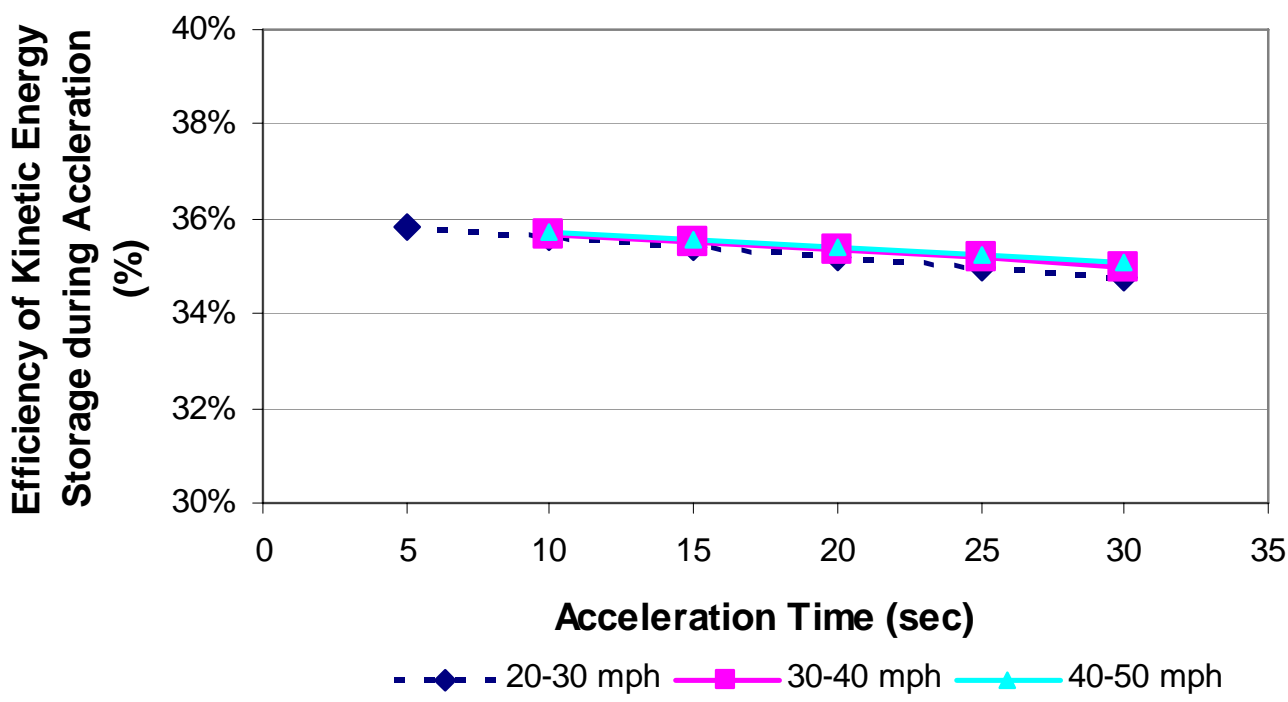

Figure 3.15 Efficiency of KE storage during acceleration in PnG, 2007 Ford Focus

The interesting point from this calculation is that $\eta_{\text {inertia }}$ remains almost constant for all cases within the range from $35.8 \%$ to $34.8 \%$ (it decreases slightly $(\sim 1 \%)$ as acceleration time increases). It means that the $\eta_{\text {inertia }}$ is always higher than engine overall efficiency; $18.1 \%$ $31.8 \%$ (see Tables B.2, B.3 and B.4 in Appendix B), during acceleration for all PnG cases and storing KE for coast phases is even more efficient than engine overall efficiency in steady speed driving conditions. Note that the $\eta_{\text {inertia }}$ is not an absolute engine efficiency but a ratio of an increase of inertial energy to an increase of fuel energy by engine load leveling during acceleration. In other words, increasing engine load with more fuel consumption during acceleration is more efficient because the stored $\mathrm{KE}$ by additional fuel energy will be used in coast phase without further energy loss. In conclusion, the preliminary study demonstrated in this chapter shows that the PnG driving strategy shows some positive potential to reduce fuel consumption compared to steady speed driving and merits for further investigation. 


\section{Chapter 4 In-Depth Vehicle Simulation for Conventional and Hybrid Electric Vehicles using Powertrain System Analysis Toolkit (PSAT)}

After the preliminary study from the simple vehicle model, Powertrain System Analysis Toolkit (PSAT) version 6.2 [50] is used again to simulate impact of KE stored in vehicle inertia on fuel consumption by PnG driving strategy. The PSAT is a sophisticated vehicle simulation model developed by ANL, so it can generate more accurate and realistic results than the simple vehicle model described in Chapter 3. Also, the PSAT model includes accessory loads and more loss terms than the simple vehicle model. The vehicles simulated by PSAT are a conventional vehicle, 2007 Ford Focus, and an HEV, 2004 Toyota Prius. In order to simulate the vehicles, the drive cycles of PnG driving cases are generated from the simple vehicle model and with PSAT. This chapter demonstrates how the simulation has been performed for both conventional and hybrid electric vehicles, and discusses the simulation results from PSAT.

\subsection{Simulation of PnG and Steady Speed using PSAT}

As mentioned above, PSAT, developed by ANL is mainly used to simulate conventional and hybrid electric vehicles because it has many predefined configurations to simulate sophisticated and various types of vehicle systems and it is well validated to estimate fuel economy and 
performance [51]. PSAT is a forward looking model, so a drive cycle (vehicle speed versus speed profile) is required to give power/torque demand to a virtual driver. Note that a forward looking model analyzes the energy flow from powertrain to road with feed back control but a backward tracking model analyzes the energy flow from the road to powertrain. Also, PSAT can only launch a vehicle at zero speed in simulation, so a launch part is added necessarily in the drive cycles. The following sections discuss how to generate the drive cycles and how to analyze the data from simulation by PSAT.

\subsubsection{Generating PnG Drive Cycles}

The drive cycles for various PnG cases are made before simulating the conventional and hybrid electric vehicles. The PnG drive cycles used in PSAT consist of three parts; a launch part, an acceleration phase and a coast phase. The launch part is generated from the simple vehicle model to launch the simulated vehicle at zero speed. The vehicle is accelerated with a constant acceleration and then it maintains a particular speed for 10 seconds in each PnG drive cycle as shown in Figure 4.1. The 10 seconds of constant speed interval is added on purpose to distinguish it from the PnG part which will be solely used for data analysis.

The acceleration phase is generated from the simple vehicle model as well. It is assumed that the vehicle is being accelerated based on a constant power at driven wheels for each PnG case rather than a constant acceleration rate because it is much more similar to a constant power / torque demand at an accelerator (or "gas") pedal and makes it easier for a driver to follow the drive cycle in vehicle testing. The coast phase is generated by the built-in simulation of coast down in PSAT because the vehicle speed profile from the simple vehicle model is slightly different and it can either cause unnecessary fuel consumption if engine is on to follow it or speed trace miss if engine is off during coasting. The generated parts of the drive cycles for each case are combined together to make one complete PnG drive cycle as described in Figure 4.1.

For steady speed driving cases, only a launch part from the simple vehicle model is attached to a constant average speed of each PnG speed range for simulating a vehicle in PSAT. 


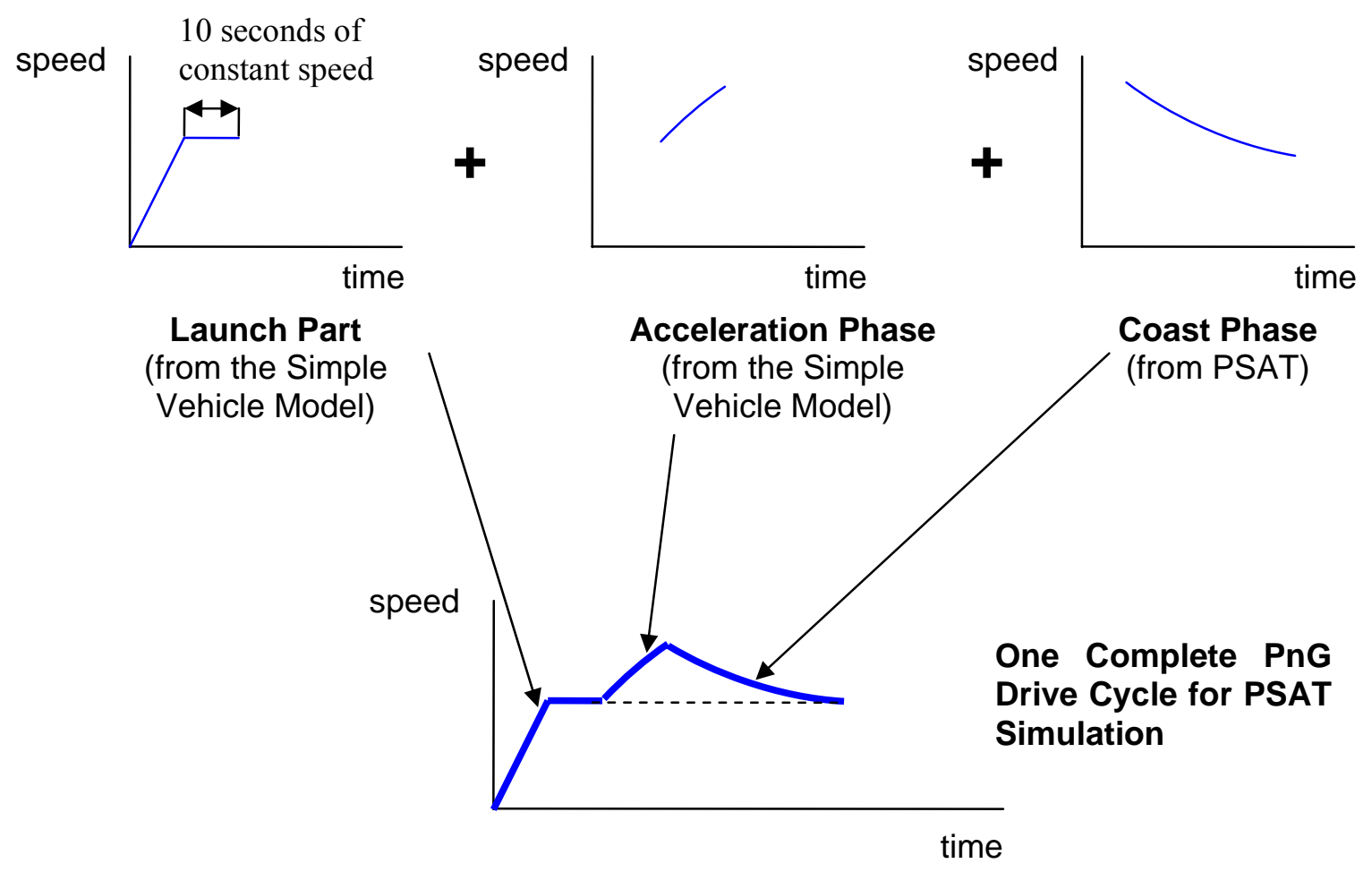

Figure 4.1 Generating a PnG drive cycle

\subsubsection{Method of Data Extraction from Simulation Results}

After a vehicle is simulated for a PnG case in PSAT, the launch part which is added to the drive cycles for running simulation is excluded to solely compare the PnG and steady speed intervals. Therefore, all data in the launch part such as cycle time, vehicle speed, distance, engine speed, fuel rate and battery SOC (only for an HEV) are excluded in analysis. This process is also applied to analyze data in an HEV vehicle testing in Chapter 6. 


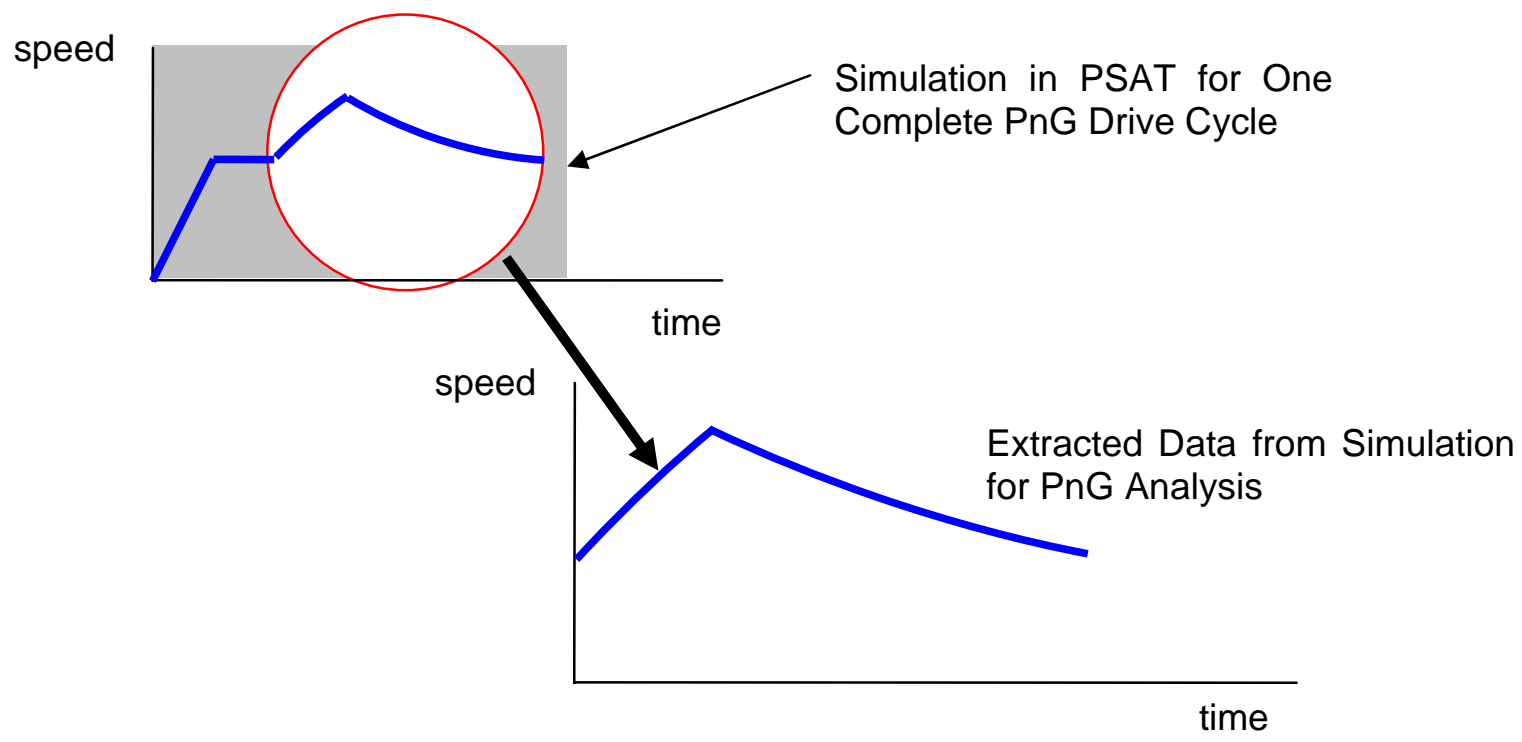

Figure 4.2 Method of data analysis from PSAT simulation results

\subsubsection{SOC Balance for an HEV in Simulation}

When analyzing fuel consumption of HEVs, one more important parameter that should be considered is the state of charge (SOC) of a battery because it has one more power source. The SOC of a battery varies because the HEV control strategy discharges/charges the electrical power from/to the battery depending on the vehicle power demand. As previously described in section 2.6, SAE recommends to balance an initial and a final SOC of a battery in HEVs to obtain accurate and reasonable fuel consumption data [40]. Therefore, in PSAT simulation, the SOC for each PnG cases is carefully considered for repeated PnG and steady speed drive cycles. In contrast to the SOC correction method for vehicle testing introduced in section 2.6, a different approach is used for PSAT simulation. 


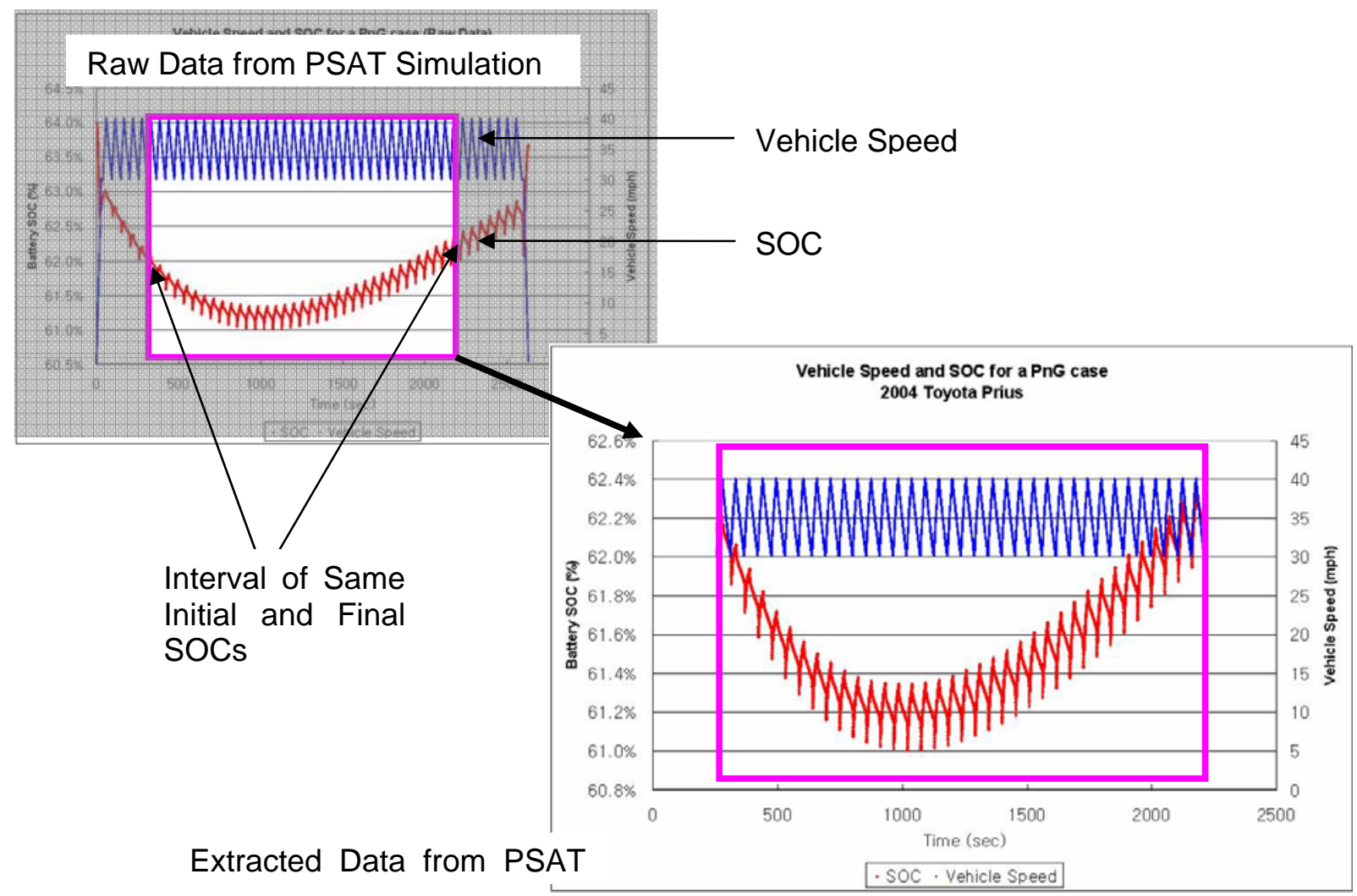

Figure 4.3 Example of data extraction for SOC balance from an HEV simulation

As shown in Figure 4.3, the SOC of the battery in PnG and steady speed drive cycles typically shows a swing over a cycle in simulation. Thus the interval that has same initial and final SOCs can be selected and extracted for data analysis.

\subsubsection{Selection of PnG Cases for In-Depth Simulation}

The in-depth simulation cases using PSAT are determined based on the simple vehicle model results. As discussed in Chapter 3, three different speed ranges; $20-30 \mathrm{mph}, 30-40 \mathrm{mph}$ and $40-50 \mathrm{mph}$, and six different acceleration times from 5 to 30 seconds with 5 second interval are selected for PnG cases. They are illustrated in Figure 4.4 below. 


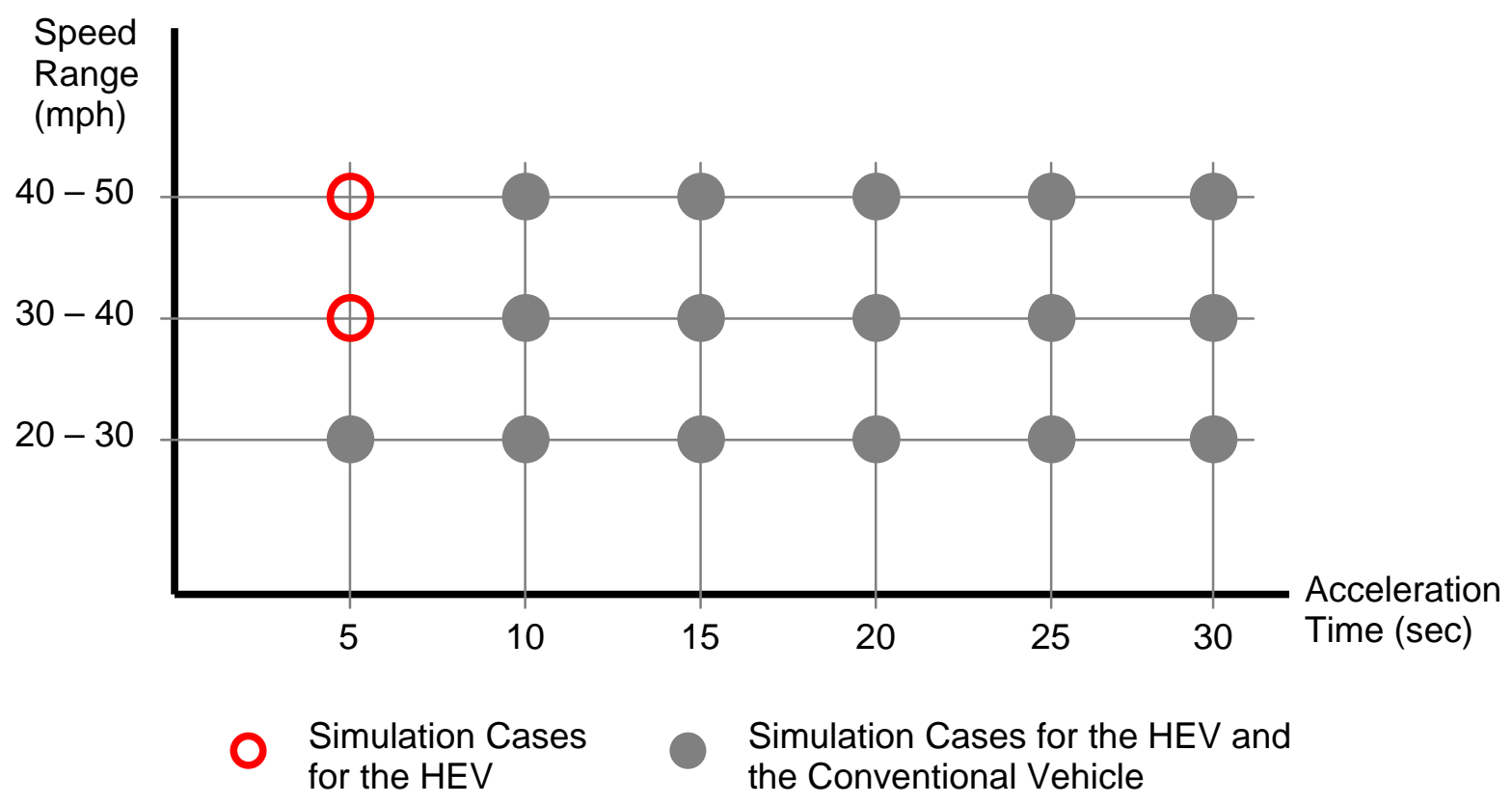

Figure 4.4 Selected PnG cases for in-depth simulation using PSAT for the conventional vehicle and the HEV

For the HEV, all 18 different PnG cases are selected for the in-depth simulation. However, two PnG cases; $30-40 \mathrm{mph}$ and 40 - $50 \mathrm{mph}$ with 5 seconds of acceleration are excluded for the conventional vehicle because the vehicle cannot achieve those conditions in a fixed gear condition due to engine torque limit as shown in the simple vehicle model results. Note that the fixed gear condition for each speed range is assumed in this study to avoid complexity of analysis by gear shifting. However, if gear shifting is allowed on those two conditions, the vehicle can definitely follow the vehicle speed trace. Also three different steady speeds; $25 \mathrm{mph}$, $35 \mathrm{mph}$ and $45 \mathrm{mph}$ are selected for in-depth simulation using PSAT for both conventional and hybrid electric vehicles.

\subsection{Conventional Vehicle: 2007 Ford Focus}

A conventional vehicle, 2007 Ford Focus, which is used in the preliminary study, is simulated in PSAT to compare with the results from the simple vehicle model discussed in Chapter 3 . The simulated cases are identical to the cases tabulated in Table 3.3. Again, the engine is off and the 
gear position is in $\mathrm{N}$ to avoid engine compression braking in the simulation. The drive cycles are generated with those conditions from the simple vehicle model and with PSAT.

\subsubsection{Vehicle Specifications}

Table 4.1 shows the vehicle specifications of 2007 Ford Focus. They are a little different from Table 3.1 because road load coefficients, A, B and C are used in PSAT simulation rather than frontal area, drag coefficient and rolling resistance. Note that the rolling resistance coefficient in Table 3.1 is lower, so that the road load of the vehicle in the simple vehicle model is lower than that of the PSAT vehicle model which uses the coefficients in Table 4.1. The road load coefficients tabulated in Table 4.1 are obtained from annual certification test results and data in Environmental Protection Agency (EPA) [52]. Note that the units of those coefficients are converted from English units in EPA data to SI units for use in PSAT.

Table 4.1 Vehicle specifications of 2007 Ford Focus used in PSAT

\begin{tabular}{|l|c||l|c|}
\hline Vehicle Model & Ford Focus & Transmission & 5-speed manual \\
\hline Model Year & 2007 & A $(\mathrm{N})$ & 111.61 \\
\hline Vehicle Test Mass $(\mathrm{kg})$ & 1417.5 & B $(\mathrm{N} /(\mathrm{m} / \mathrm{s}))$ & 6.68 \\
\hline Engine & 2.3 L gasoline & C $\left(\mathrm{N} /(\mathrm{m} / \mathrm{s})^{2}\right)$ & 0.31 \\
\hline
\end{tabular}

\subsubsection{Fuel Rate and Cumulative Fuel Use}

In a conventional vehicle, the only power source to produce power/torque to propel the vehicle is the engine so it is relatively easy to investigate the impact of the PnG driving strategy on fuel consumption. In Figure 4.5, the fuel rate for a PnG and a steady speed driving cases are plotted. The two dashed lines are vehicle speed and the others are fuel rate for both cases. In the plot, the steady speed driving shows constant speed at $25 \mathrm{mph}$ and constant fuel rate around $0.43 \mathrm{~g} / \mathrm{s}$. However, the vehicle consumes much more fuel during acceleration in the PnG driving than the steady speed driving. In coast phase, the vehicle does not consume fuel at all because the engine is off as shown in Figure 4.5. 
Fuel Rate Comparison between Steady Speed at $25 \mathrm{mph}$ and

PnG in 20 - 30 mph w/ 10 Seconds of Acceleration

2007 Ford Focus

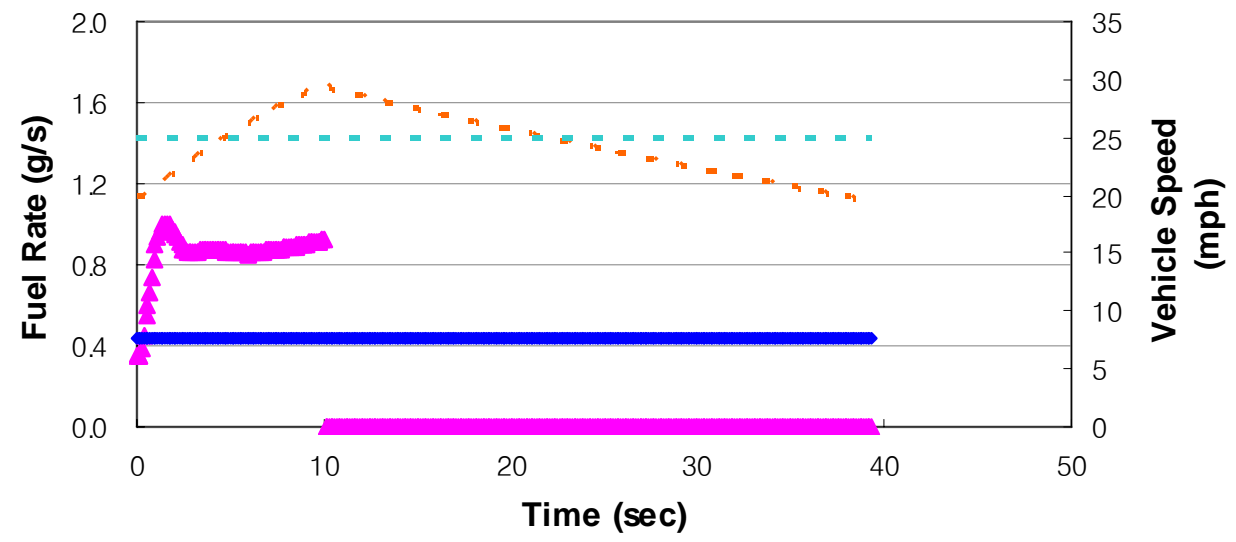

$\Delta \quad$ Fuel Rate: PnG
--- Vehicle Speed: PnG

- Fuel Rate: Steady Speed

- $=$ - Vehicle Speed: Steady Speed

Figure 4.5 Fuel rate comparison between steady speed at $25 \mathrm{mph}$ and $\mathrm{PnG}$ in $20-30 \mathrm{mph}$ with 10 seconds of acceleration in gear 3, 2007 Ford Focus

The cumulative fuel consumption over a drive cycle is also plotted in Figure 4.6. In the steady speed driving condition, the fuel consumption is increased linearly with the constant fuel rate discussed before. In contrast to that, the PnG driving case shows higher cumulative fuel consumption until 10 seconds of acceleration. That cumulative fuel consumption becomes the overall fuel use of the PnG case at the end of the drive cycle. Therefore, the overall fuel consumption of the PnG case is lower than that of the steady speed case. Note that the VMT and overall cycle time for both cases are almost identical. 


\section{Cumulative Fuel Use between Steady Speed at $25 \mathrm{mph}$ and PnG in $20-30 \mathrm{mph}$ w/ 10 Seconds of Acceleration 2007 Ford Focus}

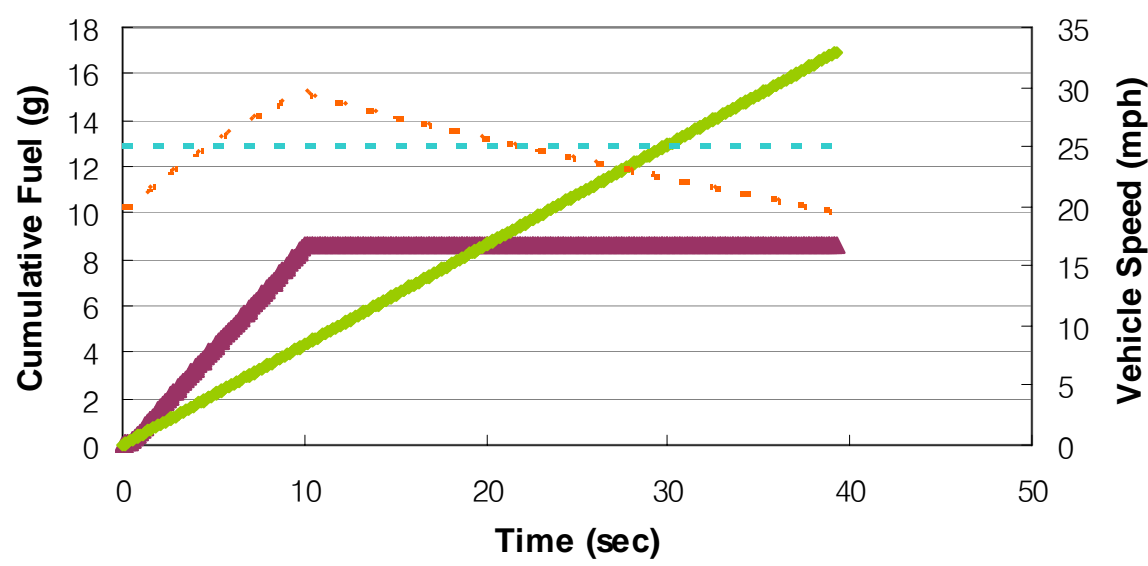

$\Delta \quad$ Cumulative Fuel: PnG $\quad$ Cumulative Fuel: Steady Speed - - - - Vehicle Speed: PnG = - = - Vehicle Speed: Steady Speed

Figure 4.6 Fuel rate comparison between steady speed at $25 \mathrm{mph}$ and $\mathrm{PnG}$ in $20-30 \mathrm{mph}$ with 10 seconds of acceleration in gear 3, 2007 Ford Focus

In Figures 4.5 and 4.6, only one set of PnG and steady speed cases are described but other ones show a similar trend in fuel rate and cumulative fuel consumption. In the same speed range of PnG driving, if the acceleration time is shorter, the fuel rate can be typically increased due to higher torque demand in engine but the cumulative fuel consumption becomes lower up to an optimum point. However, if the acceleration time is too short, in other words, if the torque demand is too high, it is difficult to get the benefit of the PnG driving on fuel consumption. The next section discusses fuel economy benefit of PnG and its limit for 2007 Ford Focus in detail.

\subsubsection{Fuel Economy Results}

Fuel economy is estimated for all selected PnG and steady speed cases using the data in Tables B.5, B.6 and B.7 in Appendix B. Table 4.2 summarizes the fuel economy results for a conventional vehicle, 2007 Ford Focus from PSAT simulation. Note that the 5 seconds of acceleration cases for $30-40 \mathrm{mph}$ and $40-50 \mathrm{mph}$ could not be achieved due to engine torque limit with selected gears. The gears are fixed for each speed range again to avoid the effect of gear shifting on fuel consumption. The steady speed cases are run with an average speed for 
each PnG case; $25 \mathrm{mph}$ for 20 - $30 \mathrm{mph}$ of PnG, $35 \mathrm{mph}$ for 30 - $40 \mathrm{mph}$ of PnG and $45 \mathrm{mph}$ for $40-50 \mathrm{mph}$ of PnG respectively.

Table 4.2 Fuel economy results for 2007 Ford Focus from PSAT simulation

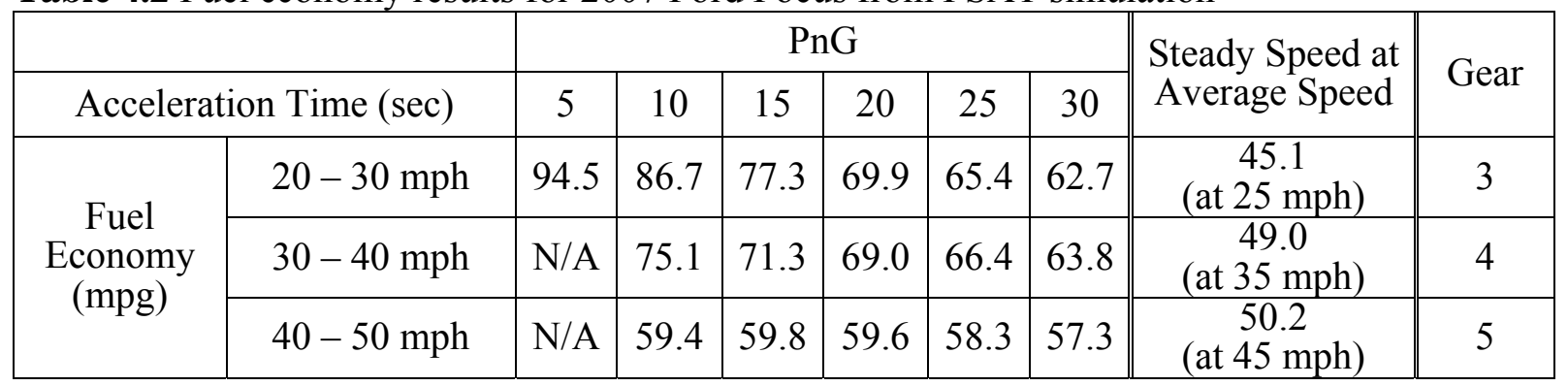

The fuel economy results in Table 4.2 are plotted in Figure 4.7. The dashed lines are for steady speed cases and the solid lines are for PnG cases. The fuel economy results from PSAT simulation are lower the results in Figure 3.13 because engine efficiency is assumed to be constant at a given engine torque in the simple vehicle model. However, the results in Table 4.2 show that the fuel economy of PnG is still higher than that of steady speed and the fuel economy improvement of PnG generally becomes smaller as the acceleration time is longer. For example, the fuel economy of the PnG driving between 20 and $30 \mathrm{mph}$ with 10 seconds of acceleration shows $92 \%$ of improvement compared to that of steady speed driving at $25 \mathrm{mph}$. Note that the 5 seconds of acceleration case in $20-30 \mathrm{mph}$ of $\mathrm{PnG}$ shows $1 \mathrm{mph}$ of lower average speed because the vehicle cannot follow the vehicle speed profile in that short acceleration time. 


\section{Fuel Economy Comparison for Different Acceleration Time 2007 Ford Focus (PSAT)}

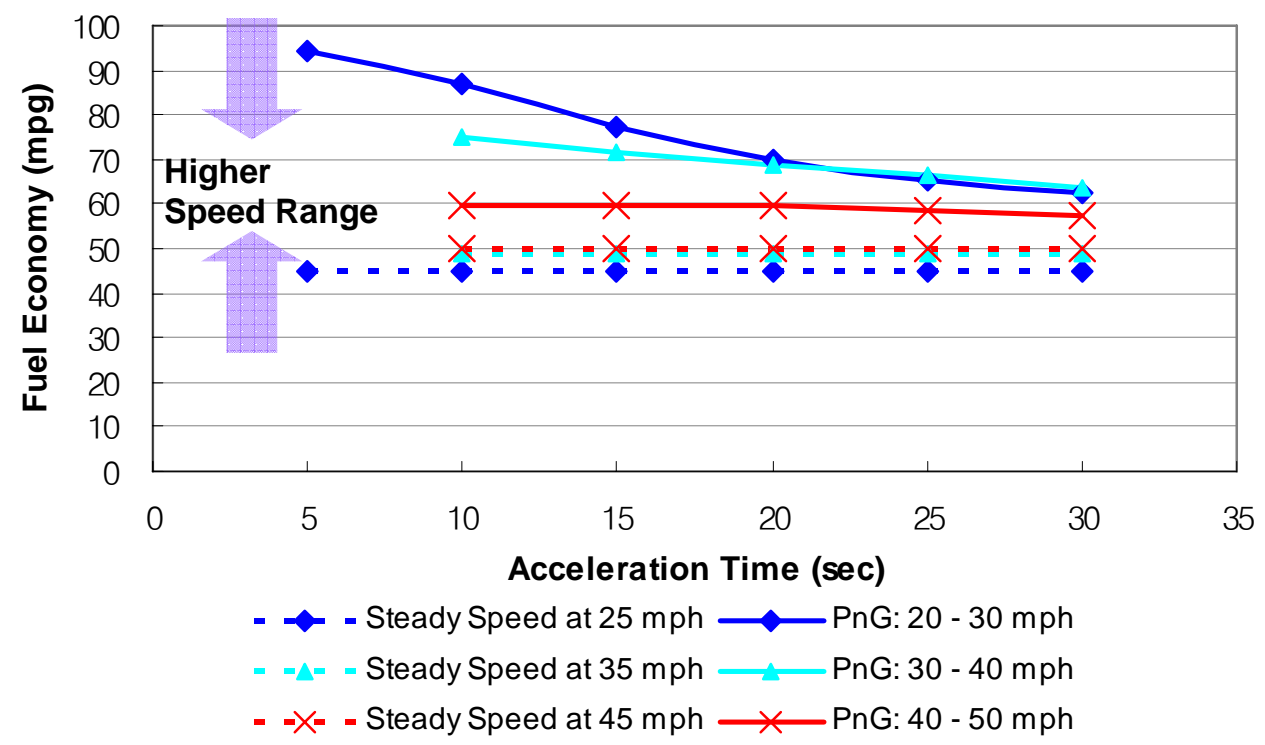

Figure 4.7 Fuel economy comparison for different acceleration time, 2007 Ford Focus

This trend of fuel economy improvement becomes larger as the acceleration is shorter and the speed range is lower. However, the fuel economy at 10 seconds of acceleration in the higher speed range, $40-50 \mathrm{mph}$, shows a little lower fuel economy than that at 15 seconds of acceleration. Also, the fuel economy improvement over all acceleration times is not very large compared to other two speed ranges because the aerodynamic drag force is proportional to the square of vehicle speed and its effect becomes significantly larger at higher speeds as shown in equation (3.1).

\subsubsection{Efficiency of Kinetic Energy Storage}

The efficiency of KE storage introduced in Section 3.4 is calculated using equation (3.33) for the simulation of 2007 Ford Focus in Figure 4.8 below. The overall trend of the efficiency is a little different from that in Figure 3.15 because engine efficiency is not constant in the real vehicle as simulated in PSAT. It shows that the efficiency of KE is over $30 \%$ for all PnG cases. The highest point is $43.4 \%$ for the 25 seconds of acceleration case in $30-40 \mathrm{mph}$ and the lowest point is $31.6 \%$ for the 30 seconds of acceleration case in $20-30 \mathrm{mph}$. In the lower speed range $(20-30 \mathrm{mph})$, the efficiency of KE is higher at shorter acceleration time except the 5 seconds of 
acceleration time case. However, the efficiency of $\mathrm{KE}$ for the other two cases is higher at mild acceleration times; 20 and 25 seconds because engine efficiency for different speed ranges is a little different as explained below.

\section{Efficiency of Kinetic Energy Storage for Different Acceleration \\ Time, 2007 Ford Focus (PSAT)}

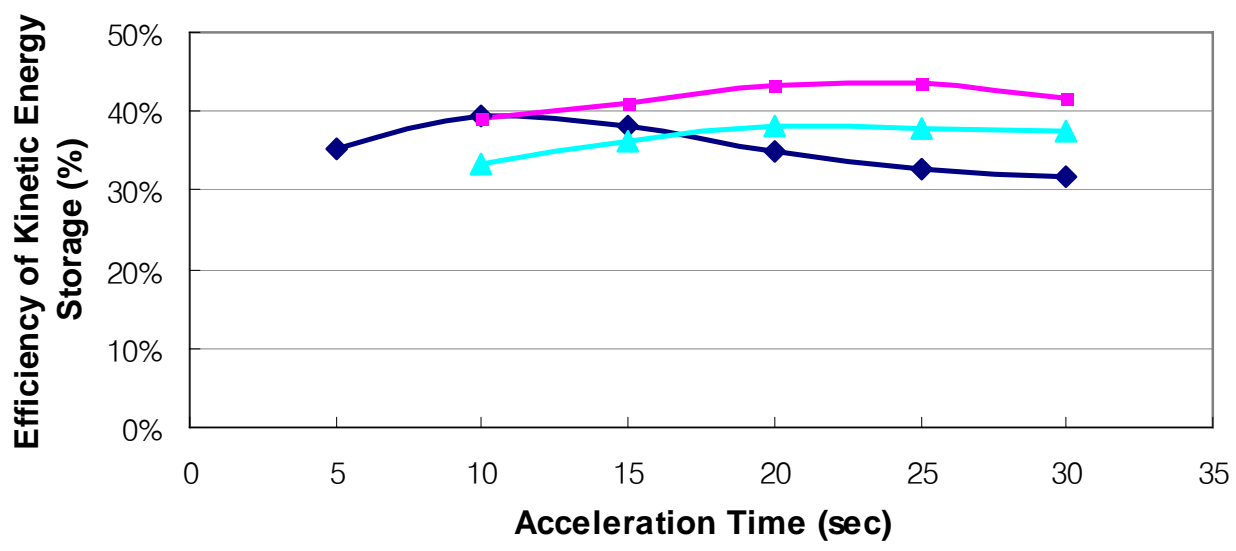

$\longrightarrow$ Eff. of KE of PnG: 20 - $30 \mathrm{mph} \longrightarrow$ Eff. of KE PnG: $30-40 \mathrm{mph}$

- Eff. of KE PnG: 40 - $50 \mathrm{mph}$

Figure 4.8 Efficiency of KE storage for different acceleration time from PSAT, 2007 Ford Focus

Engine Efficiency of PnG during Acceleration

20 - 30 mph, 2007 Ford Focus (PSAT)

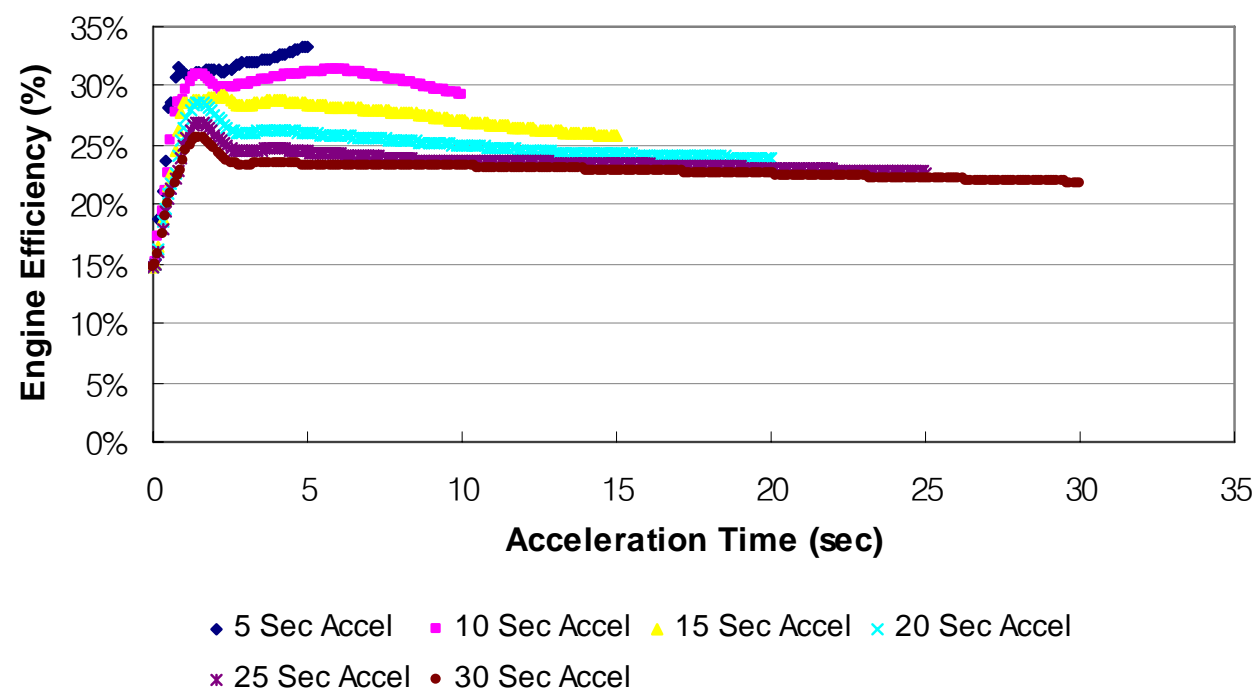

Figure 4.9 Engine efficiency of PnG during acceleration in 20 - $30 \mathrm{mph}$ from PSAT, 2007 Ford Focus 
Figure 4.9 above shows engine efficiency of the PnG cases in $20-30 \mathrm{mph}$ during acceleration as an example. In the beginning of acceleration, engine efficiency is increased from lower range $(\sim 15 \%)$ to higher range in order to produce higher torque. It means that engine load has been increased due to acceleration with increase of engine speed. The other two speed range cases also show very similar trend of engine efficiency over time during acceleration. In addition, the average engine efficiency from the PSAT simulation is higher than the overall engine efficiency (constant for each case) from the simple vehicle model as plotted in Figure 4.10 because engine friction is assumed to be constant in the simple vehicle model whereas it is not constant in real vehicle model of PSAT.

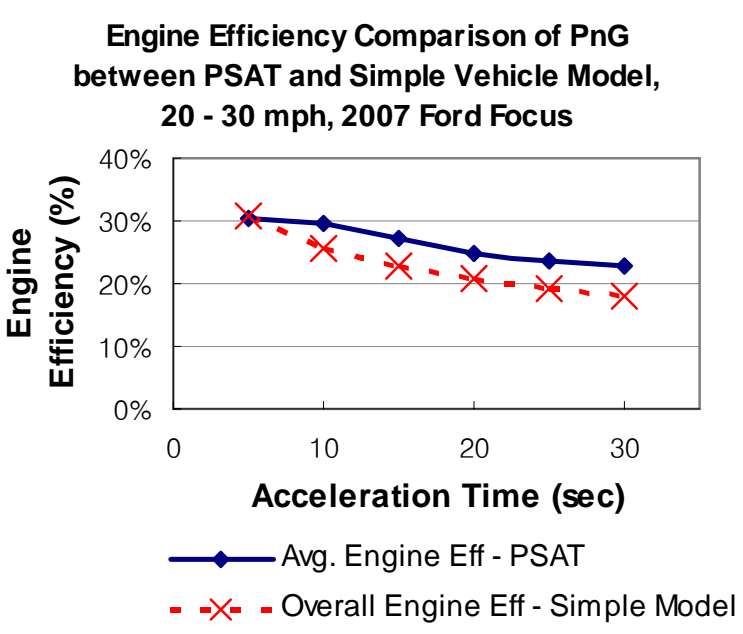

(a) PnG: $20-30 \mathrm{mph}$

\section{Engine Efficiency Comparison of PnG between PSAT and Simple Vehicle Model,}

30 - 40 mph, 2007 Ford Focus

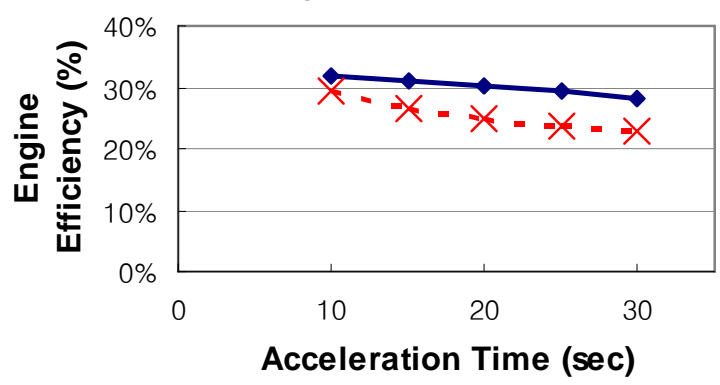

$\sim$ Avg. Engine Eff - PSAT

- -X- - Overall Engine Eff - Simple Model

(b) PnG: $30-40 \mathrm{mph}$

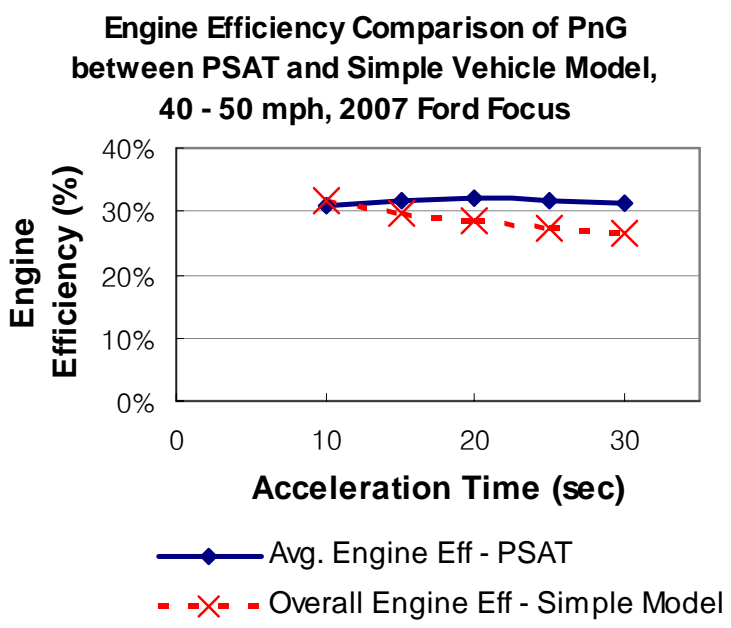

(c) PnG: $40-50 \mathrm{mph}$

Figure 4.10 Engine efficiency of PnG from PSAT and simple vehicle model, 2007 Ford Focus 


\subsection{HEV: 2004 Toyota Prius}

In contrast to conventional vehicles discussed in the previous section, HEVs have some advantages to use the PnG driving strategy. In general, HEVs can turn the engine on and off automatically by their controllers depending on torque demand. In this study, a Toyota Prius (model year: 2004) has been selected because 1.5 L gasoline engine can be turned off to cut off fuel automatically at low speed with low or zero torque demand and a driver does not have to do this manually. It also has a planetary gear set used as a power split device (PSD) that functions as an electric continuously variable transmission (e-CVT) and it is unnecessary to shift up or down while driving, but the engine must be spinning above a certain vehicle speed due to the planetary gear set and motor speed limits. The e-CVT helps avoiding improper gear selection along with the vehicle speed.

The 2004 Toyota Prius HEV is simulated in PSAT to estimate the impact of KE stored in vehicle inertia by the PnG driving strategy. Two more cases; 5 seconds of acceleration times for $30-40$ $\mathrm{mph}$ and $40-50 \mathrm{mph}$ are added and they are listed in Table 4.3 below. The vehicle speed profile of coast down during deceleration is specified to avoid engine compression braking which gives worse fuel economy due to shorter distance and it is also expected that the engine is off during coast down by the HEV control strategy. Again, the drive cycles are generated from the simple vehicle model and with PSAT.

Table 4.3 Selected cases to estimate the fuel economy improvement of PnG and steady speed driving strategies for 2004 Toyota Prius in PSAT

\begin{tabular}{|c||c|c|c|c|c|c|c|}
\hline \multirow{2}{*}{$\begin{array}{l}\text { Steady Speed } \\
(\mathrm{mph})\end{array}$} & \multicolumn{6}{|c|}{ AnG } \\
\cline { 2 - 9 } & Speed Range $(\mathrm{mph})$ & \multicolumn{5}{|c|}{ Acceleration Time (sec) } \\
\hline 25 & $20-30$ & 5 & 10 & 15 & 20 & 25 & 30 \\
\hline 35 & $30-40$ & 5 & 10 & 15 & 20 & 25 & 30 \\
\hline 45 & $40-50$ & 5 & 10 & 15 & 20 & 25 & 30 \\
\hline
\end{tabular}

As mentioned in section 2.5, the SOC balance is very important when analyzing fuel consumption for HEVs and it is difficult to discuss the results with one complete PnG drive cycles used in the conventional vehicle case. Therefore, in the PSAT simulation for 2004 Toyota Prius, repeated PnG drive cycles (over 40 minutes) are used to get accurate fuel consumption 
data. After extraction of data from the results for the valid interval with SOC balance, the overall cycle times for the PnG cases are approximately 30 minutes.

\subsubsection{Vehicle Specifications}

Table 4.4 has the vehicle specifications of 2004 Toyota Prius. Again, the road load coefficients tabulated in Table 4.4 are obtained from annual certification test results and data in EPA [52]. The vehicle specifications in Table 4.4 are used in vehicle testing as well. Note that the units of those coefficients are converted from English units in EPA data to SI units for use in PSAT.

Table 4.4 Vehicle specifications of 2004 Toyota Prius used in PSAT

\begin{tabular}{|l|c||l|c|}
\hline Vehicle Model & Toyota Prius & Generator & Max. 30 kW \\
\hline Model Year & 2004 & Transmission & e-CVT \\
\hline Vehicle Test Mass $(\mathrm{kg})$ & $\begin{array}{c}1474.2 \\
(3250 \mathrm{lb})\end{array}$ & A $(\mathrm{N})$ & 88.59 \\
\hline Engine & $1.5 \mathrm{~L}$ & B (N/(m/s)) & 1.38 \\
\hline Traction Motor & Max. $50 \mathrm{~kW}$ & $\mathrm{C}\left(\mathrm{N} /(\mathrm{m} / \mathrm{s})^{2}\right)$ & 0.36 \\
\hline
\end{tabular}

\subsubsection{Fuel Rate, SOC of Battery and Cumulative Fuel Use}

The HEV has two power sources; a 1.5 L engine and a traction motor/battery, hence the fuel consumption pattern is slightly different because the engine has to charge the battery during coasting if SOC of the battery is at a lower limit. Figure 4.11 shows the fuel rate and the SOC of the battery of a PnG with 10 seconds of acceleration in 20 - 30 mph speed range and steady speed driving at $25 \mathrm{mph}$ at for 2004 Toyota Prius in a short interval from the results. The top two lines in the first figure are vehicle speed of the PnG and the steady speed cases. Again, the fuel rate of the steady speed driving is constant, $0.23 \mathrm{~g} / \mathrm{s}$, in the interval shown and it maintains the same fuel rate over the cycle whereas the fuel rate of the PnG driving shows a similar pattern from that of the 2007 Ford Focus plotted in Figure 4.5. In the coast phase, the vehicle does not consume fuel at all because engine is off as shown in Figure 4.5. Again, the fuel rate of the PnG case is much higher than that of the steady speed case during acceleration but it is zero during coasting. In the second figure below, the SOC of the steady speed case is almost constant 
because the engine always produces power to maintain the vehicle speed as explained above. The SOC of the PnG case looks constant in the interval shown in Figure 4.11, but it increases and decreases in a narrow range as plotted in Figure 4.12.

Fuel Rate and SOC Comparison between Steady Speed at 25 mph and PnG in 20 - 30 mph w/ 10 Seconds of Acceleration 2004 Toyota Prius

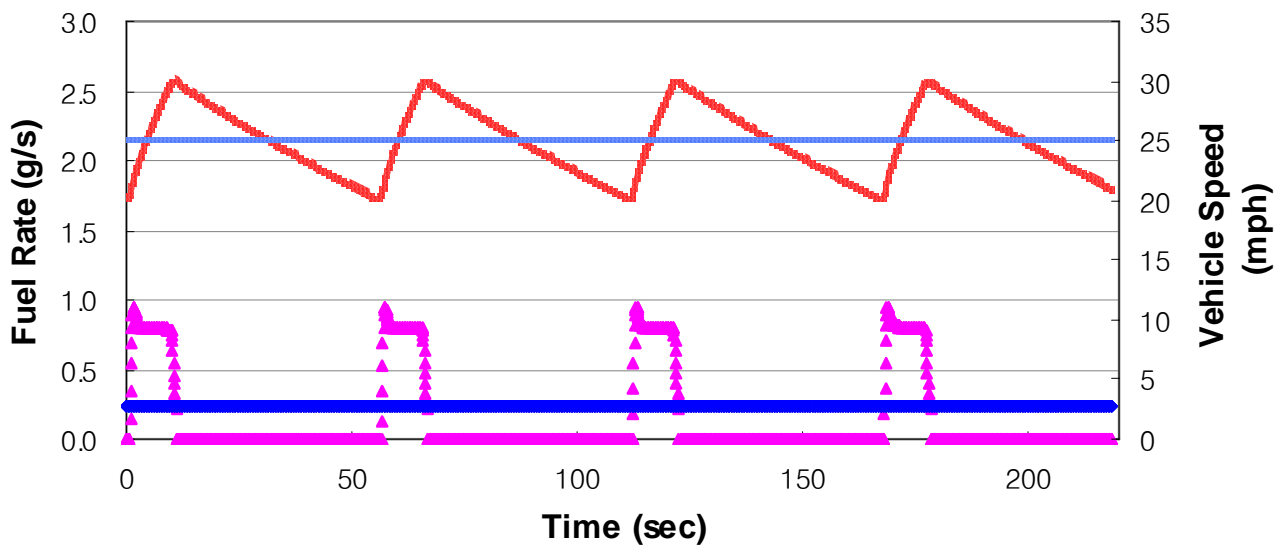

\ Fuel Rate: PnG Vehicle Speed: PnG

- Fuel Rate: Steady Speed Vehicle Speed: Steady Speed

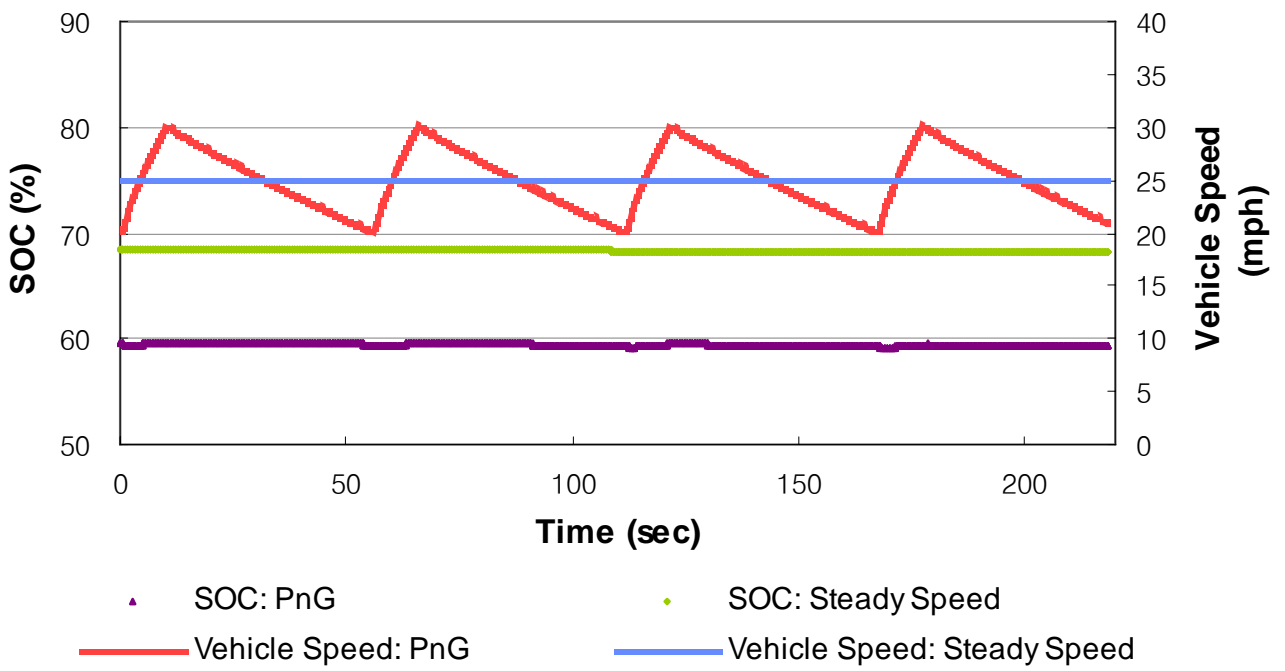

Figure 4.11 Fuel rate comparison between steady speed at $25 \mathrm{mph}$ and PnG in 20 - $30 \mathrm{mph}$ with 10 seconds of acceleration, 2004 Toyota Prius

In Figure 4.12, the SOC of the battery and the battery output power of the same PnG case are plotted as well with the vehicle speed and the fuel rate. Note that the negative values in the 
second plot of Figure 4.12 mean that the battery is being charged. In the beginning of acceleration of each PnG cycle, the battery output power increases dramatically in a very short period of time which means the motor produces power first to propel the vehicle until the engine turns on because the engine is off during coasting. Once the engine turns on and consumes fuel, the SOC is increased a little bit during acceleration. It represents that the engine produces extra power not only to propel the vehicle but also to charge the battery. The SOC is slightly decreased during coasting because the vehicle consumes electric energy for accessories and the amount of battery output power is approximately $0.2 \mathrm{~kW}$.

Fuel Rate, Vehicle Speed, SOC and Battery Ouput Power of PnG in $20-30 \mathrm{mph} \mathrm{w/} 10$ Seconds of Acceleration 2004 Toyota Prius

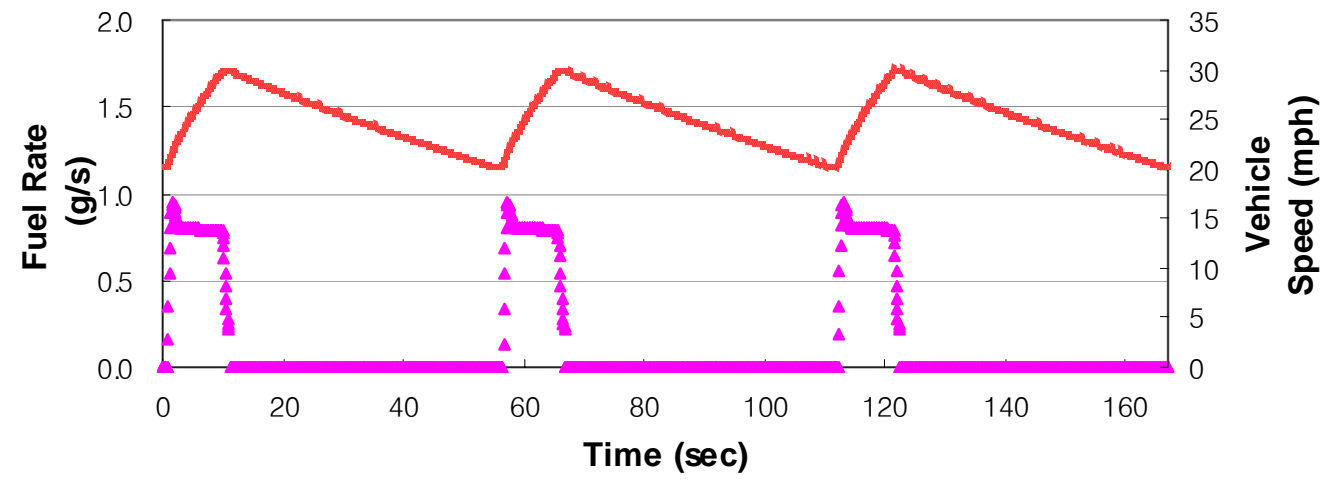

$\Delta \quad$ Fuel Rate $=$ Vehicle Speed

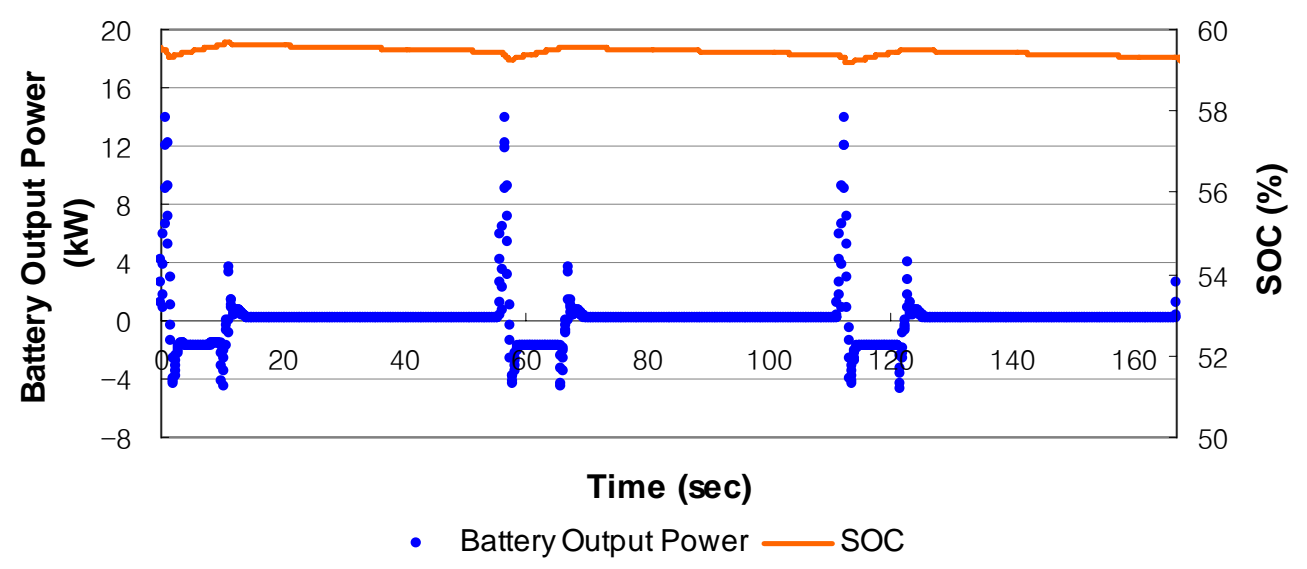

Figure 4.12 SOC and battery output power of PnG in 20 - 30 mph with 10 seconds of acceleration, 2004 Toyota Prius 
Most of the PnG cases for 2004 Toyota Prius show a very similar trend of fuel rate as shown in Figures 4.11 and 4.12. However, a different fuel consumption trend appears in a few PnG cases. Figure 4.13 shows the PnG case for the 5 seconds of acceleration time in $20-30 \mathrm{mph}$. In this case, a high acceleration rate and power demand requires more motor and battery power assist for the engine, and it causes some battery energy discharge. Also, the HEV control strategy to keep the SOC balanced in a relatively narrow range $(\sim 1 \%)$ then causes the engine to run to charge the battery during the coast phase.

\section{Fuel Rate, Vehicle Speed, SOC and Battery Ouput Power of PnG} in 20 - 30 mph w/ 5 Seconds of Acceleration 2004 Toyota Prius

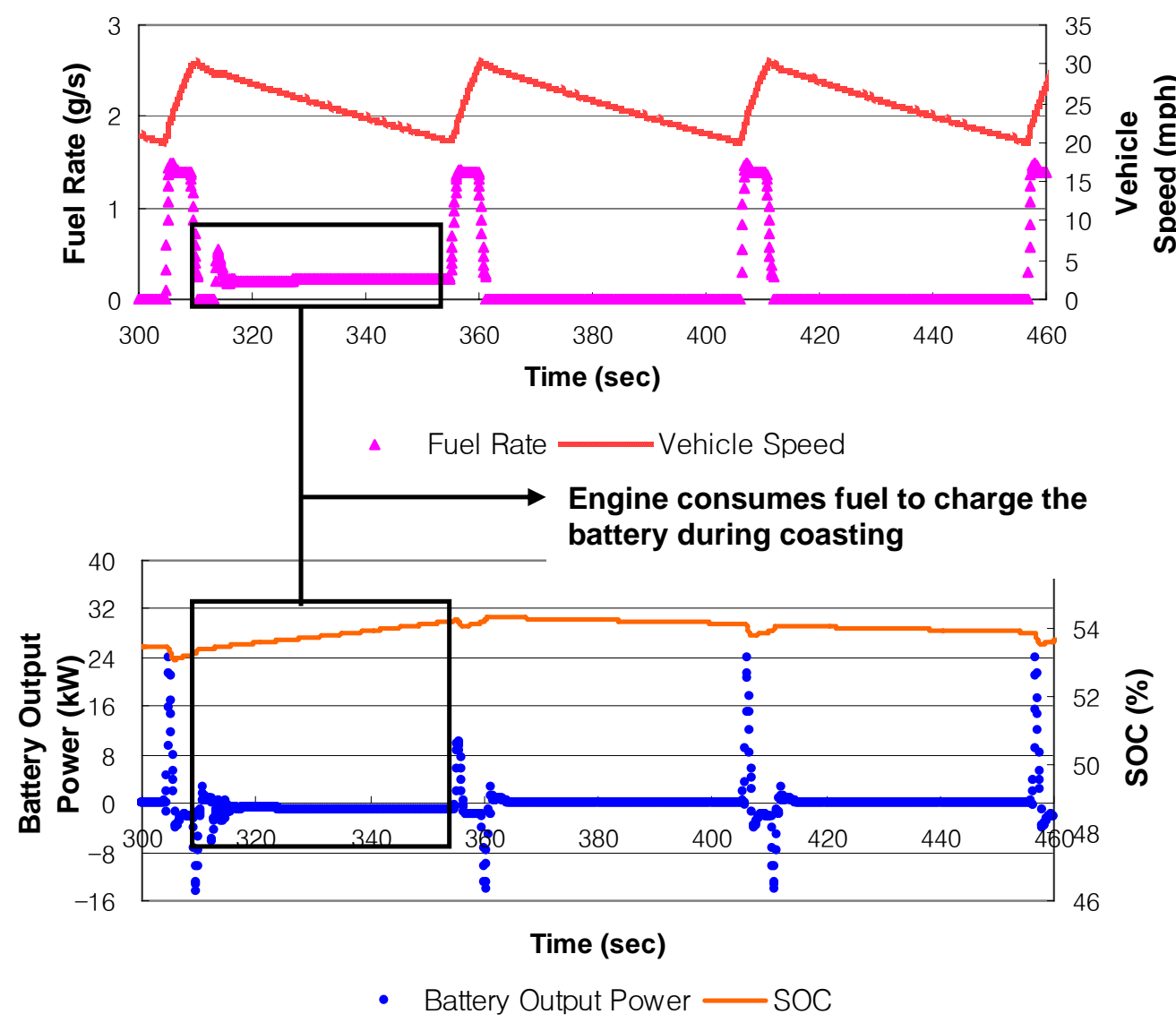

Figure 4.13 Fuel rate, SOC and battery output power of PnG in $20-30 \mathrm{mph}$ with 5 seconds of acceleration, 2004 Toyota Prius

For this particular case, the motor/battery round trip losses while discharging the battery during acceleration and just charging the battery with low engine load during coasting can be higher 
than the loss when transmitting engine load directly to the ground. As shown in the second plot of Figure 4.13, the battery output power is negative and the SOC is increased. The SOC is decreased a little steeper than the slower acceleration cases because more power is required to accelerate the vehicle faster and the motor needs higher power before the engine turns on. The peak power of the battery in the beginning of acceleration is about $24 \mathrm{~kW}$ over the PnG drive cycle. This kind of trend is shown in the PnG cases for the 5 seconds of acceleration time in $30-$ $40 \mathrm{mph}$ and $40-50 \mathrm{mph}$. The fuel consumption during coasting in those cases results in worse fuel economy compared to the 10 seconds of acceleration cases. This case is described in detail in section 4.3.3.

The other example of different fuel consumption trend is plotted in Figure 4.14 below. The fuel consumption during coasting is very similar to the case discussed above whereas it happens more frequently. In addition, the battery power is used to generate tractive power by the motor for a few seconds during acceleration because the tractive power at wheels for 30 seconds of acceleration time is lower then shorter acceleration times. As mentioned previously, if a driver's demand is low at low speed, the HEV is in electric only mode (EV mode) to prevent the engine from operating in low efficiency region and save more fuel. The EV mode for a few seconds is shown in Figure 4.14. However, the results show that the fuel economy is dramatically lower from its trend in these particular PnG drive cycles as shown in Figure 4.16 in Section 4.3.3. If the acceleration time is longer (slower acceleration) than 30 seconds, the HEV can be in the EV mode more frequently. 
Fuel Rate, Vehicle Speed, SOC and Battery Ouput Power of PnG

in $20-30$ mph w/ 30 Seconds of Acceleration

2004 Toyota Prius

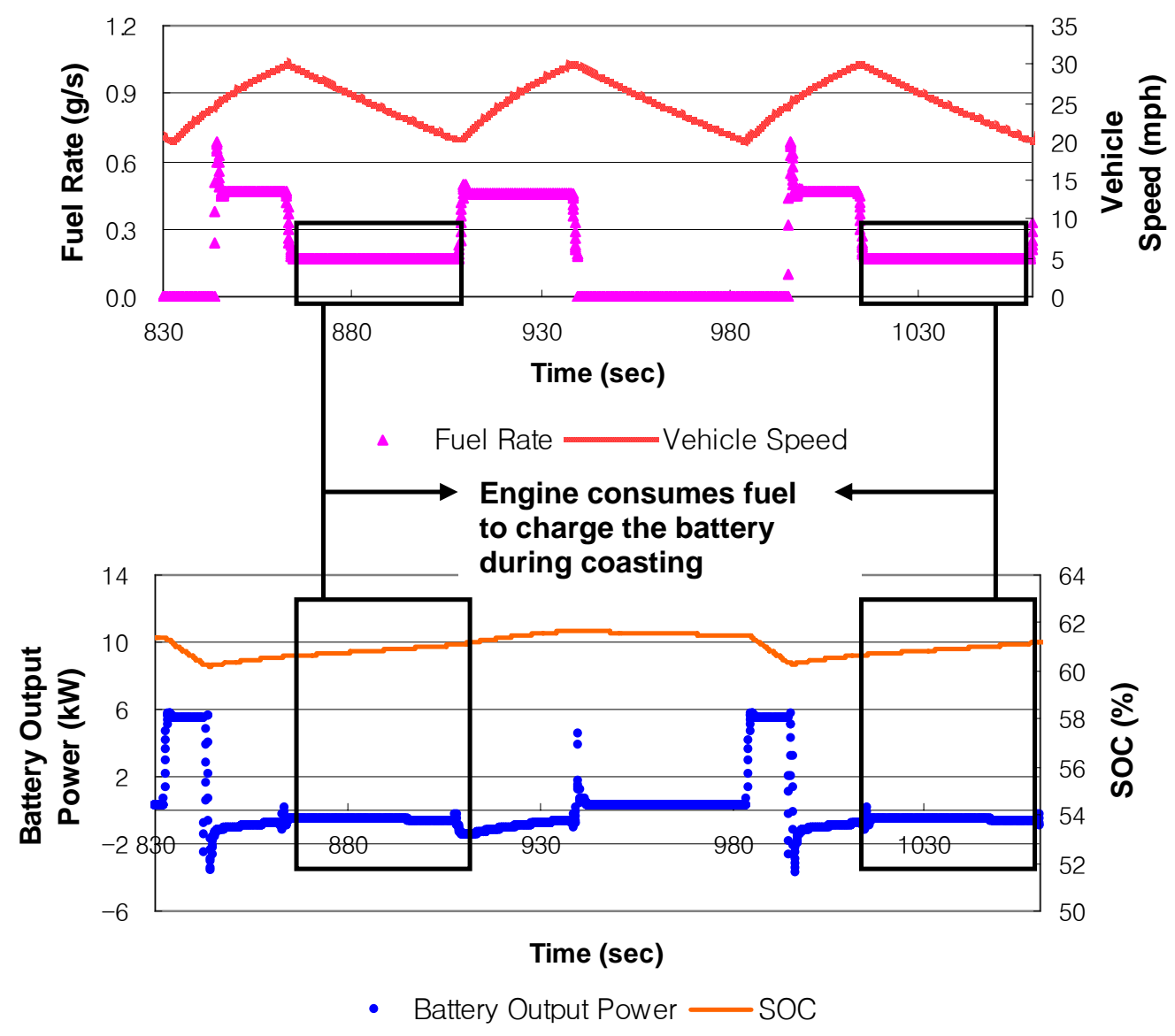

Figure 4.14 Fuel rate, SOC and battery output power of PnG in $20-30 \mathrm{mph}$ with 30 seconds of acceleration, 2004 Toyota Prius

The cumulative fuel consumption of the HEV over a repeated PnG drive cycle is shown in Figure 4.15. It is an example of 18 different PnG cases. The cumulative fuel use for both PnG and steady speed cases show linear increase but if only one cycle of the repeated cycles is observed, then it looks like the cumulative fuel use of a conventional vehicle in Figure 4.6. As a matter of fact, the PnG case in Figure 4.15 shows step increases of fuel use. In the steady speed case, the cumulative fuel use increases linearly with almost a constant fuel rate discussed before (the fuel rate changes on the order of $0.005 \mathrm{~g} / \mathrm{s}$ due to charging the battery when the SOC is low). In contrast to that, the PnG case shows lower cumulative fuel consumption at the end of the 
repeated drive cycle. Therefore, the overall fuel consumption of the PnG case is much lower than that of the steady speed case. The detailed results are summarized in Tables B.8, B.9 and B.10 in Appendix B.
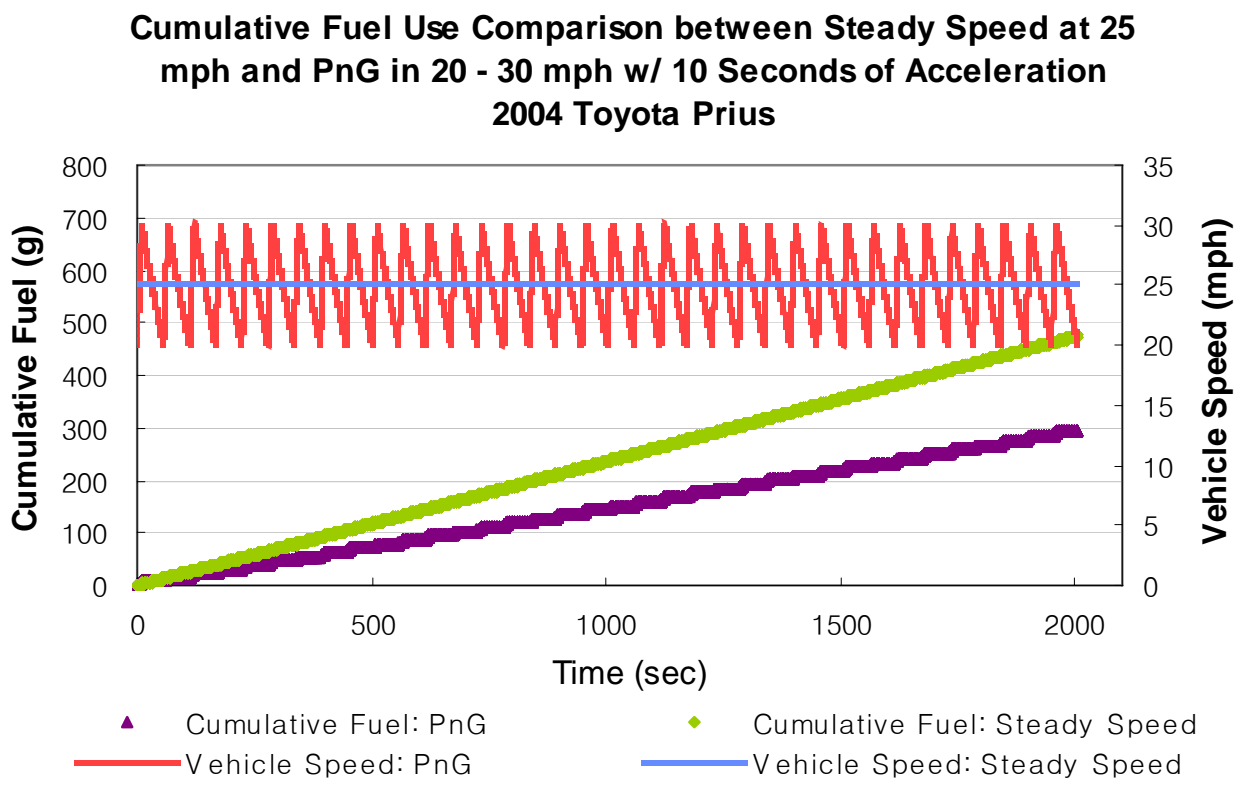

Figure 4.15 Cumulative fuel use comparison between steady speed at $25 \mathrm{mph}$ and PnG in 20 $30 \mathrm{mph}$ with 10 seconds of acceleration in gear 3, 2004 Toyota Prius

In Figure 4.15, only one set of PnG and steady speed cases are discussed but other cases have a similar trend with respect to cumulative fuel use. In the same speed range of PnG driving, if the acceleration time is shorter, the fuel rate and battery output power (in the beginning of acceleration) can be generally increased due to higher torque demand in engine and motor respectively but the cumulative fuel consumption becomes lower to an optimum point ( $\sim 10$ seconds of acceleration for this HEV). However, if the acceleration time is too short ( 5 seconds of acceleration), in other words, if the torque demand is too high, it is difficult to get the benefit of the PnG driving on fuel consumption because the HEV needs higher battery/motor power assist during acceleration and the battery should be charged with low engine load as seen in Figure 4.13. In this case, the motor/battery round trip losses occur and it results in more fuel consumption. The following section discusses fuel economy benefit of PnG and its limit for 2004 Toyota Prius. 


\subsubsection{Fuel Economy Results for Repeated Cycles}

The fuel economy of PnG and steady speed cases for 2004 Toyota Prius is obtained by extracting the data with the method introduced in section 4.1.3. The overall cycle time for the valid data of all the cases is over 30 minutes and the difference of initial and final SOCs is within $\pm 1 \%$ as tabulated in Tables B.8, B.9 and B.10 in Appendix B. Again, 2004 Toyota Prius uses an e-CVT which is not a conventional multi speed transmission and it operates as a CVT so gear selection is not mentioned in this section.

The summary of fuel economy results for the HEV is shown in Table 4.5. The fuel economy results in Table 4.5 are plotted in Figure 4.16 below. The fuel economy of steady speed cases is decreased as the vehicle speed is increased. This trend is similar to the fuel economy of the PnG cases for different speed ranges. The highest fuel economy improvement, $62 \%$, happens between the PnG case of 10 seconds of acceleration in $20-30 \mathrm{mph}$ and the steady speed case at $25 \mathrm{mph}$. Also, the PnG cases with 10 seconds of acceleration in $30-40 \mathrm{mph}$ and $40-50 \mathrm{mph}$ show $44 \%$ and $33 \%$ higher fuel economy than steady speed cases at $35 \mathrm{mph}$ and $45 \mathrm{mph}$ respectively. The lowest fuel economy improvement is only $4 \%$ for the PnG case with 5 seconds of acceleration in $40-50 \mathrm{mph}$ compared to the steady speed case at $45 \mathrm{mph}$.

Table 4.5 Fuel economy results for 2004 Toyota Prius from PSAT simulation

\begin{tabular}{|c|c|c|c|c|c|c|c|c|}
\hline \multirow{2}{*}{\multicolumn{2}{|c|}{ Acceleration Time (sec) }} & \multicolumn{6}{|c|}{$\mathrm{PnG}$} & \multirow{2}{*}{$\begin{array}{l}\text { Steady Speed } \\
\text { at Average } \\
\text { Speed } \\
82.1\end{array}$} \\
\hline & & 5 & 10 & 15 & 20 & 25 & 30 & \\
\hline \multirow{3}{*}{$\begin{array}{l}\text { Fuel } \\
\text { Economy } \\
(\mathrm{mpg})\end{array}$} & $20-30 \mathrm{mph}$ & 113.7 & 133.3 & 132.1 & 124.4 & 120.2 & 99.1 & $\begin{array}{c}82.1 \\
\text { (at } 25 \mathrm{mph} \text { ) }\end{array}$ \\
\hline & $30-40 \mathrm{mph}$ & 92.1 & 109.4 & 107.3 & 103.0 & 100.0 & 97.9 & $\begin{array}{c}76.1 \\
\text { (at } 35 \mathrm{mph} \text { ) }\end{array}$ \\
\hline & $40-50 \mathrm{mph}$ & 68.2 & 87.2 & 86.0 & 83.0 & 80.7 & 78.2 & $\begin{array}{c}65.4 \\
\text { (at } 45 \mathrm{mph} \text { ) }\end{array}$ \\
\hline
\end{tabular}

The interesting case for the HEV is the PnG driving with 30 seconds of acceleration in $20-30$ mph. The fuel economy drops significantly compared to the other PnG cases and it shows relatively low fuel economy improvement, $21 \%$. As explained in previous section, if a driver's demand is low (low acceleration) at low speed $(<40 \mathrm{mph})$, the HEV uses the motor and battery instead of just the engine to propel the vehicle and save fuel. However, the SOC becomes low due to frequent use of battery power in EV mode during acceleration and the HEV control strategy keeps the SOC balanced, so the engine has to run to charge the battery during the coast 
phase as seen in Figure 4.14. It finally results in more fuel consumption over the PnG drive cycle. As shown in Figure 4.17 - (a), the HEV consumes more fuel to charge the battery during coasting than other PnG cases. In general, the EV mode can be more efficient in most of vehicle use rather than PnG driving.

Fuel Economy Comparison between PnG and Steady Speed from PSAT, 2004 Toyota Prius
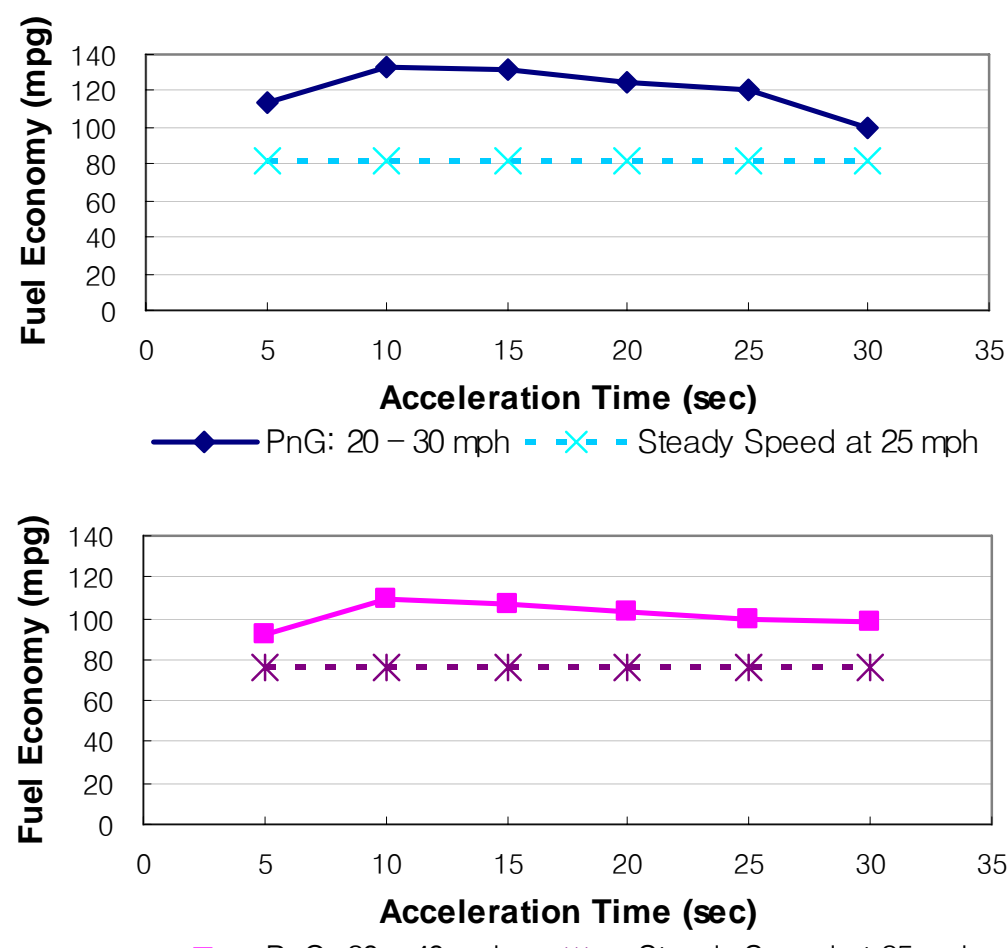

$\longrightarrow$ PnG: 30 - 40 mph - -*- - Steady Speed at 35 mph

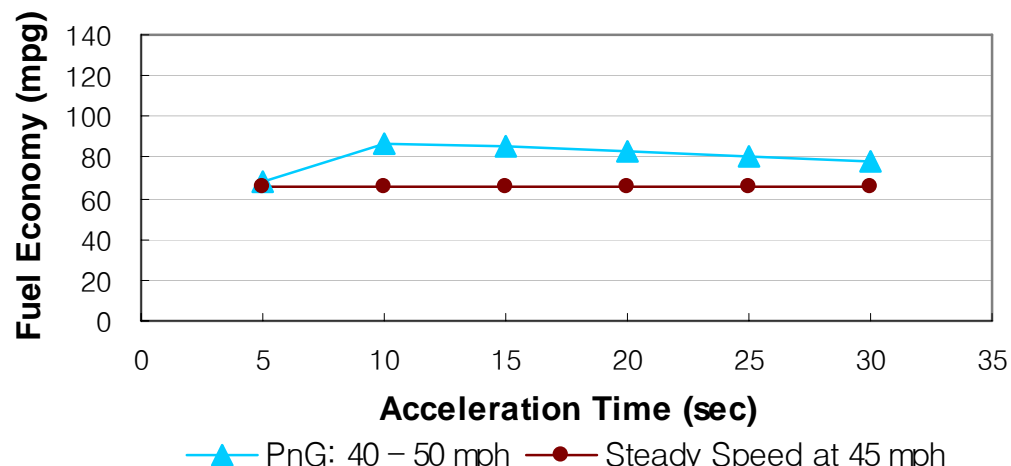

Figure 4.16 Fuel economy comparison for different acceleration time, 2004 Toyota Prius 
Fuel Use Comparison during Acceleration and Coasting from PSAT, 2004 Toyota Prius

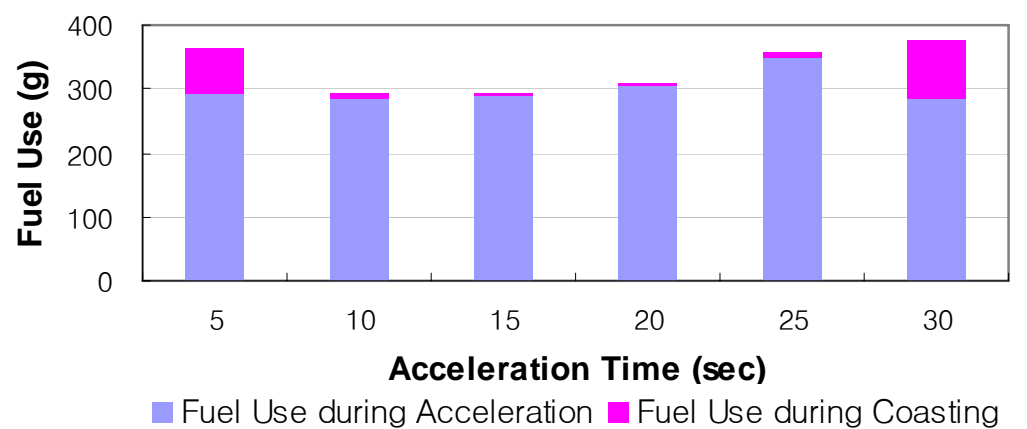

(a) PnG: 20 - $30 \mathrm{mph}$

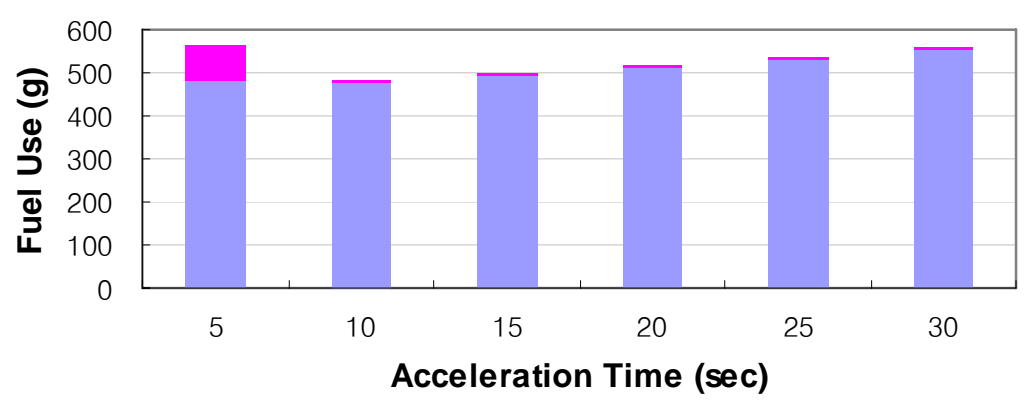

- Fuel Use during Acceleration Fuel Use during Coasting

(b) PnG: 30 - $40 \mathrm{mph}$

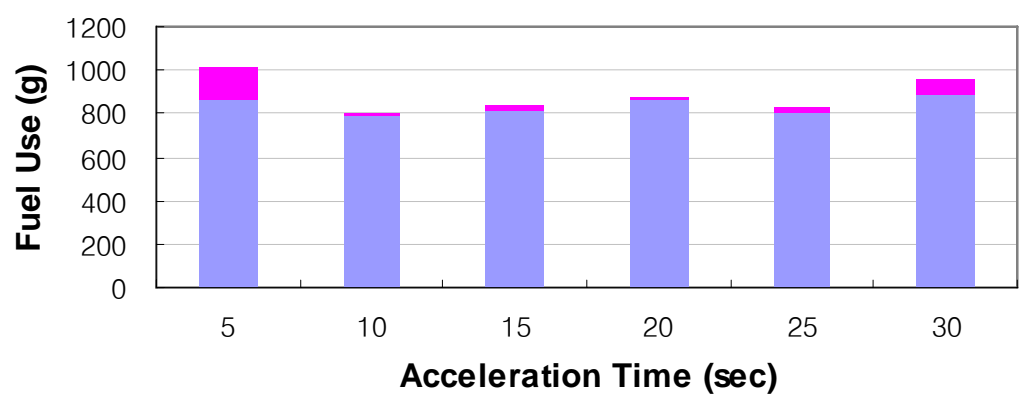

Fuel Use during Acceleration Fuel Use during Coasting

(c) PnG: 40 - $50 \mathrm{mph}$

Figure 4.17 Fuel use comparison during acceleration and coasting, 2004 Toyota Prius

One more different fuel economy trend in Figure 4.16 for the HEV compared to the conventional vehicle is the 5 seconds of acceleration cases. As shown in Figure 4.7, the fuel economy of the 5 seconds of acceleration case in $20-30 \mathrm{mph}$ for the conventional vehicle is even better than that of the 10 seconds of acceleration in the same speed range. However, the fuel economy of the 5 
seconds of acceleration cases in Figure 4.16 is much worse than that of the 10 seconds of acceleration cases. As discussed previously, if the acceleration time is too short, the motor uses more battery electric power in the beginning of acceleration until the engine turns on or assists the engine to generate more power to propel the vehicle. Thus, the SOC of the battery drops and the engine has to consume fuel to charge the battery during coasting. This causes more fuel consumption over the repeated PnG drive cycles. The trend can be seen in Figure 4.17 above. In summary, after 10 seconds of acceleration, the fuel economy of the PnG decreases as the acceleration time becomes longer because the HEV is occasionally in EV mode during acceleration and the engine has to charge the battery again if the SOC of the battery becomes low. Again, the round trip losses between the engine/motor and the battery become larger in long acceleration time (mild acceleration). Also, the fuel economy improvement of the PnG driving decreases as the speed range is higher. In steady speed driving, the HEV mostly uses the engine power to propel the vehicle without engine load leveling in PSAT simulation and the engine efficiency is lower than PnG driving, so it results in lower fuel economy.

\subsubsection{Efficiency of Kinetic Energy Storage}

The efficiency of KE storage in vehicle inertia is also calculated for the HEV from the results by PSAT and shown in Figure 4.18. Again, the efficiency of KE is higher than the engine efficiency of the vehicle. The engine efficiency is summarized in Tables B.8, B.9 and B.10 in Appendix B. The trend of the efficiency of KE storage is a little different from that in Figure 4.8 for the conventional vehicle. Especially, the PnG case with 30 seconds of acceleration in $20-30$ mph shows an increase of the efficiency of KE because the HEV uses the motor only for a while to propel the vehicle during acceleration as explained previously. From this case, it is found that the efficiency of KE storage is higher if the HEV is in EV mode during acceleration because the difference of fuel consumption between the PnG and the steady speed cases during acceleration becomes smaller in EV mode as expressed in equation (3.33). In other words, if the HEV should consume more fuel during coasting to charge the battery after discharging the electric power in EV mode, the efficiency of KE storage can be increased. Therefore, this term should be carefully analyzed and understood for the HEVs. 


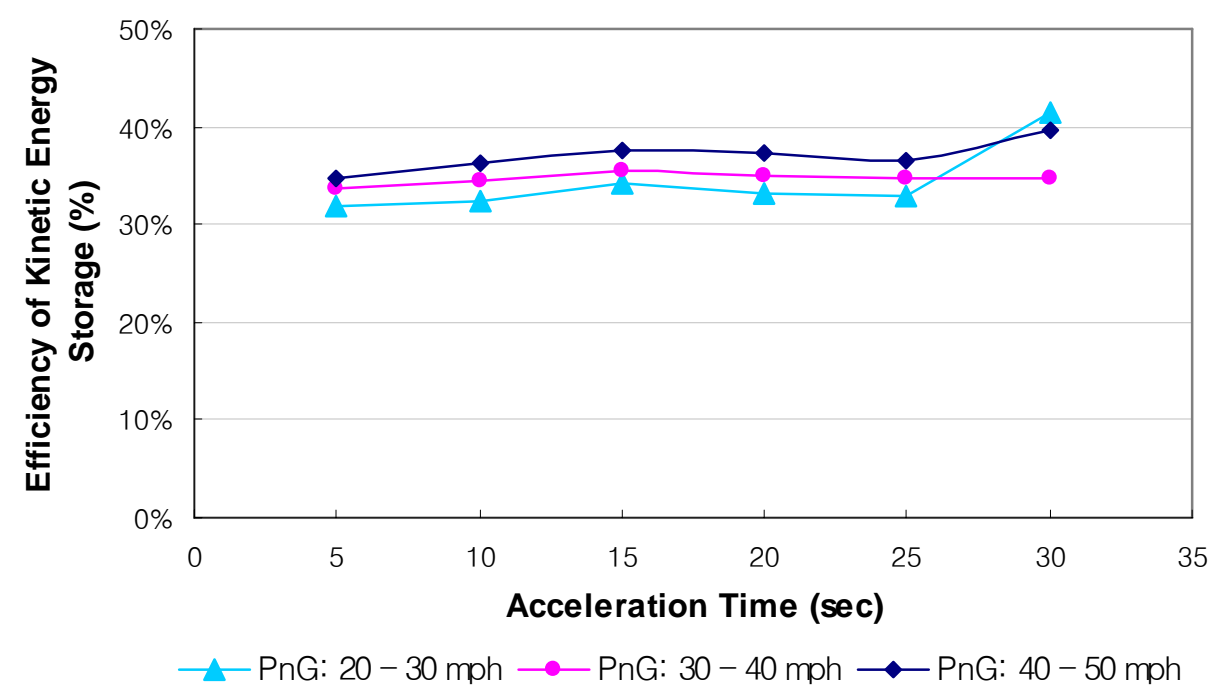

Figure 4.18 Efficiency of KE storage, 2004 Toyota Prius

\subsection{Summary}

In Chapter 4, the PnG driving strategy is simulated for the conventional vehicle, 2007 Ford Focus, and the HEV, 2004 Toyota Prius in PSAT to find its impact on fuel economy and the results are compared to the steady speed cases. The results for the conventional vehicle and the HEV show some difference for each case, however there is large improvement of fuel economy with the PnG driving strategy compared to steady speed results. In general, the fuel economy of the PnG driving decreases as the acceleration time is longer and increases as the speed range is lower for both the conventional vehicle and the HEV. However, the HEV shows that the fuel economy can be worse if the acceleration time is too short ( 5 seconds) because the engine has to consume fuel during coasting to charge the battery due to electric power consumption during acceleration. In addition, the fuel economy improvement by the PnG driving drops significantly for the 30 seconds of acceleration case in $20-30 \mathrm{mph}$ because the HEV uses motor only for a few seconds to propel the vehicle during acceleration and the engine again consumes fuel to charge the battery during coasting. The fuel economy improvement by PnG driving strategies is smaller in the HEV than in the conventional vehicle because the HEV has energy storage, the 
battery, and it does engine load leveling depending on driving conditions. From the vehicle simulation using PSAT, the PnG driving strategy clearly shows some potential to improve fuel over the steady speed driving. In order to verify the simulation results discussed so far, vehicle testing for the HEV is designed and performed at ANL. The experimental setup and the results from the vehicle testing are described in detail in Chapter 5 and Chapter 6 respectively. 


\section{Chapter 5 Experimental Setup and Vehicle Testing for an HEV}

The simulation results by the simple vehicle model and the PSAT simulation clearly show potential of fuel economy improvement by the PnG driving strategy compared to the steady speed driving cases. Especially, fuel economy can be improved much more at low speed range and higher acceleration because aerodynamic drag force is smaller at low speed and engine is operating in more efficient region in short period of time respectively except 5 seconds of acceleration time case for an HEV. The HEV, 2004 Toyota Prius was tested at ANL to verify the improvement of fuel economy by the PnG driving strategy. This chapter describes the experimental setup in a test facility of ANL and discusses how the test cases are designed in the following section.

\subsection{Experimental Setup}

The vehicle testing is performed with a 2004 Toyota Prius on a four wheel drive (4WD) dynamometer in the Advanced Powertrain Research Facility (APRF) at ANL [53]. The test vehicles are warmed up before testing the selected $\mathrm{PnG}$ drive cycles on the dynamometer to avoid cold start impact. The cold engine start generally affects both fuel consumption and emissions of a vehicle [54]. Also, the ambient temperature of the test cell can affect the fuel consumption [55] and emissions [56] so the temperature in the test cell is maintained at $22.5{ }^{\circ} \mathrm{C}$ $\left(72.5^{\circ} \mathrm{F}\right)$ average. The relative humidity is also controlled between $43.1 \%$ and $47.9 \%$ as shown 
in Table 5.1. Note that EPA Tier 2 EEE certified gasoline is used for testing and its density value, $0.741 \mathrm{~g} / \mathrm{ml}$, and specific energy, $18386.0 \mathrm{BTU} / \mathrm{lbm}$, are used for simulation as well. Figure 5.1 shows the test vehicle on a dynamometer in ANL.

Table 5.1 Basic summary of vehicle testing condition in a test cell

\begin{tabular}{|l|c||l|c|}
\hline Average Temperature $\left({ }^{\circ} \mathrm{C}\right)$ & 22.5 & Fuel Type & $\begin{array}{c}\text { EPA Tier 2 EEE } \\
\text { certified gasoline }\end{array}$ \\
\hline Relative Humidity (\%) & $43.1-47.9$ & $\begin{array}{l}0.741 \mathrm{~g} / \mathrm{ml} \\
\text { Density, } \\
\text { Energy Content }\end{array}$ & $\begin{array}{c}18386.0 \mathrm{BTU} / \mathrm{lbm} \\
(42.77 \mathrm{MJ} / \mathrm{kg})\end{array}$ \\
\hline
\end{tabular}

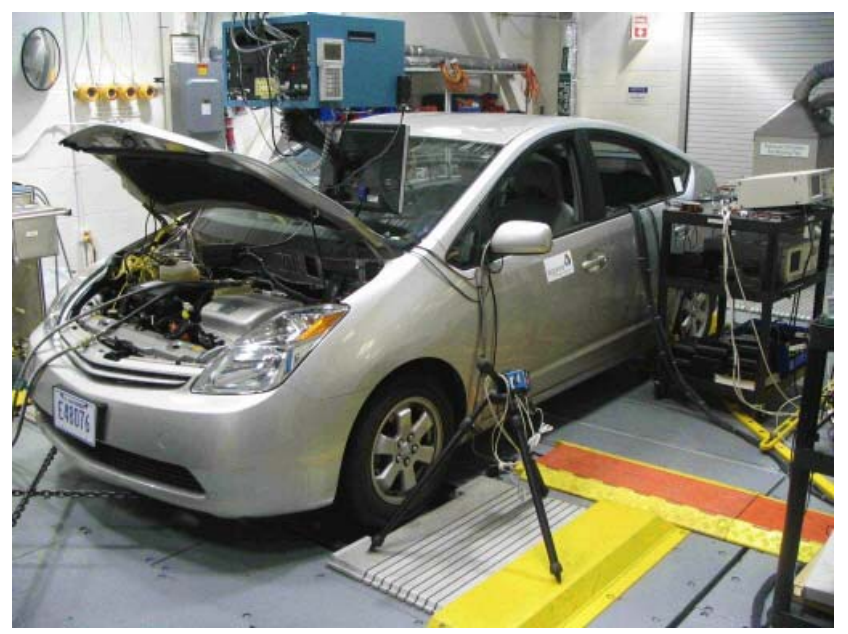

Figure 5.1 Test vehicle, 2004 Toyota Prius, on a dynamometer in ANL

The schematic of the vehicle testing facility in ANL is illustrated in Figure 5.2. The test vehicle is set up on the dynamometer and a test driver tries to control the accelerator pedal to follow a speed trace or drive cycle displayed on the screen. The experimental data is transmitted to a data acquisition system (DAQ) through a control-area network (CAN) in a control room and recorded in a computer. Note that the CAN is a vehicle bus standard embedded on a vehicle so controllers can communicate with each other without a host computer. Also it can transmit vehicle data such as engine power, engine torque, engine speed, fuel rate and vehicle speed to a data logging system.

There are two levels of vehicle testing available in ANL; level 1 and level 2. The level 1 is collecting basic data from over 100 parameters such as time, vehicle speed, dynamometer force, ambient temperature, engine power / torque, battery power and emissions. In addition to the 
level 1 test, more data can be obtained in the level 2 test through additionally instrumented equipment, for example, a torque sensor installed in a transmission. In this study, only level 1 testing is conducted to collect data from the vehicle testing because extra data and instrumentation is not necessary for the purpose of the study.

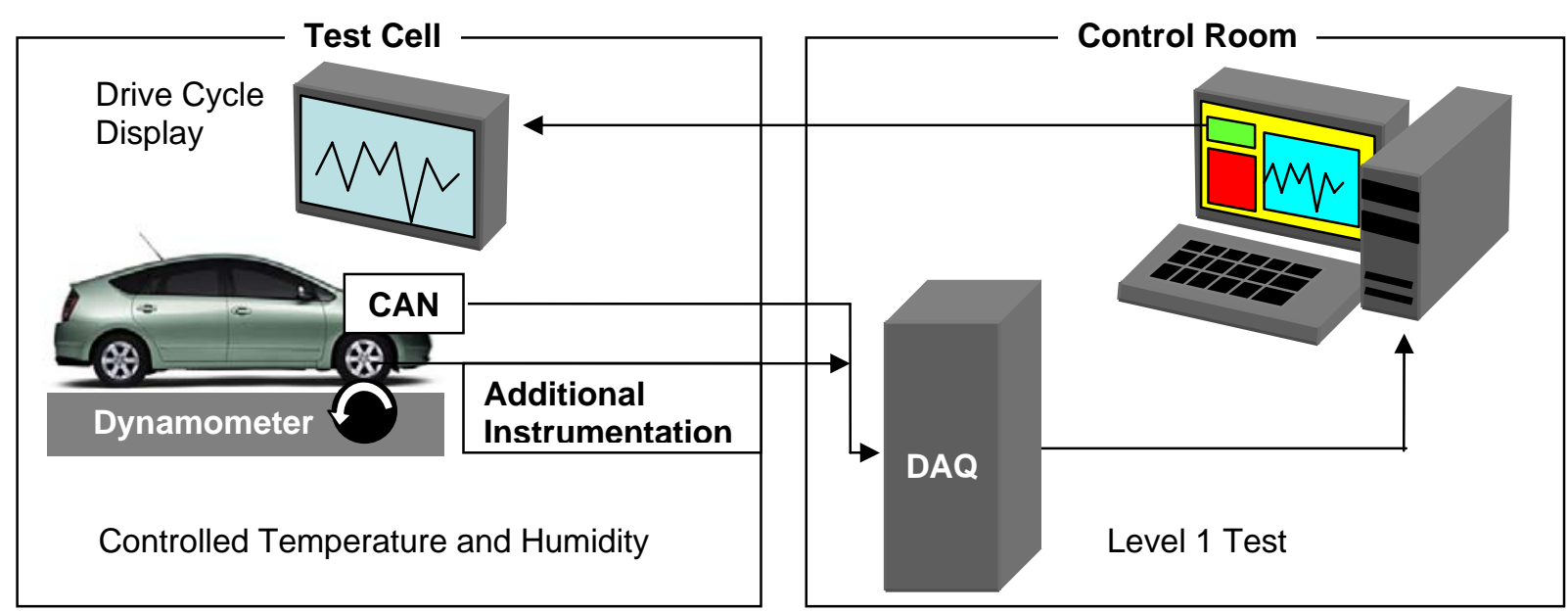

Figure 5.2 Schematic of vehicle testing facility in ANL

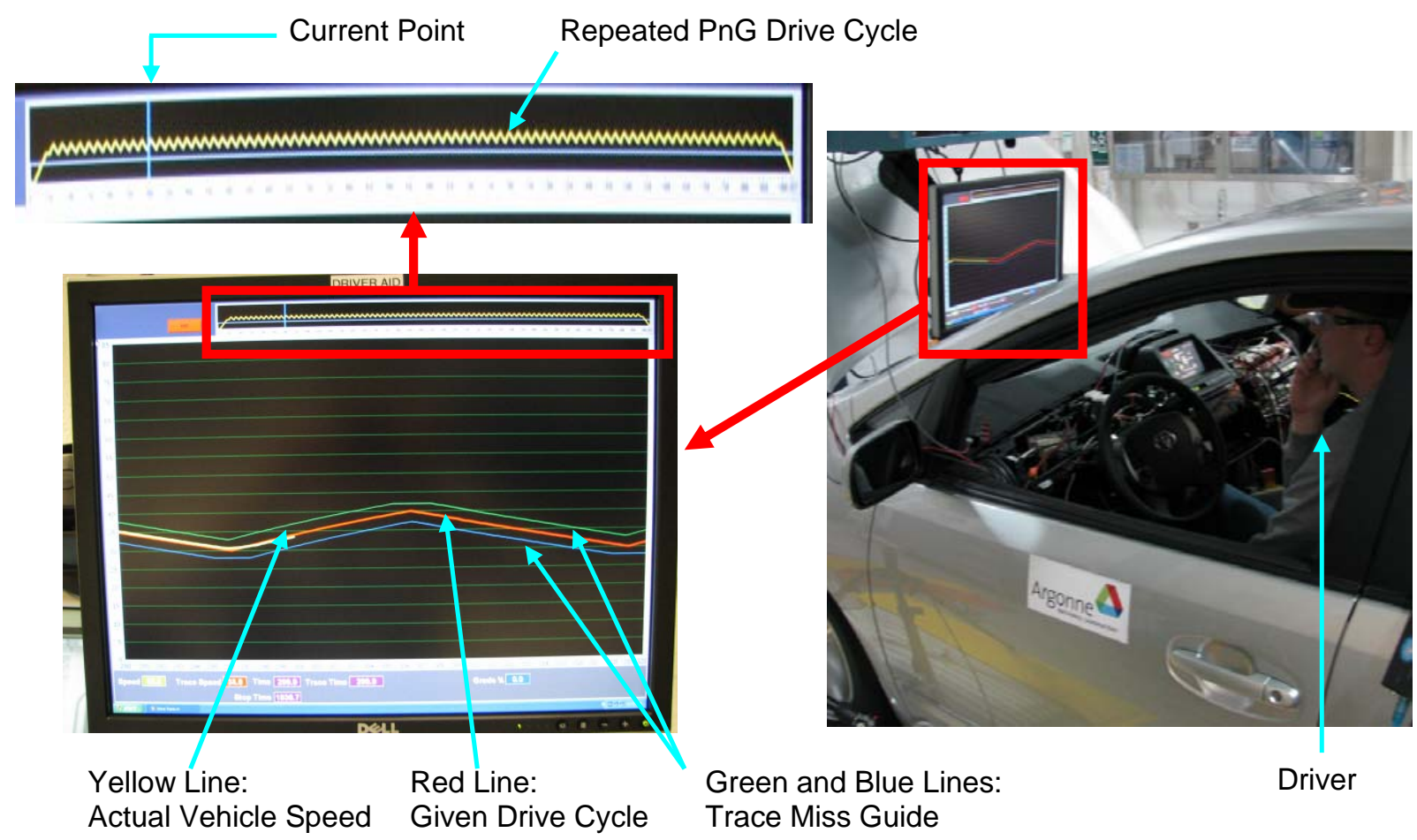

Figure 5.3 A picture of vehicle testing 
More detailed pictures of the test vehicle can be seen in Figure 5.3 above with brief descriptions. There is a screen in front of the windshield so a driver can see a drive cycle and control the accelerator pedal. In Figure 5.2, the red line on the screen represents the vehicle speed of the drive cycle, the green and blue lines indicate trace miss boundaries that the driver should keep the vehicle speed within those limits, and the yellow line shows the actual vehicle speed on the dynamometer. On the top of the screen, a whole drive cycle is displayed as well to let the driver know where the vehicle is on the drive cycle. In order to follow the given PnG drive cycles, a driver tries to keep the accelerator pedal position constant and it is one of convenient ways to generate relatively constant power at wheels without any additional power/torque controller. Note that the same road load coefficients (A, B and C) and vehicle test mass in Table 4.4 are used to set up the dynamometer road load and inertia for the HEV testing.

\subsection{Experimental Design: Selected Cases}

Before testing the HEV, experimental cases are designed to complete experiments in limited time due to ANL's testing schedule. At first, the same cases as PSAT simulation are considered for testing the HEV but there are 21 cases total; 18 cases of PnG driving and 3 cases of steady speed cases. However, it is very difficult to test all the cases in the given limited time so it is decided to reduce the cases. In addition, it is expected that the HEV has regenerative brake system and it can possibly affect fuel consumption of PnG driving strategy because the transmission (e-CVT) of the vehicle does not have a clutch as a conventional transmission. In other words, if a driver puts the gear position in $\mathrm{N}$ of the vehicle, the HEV system emulates that the engine, the motor and the gears are disengaged from each other because there is no conventional clutch in the planetary gear set and each gear is physically connected to each other. Therefore, it is necessary to focus further on those situations in the HEV testing.

As discussed in section 3.3.4, the higher deceleration in engine compression braking than in true coasting, does not help improving fuel economy for conventional vehicles. However, it can be interesting to apply that condition and investigate how the HEV system works in terms of fuel consumption. Also, slower deceleration rates than true coasting can affect fuel economy because the HEV uses the motor for EV mode when the driver's demand is relatively low at low speed. 
Finally, four different deceleration cases are selected as summarized in Table 5.2. However, only 10 seconds of acceleration is selected because this case shows the highest fuel economy improvement compared to steady speed cases by PSAT simulation in Chapter 4 . The same steady speed cases are also tested for comparison.

Table 5.2 Selected vehicle testing cases for the HEV, 2004 Toyota Prius

\begin{tabular}{|c|c|c|c|c|}
\hline \multicolumn{2}{|c|}{ Steady Speed Driving } & & & \\
\hline Test No. & Vehicle Speed & & & \\
\hline 1 & $25 \mathrm{mph}$ & & & \\
\hline 2 & $35 \mathrm{mph}$ & & & \\
\hline 3 & \multicolumn{2}{|l|}{$45 \mathrm{mph}$} & & \\
\hline \multicolumn{5}{|c|}{ PnG Driving } \\
\hline Test No. & $\begin{array}{l}\text { Vehicle } \\
\text { Speed } \\
\text { Range } \\
\end{array}$ & Pulse (Accel) & Glide (Decel) & Note for Glide Phase \\
\hline 4 & $20-30 \mathrm{mph}$ & \multirow{3}{*}{$\begin{array}{l}10 \text { seconds } \\
\text { of acceleration }\end{array}$} & \multirow{3}{*}{$\begin{array}{c}\text { Coast Down } \\
\text { Gear in N } \\
\text { Accelerator pedal position: } \\
0 \%\end{array}$} & \multirow{3}{*}{$\begin{array}{l}\text { - Zero wheel torque from HEV } \\
\text { - Zero battery current except } \\
\text { accessory loads }\end{array}$} \\
\hline 5 & $30-40 \mathrm{mph}$ & & & \\
\hline 6 & $40-50 \mathrm{mph}$ & & & \\
\hline 7 & $20-30 \mathrm{mph}$ & \multirow{3}{*}{$\begin{array}{l}10 \text { seconds } \\
\text { of acceleration }\end{array}$} & \multirow{3}{*}{$\begin{array}{c}\text { Deceleration } \\
\text { Gear in } D \\
\text { Accelerator pedal position: } \\
0 \%\end{array}$} & \multirow{3}{*}{$\begin{array}{l}\text { - HEV emulates engine compression } \\
\text { braking } \rightarrow \text { mild regen comes in }\end{array}$} \\
\hline 8 & $30-40 \mathrm{mph}$ & & & \\
\hline 9 & $40-50 \mathrm{mph}$ & & & \\
\hline 10 & $20-30 \mathrm{mph}$ & \multirow{3}{*}{$\begin{array}{l}10 \text { seconds } \\
\text { of acceleration }\end{array}$} & \multirow{3}{*}{$\begin{array}{c}\text { Same Deceleration Rate as } \\
\text { Coast Down } \\
\text { Powered Deceleration* } \\
\text { Gear in D } \\
\text { Accelerator pedal position: } \\
>0 \%\end{array}$} & \multirow{3}{*}{$\begin{array}{l}\text { - Push accelerator pedal position until } \\
\text { HEV emulates true coasting } \\
\rightarrow \text { same travel distance as true } \\
\text { Coast down but battery discharges }\end{array}$} \\
\hline 11 & $30-40 \mathrm{mph}$ & & & \\
\hline 12 & $40-50 \mathrm{mph}$ & & & \\
\hline 13 & $20-30 \mathrm{mph}$ & \multirow{3}{*}{$\begin{array}{l}10 \text { seconds } \\
\text { of acceleration }\end{array}$} & \multirow{3}{*}{$\begin{array}{c}\mathbf{0 . 6} * \text { Deceleration Rate of } \\
\text { Coast Down } \\
\text { Powered Deceleration* } \\
\text { Gear in D } \\
\text { Accelerator pedal position: } \\
\quad>0 \%\end{array}$} & \multirow{3}{*}{$\begin{array}{l}\text { - Battery discharge (EV mode) } \\
\rightarrow \text { longer travel distance but } \\
\text { battery discharges }\end{array}$} \\
\hline 14 & $30-40 \mathrm{mph}$ & & & \\
\hline 15 & $40-50 \mathrm{mph}$ & & & \\
\hline
\end{tabular}

* Powered deceleration: The engine or the motor produces positive output power but the vehicle is still in deceleration because the output power is not enough to accelerate the vehicle.

First, the tests 1, 2 and 3 are for steady speed driving cases at $25 \mathrm{mph}, 35 \mathrm{mph}$ and $45 \mathrm{mph}$, respectively. The tests 4,5 and 6 are for true coast down which means the gear is in $\mathrm{N}$ and the accelerator pedal position is at $0 \%$. In this case, the wheel torque is zero and the only output 
battery power is for accessory loads. The next case for tests 7, 8 and 9 is decelerating the vehicle with gear in drive (D) but the accelerator pedal position is still same at zero. The HEV system emulates engine compression braking in this condition using mild regenerative braking. The tests 10, 11 and 12 use the same deceleration rate (same vehicle speed profile) of tests 4,5 and 6 but the gear should be in D and the driver has to push the accelerator pedal a little bit to follow the vehicle speed to produce enough power by either the engine or the motor. This condition is called 'powered deceleration.' The last test set is another powered deceleration cases with slower rates, so the vehicle is in longest deceleration among other cases. For this case, the driver should push the accelerator pedal even deeper than the previous case to follow the vehicle speed. Note that the overall time for each case is approximately 20 minutes. The vehicle speed profiles of each case in the $20-30 \mathrm{mph}$ speed range can be seen in Figure 5.4 below. The speed profile for emulated compression braking cases is obtained from preliminary vehicle testing of the HEV at ANL. Also, the speed profile for the slower deceleration rate cases (powered deceleration) is generated by multiplying 0.6 to the deceleration rate of the coast down cases.

Different Vehicle Speed Profiles for Testing 2004 Toyota Prius

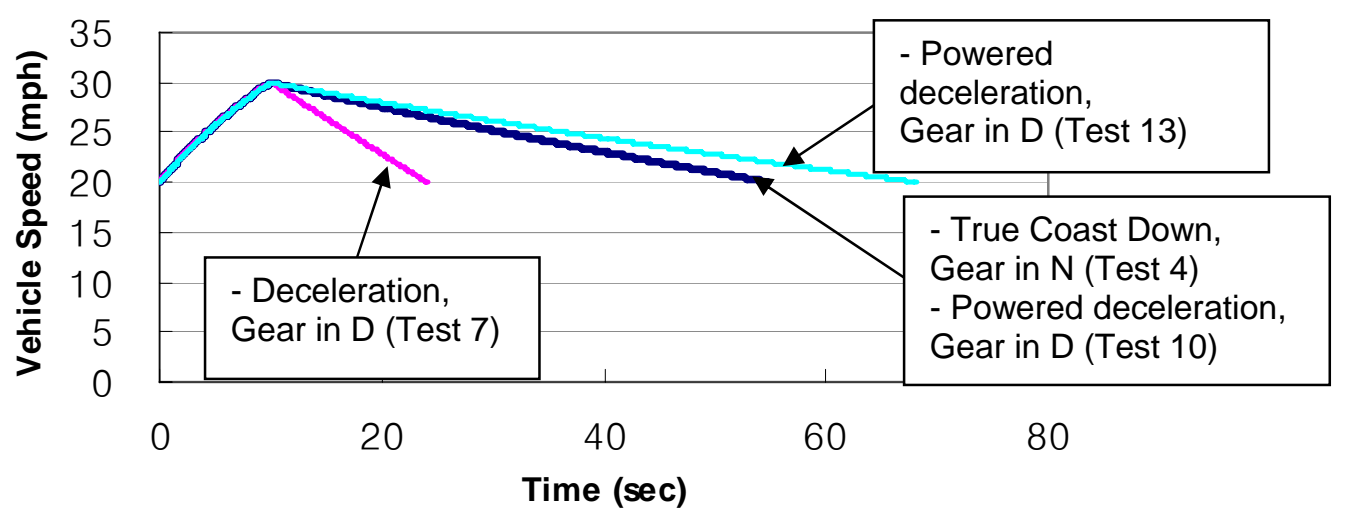

- Tests $4 \& 10$ - Test 7 - Test 13

Figure 5.4 Different deceleration rates for vehicle testing, 20 - $30 \mathrm{mph}$ of $\mathrm{PnG}$ driving

In Chapter 5, experimental set up for the HEV testing and experimental cases are designed to investigate the impact of the PnG driving strategy on fuel consumption compared to the steady speed driving. In the vehicle testing, the deceleration rate in the coast phase is more focused because the HEV is equipped with an additional power source (a motor and battery) rather than 
an engine and an unconventional transmission so it has more capabilities to alter fuel consumption of the PnG driving strategies, for example, EV mode and regenerative braking. The following chapter will discuss the results from the HEV testing in detail. 


\section{Chapter 6 Experimental Results and Discussion}

The vehicle testing for the HEV described in Chapter 5 was conducted at ANL to investigate the impact of the PnG driving strategy on fuel consumption. In this chapter, the results of the selected PnG drive cycles are analyzed and discussed in detail. As previously mentioned, HEVs have more complicated systems than conventional vehicles so the results should be carefully analyzed. The vehicle testing results are not only compared with one of the HEV simulation results (10 seconds of acceleration) in Chapter 4 but also compared with each other for the impact of the deceleration rates on fuel consumption. The SOC of the battery, the EV mode and the efficiency of KE storage are discussed as well. Again, the vehicle specifications of the HEV are the same as shown in Table 4.4. In short, the results show that the fuel consumption of the PnG cases (tests 4, 5, and 6) with the gear in $\mathrm{N}$ position (true coast down in glide phase) is worse than that of the steady speed driving. However, some of the other PnG cases show fuel economy improvement compared to each steady speed driving. More details are presented in the following sections.

\subsection{Fuel Rate and SOC of Battery}

The fuel rate from the vehicle testing is analyzed first to investigate how the engine in the HEV is operating in the PnG drive cycles. The results show different fuel rate patterns compared to the simulation results for the HEV, especially for the real PnG cases with true coast down. First, the fuel rate of the steady speed cases in a certain period is plotted in Figure 6.1 below. 
Fuel Rate, Vehicle Speed, Battery Power and SOC for Steady Speed, 2004 Toyota Prius

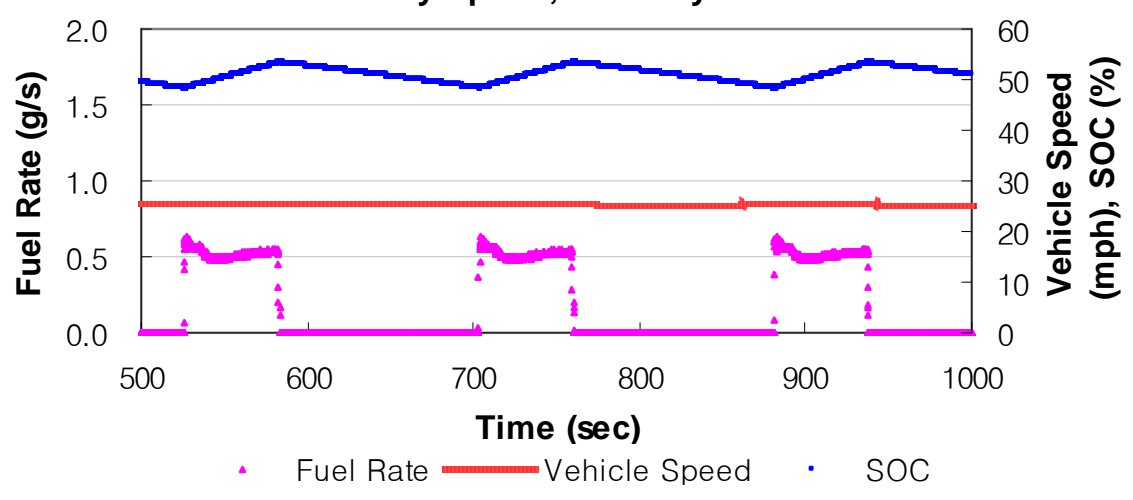

(a) Test 1: Steady speed at $25 \mathrm{mph}$

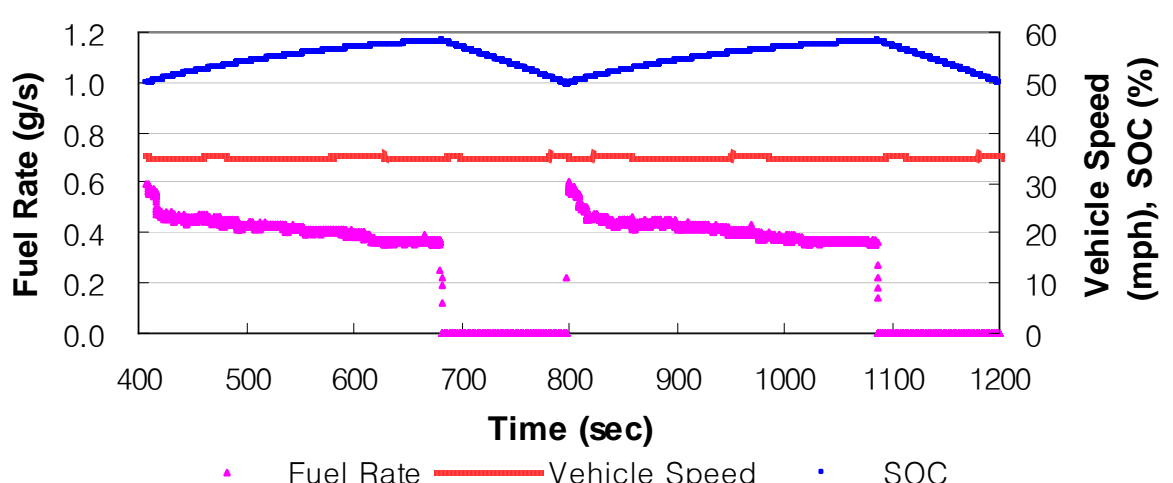

(b) Test 2: Steady speed at $35 \mathrm{mph}$

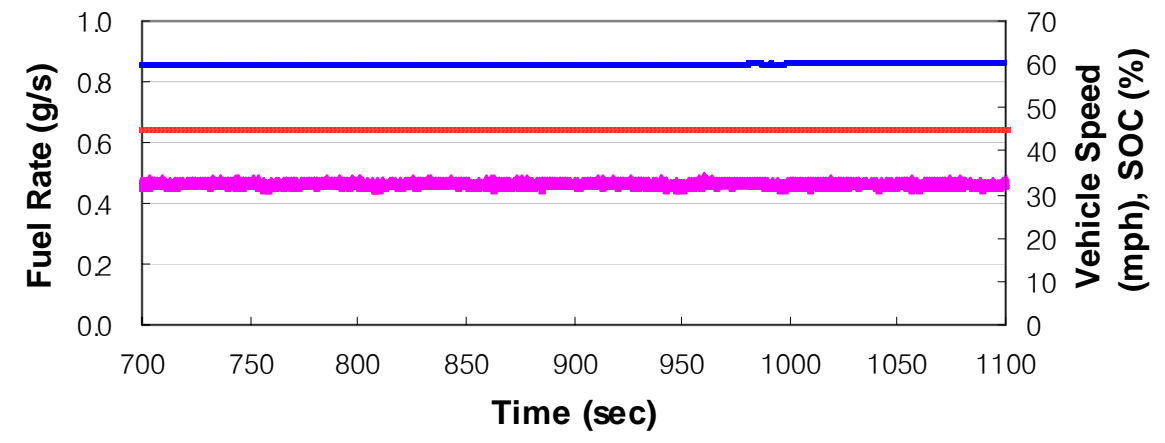

- Fuel Rate $\longrightarrow$ Vehicle Speed $\quad$ SOC

(c) Test 3: Steady speed at $45 \mathrm{mph}$

Figure 6.1 Fuel rate and SOC of battery in the steady speed driving from vehicle testing, 2004 Toyota Prius 
In steady speed driving, the HEV shows different fuel consumption pattern rather than a conventional vehicle. In most conventional vehicles with internal combustion engines, the fuel rate is constant if the gear is not shifted and the road conditions are not changed. However, the HEV has a battery so it can use the battery power when it is necessary. As shown in Figure $6.1-$ (a), the fuel rate is not constant and sometimes it is zero which means the engine is on and off. When the engine is on, it produces enough power to maintain the speed and charges the battery with extra power so the SOC is increased. In contrast to that, the vehicle uses the battery power only to keep the vehicle speed at $25 \mathrm{mph}$ when the engine is off. Hence, the SOC is decreased in this interval. The similar fuel rate pattern can be seen in Figure 6.1 - (b) for the $35 \mathrm{mph}$ of steady speed case. However, it shows much shorter period of the EV mode compared to the 25 mph case because the road load power due to aerodynamic drag and rolling resistance is increased by the higher steady speed and the vehicle needs more power from the engine rather than the battery. The battery capacity cannot provide enough power for a long time at higher speed. In short, the HEV uses electric power only at relatively lower steady speeds. On the contrary, the $45 \mathrm{mph}$ steady speed shows that there is no EV mode and the engine is always operating. In this case, the SOC and the fuel rate are almost constant over the drive cycle. In other words, the HEV behaves as conventional vehicles when it is at relatively higher steady speeds.

The engine load leveling for steady speed cases in vehicle testing discussed above can be explained with respect to power levels. As described in few references [33][35], the engine follows the optimized operation line in a 2004 Toyota Prius and the engine efficiency is plotted in Figure 6.2 along with engine power. At each steady speed, the HEV needs a certain level of tractive power, $1.7 \mathrm{~kW}$ at $25 \mathrm{mph}, 3.1 \mathrm{~kW}$ at $35 \mathrm{mph}$, and $5.3 \mathrm{~kW}$ at $45 \mathrm{mph}$ respectively. As shown in Figure 6.2 - (a), if the engine power is below $2 \mathrm{~kW}$, the engine efficiency is lower than $20 \%$. Thus, the HEV system does engine load leveling to charge the battery in high efficiency region and uses the battery electric energy when the tractive power is low. Therefore, the steady speed cases at $25 \mathrm{mph}$ and $35 \mathrm{mph}$ show the fuel rate and SOC of the battery in vehicle testing in Figures 6.1 - (a) and (b). However, the tractive power at $45 \mathrm{mph}$ is $5.3 \mathrm{~kW}$ and the engine efficiency is approximately $30 \%$, so the vehicle does not do engine load leveling as shown in Figure $6.1-(\mathrm{c})$. Also, the engine must be spinning above $40 \mathrm{mph}$ due to e-CVT speed limit. 
The engine load leveling at low speed is as efficient as the PnG driving strategy because fuel economy is improved significantly as explained in later sections.

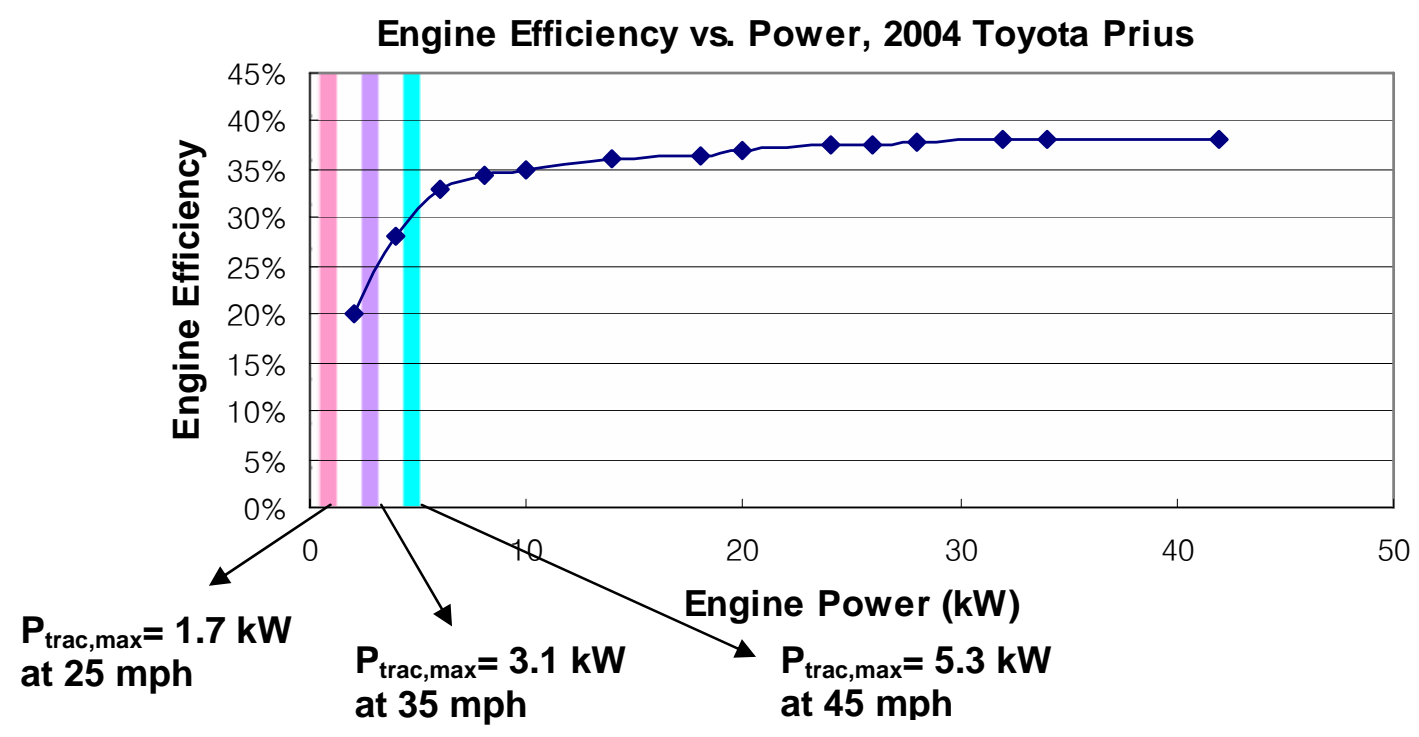

(a) Steady speed cases

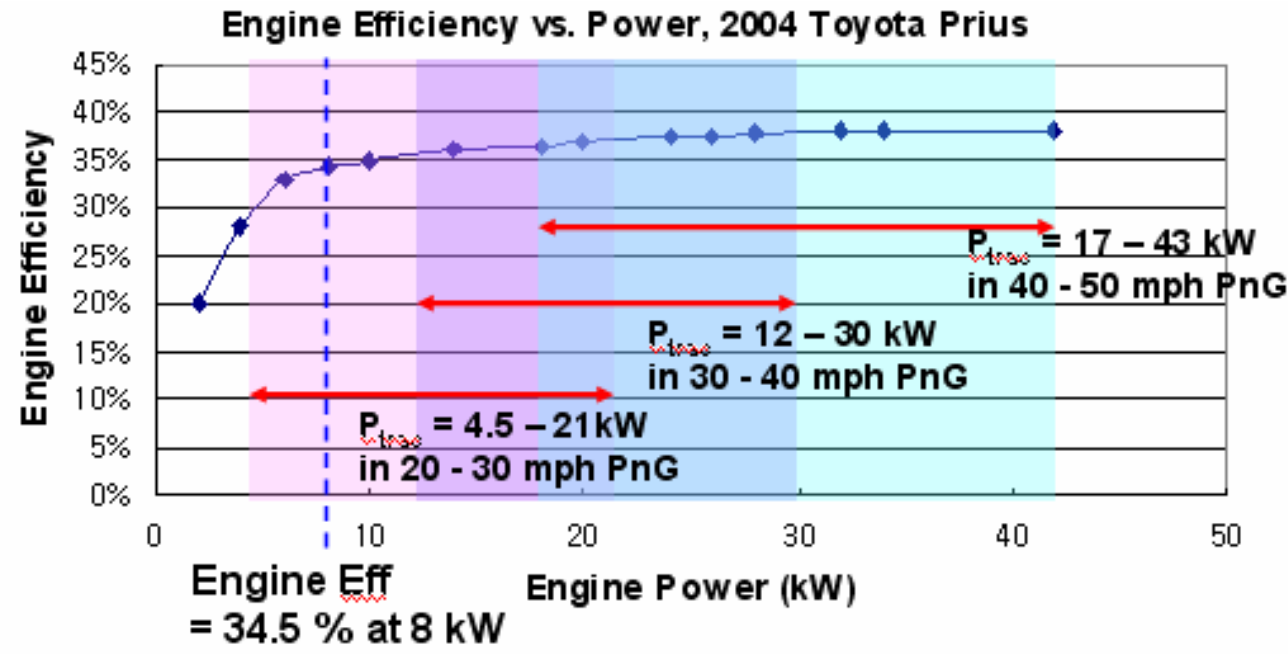

(b) PnG cases

Figure 6.2 Engine efficiency and engine power for optimized engine operating line of 2004 Toyota Prius

In the PnG driving, the tractive power is increased due to acceleration as shown in Figure $6.2-$ (b). The tractive power for the PnG cases is $4.5-21 \mathrm{~kW}$ in $20-30 \mathrm{mph}, 12-30 \mathrm{~kW}$ in $30-40$ $\mathrm{mph}$, and $17-43 \mathrm{~kW}$ in $40-50 \mathrm{mph}$ respectively from 5 seconds to 30 seconds of acceleration. 
As shown in Figure 6.2, the engine efficiency cannot be increased dramatically as the engine power increases to the level of tractive power for the PnG cases once it reaches to a high level $(34.5 \%$ at $8 \mathrm{~kW})$. Also, HEVs have battery power limit ( $22 \mathrm{~kW}$ for the test vehicle) for charge and discharge, hence hybrid electric engine load leveling has limitations in the PnG driving. In contrast to that, storing $\mathrm{KE}$ in vehicle inertia by the PnG driving can improve fuel economy further because there is no battery power limit.

Figure 6.3 shows the PnG cases in true coast down with gear in $\mathrm{N}$ position as explained in Chapter 4. During vehicle testing for those cases, the test driver puts the gear in N position while the vehicle is in glide phase and it represents true PnG driving strategies. Again, the acceleration time for PnG driving cases is fixed to 10 seconds in vehicle testing. As shown in the figures, the fuel is still consumed during coasting rather than zero as expected from the simulation results in Chapter 4. Also, the SOC during coasting does not increase at all and it means that the battery is not charged by the engine. The regenerative braking is not applied because the gear is set to $\mathrm{N}$ and the driver does not push the brake pedal during the coasting period. It seems like the HEV is set to be very similar to conventional vehicles. 
Fuel Rate, Vehicle Speed, Battery Power and SOC for

PnG: Tests 4, 5 and 6, 2004 Toyota Prius

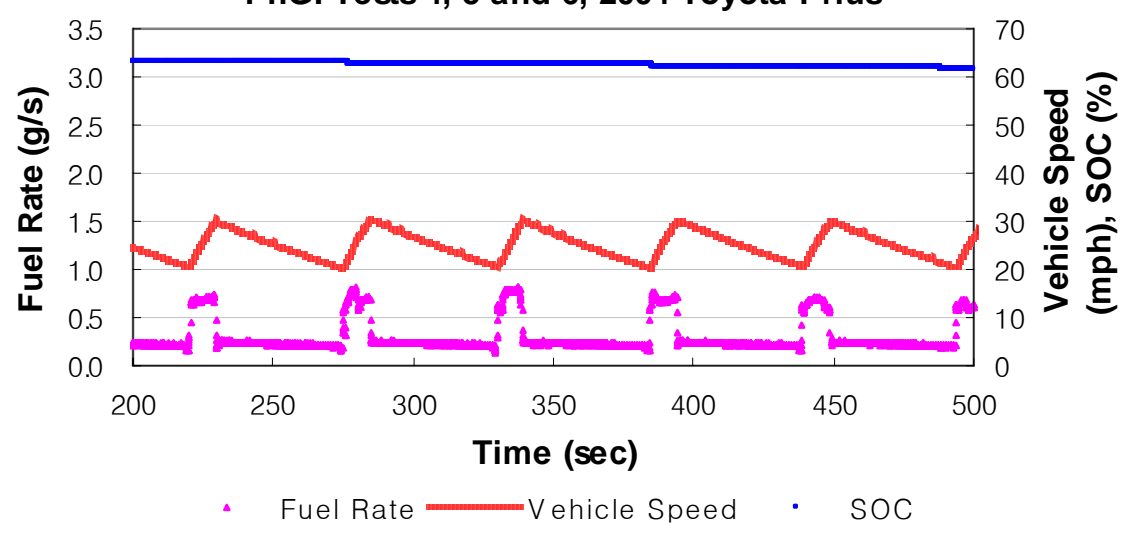

(a) Test 4: PnG in $20-30 \mathrm{mph}$

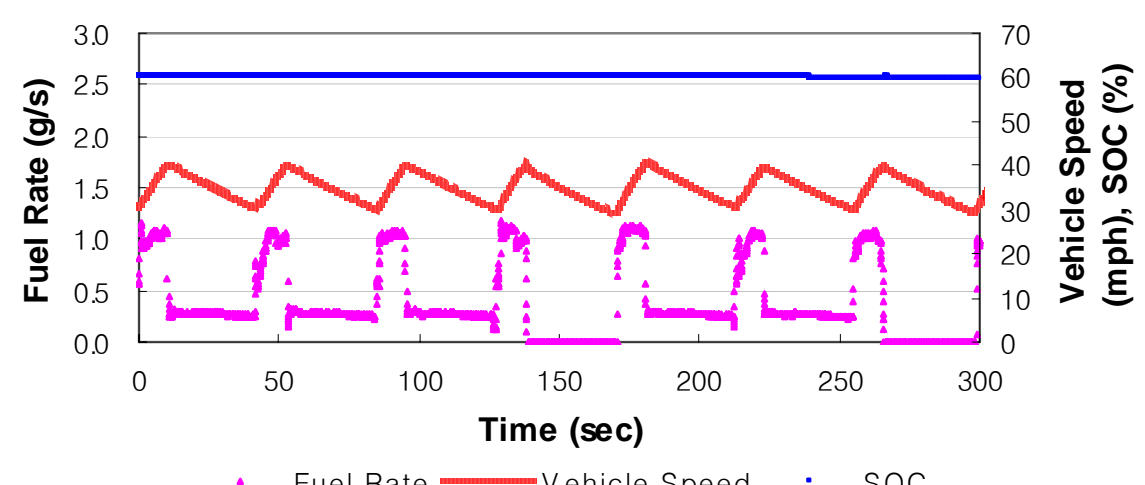

(b) Test 5: PnG in $30-40 \mathrm{mph}$

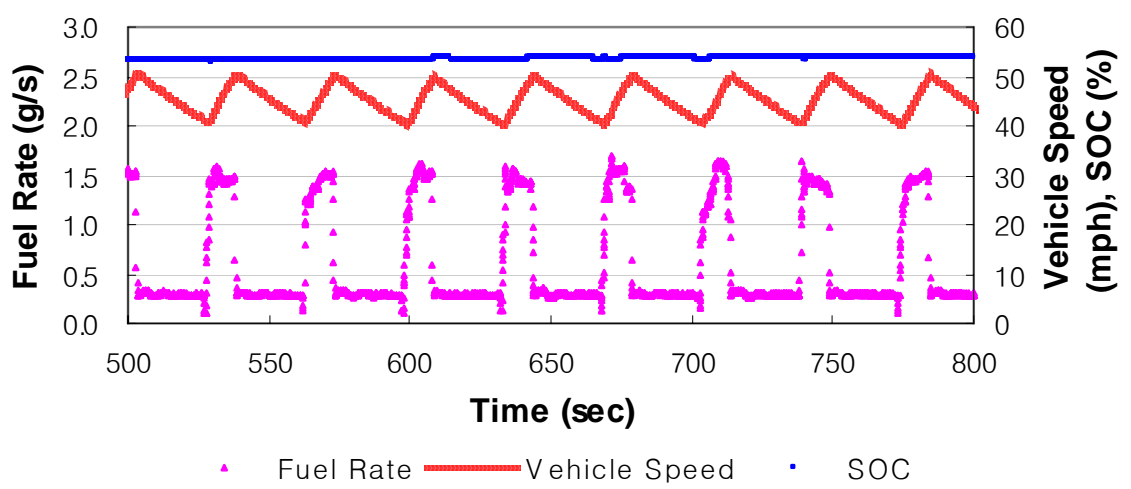

(c) Test 6: PnG in $40-50 \mathrm{mph}$

Figure 6.3 Fuel rate and SOC of battery in the PnG driving in true coast down with gear in $\mathrm{N}$ position from vehicle testing, 2004 Toyota Prius 
If the gear is in $\mathrm{N}$ position in a conventional vehicle without any driver's demand, the engine is idling and consumes fuel to keep the engine operating due to safety issues. In short, the engine consumes fuel during coasting but it neither charges the battery nor produces power to propel the vehicle. It is clearly shown in Figures 6.4 and 6.5 below.

Figure 6.4 shows the battery power with the engine fuel rate in the HEV during PnG driving in $30-40 \mathrm{mph}$ in true coast down and gear in $\mathrm{N}$ (test 5 as shown in Figure $6.3-$ (b) above). The battery power is very small $(<0.5 \mathrm{~kW})$ during coasting and it is assumed to be accessory load. It can also be regarded as positive power to propel the vehicle during coasting (powered deceleration) because the planetary gear set in the HEV does not have a clutch and cannot be disengaged physically. However, the planetary gear set does not produce any positive power to propel the vehicle during coasting as shown in Figure 6.5 below. The power from the ring gear represents the output power from the HEV system, so if it is positive, it means that the vehicle produces power to propel the vehicle, and if it is negative, it means that the vehicle uses regenerative braking to slow down. In addition, the sun gear is connected to the generator and it does not produce any electric power to charge the battery in this case. If the output power of the sun gear is positive, it means that the generator is either charging the battery or there is power circulation from the generator to the motor. In summary, the engine fuel consumption during coasting for the PnG case is not for any useful work but it might be for safety issues. Note that the power from each gear is calculated from equations in Appendix A

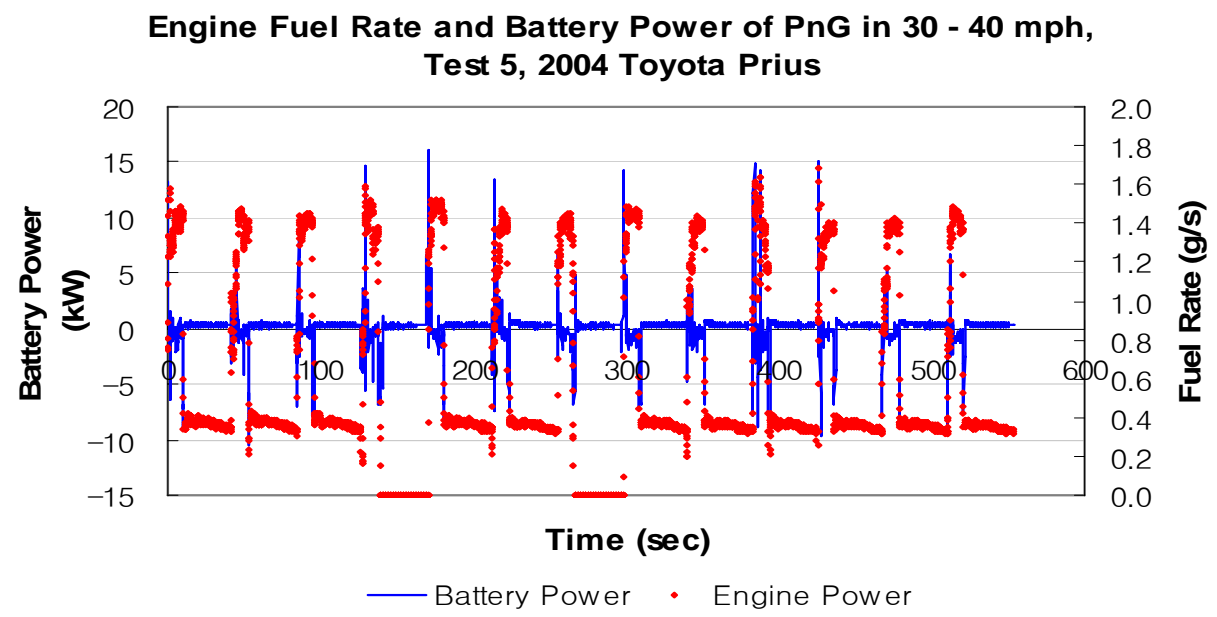

Figure 6.4 Engine fuel rate and battery power of the PnG driving in $30-40 \mathrm{mph}$ from vehicle testing (test 5), 2004 Toyota Prius 


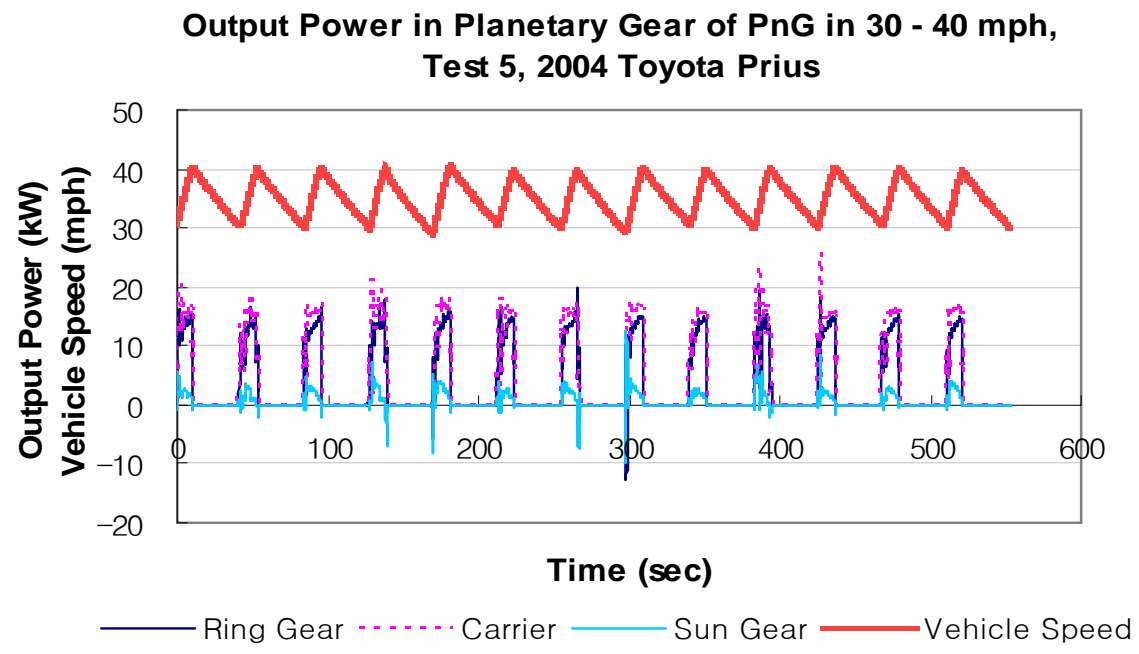

Figure 6.5 Output power in planetary gear of the PnG driving in $30-40 \mathrm{mph}$ from vehicle testing (test 5), 2004 Toyota Prius

The fuel consumption during coasting which is shown in Figure $6.3-$ (a) is not present in the simulation results in Figure 4.12 and results in more fuel consumption over the PnG drive cycles. The fuel economy of these cases is much lower than that of the steady speed driving cases as described later in section 6.4.2.

From unexpected results (tests 4, 5, and 6) for the true PnG driving strategy on the HEV as discussed above, the vehicle is tested for the same PnG drive cycles but with gear in $\mathrm{D}$. In order to follow the same vehicle speed profiles, the driver pushes the accelerator pedal slightly. The fuel rate and the SOC are plotted with vehicle speed in Figure 6.6 below. All the PnG cases in Figure 6.6 show that the engine consumes a certain amount of fuel in acceleration but the engine is on and off depending on the SOC in deceleration (coast phase). In these PnG cases, the HEV shows all three cases; the engine operating mode, the EV mode and the transient mode. For example, in low speed range, $20-30 \mathrm{mph}$, the engine consumes fuel to charge the battery if the SOC is relatively low during deceleration as seen in Figure $6.6-$ (a). However, once the SOC is at a certain level, the vehicle may be in EV mode or in transient mode operated by either the engine or the motor. This trend can be seen in higher speed ranges, $30-40 \mathrm{mph}$ in Figure $6.6-$ (b) and $40-50 \mathrm{mph}$ in Figure 6.6 - (c) respectively. In short, the engine consumes less fuel in these PnG cases compared to the PnG cases with true coast down because the engine can be off with gear in $\mathrm{D}$ and the vehicle can use electric power during deceleration. 
Fuel Rate, Vehicle Speed, Battery Power and SOC for PnG: Tests 10, 11 and 12, 2004 Toyota Prius

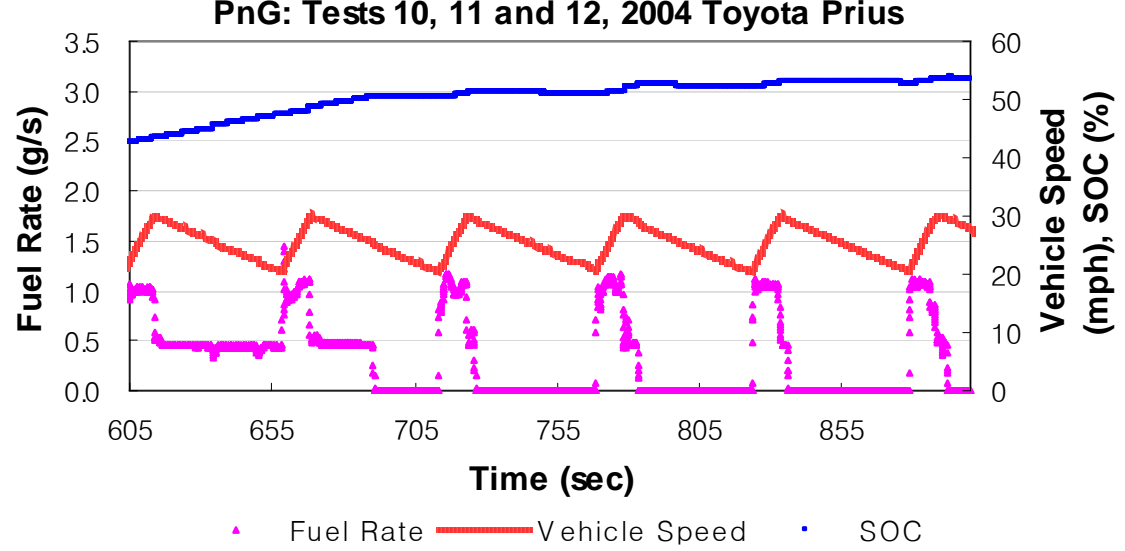

(a) Test 10: PnG in $20-30 \mathrm{mph}$

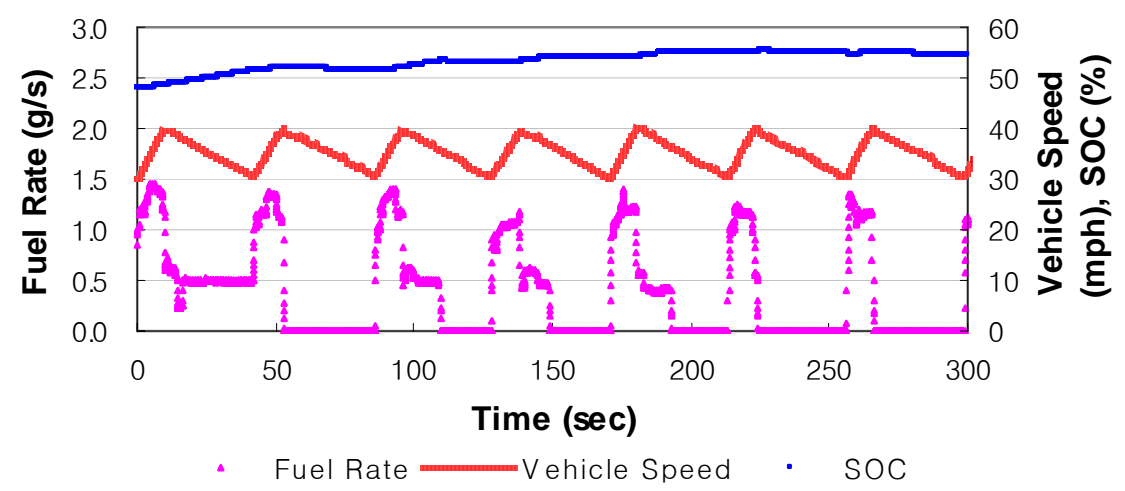

(b) Test 11: PnG in $30-40 \mathrm{mph}$

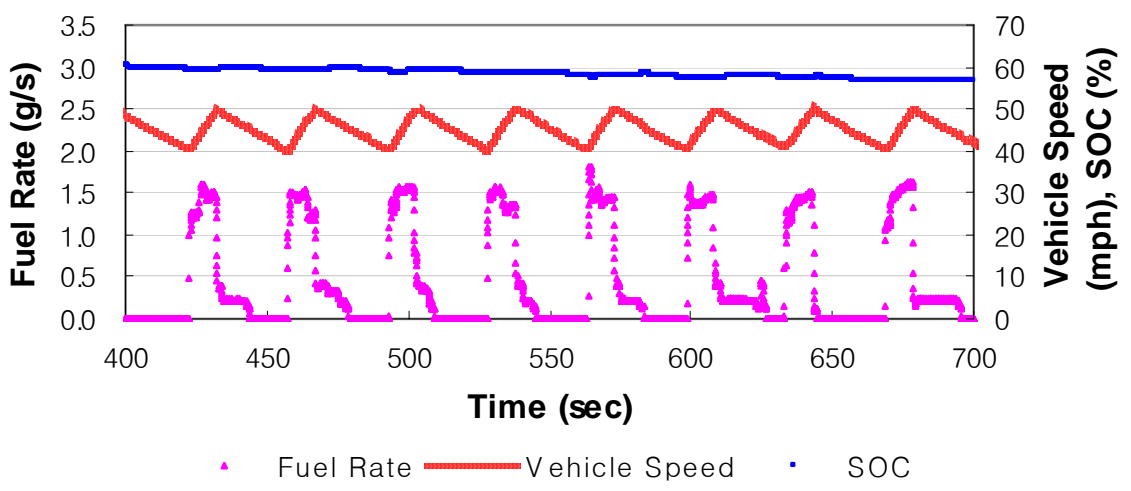

(c) Test 12: PnG in $40-50 \mathrm{mph}$

Figure 6.6 Fuel rate and SOC of battery in the PnG driving with the same drive cycle as the PnG with true coast down and gear in D position from vehicle testing, 2004 Toyota Prius 
Fuel Rate, Vehicle Speed, Battery Power and SOC for PnG: Tests 7, 8 and 9, 2004 Toyota Prius

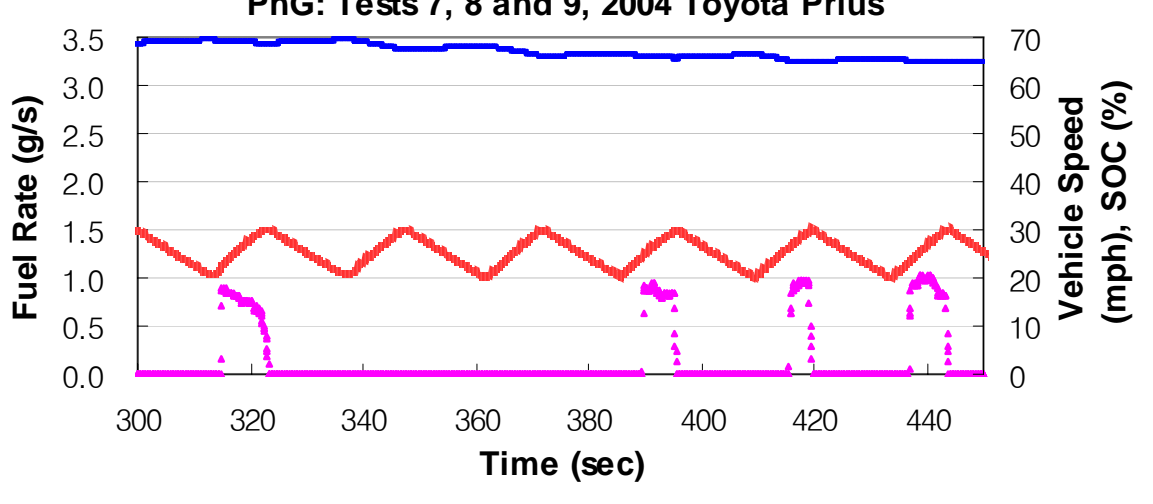

- Fuel Rate manicle Speed - SOC

(a) Test 7: PnG in $20-30 \mathrm{mph}$

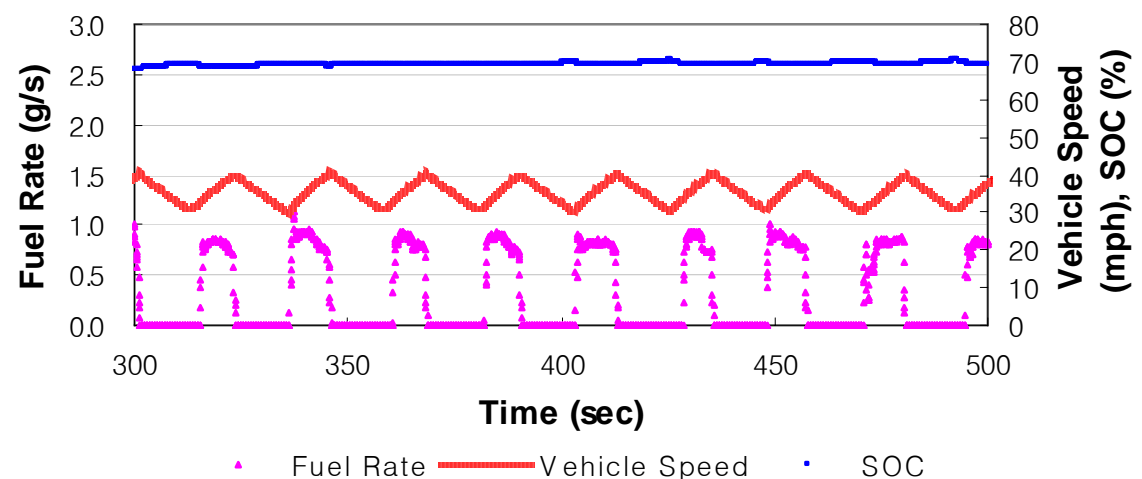

(b) Test 8: PnG in $30-40 \mathrm{mph}$

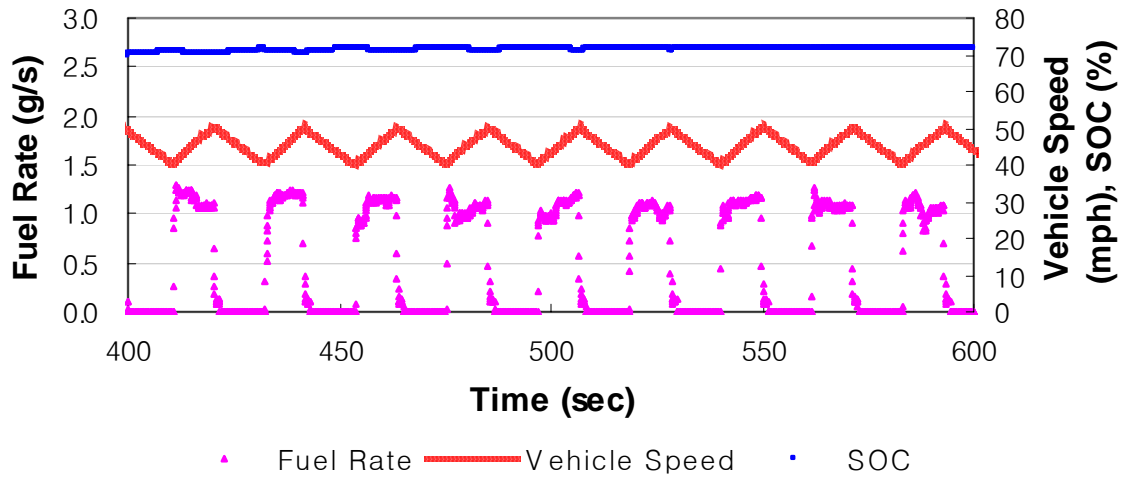

(c) Test 9: PnG in $40-50 \mathrm{mph}$

Figure 6.7 Fuel rate and SOC of battery in the PnG driving with no driver's demand and gear in D position from vehicle testing, 2004 Toyota Prius 
As briefly mentioned in section 5.2, the emulated engine compression braking of the HEV can improve the fuel economy with the PnG driving strategy because it emulates engine compression braking and charges the battery using mild regenerative braking. For these PnG cases, the driver puts the gear in D position and does not push the accelerator (or brake) pedal at all. It other words, the only difference between true PnG cases and these cases is the gear position in the view of the driver. It makes the deceleration time shorter in the same speed range. Again, Figure 6.7 above shows the fuel rate and the SOC with vehicle speed. In low speed range, 20 $30 \mathrm{mph}$, the engine is sometimes off during acceleration because the SOC is high enough to propel the vehicle with electric power only. However, the engine is on for the most of time during acceleration in higher speed ranges (see Figure $6.7-(b)$ and Figure $6.7-(c)$ ). During deceleration, the engine is completely off because there is no driver's demand and the regenerative brake system charges the battery. The higher speed range cases also show complete engine off during deceleration. Note that the slight increase of the SOC during deceleration can be seen, especially in Figure 6.7 - (a). In those PnG cases, the fuel consumption can be reduced but the VMT is also shorter so it can affect the benefit of fuel economy by the PnG. The fuel economy results for these cases are discussed in section 6.4.2 as well.

The last PnG cases are for slower deceleration rates during coasting as shown in Figure 6.8 below. Note that the slower deceleration rates are obtained by multiplying the deceleration rates of true coast down of the HEV by 0.6. The deceleration time is longer than any other PnG cases due to the slower deceleration rates. In theses case, the driver should push the accelerator pedal to make the powered deceleration condition during deceleration. As plotted in Figure $6.8-(\mathrm{a})$, the engine is off during deceleration when the SOC is decreased because the vehicle is in EV mode. However, the engine sometimes consumes fuel to charge the battery and the SOC is increased. The SOC goes up and down over the drive cycle. A similar trend is shown in the 30 - $40 \mathrm{mph}$ case in Figure 6.8 - (b). However, the engine is on most of the time during deceleration in $40-50 \mathrm{mph}$ of $\mathrm{PnG}$ driving. The SOC does not change a lot in this case compared to the lower speed range cases so it means that the engine generates power to slow the deceleration rate and slightly charge the battery with extra power. 
Fuel Rate, Vehicle Speed, Battery Power and SOC for PnG: Tests 13, 14 and 15, 2004 Toyota Prius

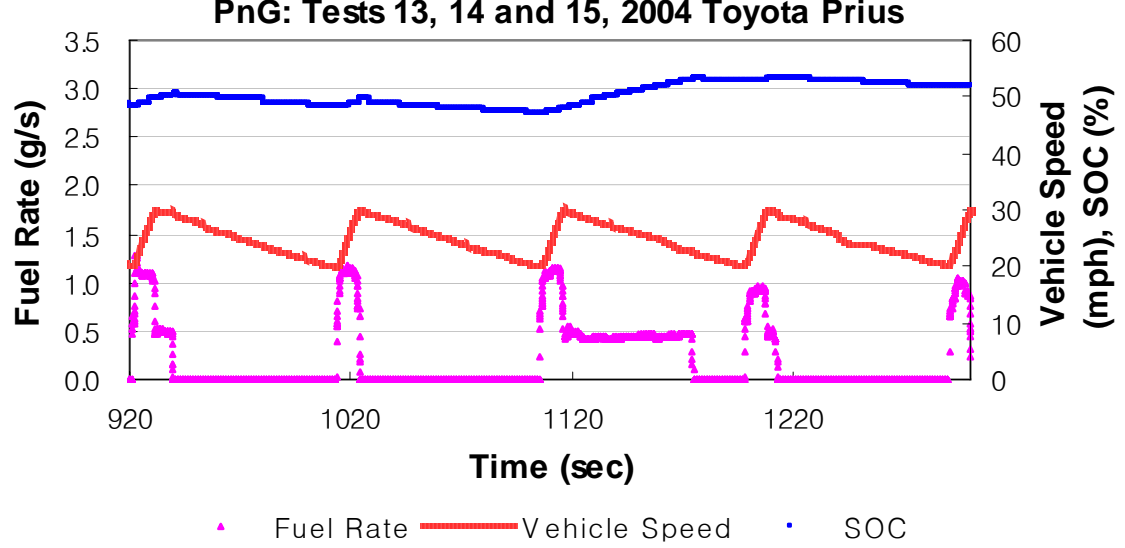

(a) Test 13: PnG in $20-30 \mathrm{mph}$

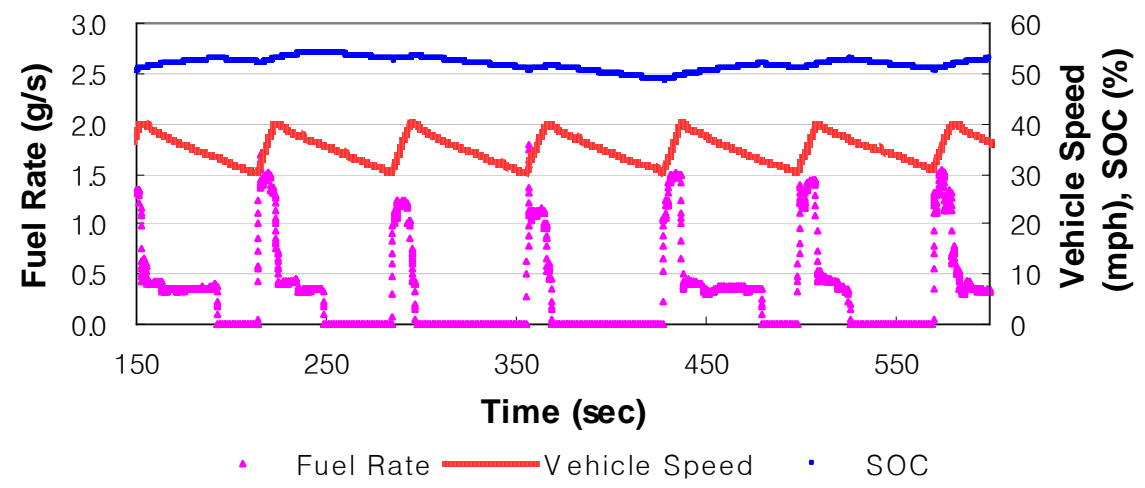

(b) Test 14: PnG in $30-40 \mathrm{mph}$

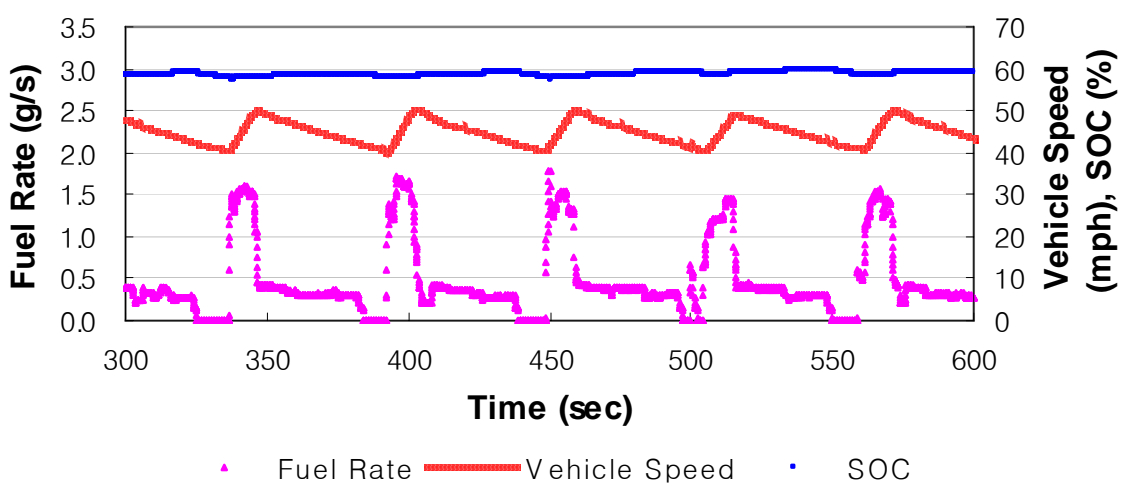

(c) Test 15: PnG in $40-50 \mathrm{mph}$

Figure 6.8 Fuel rate and SOC of battery in PnG driving with the same drive cycle as the PnG with slower deceleration and gear in D position from vehicle testing, 2004 Toyota Prius 
In summary, the trend of fuel rate and SOC for various PnG driving cases is shown in this section. The engine in the HEV consumes fuel to propel the vehicle and charge the battery. Also, the HEV is occasionally in EV mode or in engine operation mode depending on the driver's demand over the PnG drive cycles. The fuel and the SOC discussed in this section cannot directly represent the benefit of fuel economy by the PnG driving strategy, but they show how the HEV behaves for the given drive cycles compared to the steady speed driving conditions. More details will be analyzed and described in the following section.

\subsection{Cumulative Fuel Use}

The cumulative fuel use is obtained by summation of the fuel rate over the drive cycles. Figures 6.8, 6.9 and 6.10 show the first 400 seconds of cumulative fuel use of each steady speed and PnG driving case respectively. If absolute values of the cumulative fuel use in the figures are even higher than that of the other cases, it does not mean that the case has higher fuel economy as discussed for the conventional vehicle. The steady speed and PnG cases discussed for the conventional vehicle in Chapter 4 are not for repeated drive cycles but for one complete cycle so they can be easily compared to each other in terms of absolute values of cumulative fuel use. However, all drive cycles for the HEV have different lengths and the VMT for each case varies. Therefore, the cumulative fuel use plotted over the time here should be divided by distance and considered with SOC balance for accurate comparison of fuel economy.

In Figure 6.9, the cumulative fuel use of the steady speed driving does not show a linear increase as shown in Figure 4.1 from the simulation results. It is similar to the PnG cases because the engine is on and off in steady speed at $25 \mathrm{mph}$ as described in Figure $6.1-$ (a). This result is not present in the PSAT simulations, indicating that some of the details of the HEV control strategy are not well represented in the simulation model. Also, the PnG case with true coast down and gear $\mathrm{N}$ (test 4) shows almost linear increase of fuel consumption because the engine consumes fuel even during coasting as discussed previously. The cumulative fuel use shows stepwise patterns for the other PnG cases in $20-30$ mph range. 


\section{Cumulative Fuel Use for Png in 20 - 30 mph and Steady Speed at 25 mph, 2004 Toyota Prius}

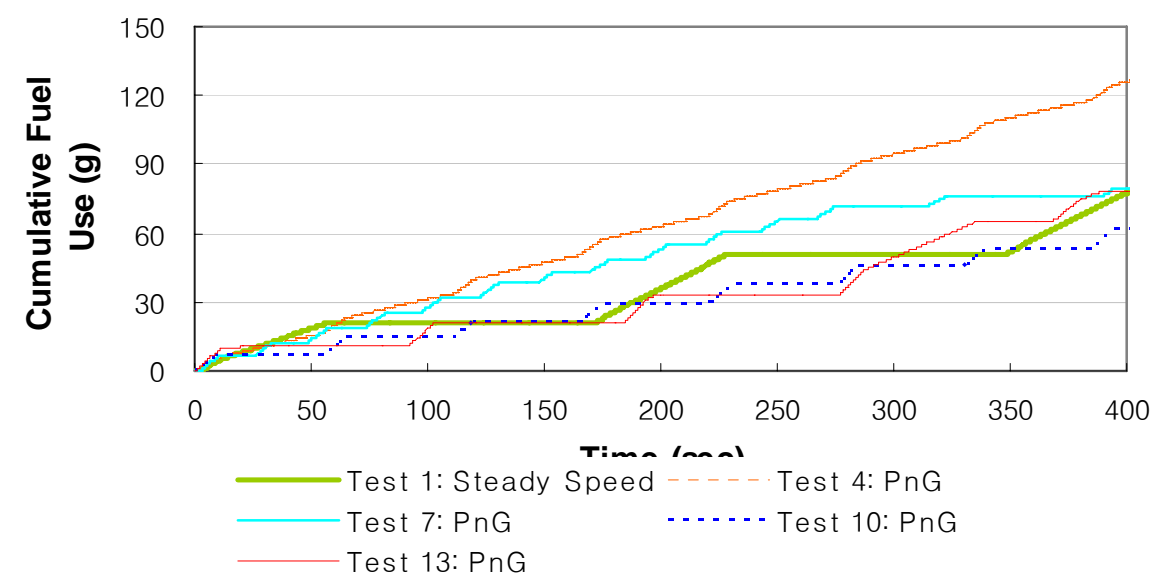

Figure 6.9 Cumulative fuel use comparison for PnG in $20-30 \mathrm{mph}$ and steady speed at $25 \mathrm{mph}$, 2004 Toyota Prius

At $35 \mathrm{mph}$ steady speed, the fuel consumption shows a similar trend to the $25 \mathrm{mph}$ steady speed case but it is more stretched because the engine off period is shorter. All PnG cases show stepwise patterns in fuel consumption. In contrast to that, the $45 \mathrm{mph}$ steady speed case has a linear increase of fuel consumption in Figure 6.11 because the engine is always on and consumes fuel at $45 \mathrm{mph}$ steady speed. Again, the other PnG cases show similar patterns of cumulative fuel consumption.

In the HEV, the trend of the cumulative fuel use is a little different from the simulation results, especially, the steady speed driving cases at $25 \mathrm{mph}$ and $35 \mathrm{mph}$. It can be assumed that PSAT is accurate to get fuel economy for general drive cycles but it has some discrepancy for this kind of particular PnG drive cycles because HEVs have very complex systems and control strategies. 


\section{Cumulative Fuel Use for PnG in 30 - $40 \mathrm{mph}$ and Steady Speed at $35 \mathrm{mph}, 2004$ Toyota Prius}

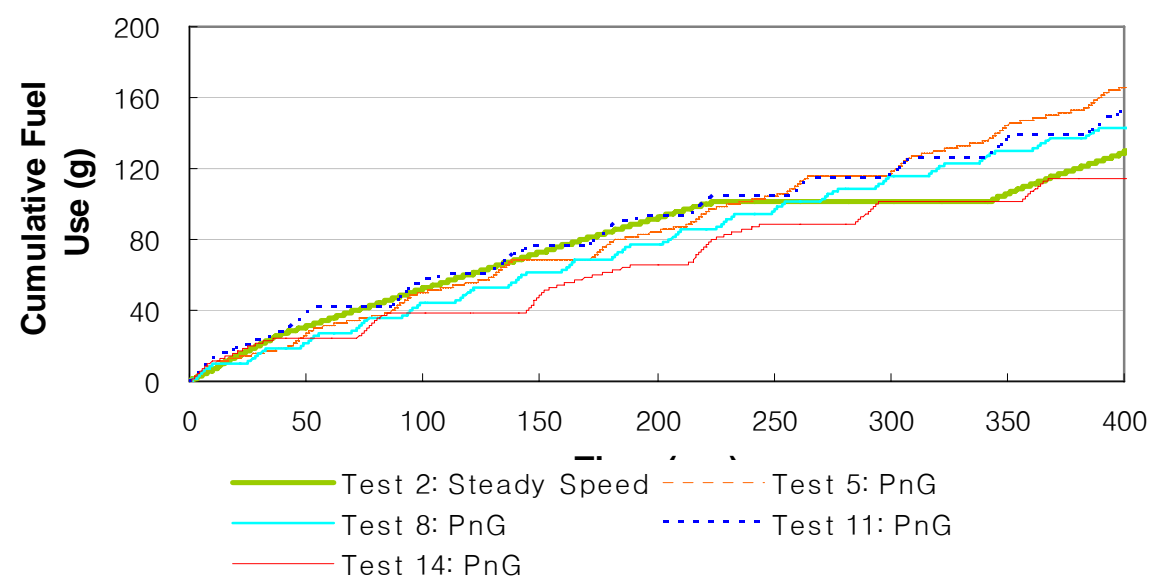

Figure 6.10 Cumulative fuel use comparison for PnG in $30-40 \mathrm{mph}$ and steady speed at 35 mph, 2004 Toyota Prius

\section{Cumulative Fuel Use for PnG in 40 - $50 \mathrm{mph}$ and Steady} Speed at 45 mph, 2004 Toyota Prius

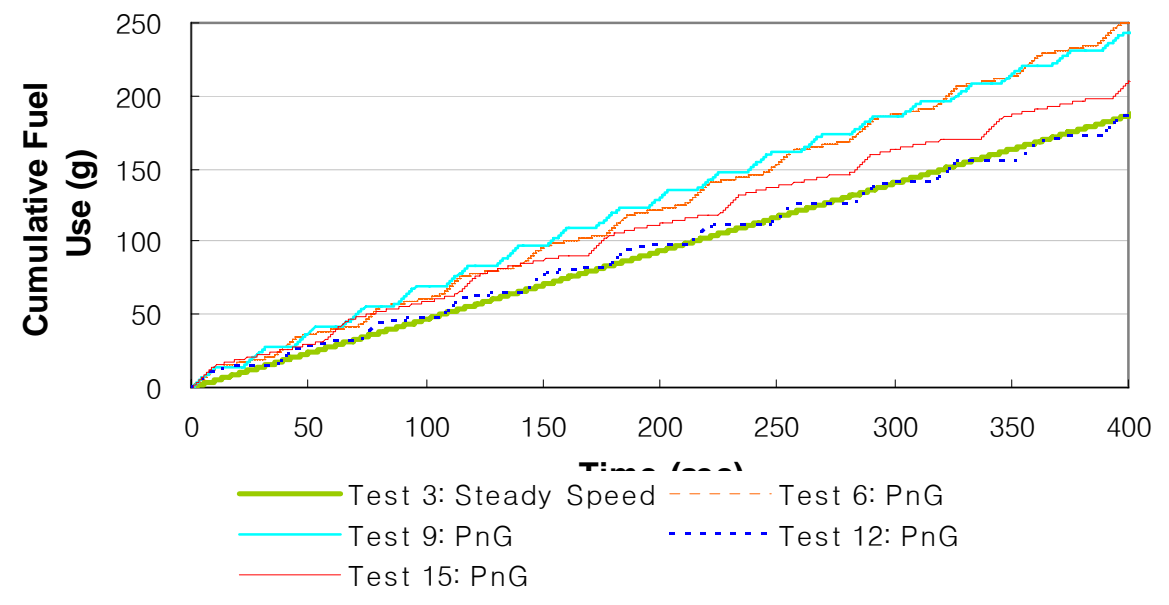

Figure 6.11 Cumulative fuel use comparison for PnG in $40-50 \mathrm{mph}$ and steady speed at 45 mph, 2004 Toyota Prius

\subsection{Fuel Economy Results}

The fuel economy for steady speed and PnG cases is calculated based on the cumulative fuel use and the distance from vehicle testing. In order to obtain accurate results, one of the SOC correction methods introduced in section 2.5 is used. Also the fuel economy results are 
compared to the simulation results here. The following sections present SOC correction for fuel economy of the $\mathrm{HEV}$, fuel economy results and comparison with simulation results one by one.

\subsubsection{SOC Correction}

A few SOC correction methods are introduced early in section 2.5. In order to get more accurate fuel economy data of the PnG cases, one of SOC correction methods is used because it is very difficult to get a swing of SOC over the PnG drive cycles. Thus, each PnG cycle of one case is extracted first to estimate fuel consumption as shown in Figure 6.12 below. After that the fuel consumption in $\mathrm{L} / 100 \mathrm{~km}$ for each cycle is plotted over the net electric energy consumption in $\mathrm{Wh} / \mathrm{mile}$. The SOC data from vehicle testing gives only $0.5 \%$ increment and it can cause an error when fuel economy is adjusted. Hence, more accurate net electric energy consumption is used instead of delta SOC for SOC correction. From the plotted data, a linear regression is used to obtain SOC corrected fuel consumption and they are converted to fuel economy in miles per gallon (mpg). In Figure 6.12, the y-intercept of the linear regression line at zero net electric energy consumption, $3.896 \mathrm{~L} / 100 \mathrm{~km}$, represents the SOC corrected fuel consumption. More details about the linear regression are explained in Appendix C. 


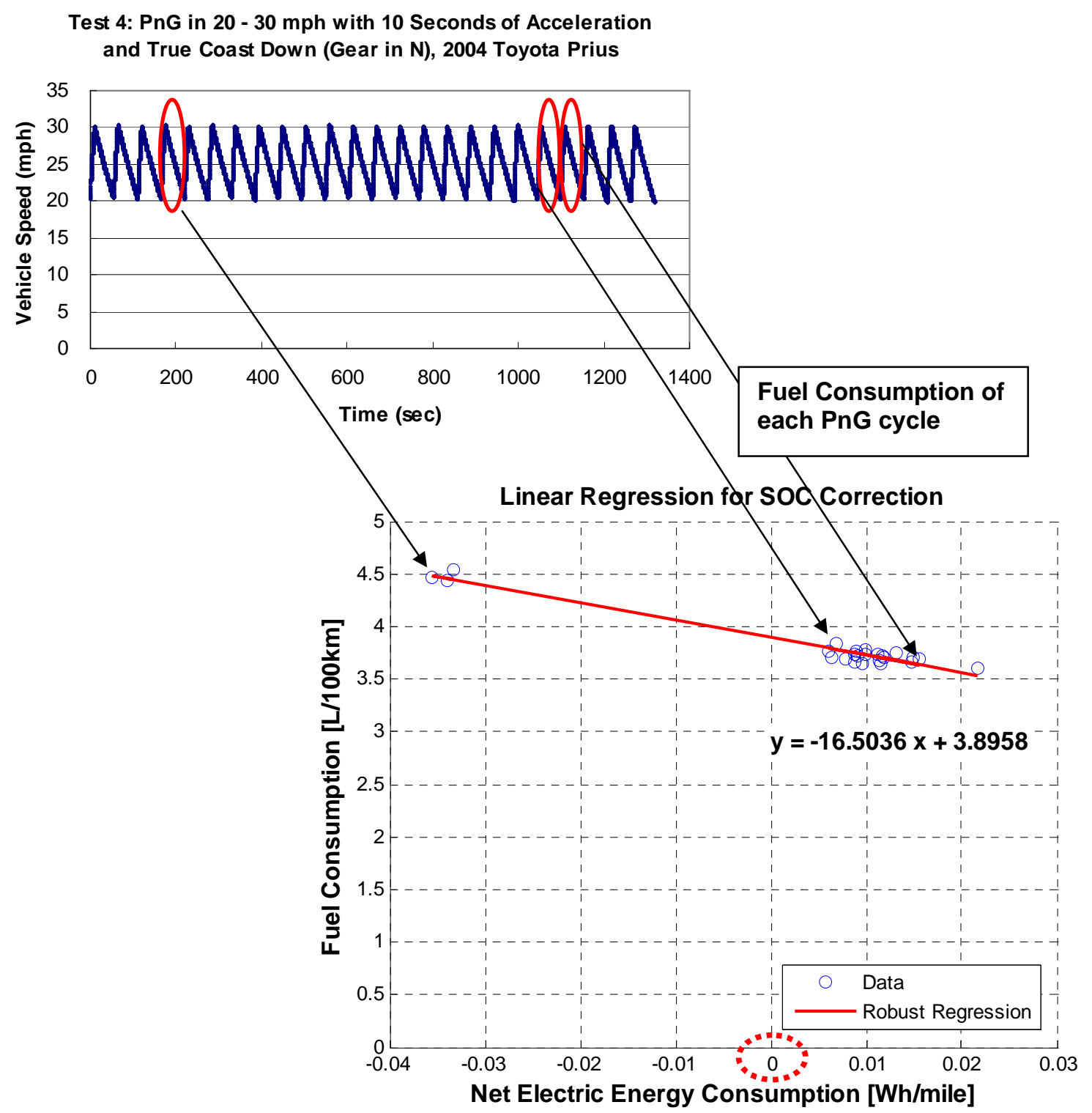

Figure 6.12 Example of SOC correction for fuel economy of PnG (test 4), 2004 Toyota Prius

The fuel economy of the steady speed cases for the HEV is obtained with the same method as illustrated in Figure 4.3 because the data from vehicle testing show a swing of SOC over the steady speed drive cycles. It is also more convenient to extract the data in the interval with the same initial and final SOCs for SOC corrected fuel economy. 


\subsubsection{Fuel Economy}

The SOC corrected fuel economy for the PnG and the steady speed cases from vehicle testing is obtained. As previously mentioned, two different approaches for SOC correction are used here. The fuel economy results of the HEV from vehicle testing are summarized in Table 6.1 and they are plotted in Figures 6.10, 6.11 and 6.12 below. More detailed results from vehicle testing are tabulated in Tables B.11, B.12 and B.13 in Appendix B.

Table 6.1 Fuel economy results for the HEV from vehicle testing, 2004 Toyota Prius

\begin{tabular}{|c|c|c|c|c|c|c|}
\hline & & Steady Speed & \multicolumn{4}{|c|}{ PnG } \\
\hline $\begin{array}{c}20-30 \\
\text { mph }\end{array}$ & Test Case & Test 1 & Test 4 & Test 7 & Test 10 & Test 13 \\
\cline { 2 - 7 } & Fuel Economy $(\mathrm{mpg})$ & $117.2($ at $25 \mathrm{mph})$ & 60.4 & 103.1 & 118.5 & 123.5 \\
\hline \multirow{2}{*}{$\begin{array}{c}30-40 \\
\mathrm{mph}\end{array}$} & Test Case & Test 2 & Test 5 & Test 8 & Test 11 & Test 14 \\
\cline { 2 - 7 } & Fuel Economy $(\mathrm{mpg})$ & $92.3($ at $35 \mathrm{mph})$ & 61.6 & 91.3 & 102.4 & 101.0 \\
\hline \hline $\begin{array}{c}40-50 \\
\text { mph }\end{array}$ & Test Case & Test 3 & Test 6 & Test 9 & Test 12 & Test 15 \\
\cline { 2 - 7 } & Fuel Economy $(\mathrm{mpg})$ & $75.3($ at $45 \mathrm{mph})$ & 57.5 & 73.2 & 74.2 & 75.6 \\
\hline
\end{tabular}

The fuel economy of the steady speed driving (test 1) at $25 \mathrm{mph}$ is $117.2 \mathrm{mpg}$ as seen in Figure 6.13. However, the PnG case (test 4) with true coast down shows much lower fuel economy, $60.4 \mathrm{mpg}$ (48\% lower) than that of the steady speed case. Again, the engine still consumes fuel during coasting as stated in section 6.1. The case of engine compression braking emulated by the HEV (test 7) finally shows $12 \%$ lower fuel economy than the steady speed driving because the VMT during coasting is relatively shorter than the other PnG cases even if the engine is off and the battery is charged by the regenerative system. It is actually expected from the conventional vehicle cases but it is verified here again. The PnG case with the same vehicle speed profile as the test 4 and gear in D (test 7) shows a little higher fuel economy and the PnG case with slower deceleration rates (test 13) gives $5.4 \%$ better fuel economy compared to that of the test 1 . In short, the fuel economy by the PnG driving strategy in $20-30 \mathrm{mph}$ is improved in very small scale because the HEV system does engine load leveling in contrast to PSAT simulation. Thus, the engine runs to charge the battery in high efficiency region for a while until the SOC is high enough and the EV mode makes the engine off to avoid engine operation in low efficiency region in the vehicle testing. This is the reason why the fuel economy of the steady speed is improved significantly in the vehicle testing compared to the simulation. In short, the engine 
load leveling with battery energy storage by the HEV control strategy improves fuel economy significantly, as the PnG driving strategy does by storing KE in real vehicle testing.

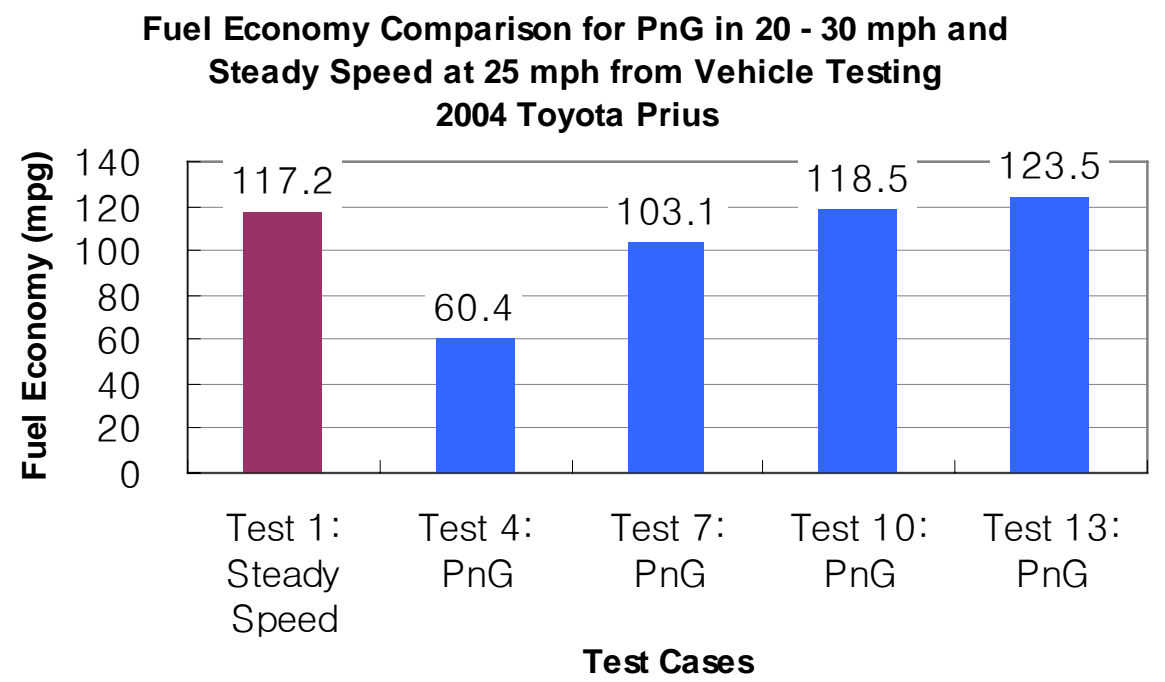

Figure 6.13 SOC corrected fuel economy results for PnG in $20-30 \mathrm{mph}$ and steady speed at 25 mph from vehicle testing, 2004 Toyota Prius

In $30-40$ mph of the PnG driving, the fuel economy improvement is a little better than in $20-$ $30 \mathrm{mph}$. As shown in Figure 6.14, the fuel economy of the PnG case with the same vehicle speed profile (test 11) as the test 5 and gear in D has the largest improvement, approximately $11 \%$, compared to the steady speed case (test 2 ). The PnG case with slower deceleration rates (test 14) also shows about $9.5 \%$ of fuel economy improvement. However, the PnG case with true coast down (test 5) and the PnG case with emulated engine compression braking (test 8) give lower fuel economy than the test 2 . Interestingly, the test 8 gets very close to the fuel economy of the test 2 in $30-40 \mathrm{mph}$ speed range. It seems that the regenerative brake system gives more benefit for this case compared to the low speed range case.

In the higher speed range, $40-50 \mathrm{mph}$, the fuel economy improvement by the PnG driving is almost zero as plotted in Figure 6.15 below. The PnG cases except the test 6 shows a little better or worse fuel economy (within $\pm 2 \mathrm{mpg}$ ) than the steady speed case. The test 12 has the same vehicle speed profile as the test 3 and the engine is mostly off during coasting as shown in Figure $6.7-$ (c). However, the fuel rate of the test 12 is much higher $(\sim 1.2 \mathrm{~g} / \mathrm{s})$ than that of the test 2 $(\sim 0.45 \mathrm{~g} / \mathrm{s})$ even if they both have the same distance. This higher rate can be easily seen from the 
cumulative fuel use plot in Figure 6.11 - (c) (see the dashed lines). In the test 14, the VMT of each cycle is longer than the other PnG cases but the engine still consumes fuel to follow the vehicle speed (powered deceleration) between 40 and $50 \mathrm{mph}$. Again, the test 6 shows the lowest fuel economy because of more fuel consumption during coasting.

\section{Fuel Economy Comparison for PnG in $\mathbf{3 0}$ - 40 mph and Steady Speed at $35 \mathrm{mph}$ from Vehicle Testing 2004 Toyota Prius}

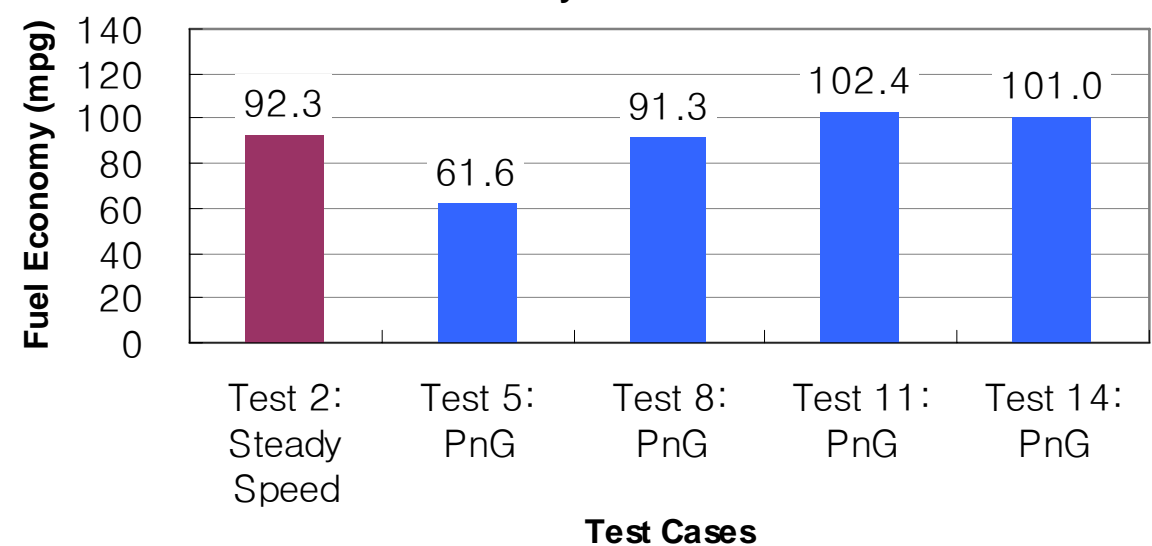

Figure 6.14 SOC corrected fuel economy results for PnG in $30-40 \mathrm{mph}$ and steady speed at 35 mph from vehicle testing, 2004 Toyota Prius

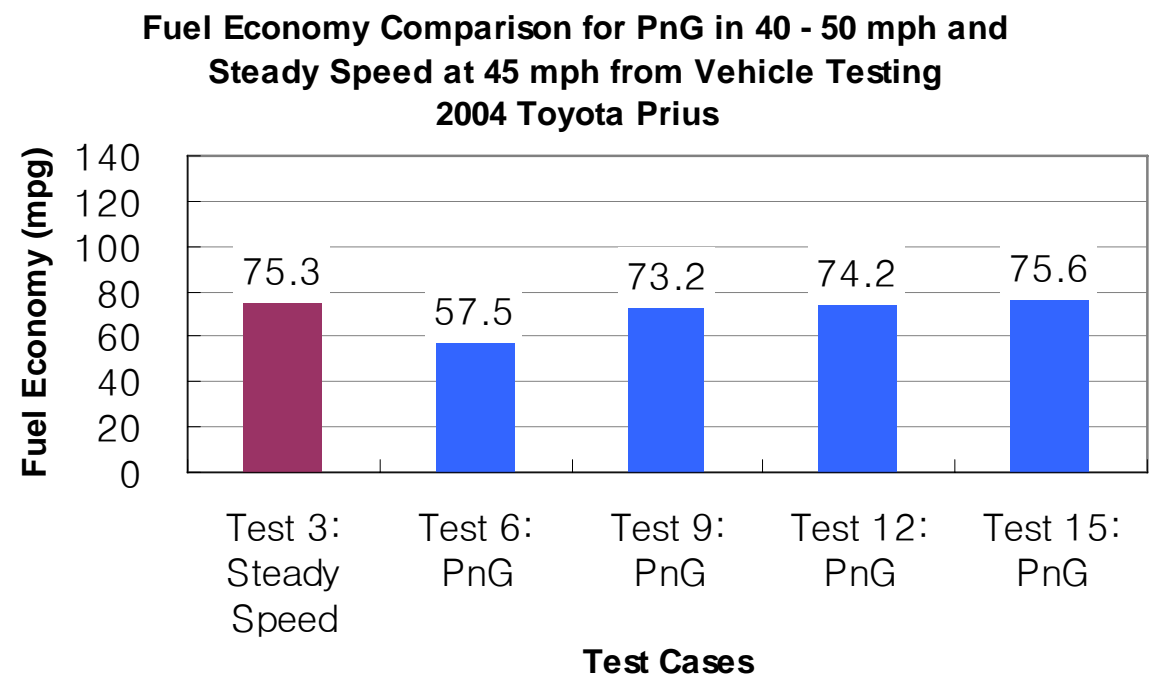

Figure 6.15 SOC corrected fuel economy results for PnG in $40-50 \mathrm{mph}$ and steady speed at 45 mph from vehicle testing, 2004 Toyota Prius 
In summary, the fuel economy results for the HEV by the PnG driving strategy from vehicle testing are discussed above. The results shows that the fuel economy improvement of the PnG cases over the steady speed cases is not very large compared to the simulation results in Chapter 4. Especially, the high speed PnG cases do not improve the fuel economy by engine on/off strategy by PnG over the steady speed case because the road load power is high enough to get good engine efficiency. The next section will compare the results from vehicle testing to the simulation results from PSAT in details.

\subsubsection{Comparison with Simulation Results}

The fuel economy results from vehicle testing show that the impact of the PnG driving on fuel economy is not very significant compared to the simulation results from PSAT. Figure 6.16 below shows the comparison between the simulation and vehicle testing results in terms of fuel economy. The compared PnG simulation results are only for 10 seconds of acceleration because only that acceleration time case is tested. Note that the fuel economy results shown in Figure 6.16 are SOC corrected data.

Overall, the fuel economy of the steady speed cases is higher from vehicle testing than that from PSAT simulation. In contrast to that, the fuel economy of the PnG cases is lower from vehicle testing than that from PSAT simulation. Thus, the fuel economy improvement by the PnG driving strategy in vehicle testing is much smaller. The steady speed cases show much larger difference (43\% higher in vehicle testing) than the PnG cases, specifically the $25 \mathrm{mph}$ case. The constant fuel consumption in the engine (see Figure 4.11) over the steady speed drive cycle is the reason why the simulation result has lower fuel economy than the vehicle testing results at 25 mph. In Figure 6.1 - (a), the HEV turns the engine on and off depending on the level of SOC in vehicle testing which means that it uses mostly motor at low speed and turns engine on to propel the vehicle and charge the battery when the SOC is low. However, the HEV model in PSAT simulation does not behave like that and the SOC is maintained around $68 \%$ because the engine mostly propels the vehicle. From this analysis, it can be assumed that the current HEV model in PSAT has some missing control strategy for particular user defined drive cycles. The difference of fuel economy for the steady speed cases becomes smaller as the speed is increased. 
Fuel Economy Comparison between Simulation and Vehicle Testing, 2004 Toyota Prius

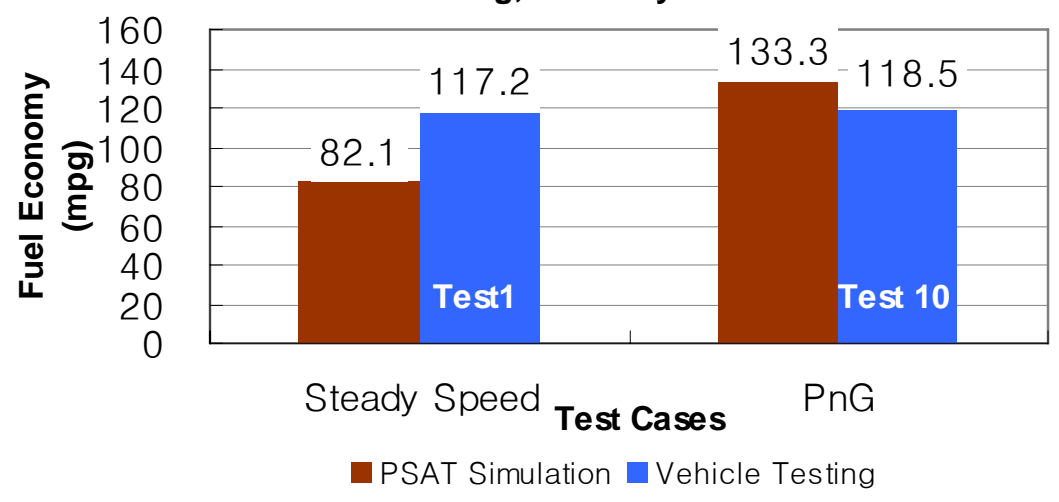

(a) PnG in $20-30 \mathrm{mph}$ and steady speed at $25 \mathrm{mph}$

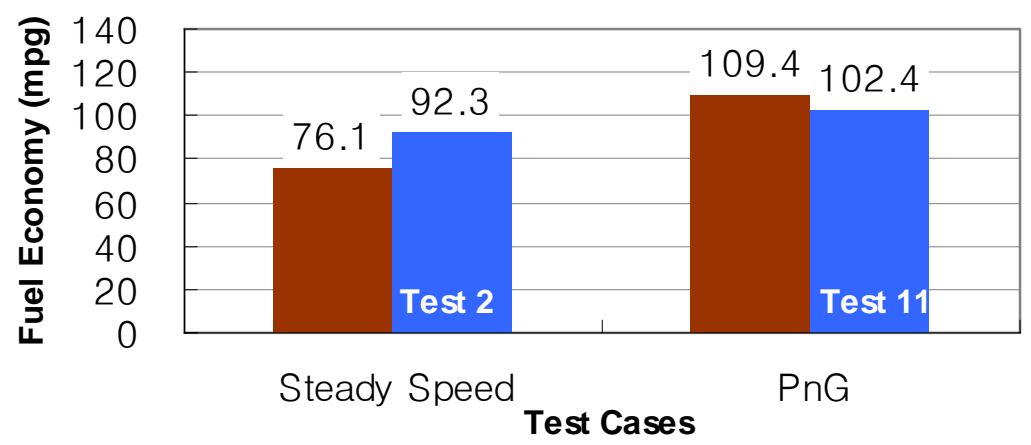

PSAT Simulation $\square$ Vehicle Testing

(b) PnG in $30-40 \mathrm{mph}$ and steady speed at $35 \mathrm{mph}$

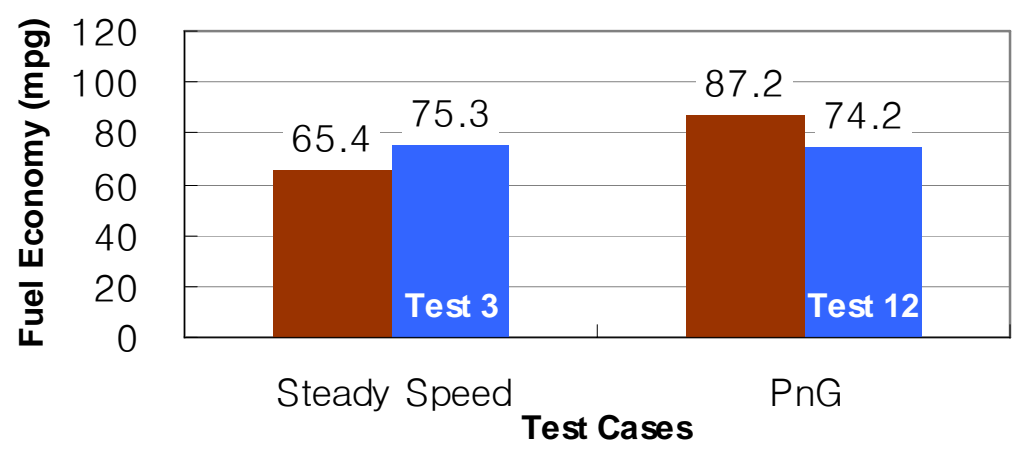

PSAT Simulation $\square$ Vehicle Testing

(c) PnG in $40-50 \mathrm{mph}$ and steady speed at $45 \mathrm{mph}$

Figure 6.16 Fuel economy comparison between simulation and vehicle testing results for PnG with 10 seconds of acceleration and steady speed cases, 2004 Toyota Prius 
For the PnG cases, the difference of fuel economy between simulation and vehicle testing is much smaller than that of the steady speed cases. The $30-40 \mathrm{mph}$ cases show smallest difference (6.8 \%) in Figure 6.16 - (b). If the fuel rates of the PnG cases in $20-30 \mathrm{mph}$ are compared each other in Figure 4. 11 and Figure 6.3 - (a), the vehicle testing shows a little higher fuel rate than the simulation and it can be one of the reasons why the fuel economy from vehicle testing is lower than that from simulation. In addition, the PnG tests (tests 10, 11 and 12) described above are not for true coast down conditions with gear in $\mathrm{N}$ during coasting. The driver should push the accelerator pedal to follow the same vehicle speed profile as the PnG case with true coast down during deceleration and the HEV consumes additional energy which is either fuel energy or electric energy. It obviously increases fuel consumption because more energy is consumed compared to the simulation cases. Figure 6.17 below clearly shows that with the ratio of fuel use during acceleration and coasting over the total fuel use for the PnG cases. Thus, the HEV consumes approximately $14-18 \%$ more fuel in vehicle testing due to the condition stated above. Note that the HEV turns on the engine during coasting if the gear is in $\mathrm{N}$ position and it results in much worse fuel economy as discussed earlier with Figures $6.10,6.11$ and 6.12 so those cases are not compared here.

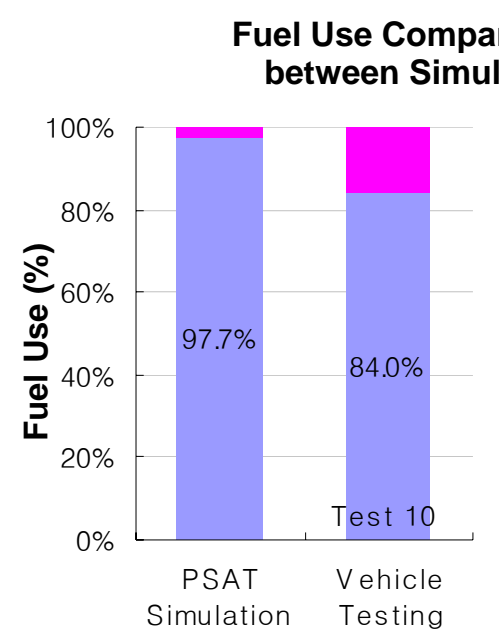

PnG: 20 - $30 \mathrm{mph}$

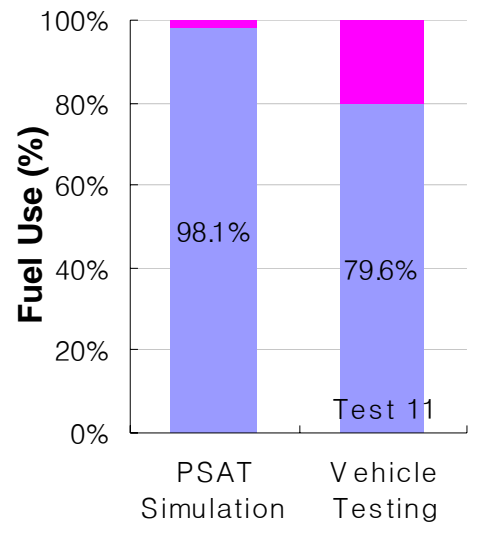

PnG: 30 - $40 \mathrm{mph}$

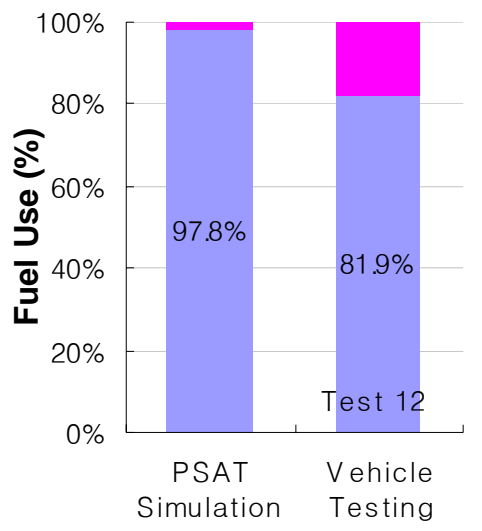

PnG: 40 - 50 mph

Fuel Use during Coasting

Fuel Use during Acceleration

Figure 6.17 Fuel use comparison of $\mathrm{PnG}$ during acceleration and coasting between simulation and vehicle testing (10 seconds of acceleration), 2004 Toyota Prius 
Consequently, the fuel economy improvement by the PnG driving strategies in vehicle testing is much smaller for the $20-30 \mathrm{mph}$ and $30-40 \mathrm{mph}$ speed ranges or even a little worse for the 40 $-50 \mathrm{mph}$ speed range than the simulation results. However, the fuel economy can be sometimes improved in the HEV by the modified PnG driving strategies, especially at low speed ranges; 20 $-30 \mathrm{mph}$ and $30-40 \mathrm{mph}$.

\subsection{Efficiency of Kinetic Energy Storage}

The overall efficiency of KE storage from vehicle testing for the HEV is estimated over the drive cycles using equation (3.33) again and plotted in Figure 6.18. The green, blue and pink bars represent the efficiency of KE storage for $20-30 \mathrm{mph}, 30-40 \mathrm{mph}$ and $40-50 \mathrm{mph}$ cases respectively.

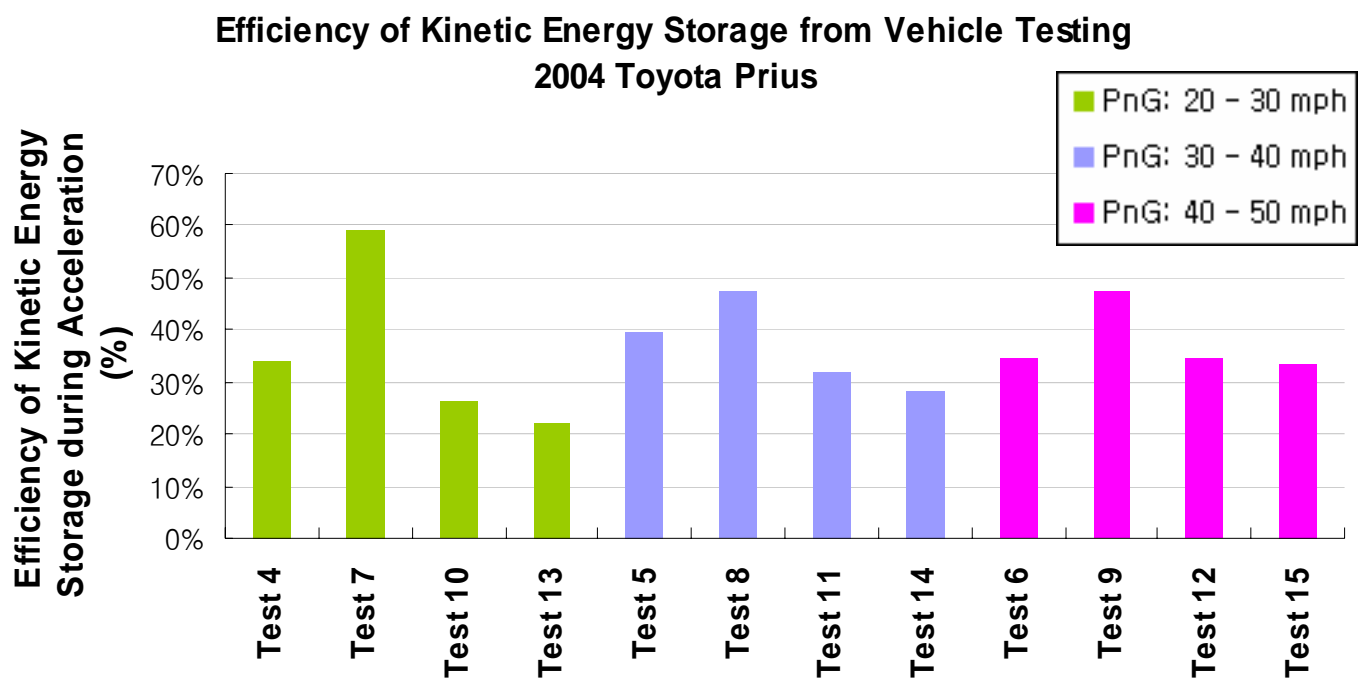

Figure 6.18 Efficiency of KE storage during acceleration for PnG (10 seconds of acceleration), 2004 Toyota Prius

Most of the efficiency shows approximately $30 \%$ except a few cases. First of all, the emulated engine compression braking cases (tests 7,8 and 9) show much higher efficiency of KE storage for all three different speed ranges. In those cases, engine compression braking makes the VMT shorter due to frictional losses so they have more frequent acceleration phases and more inertial energy required over the drive cycle than the other PnG cases. In contrast to that, the SOC is 
increased by regenerative braking during engine compression braking, so the HEV uses battery power for acceleration in the low speed ranges $(20-30 \mathrm{mph}$ and $30-40 \mathrm{mph})$ in Figure 6.7. It results in less fuel consumption than expected in the PnG driving and much higher efficiency of KE. Note that the efficiency of KE storage is the inertial energy during acceleration divided by the fuel energy difference between the steady speed and PnG cases during acceleration. However, the fuel economy improvement is not achieved by those PnG driving cases with engine compression braking.

On the contrary, the largest fuel economy improvement is achieved when the efficiency of KE storage is lower than the PnG cases with engine compression braking; test 13 for $20-30 \mathrm{mph}$ speed range and test 11 for $30-40 \mathrm{mph}$ speed range. In those cases, the engine mostly produces power to accelerate the vehicle without EV mode and the battery is charged as well during acceleration by the engine power. Thus, the fuel energy difference between the PnG and steady speed driving becomes larger than the engine compression braking cases and it results in lower efficiency of KE storage. As discussed so far, the efficiency of KE storage cannot be used directly to estimate the benefit of the PnG driving strategy on fuel economy in an HEV due to engine operation and fuel use in the coast phase.

\subsection{Summary}

The vehicle testing for the HEV using different PnG and driving strategies is performed with steady speed driving. According to the results, the HEV system does not behave in the same way in simulation as vehicle testing. In steady speed driving condition, the HEV uses electric power very often at low speed. However, the simulation results show that the engine is operating mostly and consumes fuel. In higher steady speed, both results show that the HEV uses mostly engine power to maintain the vehicle speed at $45 \mathrm{mph}$. In PnG driving cases, the HEV shows unexpected behavior that the engine consumes fuel even if the gear is $\mathrm{N}$ during coasting. It results in much worse fuel economy than the steady speed cases. For the PnG cases with emulated engine compression braking, the fuel economy is a little lower than the steady speed cases. In contrast to that, the PnG cases with the same vehicle speed profile and gear in D show that the fuel economy is improved by $1-11 \%$ except the $40-50 \mathrm{mph}$ of PnG case. In the PnG 
cases with slower deceleration rates in $20-30 \mathrm{mph}$ and $30-40 \mathrm{mph}, 5 \%$ and $9.5 \%$ improvement of fuel economy is achieved compared to that of steady speed cases. However, the fuel economy improvement in $40-50 \mathrm{mph}$ by the same PnG strategy is very small. In short, the fuel economy benefit by the PnG driving strategy becomes smaller at higher speed because the road load power itself (both aerodynamic drag resistance and rolling resistance) is enough to raise engine efficiency to a high level so there is no need to add extra power from acceleration or battery charging.

In comparison with the simulation results, the fuel economy improvement by the PnG driving is relatively smaller in vehicle testing. There are a few possible reasons for that but the main reason is that the HEV uses electric power more often without any fuel consumption in steady speed from vehicle testing compared to PSAT simulation. In other words, the real test vehicle does more engine load leveling compared to the control strategy in PSAT which is tuned for dynamic standard drive cycles like UDDS. However, it is shown that the PnG driving strategy can possibly improve fuel economy up to approximately $9.5 \%$ for the HEV with a modified PnG driving strategy (powered deceleration) over the steady speed driving at low speed. 


\section{Chapter 7 Conclusions and Future Work}

One of the eco driving strategies, the pulse and glide $(\mathrm{PnG})$ method is simulated and analyzed for a conventional vehicle and an HEV in this research. In order to investigate the impact of the PnG driving strategy on fuel consumption compared to the steady speed driving, various PnG drive cycles are generated for different parameters such as speed range, acceleration time, speed difference and deceleration time. The generated $\mathrm{PnG}$ drive cycles are simulated for a conventional vehicle by a simple vehicle model developed in the beginning of this study. In addition, in-depth simulation is performed for a conventional and a hybrid electric vehicle in PSAT. In the simulation results, the PnG driving shows a large potential for improving fuel economy for both conventional and hybrid electric vehicles. An HEV is tested on a dynamometer at ANL to verify the benefit of fuel economy by the PnG driving strategy. Finally, this study leads to the following conclusions.

1. At first, the results by the simple vehicle model for a conventional vehicle show that the fuel consumption improvement by the PnG driving strategy is larger at low speed range and for shorter acceleration time with a fixed gear condition for each speed range. If acceleration is shorter, the engine load is increased but the engine efficiency is higher and the engine operating time is short. This load increase results in the largest fuel economy improvement by the PnG driving strategy compared to steady speed driving. However, in the higher speed range, the benefit of fuel economy by the PnG driving is smaller due to much higher aero drag resistance which is proportional to the square of vehicle speed, higher road load power, and thus higher engine load for steady speed driving. 
2. The larger speed difference $(20 \mathrm{mph})$ case results in fuel economy similar to the $10 \mathrm{mph}$ speed difference case. However, since the large speed difference case does not show significant improvement of fuel economy and might need gear shifting, this case was excluded for further simulation in this research. In addition, the drivability and ride quality which are not considered in this study can be an issue at such a large speed difference (20 mph).

3. The deceleration time was described by a simple engine compression braking model for the conventional vehicle. The results show that the engine absorbs wheel torque through the engaged clutch (engine frictional loss) even if the engine is off and it makes the VMT shorter in that condition. In other words, the engine braking is still frictional loss. Therefore, the fuel economy becomes worse even if the amount of cumulative fuel use during acceleration is the same as the PnG case with disengaged gear.

4. After the simulation using the simple vehicle model, in-depth simulation for a conventional vehicle is first performed in PSAT. According to the results, the fuel economy is improved by the PnG driving strategy compared to the steady speed driving even if the improvement is somewhat lower than that of the simple vehicle model due to simplified parameters. The fuel consumption is largely reduced in the lower speed range and the shorter acceleration time compared to the steady speed cases. The largest fuel economy improvement occurs at 5 seconds of acceleration in $20-30 \mathrm{mph}$ speed range for the conventional vehicle. However, the PnG cases with 5 seconds of acceleration for higher speed ranges are not achieved due to engine torque limit.

5. A power-split HEV is also simulated in PSAT for the same PnG and steady speed cases as the conventional vehicle. The fuel economy improvement is a little smaller than the conventional vehicle results because the HEV is already well optimized for reducing fuel consumption and using electric energy by preventing the engine from operating in a low efficiency region. In other words, the HEV has battery energy storage and it does engine load leveling. However, it still shows some potential of the PnG driving strategy. Also the trend of the fuel economy improvement is very similar to the conventional vehicle except the PnG cases with 5 seconds of acceleration. In those cases, the fuel economy drops significantly compared to the 10 seconds of acceleration. The fuel economy of the $\mathrm{PnG}$ in $40-50 \mathrm{mph}$ speed range with 5 
seconds of acceleration is even very close ( $4 \%$ of improvement) to the steady speed case. This behavior occurs because the HEV cannot produce enough power to propel the vehicle by the engine only in higher acceleration ( 5 second cases) so the motor assists the engine with electric energy from the battery but the engine then consumes fuel again during coasting if the SOC of the battery is low. Consequently, it results in more fuel consumption in the HEV than the PnG cases with 10 seconds of acceleration. In other words, there is no engine efficiency benefit once the engine load is high enough.

6. From the in-depth simulation by PSAT, vehicle testing for the HEV was conducted to verify the impact of the PnG driving strategy on fuel consumption in a real vehicle. In vehicle testing, different deceleration rates including emulated engine compression braking are more focused after further discussion because the HEV has regenerative braking capability and it can possibly affect fuel consumption by the PnG driving strategy. Also, only 10 seconds of acceleration cases which show the largest fuel economy improvement are tested due to the limited test schedule at ANL. According to the test results, the fuel economy of the steady speed driving cases is largely improved compared to the simulation results because the engine is on and off depending on the SOC and saves fuel in real vehicle testing. However, the engine is always on in PSAT simulation because some of the details of HEV control strategy are not well demonstrated for the PnG drive cycles. In the PnG cases, the fuel economy by the PnG driving with true coast down is much worse than the steady speed cases because the engine is always idling during coasting. The PnG cases with emulated engine compression braking also show worse fuel economy than the steady speed cases. However, the PnG cases with powered deceleration improve fuel economy up to $9.5 \%$ and $11 \%$ in $20-30 \mathrm{mph}$ and $30-40 \mathrm{mph}$ respectively because there are round trip losses between the engine/motor and the battery in steady speed but the PnG driving strategy can reduce the losses. In vehicle testing for the HEV, the fuel economy improvement is achieved in low speed ranges; $20-30 \mathrm{mph}$ and $30-40 \mathrm{mph}$, but there is no benefit of the PnG driving strategy in $40-50 \mathrm{mph}$.

7. The newly introduced term, the efficiency of KE storage, is calculated for both conventional and hybrid electric vehicles. This term represents whether storing KE in vehicle inertia with additional fuel consumption during acceleration is more efficient or not compared to the steady speed driving. Specifically, the efficiency of KE can be compared to the efficiency of engine 
load leveling with battery energy storage for an HEV. The results describe that the efficiency of $\mathrm{KE}$ storage is higher than the overall engine efficiency during acceleration over the drive cycles in simulation. Unfortunately, it cannot be compared with the engine efficiency from vehicle testing due to inaccuracy of test data, for example, the overestimated engine output torque. In the HEV analysis, it is found that the higher efficiency of KE cannot directly estimate fuel economy improvement by the PnG driving strategy when the engine operates in the coast phase so engine load leveling with battery energy storage should be considered together.

In this study, the simulation results show large potential (up to $92 \%$ for the conventional vehicle and up to $62 \%$ for the HEV in 20 - $30 \mathrm{mph}$ range) of the PnG driving strategy on fuel economy for both conventional and hybrid electric vehicles. In the conventional vehicle, the benefit of the PnG driving strategy is larger because there is no energy storage like a battery in an HEV so storing $\mathrm{KE}$ in vehicle inertia can improve fuel economy. However, it is proved that the fuel economy improvement of the HEV is much smaller than that of the conventional vehicle through real vehicle testing at ANL. In addition, the HEV behaves differently for the PnG and steady speed drive cycles in the PSAT model because some of the details of HEV control strategy are not well demonstrated. Thus, the PSAT model estimates higher fuel economy improvement by the PnG driving strategy in simulation. In conclusion, engine load leveling with the battery by the HEV control strategy in steady speed driving is as efficient as storing KE in vehicle inertia by the PnG driving strategy but the fuel economy improvement $(1-11 \%)$ is achieved in $20-30$ mph and 30-40 mph speed ranges by the PnG driving strategy even if it is much smaller than the simulation results. Also, the PSAT simulation can misstate the fuel economy results for both the steady speed and PnG drive cycles, especially for the HEV.

After completing this research, a few more interesting ideas can be studied in the future. First of all, the PnG cases with only 10 seconds of acceleration are tested with the HEV due to limited time. However, if more PnG cases with different acceleration times are tested, the results might be different from the simulation results and show additional reduction in fuel consumption. Also, the conventional vehicle with a manual transmission is not tested for the PnG driving strategy on a dynamometer unfortunately, but it could be interesting to compare with the simulation results. The last future work could be applying the PnG driving for a parallel or a series HEV rather than 
the split HEV tested in this research because they have different powertrain configurations and control strategies, and it might affect the results as well. 


\section{References}

[1] U.S. Finished motor gasoline product supplied data, Energy Information Administration (EIA), http://tonto.eia.doe.gov/dnav/pet/hist/mgfupus1a.htm

[2] SAE Supermileage, http://students.sae.org/competitions/supermileage/

[3] Shell Eco Marathon, http://www.shell.com/eco-marathon/

[4] Golden Rules of Eco-Driving, http://www.ecodrive.org

[5] Eco-Driving: Ten Easy Tips for Saving Fuel, http://www.ford.com/innovation/environmentally-friendly/eco-driving

[6] THE 2010 FORD FUSION HYBRID TOPS 1,445.7 MILES ON ONE TANK OF GAS http://ford.digitalsnippets.com/2009/04/28/the-2010-ford-fusion-hybrid-tops-14457-mileson-one-tank-of-gas/

[7] K. Reynolds, "In Search of High Mileage - America's mpg champions meet their match," Road and Track, http://metrompg.com/posts/xfi-pulse-and-glide.htm

[8] "Prius Marathoners Top 100 mpg - Testing the Limits of Fuel Economy," Toyota Hybrid Synergy View Letter, Fall 2005, http://www.toyota.com/html/hybridsynergyview/2005/fall/marathon.html

[9] B. Jawad, et al., "Best Practices for An SAE Supermileage Vehicle," SAE Paper 2001-012469,2001

[10] G. Lechner and H. Naunheimer, “Automotive Transmissions,” Springer, 1999 
[11] 2009 F1 Technical Regulations, http://www.fia.com/resources/documents/1151088479_2009_F1_TECHNICAL_REGULA TIONS.pdf

[12] Keith Collantine, "F1 2010 Rules: KERS to stay," 19 August, 2009, http://www.f1 fanatic.co.uk/2009/08/19/f1-2010-rules-kers-to-stay/

[13] Doug Cross, "Optimization of Hybrid Kinetic Energy Recovery Systems (KERS) for Different Racing Circuits,” SAE Paper 2008-01-2956, 2008

[14] P. Sharer, et al., "Impact of Drive Cycle Aggressiveness and Speed on HEVs Fuel Consumption Sensitivity," SAE Paper 2007-01-0281, 2007

[15] Michael Duoba, Henning Lohse-Busch and Theodore Bohn, "Investigating Vehicle Fuel Economy Robustness of Conventional and Hybrid Electric Vehicles," Proceedings of the EVS-21, The $21^{\text {st }}$ Worldwide Battery, Hybrid and Fuel Cell Electric Vehicle Symposium and Exposition, 2005

[16] F. An and D. Santini, "Mass Impacts on Fuel Economies of Conventional vs. Hybrid Electric Vehicles," SAE Paper 2004-01-0572, 2008

[17] C Reynolds and N Kandlikar, "How hybrid-electric vehicles are different from conventional vehicles: the effect of weight and power on fuel consumption," Environmental Research Letters, Vol. 2, No. 2, 2007

[18] M. Duoba, et al., "Analysis of Power-Split HEV Control Strategies Using Data from Several Vehicles," SAE Paper 2007-01-0291, 2007

[19] Volkan Sezer, et al., "Maximizing Overall Efficiency Strategy (MOES) for Power Split Control of a Parallel Hybrid Electric Vehicle," SAE Paper 2008-01-2682, 2008

[20] Jinming Liu and Huei Peng, "Control Optimization for a Power-Split Hybrid Vehicle," Proceedings of the 2006 American Control Conference, 2006

[21] Joonyoung Park, Younkug Park and Jahng-Hyon Park, "Real-Time Powertrain Control Strategy for Series-Parallel Hybrid Electric Vehicles,” SAE 2007-01-3472, 2007

[22] S. Boyd and D. Nelson, "Hybrid Electric Vehicle Control Strategy Based on Power Loss Calculations," SAE Paper 2008-01-0084, 2008

[23] S. Boyd, "Hybrid Electric Vehicle Control Strategy Based on Power Loss Calculations," Master's Thesis at Virginia Tech, August 2006 
[24] K. Johnson, "A Plug-in Hybrid Electric Vehicle Loss Model to Compare Well-to-Wheel Energy Use from Multiple Sources,” Master's Thesis at Virginia Tech, July 2008

[25] M. Baglione, M. Duty and G. Pannone, "Vehicle System Energy Analysis Methodology and Tool for Determining Vehicle Subsystem Energy Supply and Demand," SAE Paper 2007-010398, 2007

[26] Soon-il Jeon, et al., "Multi-Mode Driving Control of a Parallel Hybrid Electric Vehicle Using Driving Pattern Recognition," ASME Journal of Dynamic Systems, Measurement, and Control, Vol. 124, pp.141 - 149, 2002

[27] Chan-Chiao Lin, et al., "Driving Pattern Recognition for Control of Hybrid Electric Trucks," International Journal of Vehicle Mechanics and Mobility: Vehicle System Dynamics, Vol. 42, pp. $41-57,2004$

[28] Nobuki Kawamoto, et. al., "Development of New 1.8-Liter Engine for Hybrid Vehicles," SAE 2009-01-1061, 2009

[29] Edward Bass and Timothy Alfermann, "The Influence of Idle, Drive Cycle and Accessories on the Fuel Economy of Urban Hybrid Electric Buses - Chassis Dynamometer Test," SAE 2003-01-3438, 2003

[30] John Bishop, et al., "An Engine Start/Stop System for Improved Fuel Economy," SAE 2007-01-1777, 2007

[31] Moritaka Matsuura, Koji Korematsu and Junya Tanaka, "Fuel Consumption Improvement of Vehicles by Idling Stop," SAE 2004-01-1896, 2004

[32] Office of Transportation and Air Quality, "Compilation of State, County, and Local AntiIdling Regulations,” EPA 420-B-06-004, April, 2006

[33] K. Muta, et al., "Development of New-Generation Hybrid System THS II - Drastic Improvement of Power Performance and Fuel Economy," SAE Paper 2004-01-0064, 2004

[34] Takuji Matsubara, et al., "Development of New Hybrid System for Compact Class Vehicles," SAE Paper 2009-01-1332, 2009

[35] Jerome Meisel, "An Analytic Foundation for the Toyota Prius THS-II Powertrain with a Comparison to a Strong Parallel Hybrid-Electric Powertrain," SAE Paper 2006-01-0666, 2006 
[36] Jerome Meisel, "An Analytic Foundation for the Two-Mode Hybrid Electric Powertrain with a Comparison to the Single-Mode Toyota Prius THS-II Powrtrain," SAE Paper 200901-1321, 2009

[37] Sungtae Cho, Kukhyun Ahn and Jang Moo Lee, "Efficiency of the Planetary Gear Hybrid Powertrain," Proc. IMech Part D: J. Automobile Engineering, Vol. 220, pp 1445 - 1454, 2006

[38] Kukhyun Ahn, et al., "Engine Operation for the Planetary Gear Hybrid Powertrain," Proc. IMech Part D: J. Automobile Engineering, Vol. 220, pp 1727 - 1735, 2006

[39] Michael Duoba and Robert Larsen, "HEV Dynamometer Testing with State-of-Charge Corrections in the 1995 HEV Challenge," SAE Paper 960740, 1996

[40] SAE Recommended Practice, "Recommended Practice for Measuring the Exhaust Emissions and Fuel Economy of Hybrid-Electric Vehicles," SAE J1711, March, 1999

[41] David L. McKain, et al., "Characterization of Emissions from Hybrid-Electric and Conventional Transit Buses,” SAE Paper 2000-01-2011, 2000

[42] Henning Lohse-Busch, et al., "A Modular Automotive Hybrid Testbed Designed to Evaluate Various Components in the Vehicle System," SAE Paper 2009-01-1315, 2009

[43] T. Gillespie, "Fundamentals of Vehicle Dynamics," SAE International, Warrendale, PA, March 1992

[44] J. Miller, Propulsion Systems for Hybrid Vehicles, The Institution of Electrical Engineers, 2004

[45] M. Ehsani, et al., Modern Electric, Hybrid Electric, and Fuel Cell Vehicles/Fundamentals, Theory, and Design, CRC Press, 2005

[46] G. Sovran and D. Blaser, "A Contribution to Understanding Automotive Fuel Economy and Its Limits,” SAE Paper 2003-01-2070, 2003

[47] E. Nam and R. Giannelli, "Fuel Consumption Modeling of Conventional and Advanced Technology Vehicles in the Physical Emission Rate Estimator (PERE)," EPA420-P-05-001, Feburary 2005

[48] Edward K. Nam, "Fuel Consumption Modeling of Hybrid Vehicles in PERE," SAE Paper 2005-01-0627, 2005 
[49] E. Nam and J. Sorab, "Friction Reduction Trends in Modern Engines," SAE Paper 2004-011456,2004

[50] Powertrain System Analysis Toolkit (C) (PSAT), http://www.transportation.anl.gov/modeling_simulation/PSAT/

[51] A. Rousseau, et al., "Integrating Data, Performing Quality Assurance, and Validating the Vehicle Model for the 2004 Prius Using PSAT," SAE Paper 2006-01-0667, 2006

[52] Annual Certification Test Results and Data from EPA, http://www.epa.gov/OMS/crttst.htm

[53] Advanced Powertrain Research Facility in Argonne National Laboratory, http://www.transportation.anl.gov/facilities/aprf.html

[54] Brett C. Singer, et al., "A Fuel-Based Approach to Estimating Motor Vehicle Cold-Start Emissions," Journal of the Air \& Waste Management Association, Vol. 49, pp 125 - 135, 1999

[55] Martha Christenson, et al., "The Effect of Driving Conditions and Ambient Temperature on Light Duty Gasoline-Electric Hybrid Vehicles (1): Particulate Matter Emission Rates and Size Distributions," SAE Paper 2007-01-2136, 2007

[56] Martha Christenson, et al., "The Effect of Driving Conditions and Ambient Temperature on Light Duty Gasoline-Electric Hybrid Vehicles (2): Fuel Consumption and Gaseous Pollutant Emission Rates," SAE Paper 2007-01-2137, 2007

[57] Hamilton H. Mabie and Charles F. Reinholtz, Mechanisms and Dynamics of Machinery, Wiley, 1987 


\section{Appendix A Speed, Torque and Power of a Planetary Gear Set}

The speed of a planetary gear set is very straightforward to understand because the speed of one gear can be easily obtained from one constraint equation if the speeds of other two gears are given. However, the power and torque of a planetary gear set is a little more complicated. The following sections explains basic equations and understanding of a planetary gear set which is described in some text books [34][57] and literatures [29][30][36].

\section{A.1. Speed}

The speed of a planetary gear set can be determined by equation (A.1) below.

$$
(1+n) \omega_{c}-n \omega_{s}-\omega_{r}=0
$$

Where: $n$ : Planetary gear ratio (number of teeth in ring gear to number of teeth in sun gear, 0.3846 for Toyota Prius)

$\omega_{c}$ : Angular velocity of carrier $(\mathrm{rad} / \mathrm{s})$

$\omega_{s}:$ Angular velocity of sun gear $(\mathrm{rad} / \mathrm{s})$

$\omega_{r}$ : Angular velocity of ring gear $(\mathrm{rad} / \mathrm{s})$

In this equation, two angular velocities should be given for calculating the angular velocity of the other component. For example, if the speeds of the engine and the MG2 speed are given, we can determine the MG1 speed. In Toyota Prius, the carrier speed is the engine speed, the sun gear speed is the MG1 speed, and the ring gear speed is the MG2 speed respectively. Note that their 
speed is limited by its own control strategy as previously mentioned; MG1: $\pm 10,000 \mathrm{rpm}, \mathrm{MG} 2$ : $0 \sim 6,500 \mathrm{rpm}$, and engine: $0 \sim 5,000 \mathrm{rpm}$ respectively [10].

\section{A.2. Torque}

Torque at each component in planetary gear set can be calculated using equations (A.2) and (A.3) below.

$$
\begin{aligned}
& T_{r}=\frac{1}{1+n} T_{e} \\
& T_{s}=\frac{n}{1+n} T_{e}
\end{aligned}
$$

Where: $T_{r}$ : Torque transmitted to ring gear $(\mathrm{Nm})$

$T_{e}$ : Engine torque $(\mathrm{Nm})$

$T_{s}$ : Torque transmitted to sun gear $(\mathrm{Nm})$

Note that $T_{r}$ in equation (A.2) does not include MG2 torque because the ring gear is the output shaft of planetary gear set as shown in Figure 1, but additional motor, MG2, is connected as well. Therefore, the overall torque/power of the PSD should be considered at the output shaft which is directly connected to the ring gear. The output shaft speed is the ring gear speed. More details are explained in later sections.

\section{A.3 Power}

The power of the gears in planetary gear set can be easily calculated by multiplying angular velocity by torque as listed in the following equations.

$$
P_{c}=T_{c} \omega_{c}, P_{r}=T_{r} \omega_{r}, P_{s}=T_{s} \omega_{s}
$$

Where: $P_{c}$ : Power transmitted to carrier from engine $\left(P_{c}=P_{e}\right)(\mathrm{W})$

$T_{c}$ : Torque transmitted to carrier from engine $\left(T_{c}=T_{e}\right)(\mathrm{W})$

$P_{r}$ : Power transmitted to ring gear (W)

$P_{s}:$ Power transmitted to sun gear $(\mathrm{W})$

The power transmitted to the ring gear can be calculated from equation (5) as well.

$$
P_{r}=P_{c}-P_{s}=P_{e}-P_{s}
$$


Now, all power/torque of the planetary gear set are known, the output power of transmission can be obtained from equation (A.6) below.

$$
P_{\text {trans, out }}=P_{r}+P_{M G 2}
$$

Note that all variables in power discussed here is based on output, so positive numbers represent that the power is coming out of the component or system and negative numbers represent that the power is going into the component or system respectively. 


\section{Appendix B Result Data from Simulations and Vehicle Testing}

Table B.1 Results from the simple vehicle model for finding optimized fuel economy of PnG driving (2007 Ford Focus)

\begin{tabular}{|r|r||r|r||r|r|}
\hline \multicolumn{2}{|c||}{$20-30 \mathrm{mph}$} & \multicolumn{2}{c||}{$30-40 \mathrm{mph}$} & \multicolumn{2}{c|}{$40-50 \mathrm{mph}$} \\
\cline { 1 - 2 } $\begin{array}{c}\text { Acceleration } \\
\text { Time (sec) }\end{array}$ & $\begin{array}{c}\text { Fuel Economy } \\
(\mathrm{mpg})\end{array}$ & $\begin{array}{c}\text { Acceleration } \\
\text { Time (sec) }\end{array}$ & $\begin{array}{c}\text { Fuel Economy } \\
(\mathrm{mpg})\end{array}$ & $\begin{array}{c}\text { Acceleration } \\
\text { Time (sec) }\end{array}$ & $\begin{array}{c}\text { Fuel Economy } \\
(\mathrm{mpg})\end{array}$ \\
\hline 4.2 & 146.9 & 6.1 & 112.7 & 9.4 & 85.3 \\
\hline 4.2 & 146.8 & 6.4 & 111.6 & 10.0 & 84.5 \\
\hline 4.6 & 144.0 & 7.1 & 109.5 & 11.4 & 82.9 \\
\hline 5.1 & 140.9 & 8.0 & 107.1 & 13.1 & 81.1 \\
\hline 5.8 & 137.2 & 9.1 & 104.3 & 15.4 & 78.9 \\
\hline 6.6 & 132.8 & 10.6 & 101.0 & 18.7 & 76.4 \\
\hline 7.7 & 127.7 & 12.7 & 97.1 & 24.0 & 73.4 \\
\hline 9.3 & 121.3 & 15.8 & 92.3 & 33.2 & 69.6 \\
\hline 11.6 & 113.5 & 21.0 & 86.3 & 54.4 & 64.8 \\
\hline 15.5 & 103.5 & 31.1 & 78.5 & N/A & N/A \\
\hline 23.4 & 90.2 & 60.4 & 68.1 & N/A & N/A \\
\hline 47.7 & 71.6 & N/A & N/A & N/A & N/A \\
\hline
\end{tabular}


Table B.2 Results from the simple vehicle model for PnG in $20-30 \mathrm{mph}$ and steady speed at 25 mph (2007 Ford Focus)

\begin{tabular}{|c|c|c|c|c|c|c|}
\hline \multirow{2}{*}{$\begin{array}{c}\text { Acceleration } \\
\text { Time } \\
(\mathrm{sec})\end{array}$} & \multirow{2}{*}{$\begin{array}{l}\text { Cycle } \\
\text { Time } \\
(\mathrm{sec}) \\
\end{array}$} & \multirow{2}{*}{$\begin{array}{l}\text { Distance } \\
\text { (mile) }\end{array}$} & \multicolumn{2}{|c|}{ Fuel Economy (mpg) } & \multirow{2}{*}{\multicolumn{2}{|c|}{$\begin{array}{c}\text { Average Speed for } \\
\text { Steady Speed } \\
(\mathrm{mph})\end{array}$}} \\
\hline & & & PnG & $\begin{array}{r}\text { Steady } \\
\text { Speed }\end{array}$ & & \\
\hline 5.0 & 50.0 & 0.345 & 140.5 & 46.0 & & .82 \\
\hline 10.0 & 55.0 & 0.379 & 118.2 & 46.0 & & .84 \\
\hline 15.0 & 60.0 & 0.414 & 104.3 & 46.0 & & 86 \\
\hline 20.0 & 65.0 & 0.449 & 94.8 & 46.0 & & .88 \\
\hline 25.1 & 70.1 & 0.485 & 88.0 & 46.0 & & 91 \\
\hline 30.0 & 75.0 & 0.519 & 82.9 & 46.0 & & 93 \\
\hline \multirow{2}{*}{$\begin{array}{l}\text { Acceleration } \\
\text { Time } \\
(\mathrm{sec})\end{array}$} & \multicolumn{2}{|c|}{$\begin{array}{c}\text { Engine Speed } \\
(\mathrm{rpm})\end{array}$} & \multicolumn{2}{|c|}{$\begin{array}{c}\text { Constant Engine } \\
\text { Torque }(\mathrm{Nm})\end{array}$} & \multicolumn{2}{|c|}{$\begin{array}{l}\text { Engine Overall } \\
\text { Efficiency (\%) }\end{array}$} \\
\hline & PnG & \begin{tabular}{|r|}
$\begin{array}{r}\text { Steady } \\
\text { Speed }\end{array}$ \\
\end{tabular} & PnG & $\begin{array}{l}\text { Steady } \\
\text { Speed } \\
\end{array}$ & PnG & $\begin{array}{l}\text { Steady } \\
\text { Speed } \\
\end{array}$ \\
\hline 5.0 & \multirow{6}{*}{$\begin{array}{c}1341 \\
\sim 2011\end{array}$} & 1664 & 102.3 & 10.2 & 30.7 & \multirow{6}{*}{9.9} \\
\hline 10.0 & & 1665 & 56.3 & 10.2 & 25.8 & \\
\hline 15.0 & & 1667 & 41.0 & 10.2 & 22.7 & \\
\hline 20.0 & & 1668 & 33.3 & 10.2 & 20.7 & \\
\hline 25.1 & & 1670 & 28.7 & 10.2 & 19.2 & \\
\hline 30.0 & & 1671 & 25.7 & 10.2 & 18.1 & \\
\hline
\end{tabular}

Table B.3 Results from the simple vehicle model for PnG in $30-40 \mathrm{mph}$ and steady speed at 35 mph (2007 Ford Focus)

\begin{tabular}{|c|c|c|c|c|c|c|}
\hline \multirow{2}{*}{$\begin{array}{l}\text { Acceleration } \\
\text { Time } \\
(\mathrm{sec})\end{array}$} & \multirow{2}{*}{$\begin{array}{l}\text { Cycle } \\
\text { Time } \\
(\mathrm{sec}) \\
\end{array}$} & \multirow{2}{*}{$\begin{array}{l}\text { Distance } \\
\text { (mile) }\end{array}$} & \multicolumn{2}{|c|}{ Fuel Economy (mpg) } & \multirow{2}{*}{\multicolumn{2}{|c|}{$\begin{array}{l}\text { Average Speed fo } \\
\text { Steady Speed } \\
\text { (mph) }\end{array}$}} \\
\hline & & & PnG & $\begin{array}{l}\text { Steady } \\
\text { Speed }\end{array}$ & & \\
\hline 10.0 & 44.2 & 0.428 & 102.1 & 53.5 & & .83 \\
\hline 15.0 & 49.2 & 0.477 & 93.3 & 53.5 & & .87 \\
\hline 20.0 & 54.3 & 0.526 & 87.1 & 53.4 & & .91 \\
\hline 25.0 & 59.2 & 0.575 & 82.6 & 53.4 & & .94 \\
\hline 29.9 & 64.2 & 0.624 & 79.2 & 53.4 & & .97 \\
\hline \multirow{2}{*}{$\begin{array}{c}\text { Acceleration } \\
\text { Time } \\
(\mathrm{sec})\end{array}$} & \multicolumn{2}{|c|}{$\begin{array}{c}\text { Engine Speed } \\
(\mathrm{rpm})\end{array}$} & \multicolumn{2}{|c|}{$\begin{array}{c}\text { Constant Engine } \\
\text { Torque }(\mathrm{Nm})\end{array}$} & \multicolumn{2}{|c|}{$\begin{array}{l}\text { Engine Overall } \\
\text { Efficiency }(\%)\end{array}$} \\
\hline & PnG & \begin{tabular}{|l} 
Steady \\
Speed
\end{tabular} & PnG & $\begin{array}{l}\text { Steady } \\
\text { Speed }\end{array}$ & PnG & $\begin{array}{l}\text { Steady } \\
\text { Speed }\end{array}$ \\
\hline 10.0 & \multirow{5}{*}{$1429 \sim 1905$} & 1659 & 83.9 & 18.9 & 29.2 & \multirow{5}{*}{15.1} \\
\hline 15.0 & & 1661 & 62.3 & 18.9 & 26.7 & \\
\hline 20.0 & & 1662 & 51.5 & 18.9 & 24.9 & \\
\hline 25.0 & & 1664 & 45.1 & 19.0 & 23.7 & \\
\hline 29.9 & & 1665 & 40.8 & 19.0 & 22.7 & \\
\hline
\end{tabular}


Table B.4 Results from the simple vehicle model for PnG in 40 - $50 \mathrm{mph}$ and steady speed sped at $45 \mathrm{mph}$ (2007 Ford Focus)

\begin{tabular}{|c|c|c|c|c|c|c|}
\hline \multirow{2}{*}{$\begin{array}{l}\text { Acceleration } \\
\text { Time } \\
\text { (sec) }\end{array}$} & \multirow{2}{*}{$\begin{array}{l}\text { Cycle } \\
\text { Time } \\
\text { (sec) }\end{array}$} & \multirow[b]{2}{*}{$\begin{array}{l}\text { Distance } \\
\text { (mile) }\end{array}$} & \multicolumn{2}{|c|}{ Fuel Economy (mpg) } & \multirow{2}{*}{\multicolumn{2}{|c|}{$\begin{array}{l}\text { Average Speed for } \\
\text { Steady Speed } \\
(\mathrm{mph})\end{array}$}} \\
\hline & & & $\mathrm{PnG}$ & $\begin{array}{l}\text { Steady } \\
\text { Speed }\end{array}$ & & \\
\hline 10.0 & 35.9 & 0.448 & 84.4 & 55.5 & & 4.9 \\
\hline 15.0 & 40.9 & 0.511 & 79.2 & 55.4 & & 5.0 \\
\hline 20.0 & 46.0 & 0.574 & 75.5 & 55.4 & & 5.0 \\
\hline 25.0 & 50.9 & 0.637 & 72.8 & 55.4 & & 5.1 \\
\hline 30.0 & 55.9 & 0.699 & 70.7 & 55.3 & & 5.1 \\
\hline \multirow{2}{*}{$\begin{array}{l}\text { Acceleration } \\
\text { Time } \\
(\mathrm{sec})\end{array}$} & \multicolumn{2}{|c|}{$\begin{array}{c}\text { Engine Speed } \\
(\mathrm{rpm})\end{array}$} & \multicolumn{2}{|c|}{$\begin{array}{c}\text { Constant Engine } \\
\text { Torque }(\mathrm{Nm})\end{array}$} & \multicolumn{2}{|c|}{$\begin{array}{l}\text { Engine Overall } \\
\text { Efficiency }(\%)\end{array}$} \\
\hline & PnG & \begin{tabular}{|r} 
Steady \\
Speed \\
\end{tabular} & $\mathrm{PnG}$ & $\begin{array}{l}\text { Steady } \\
\text { Speed } \\
\end{array}$ & $\mathrm{PnG}$ & $\begin{array}{l}\text { Steady } \\
\text { Speed } \\
\end{array}$ \\
\hline 10.0 & \multirow{5}{*}{$1424 \sim 1780$} & 1597 & 120.4 & 33.4 & 31.8 & \multirow{5}{*}{20.7} \\
\hline 15.0 & & 1599 & 91.5 & 33.5 & 29.8 & \\
\hline 20.0 & & 1600 & 77.1 & 33.5 & 28.5 & \\
\hline 25.0 & & 1602 & 68.5 & 33.5 & 27.5 & \\
\hline 30.0 & & 1603 & 62.8 & 33.6 & 26.7 & \\
\hline
\end{tabular}

Table B.5 Results from PSAT for PnG in 20 - $30 \mathrm{mph}$ and steady speed at $25 \mathrm{mph}$ (2007 Ford Focus)

\begin{tabular}{|c|c|c|c|c|c|}
\hline \multirow{2}{*}{$\begin{array}{c}\text { Acceleration } \\
\text { Time } \\
(\mathrm{sec})\end{array}$} & \multirow{2}{*}{$\begin{array}{l}\text { Cycle } \\
\text { Time } \\
\text { (sec) }\end{array}$} & \multirow{2}{*}{$\begin{array}{c}\text { Distance } \\
\text { (mile) }\end{array}$} & \multicolumn{3}{|c|}{ Fuel Economy (mpg) } \\
\hline & & & & PnG & $\begin{array}{l}\text { Steady } \\
\text { Speed }\end{array}$ \\
\hline 5.0 & 34.3 & 0.228 & & 94.5 & \multirow{6}{*}{$\begin{array}{c}45.1 \\
\text { (at } 25 \mathrm{mph})\end{array}$} \\
\hline 10.0 & 39.3 & 0.266 & & 86.7 & \\
\hline 15.0 & 44.3 & 0.303 & & 77.3 & \\
\hline 20.0 & 49.3 & 0.340 & & 69.9 & \\
\hline 25.0 & 54.3 & 0.376 & & 65.4 & \\
\hline 30.0 & 59.3 & 0.413 & & 62.7 & \\
\hline \multirow{2}{*}{$\begin{array}{l}\text { Acceleration } \\
\text { Time } \\
(\mathrm{sec})\end{array}$} & \multicolumn{2}{|c|}{$\begin{array}{l}\text { Engine Speed } \\
\text { (rpm) }\end{array}$} & \multicolumn{2}{|c|}{$\begin{array}{l}\text { Average Engine } \\
\text { Efficiency }(\%)\end{array}$} & \multirow{2}{*}{$\begin{array}{l}\text { Average Speed } \\
\text { of PnG (mph) }\end{array}$} \\
\hline & PnG & $\begin{array}{l}\text { Steady } \\
\text { Speed }\end{array}$ & PnG & $\begin{array}{r}\text { Steady } \\
\text { Speed } \\
\end{array}$ & \\
\hline 5.0 & 1605 2348 & \multirow{6}{*}{2006} & 30.5 & \multirow{6}{*}{16.7} & 24.0 \\
\hline 10.0 & $1605 \sim 2380$ & & 29.6 & & 24.4 \\
\hline 15.0 & $1605 \sim 2390$ & & 27.1 & & 24.6 \\
\hline 20.0 & $1605 \sim 2395$ & & 24.9 & & 24.8 \\
\hline 25.0 & $1605 \sim 2399$ & & 23.6 & & 24.9 \\
\hline 30.0 & $1605 \sim 2400$ & & 22.7 & & 25.0 \\
\hline
\end{tabular}


Table B.6 Results from PSAT for PnG in 30 - $40 \mathrm{mph}$ and steady speed at $35 \mathrm{mph}$ (2007 Ford Focus)

\begin{tabular}{|c|c|c|c|c|c|}
\hline \multirow{2}{*}{$\begin{array}{c}\text { Acceleration } \\
\text { Time } \\
\text { (sec) }\end{array}$} & \multirow{2}{*}{$\begin{array}{l}\text { Cycle } \\
\text { Time } \\
(\mathrm{sec})\end{array}$} & \multirow{2}{*}{$\begin{array}{l}\text { Distance } \\
\text { (mile) }\end{array}$} & \multicolumn{3}{|c|}{ Fuel Economy (mpg) } \\
\hline & & & & PnG & $\begin{array}{l}\text { Steady } \\
\text { Speed }\end{array}$ \\
\hline 10.0 & 32.5 & 0.312 & & 75.1 & \multirow{5}{*}{$\begin{array}{c}49.0 \\
\text { (at } 35 \mathrm{mph} \text { ) }\end{array}$} \\
\hline 15.0 & 37.5 & 0.362 & & 71.3 & \\
\hline 20.0 & 42.5 & 0.412 & & 69 & \\
\hline 25.0 & 47.5 & 0.462 & & 66.4 & \\
\hline 30.0 & 52.5 & 0.513 & & 63.8 & \\
\hline \multirow{2}{*}{$\begin{array}{c}\text { Acceleration } \\
\text { Time } \\
(\mathrm{sec})\end{array}$} & \multicolumn{2}{|c|}{$\begin{array}{l}\text { Engine Speed } \\
(\mathrm{rpm})\end{array}$} & \multicolumn{2}{|c|}{$\begin{array}{l}\text { Average Engine } \\
\text { Efficiency }(\%)\end{array}$} & \multirow{2}{*}{$\begin{array}{l}\text { Average Speed } \\
\text { of PnG (mph) }\end{array}$} \\
\hline & $\mathrm{PnG}$ & $\begin{array}{l}\text { Steady } \\
\text { Speed }\end{array}$ & PnG & $\begin{array}{l}\text { Steady } \\
\text { Speed }\end{array}$ & \\
\hline 10.0 & 1711 2262 & \multirow{5}{*}{1997} & 31.8 & \multirow{5}{*}{22.0} & 34.5 \\
\hline 15.0 & $1711 \sim 2269$ & & 30.8 & & 34.7 \\
\hline 20.0 & $1711 \sim 2273$ & & 30.2 & & 34.9 \\
\hline 25.0 & $1711 \sim 2275$ & & 29.3 & & 35.0 \\
\hline 30.0 & $1711 \sim 2277$ & & 28.3 & & 35.2 \\
\hline
\end{tabular}

Table B.7 Results from PSAT for PnG in 40 - $50 \mathrm{mph}$ and steady speed at $45 \mathrm{mph}$ (2007 Ford Focus)

\begin{tabular}{|c|c|c|c|c|c|}
\hline \multirow{2}{*}{$\begin{array}{c}\text { Acceleration } \\
\text { Time } \\
(\mathrm{sec})\end{array}$} & \multirow{2}{*}{$\begin{array}{l}\text { Cycle } \\
\text { Time } \\
(\mathrm{sec})\end{array}$} & \multirow{2}{*}{$\begin{array}{c}\text { Distance } \\
\text { (mile) }\end{array}$} & \multicolumn{3}{|c|}{ Fuel Economy (mpg) } \\
\hline & & & & PnG & $\begin{array}{l}\text { Steady } \\
\text { Speed }\end{array}$ \\
\hline 10.0 & 27.7 & 0.343 & & 59.4 & \multirow{5}{*}{$\begin{array}{c}50.2 \\
\text { (at } 45 \mathrm{mph} \text { ) }\end{array}$} \\
\hline 15.0 & 32.7 & 0.407 & & 59.8 & \\
\hline 20.0 & 37.7 & 0.471 & & 59.6 & \\
\hline 25.0 & 42.7 & 0.535 & & 58.3 & \\
\hline 30.0 & 47.7 & 0.600 & & 57.3 & \\
\hline \multirow{2}{*}{$\begin{array}{l}\text { Acceleration } \\
\text { Time } \\
(\mathrm{sec})\end{array}$} & \multicolumn{2}{|c|}{$\begin{array}{l}\text { Engine Speed } \\
(\text { rpm })\end{array}$} & \multicolumn{2}{|c|}{$\begin{array}{l}\text { Average Engine } \\
\text { Efficiency }(\%)\end{array}$} & \multirow{2}{*}{$\begin{array}{c}\text { Average Speed } \\
\text { of PnG (mph) }\end{array}$} \\
\hline & PnG & $\begin{array}{l}\text { Steady } \\
\text { Speed }\end{array}$ & PnG & $\begin{array}{r}\text { Steady } \\
\text { Speed }\end{array}$ & \\
\hline 10.0 & $1706 \sim 2118$ & \multirow{5}{*}{1997} & 31.1 & \multirow{5}{*}{27.3} & 44.6 \\
\hline 15.0 & $1706 \sim 2123$ & & 31.8 & & 44.8 \\
\hline 20.0 & $1706 \sim 2126$ & & 32.0 & & 45.0 \\
\hline 25.0 & $1706 \sim 2128$ & & 31.5 & & 45.1 \\
\hline 30.0 & $1706 \sim 2129$ & & 31.1 & & 45.3 \\
\hline
\end{tabular}


Table B.8 Results from PSAT for PnG in 20 - $30 \mathrm{mph}$ and steady speed at $25 \mathrm{mph}$ (2004 Toyota Prius)

\begin{tabular}{|c|c|c|c|c|c|}
\hline \multirow{2}{*}{$\begin{array}{l}\text { Acceleration } \\
\text { Time } \\
(\mathrm{sec})\end{array}$} & \multirow{2}{*}{$\begin{array}{l}\text { Cycle } \\
\text { Time } \\
(\mathrm{sec})\end{array}$} & \multirow{2}{*}{$\begin{array}{l}\text { Distance } \\
\text { (mile) }\end{array}$} & \multicolumn{3}{|c|}{ Fuel Economy (mpg) } \\
\hline & & & \multicolumn{2}{|r|}{ PnG } & $\begin{array}{l}\text { Steady } \\
\text { Speed }\end{array}$ \\
\hline 5.0 & 2129.6 & 14.70 & \multicolumn{2}{|r|}{113.7} & \multirow{6}{*}{$\begin{array}{c}82.1 \\
\text { (at } 25 \mathrm{mph} \text { ) }\end{array}$} \\
\hline 10.0 & 2005.2 & 13.87 & & 133.3 & \\
\hline 15.0 & 2003.1 & 13.90 & & 132.1 & \\
\hline 20.0 & 1971.0 & 13.70 & & 124.4 & \\
\hline 25.0 & 2194.8 & 15.30 & & 120.2 & \\
\hline 30.0 & 1892.7 & 13.23 & & 99.1 & \\
\hline \multirow{2}{*}{$\begin{array}{l}\text { Acceleration } \\
\text { Time } \\
(\mathrm{sec})\end{array}$} & \multirow{2}{*}{$\begin{array}{l}\text { Efficiency of } \\
\text { KE storage (\%) }\end{array}$} & \multicolumn{3}{|c|}{$\begin{array}{l}\text { Average Engine } \\
\text { Efficiency }(\%)\end{array}$} & \multirow{2}{*}{$\begin{array}{c}\text { Average Speed } \\
\text { of PnG (mph) }\end{array}$} \\
\hline & & \multicolumn{2}{|c|}{ PnG } & $\begin{array}{r}\text { Steady } \\
\text { Speed } \\
\end{array}$ & \\
\hline \multirow{3}{*}{5.0} & \multirow{3}{*}{31.9} & Accel & 35.4 & & \multirow{3}{*}{24.8} \\
\hline & & Coast & 17.9 & & \\
\hline & & Overall & 25.6 & & \\
\hline \multirow{3}{*}{10.0} & \multirow{3}{*}{32.4} & Accel & 34.9 & & \multirow{3}{*}{24.9} \\
\hline & & Coast & 23.1 & & \\
\hline & & Overall & 34.2 & & \\
\hline \multirow{3}{*}{15.0} & \multirow{3}{*}{34.4} & Accel & 34.4 & & \multirow{3}{*}{25.0} \\
\hline & & Coast & 21.0 & & \\
\hline & & Overall & 33.9 & 209 & \\
\hline \multirow{3}{*}{20.0} & \multirow{3}{*}{33.3} & Accel & 33.6 & 20.5 & \multirow{3}{*}{25.0} \\
\hline & & Coast & 20.3 & & \\
\hline & & Overall & 33.1 & & \\
\hline \multirow{3}{*}{25.0} & \multirow{3}{*}{33.1} & Accel & 33.0 & & \multirow{3}{*}{25.1} \\
\hline & & Coast & 19.3 & & \\
\hline & & Overall & 32.5 & & \\
\hline \multirow{3}{*}{30.0} & \multirow{3}{*}{41.4} & Accel & 32.4 & & \multirow{3}{*}{25.2} \\
\hline & & Coast & 13.8 & & \\
\hline & & Overall & 25.2 & & \\
\hline
\end{tabular}


Table B.9 Results from PSAT for PnG in 30 - $40 \mathrm{mph}$ and steady speed at $35 \mathrm{mph}$ (2004 Toyota Prius)

\begin{tabular}{|c|c|c|c|c|c|}
\hline \multirow{2}{*}{$\begin{array}{c}\text { Acceleration } \\
\text { Time } \\
(\mathrm{sec})\end{array}$} & \multirow{2}{*}{$\begin{array}{l}\text { Cycle } \\
\text { Time } \\
(\mathrm{sec})\end{array}$} & \multirow{2}{*}{$\begin{array}{l}\text { Distance } \\
\text { (mile) }\end{array}$} & \multicolumn{3}{|c|}{ Fuel Economy (mpg) } \\
\hline & & & \multicolumn{2}{|r|}{$\mathrm{PnG}$} & $\begin{array}{l}\text { Steady } \\
\text { Speed }\end{array}$ \\
\hline 5.0 & 1925.5 & 18.63 & & 92.1 & \multirow{6}{*}{$\begin{array}{c}76.1 \\
\text { (at } 35 \mathrm{mph} \text { ) }\end{array}$} \\
\hline 10.0 & 1949.2 & 18.90 & & 109.4 & \\
\hline 15.0 & 1972.0 & 19.16 & & 107.3 & \\
\hline 20.0 & 1954.8 & 19.03 & & 103.0 & \\
\hline 25.0 & 1956.9 & 19.08 & & 100.0 & \\
\hline 30.0 & 1993.3 & 19.48 & & 97.9 & \\
\hline \multirow{2}{*}{$\begin{array}{l}\text { Acceleration } \\
\text { Time } \\
(\mathrm{sec})\end{array}$} & \multirow{2}{*}{$\begin{array}{l}\text { Efficiency of } \\
\text { KE storage (\%) }\end{array}$} & \multicolumn{3}{|c|}{$\begin{array}{l}\text { Average Engine } \\
\text { Efficiency (\%) }\end{array}$} & \multirow{2}{*}{$\begin{array}{l}\text { Average Speed } \\
\text { of PnG (mph) }\end{array}$} \\
\hline & & \multicolumn{2}{|c|}{ PnG } & $\begin{array}{r}\text { Steady } \\
\text { Speed } \\
\end{array}$ & \\
\hline \multirow{3}{*}{5.0} & \multirow{3}{*}{33.8} & Accel & 35.7 & & \multirow{3}{*}{34.8} \\
\hline & & Coast & 18.7 & & \\
\hline & & Overall & 27.4 & & \\
\hline \multirow{3}{*}{10.0} & \multirow{3}{*}{34.4} & Accel & 35.7 & & \multirow{3}{*}{34.9} \\
\hline & & Coast & 25.5 & & \\
\hline & & Overall & 35.2 & & \\
\hline \multirow{3}{*}{15.0} & \multirow{3}{*}{35.5} & Accel & 35.1 & & \multirow{3}{*}{35.0} \\
\hline & & Coast & 25.5 & & \\
\hline & & Overall & 34.7 & 285 & \\
\hline \multirow{3}{*}{20.0} & \multirow{3}{*}{34.9} & Accel & 34.9 & & \multirow{3}{*}{35.0} \\
\hline & & Coast & 24.6 & & \\
\hline & & Overall & 34.6 & & \\
\hline \multirow{3}{*}{25.0} & \multirow{3}{*}{34.6} & Accel & 34.7 & & \multirow{3}{*}{35.1} \\
\hline & & Coast & 25.0 & & \\
\hline & & Overall & 34.5 & & \\
\hline \multirow{3}{*}{30.0} & \multirow{3}{*}{34.6} & Accel & 34.6 & & \multirow{3}{*}{35.2} \\
\hline & & Coast & 24.4 & & \\
\hline & & Overall & 34.4 & & \\
\hline
\end{tabular}


Table B.10 Results from PSAT for PnG in 40 - $50 \mathrm{mph}$ and steady speed at $45 \mathrm{mph}$ (2004 Toyota Prius)

\begin{tabular}{|c|c|c|c|c|c|}
\hline \multirow{2}{*}{$\begin{array}{l}\text { Acceleration } \\
\text { Time } \\
(\mathrm{sec})\end{array}$} & \multirow{2}{*}{$\begin{array}{l}\text { Cycle } \\
\text { Time } \\
(\mathrm{sec}) \\
\end{array}$} & \multirow{2}{*}{$\begin{array}{l}\text { Distance } \\
\text { (mile) }\end{array}$} & \multicolumn{3}{|c|}{ Fuel Economy (mpg) } \\
\hline & & & \multicolumn{2}{|r|}{ PnG } & $\begin{array}{r}\text { Steady } \\
\text { Speed }\end{array}$ \\
\hline 5.0 & 1977.6 & 24.63 & & 68.2 & \multirow{6}{*}{$\begin{array}{c}65.4 \\
\text { (at } 45 \mathrm{mph} \text { ) }\end{array}$} \\
\hline 10.0 & 2010.4 & 25.10 & & 87.2 & \\
\hline 15.0 & 2045.5 & 25.58 & & 86.0 & \\
\hline 20.0 & 2066.0 & 25.88 & & 83.0 & \\
\hline 25.0 & 1883.3 & 23.63 & & 80.7 & \\
\hline 30.0 & 2124.1 & 26.70 & & 78.2 & \\
\hline \multirow{2}{*}{$\begin{array}{c}\text { Acceleration } \\
\text { Time } \\
(\mathrm{sec})\end{array}$} & \multirow{2}{*}{$\begin{array}{l}\text { Efficiency of } \\
\text { KE storage (\%) }\end{array}$} & \multicolumn{3}{|c|}{$\begin{array}{l}\text { Average Engine } \\
\text { Efficiency (\%) }\end{array}$} & \multirow{2}{*}{$\begin{array}{l}\text { Average Speed } \\
\text { of PnG (mph) }\end{array}$} \\
\hline & & \multicolumn{2}{|c|}{ PnG } & $\begin{array}{r}\text { Steady } \\
\text { Speed } \\
\end{array}$ & \\
\hline \multirow{3}{*}{5.0} & \multirow{3}{*}{34.8} & Accel & 35.7 & & \multirow{3}{*}{44.8} \\
\hline & & Coast & 27.6 & & \\
\hline & & Overall & 32.3 & & \\
\hline \multirow{3}{*}{10.0} & \multirow{3}{*}{36.3} & Accel & 36.5 & & \multirow{3}{*}{44.9} \\
\hline & & Coast & 29.1 & & \\
\hline & & Overall & 36.0 & & \\
\hline \multirow{3}{*}{15.0} & \multirow{3}{*}{37.6} & Accel & 35.8 & & \multirow{3}{*}{45.0} \\
\hline & & Coast & 28.7 & & \\
\hline & & Overall & 35.5 & 338 & \\
\hline \multirow{3}{*}{20.0} & \multirow{3}{*}{37.3} & Accel & 35.6 & 30.0 & \multirow{3}{*}{45.1} \\
\hline & & Coast & 28.8 & & \\
\hline & & Overall & 35.3 & & \\
\hline \multirow{3}{*}{25.0} & \multirow{3}{*}{36.5} & Accel & 35.3 & & \multirow{3}{*}{45.2} \\
\hline & & Coast & 28.5 & & \\
\hline & & Overall & 35.1 & & \\
\hline \multirow{3}{*}{30.0} & \multirow{3}{*}{39.7} & Accel & 35.2 & & \multirow{3}{*}{45.3} \\
\hline & & Coast & 28.3 & & \\
\hline & & Overall & 35.0 & & \\
\hline
\end{tabular}


Table B.11 Results from vehicle testing for PnG in $20-30 \mathrm{mph}$ and steady speed at $25 \mathrm{mph}$ (2004 Toyota Prius)

\begin{tabular}{|l|r|r|r|r|r|}
\hline & \multicolumn{1}{|c|}{ Test 1 } & \multicolumn{1}{c|}{ Test 4 } & \multicolumn{1}{c|}{ Test 7 } & \multicolumn{1}{c|}{ Test 10 } & \multicolumn{1}{c|}{ Test 13 } \\
\cline { 2 - 6 } & $\begin{array}{c}\text { SS } \\
\text { at 25 mph }\end{array}$ & $\begin{array}{c}\text { PnG: } \\
\text { True } \\
\text { Coasting }\end{array}$ & $\begin{array}{c}\text { PnG: engine } \\
\text { Compression } \\
\text { Braking } \\
\text { w/ Gear D }\end{array}$ & $\begin{array}{c}\text { PnG: Power } \\
\text { Decel w/ same } \\
\text { speed as } \\
\text { true coasting }\end{array}$ & $\begin{array}{c}\text { PnG: Power } \\
\text { Decel w/ 0.6 * } \\
\text { Decel of true } \\
\text { coasting }\end{array}$ \\
\hline Cycle Time (s) & 1713.1 & 1317.8 & 1060.5 & 1317.6 & 1751.9 \\
\hline Distance (mile) & 12.04 & 9.18 & 7.40 & 9.22 & 12.03 \\
\hline Avg. Speed (mph) & 25.3 & 25.1 & 25.1 & 25.2 & 24.7 \\
\hline Fuel Use (g) & 288.1 & 416.3 & 203.3 & 242.0 & 0.1028 \\
\hline Fuel Use (gallon) & 0.1027 & 0.1484 & 0.0725 & 0.0863 & 123.5 \\
\hline $\begin{array}{l}\text { SOC Corrected } \\
\text { Fuel Economy } \\
\text { (mpg) }\end{array}$ & 117.2 & 60.4 & 103.1 & 118.5 & \\
\hline
\end{tabular}

Table B.12 Results from vehicle testing for PnG in 30-40 mph and steady speed at $35 \mathrm{mph}$ (2004 Toyota Prius)

\begin{tabular}{|c|c|c|c|c|c|}
\hline & Test 2 & Test 5 & Test 8 & Test 11 & Test 14 \\
\hline & $\begin{array}{c}\mathrm{SS} \\
\text { at } 35 \mathrm{mph}\end{array}$ & $\begin{array}{c}\text { PnG: } \\
\text { True } \\
\text { Coasting }\end{array}$ & $\begin{array}{l}\text { PnG: engine } \\
\text { Compression } \\
\text { Braking } \\
\text { w/ Gear D }\end{array}$ & $\begin{array}{l}\text { PnG: Power } \\
\text { Decel w/ same } \\
\text { speed as } \\
\text { true coasting }\end{array}$ & $\begin{array}{l}\text { PnG: Power } \\
\text { Decel w/ } 0.6 * \\
\text { Decel of true } \\
\text { coasting }\end{array}$ \\
\hline Cycle Time (s) & 1544.3 & 553.8 & 1254.4 & 681.7 & 1495.3 \\
\hline Distance (mile) & 15.00 & 5.37 & 12.25 & 6.64 & 14.47 \\
\hline Avg. Speed (mph) & 35.0 & 34.9 & 35.2 & 35.1 & 34.9 \\
\hline Fuel Use $(\mathrm{g})$ & 455.6 & 228.5 & 467.6 & 221.4 & 412.7 \\
\hline Fuel Use (gallon) & 0.1624 & 0.0814 & 0.1667 & 0.0789 & 0.1471 \\
\hline $\begin{array}{l}\text { SOC Corrected } \\
\text { Fuel Economy } \\
(\mathrm{mpg})\end{array}$ & 92.4 & 61.6 & 91.3 & 102.4 & 101.0 \\
\hline
\end{tabular}

Table B.13 Results from vehicle testing for PnG in 40 - $50 \mathrm{mph}$ and steady speed at $45 \mathrm{mph}$ (2004 Toyota Prius)

\begin{tabular}{|c|c|c|c|c|c|}
\hline & Test 3 & Test 6 & Test 9 & Test 10 & Test 15 \\
\hline & $\begin{array}{c}\text { SS } \\
\text { at } 45 \mathrm{mph}\end{array}$ & $\begin{array}{c}\text { PnG: } \\
\text { True } \\
\text { Coasting }\end{array}$ & $\begin{array}{l}\text { PnG: engine } \\
\text { Compression } \\
\text { Braking } \\
\text { w/ Gear D }\end{array}$ & $\begin{array}{l}\text { PnG: Power } \\
\text { Decel w/ same } \\
\text { speed as } \\
\text { true coasting }\end{array}$ & $\begin{array}{l}\text { PnG: Power } \\
\text { Decel w/ } 0.6^{*} \\
\text { Decel of true } \\
\text { coasting }\end{array}$ \\
\hline Cycle Time (s) & 1118.7 & 1865.7 & 1101.7 & 1196.9 & 1290.3 \\
\hline Distance (mile) & 13.97 & 23.36 & 13.82 & 14.98 & 16.07 \\
\hline Avg. Speed (mph) & 45.0 & 45.1 & 45.2 & 45.1 & 44.8 \\
\hline Fuel Use (g) & 520.0 & 1136.7 & 584.0 & 558.8 & 622.8 \\
\hline Fuel Use (gallon) & 0.1854 & 0.4052 & 0.2082 & 0.1992 & 0.2220 \\
\hline $\begin{array}{l}\text { SOC Corrected } \\
\text { Fuel Economy } \\
\text { (mpg) }\end{array}$ & 75.4 & 57.5 & 73.2 & 74.2 & 75.6 \\
\hline
\end{tabular}




\section{Appendix C SOC Correction for Fuel Economy of HEVs Using Linear Regression}

HEVs can use and store energy in an additional storage, for example a battery, and it can alter fuel consumption over a drive cycle. For example, if the SOC of the battery is increased at the end of a drive cycle, it means that the battery is charged compared to the initial condition and the engine may consume extra fuel. Therefore, SAE recommends balancing the SOC of the battery for an HEV within $\pm 1 \%$ between initial and final conditions. However, it is very difficult to balance initial and final SOCs in real vehicle testing so many studies use SOC correction methods as introduced in section 2.5. The fuel consumption or the fuel economy of each drive cycle is plotted over the net electric energy or delta SOC respectively. After that, a linear regression line can be drawn trough the scattered data and the y-intercept can be either the SOC corrected fuel consumption or fuel economy. This basic idea is well illustrated in Figure 6.12.

Here, linear regression methods used in this study are briefly introduced and described. There are many linear regression methods, but two built-in methods in MATLABC are selected in the beginning; the ordinary least squares method and the robust regression method. Figure C.1 shows an example of SOC correction by the ordinary least squares (dashed green line) and robust regression (red line) methods. In this example, two regression lines are well matched for the scattered data points. However, the least squares fit for another example in Figure C.2 is skewed to two outliers, so the y-intercept value of the equation is quite different from that of the equation by the robust regression. It can possibly affect the fuel consumption results. In general, the least squares method is not robust to outliers. However, the robust regression fit in Figure C.2 is not 
affected by outliers. Therefore, the robust regression is used for estimating the SOC corrected fuel economy of the HEV testing in this study. Note that the robust regression uses iteratively reweighted least squares so it is very robust to outliers.

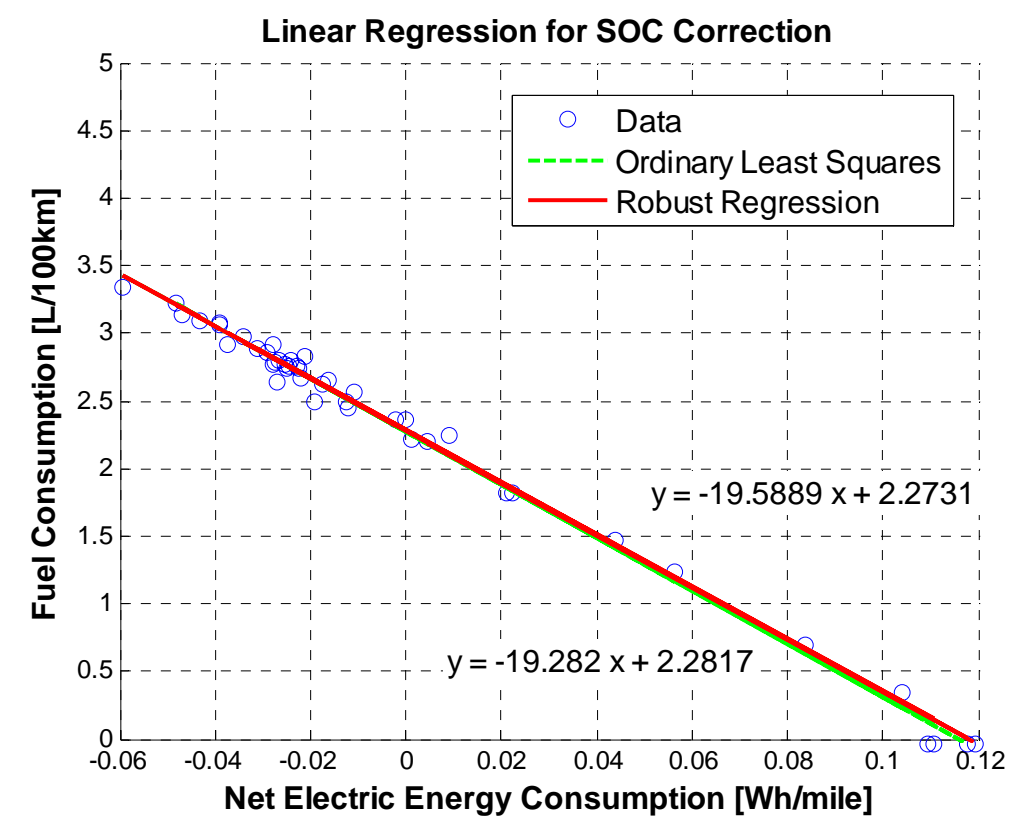

Figure C.1 Example of SOC correction using linear regression from test 7

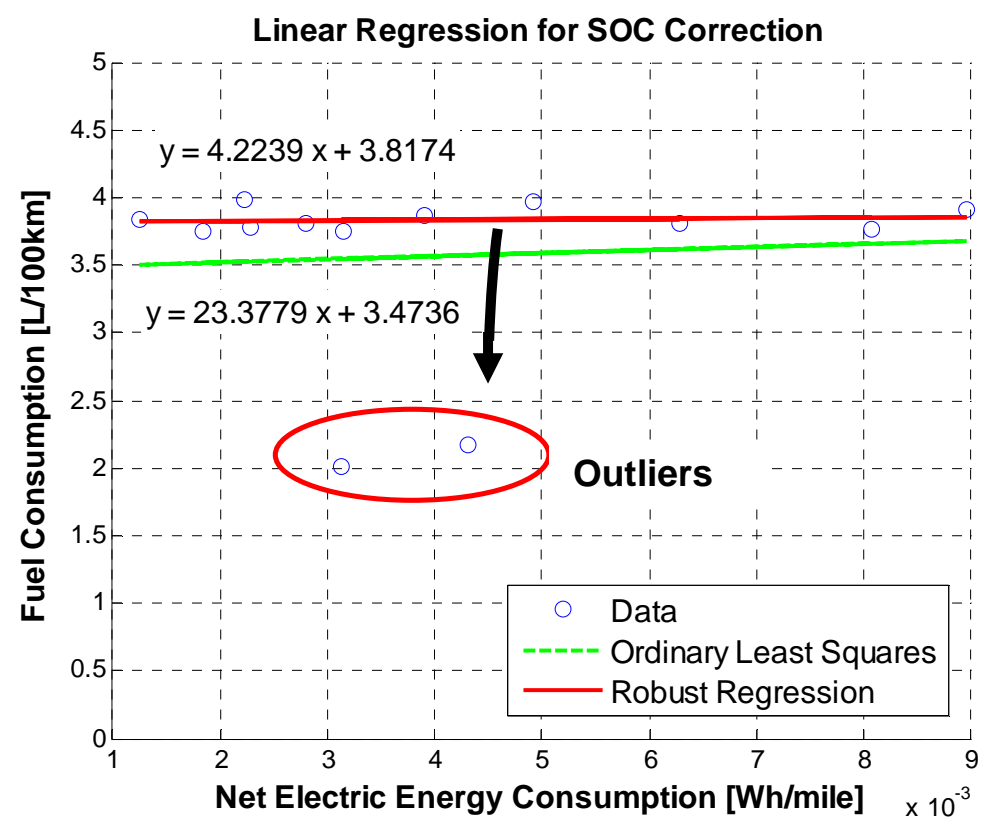

Figure C.2 Example of SOC correction using linear regression from test 5 